\title{
Autism Spectrum Disorders among Adults: Burden of Healthcare Services' Utilization and Expenditures
}

Rini Vohra

Follow this and additional works at: https://researchrepository.wvu.edu/etd

\section{Recommended Citation}

Vohra, Rini, "Autism Spectrum Disorders among Adults: Burden of Healthcare Services' Utilization and Expenditures" (2016). Graduate Theses, Dissertations, and Problem Reports. 6878.

https://researchrepository.wvu.edu/etd/6878

This Dissertation is protected by copyright and/or related rights. It has been brought to you by the The Research Repository @ WVU with permission from the rights-holder(s). You are free to use this Dissertation in any way that is permitted by the copyright and related rights legislation that applies to your use. For other uses you must obtain permission from the rights-holder(s) directly, unless additional rights are indicated by a Creative Commons license in the record and/ or on the work itself. This Dissertation has been accepted for inclusion in WVU Graduate Theses, Dissertations, and Problem Reports collection by an authorized administrator of The Research Repository @ WVU.

For more information, please contact researchrepository@mail.wvu.edu. 


\title{
Autism Spectrum Disorders among Adults: Burden of Healthcare Services' Utilization and Expenditures
}

\author{
Rini Vohra, B.Pharm MS \\ Dissertation submitted to the School of Pharmacy at \\ West Virginia University in partial fulfillment of the requirements for the degree of \\ Doctor of Philosophy \\ In
}

Pharmaceutical Sciences

Dr. Suresh Madhavan, Committee Chair

Dr. Usha Sambamoorthi

Dr. Nilanjana Dwibedi

Dr. Claire StPeter

Dr. Susannah Poe

Health Outcomes Research, Department of Pharmaceutical Systems and Policy, School of Pharmacy, West Virginia University

Morgantown, West Virginia 2016

Keywords: autism; healthcare utilization; autistic adults; healthcare; healthcare costs; emergency room; prescription drugs; polypharmacy; Medicaid; NEDS;

Copyright 2016 Rini Vohra 


\title{
ABSTRACT \\ Autism Spectrum Disorders among Adults: Burden of Healthcare Services' Utilization and Expenditures
}

\author{
Rini Vohra
}

\section{$\underline{\text { STUDY } 1}$}

Background: Individuals with ASD have extensive comorbidities which affect their health related quality of life. The current literature lacks documentation of prevalence of different types of comorbidities and their association with healthcare utilization and expenditures among adults with ASD as compared to adults without ASD.

Aim: To determine: 1) the differences in prevalence of psychiatric, non-psychiatric comorbidities, other health conditions, healthcare utilization, and expenditures among adults with and without ASD; and 2) the association of type of comorbidity with healthcare utilization and expenditures of adults with ASD.

Method: A retrospective cohort study using claims data from three state Medicaid Analytic eXtract was conducted. Adults aged 22-64 years with ASD (ICD9-CM code: 299.xx) with at least one inpatient or two outpatient claims on separate service dates who were continuously enrolled between January 1, 2000 to December 31, 2008 were identified. ASD cases were matched to no ASD controls by age, sex, and race using propensity score matching (1:3). Baseline and outcome differences across cases and controls were examined using bivariate analyses for categorical (chi-square) and continuous independent variables (t-tests). All cause health care utilization (outpatient visits, inpatient hospitalizations, emergency room, and prescription drug use), and associated health care costs were assessed during the follow-up year. Multivariate multinomial logistic regressions for healthcare utilization and generalized linear models with gamma distribution for expenditures were conducted to compare healthcare utilization and expenditures among adults with and without ASD. Association of type of comorbidity with healthcare utilization (binary and multinomial logistic regressions) and expenditures (generalized linear models with gamma distribution) were also analyzed among adults with ASD. Multivariate regression analyses were adjusted for state, identification year, patient socio-demographics, baseline prescription drug use, and county characteristics.

Results:

Comorbidities: Around $80 \%$ of adults with ASD $(n=1,772)$ had a psychiatric comorbidity as compared $41 \%$ of adults without ASD. Prevalence of a non-psychiatric comorbidity was significantly lower among adults with ASD (40\%ASD vs. $45 \%$ noASD). Almost $70 \%$ of adults with ASD had comorbid intellectual disability. Adults with ASD had higher rates of anxiety (12.2\% ASD vs. 5.6\% noASD), attention deficit disorders/attention deficit hyperactivity disorders (ADD/ADHD) (8.2\% ASD vs. $0.7 \%$ noASD), schizophrenia (16.6\% ASD vs. 11.1\% noASD), and gastrointestinal disorders (12.6\% ASD vs.11\% noASD) as compared to adults without ASD.

Rates of other health conditions that were significantly higher among adults with ASD as compared to adults without ASD were: epilepsy (22\%ASD vs. 5\% noASD), thyroid disease (5\%ASD vs. $1 \%$ noASD), lipid metabolism disorders (5\%ASD vs. 3\%noASD), other endocrine/nutrition disorders (11\%ASD vs. 4\% noASD) hematological disorders (7\%ASD vs. $5 \%$ noASD), genito-urinary disorders (10\%ASD vs. $8 \%$ noASD), infections (22\%ASD vs. $15 \%$ noASD), skin disorders (21\%ASD vs. $9 \%$ noASD), and paralysis (6\%ASD vs. $3 \%$ noASD). Adults with ASD also had higher prevalence rate of other disabilities such as blindness (5\%ASD vs. $4 \%$ noASD) and hearing impairments (19\%ASD vs. $4 \%$ noASD).

Healthcare utilization and expenditures: The mean number of OT visits (32ASD vs. 8noASD) and Rx claims (51ASD vs. 24noASD) per year were significantly greater for adults with ASD as compared to adults without ASD. The mean average length of stay was also higher for adults with ASD (10ASD vs. 8noASD days). Adults with ASD exhibited higher mean annual OT (\$4,375 ASD vs. \$824 noASD), ER (\$15,929 ASD vs. \$2,598 noASD), Rx (\$6,067 ASD vs. \$3,144 noASD) and total expenditures $(\$ 13,700$ ASD vs. $\$ 8,560$ noASD). 
Adults with ASD were two times more likely to have $>=4$ OT visits per year as compared to adults without ASD $(\mathrm{AOR}=2.73,95 \% \mathrm{CI}=2.31-3.22)$, five times more likely to have $>=18 \mathrm{Rx}$ claims than adults without ASD (AOR $=4.79,95 \% \mathrm{CI}=4.09-5.60)$. The likelihood of $>1 \mathrm{IP}$ visit $(\mathrm{AOR}=0.70,95 \% \mathrm{CI}=0.51-0.96)$ or $>1 \mathrm{ER}$ visit $(\mathrm{AOR}$ $=0.76,95 \% \mathrm{CI}=0.61-0.93$ ) in a year was $30 \%$ and $24 \%$ lower among adults with ASD as compared to adults without ASD. Among users, the expenditures for adults with ASD were significantly higher for OT visits, ER visits, and Rx claims as compared to adults without ASD after adjusting for selected characteristics. Adults with ASD had additional \$824 OT expenditures, \$8,932 ER expenditures, and \$1,499 Rx expenditures per year as compared to adults without ASD. The average adjusted total Medicaid expenditures (sum of OT, RX, and IP) were $\$ 1,159$ higher per year for adults with ASD as compared to adults without ASD, even after controlling for type of comorbidity. On an average, presence of a psychiatric and a non-psychiatric comorbidity among adults with ASD costed an additional $\$ 4,952$ and $\$ 5,084$ per year.

Conclusion:

Psychiatric comorbidities such as intellectual disabilities, anxiety, ADD/ADHD, and schizophrenia are very common among adults with ASD and non-psychiatric comorbidities such as gastrointestinal disorders are also highly common. Other than major comorbidities, other health conditions such as epilepsy, thyroid, lipid metabolism disorders, genito-urinary disorders, skin disorders, and infections are also highly prevalent in adults with ASD as compared to adults without ASD. Adults with ASD exhibit excess healthcare utilization in form of higher number of outpatient visits and prescription drug claims in a year and annual total Medicaid expenditures as compared to adults without ASD. Medicaid coverage of healthcare services for adults with ASD is substantial and state programs bear high costs of such services.

\section{$\underline{\text { STUDY } 2}$}

Background: The use of emergency department (ED) visits among adults with psychiatric needs is known to be very high, yet there are no comprehensive studies that have characterized ED use among adults with autism spectrum disorders (ASD). Due to the lack of clarity on adult ASD treatment guidelines and possible delayed/foregone care in outpatient settings, adults with ASD may be at a substantial risk of high ED use and costs.

Objectives: 1) To examine the trends in ED visits and mean total ED charges for adults with ASD over a period of 6 years; 2) To examine and compare the types of ED use (psychiatric, non-psychiatric, and injury) among adults with and without ASD.

Methods: A cross-sectional study using discharge level data from the Nationwide Emergency Department Sample (NEDS; 2006-2011) was conducted to the examine trends and characteristics of ED visits among adults aged 22-64 years, with and without ASD (ICD9-CM code: 299.xx). ASD cases were matched to no ASD controls by age and gender using propensity score matching (1:3). Trends are presented as annual weighted rates and mean total charges for all, psychiatric (principal diagnosis of a psychiatric disorder), non-psychiatric (principal diagnosis of a nonpsychiatric disorder), and injury (any) visits among adults with ASD. Chi square analyses and t-tests were conducted to examine sub group differences in socio-demographic, hospital, and patient disposition characteristics across adults with and without ASD. Weighted rates of psychiatric, non-psychiatric, and injury visits were compared between adults with and without ASD. Multivariate adjusted logistic regressions were run to examine the likelihood of a psychiatric, non-psychiatric, and an injury visit among adults with ASD as compared to adults without ASD. All analyses adjusted for the NEDS complex survey design.

Results: Around $0.4 \%(\mathrm{n}=25,527)$ of ED visits were associated with an adult ASD diagnosis in the unmatched sample. The rates of ED visits among adults with ASD more than doubled from 2006 to 2011 (10,047 to 23,992 per 100,000 admissions). Around one-third of the ED visits among adults with ASD led to an inpatient hospitalization. The rates of specific ED visits among adults with and without ASD were: psychiatric (15\%ASD vs. 4.2\%noASD), non-psychiatric (16\%ASD vs. $14 \%$ noASD), and injury (24\%ASD vs. $28 \%$ noASD). Most common ED visits associated with adults with ASD were: epilepsy (8.8\%), falls (6.5\%), schizophrenia (3.8\%), and respiratory disorders $(3.8 \%)$. Adults with ASD were found to be more likely to have a psychiatric visit $(\mathrm{AOR}=2.63,95 \% \mathrm{CI}=$ $2.41-2.88)$, a non-psychiatric visit $(\mathrm{AOR}=1.07,95 \% \mathrm{CI}=1.01-1.14)$, and an injury visit $(\mathrm{AOR}=1.10,95 \% \mathrm{CI}=$ 
1.04-1.16) as compared to adults without ASD. Mean total ED charges for adults with ASD were 2.3 times higher than adults without ASD.

Conclusion: Rates of ED visits among adults with ASD are on the rise. Adults with ASD use ED for different reasons which are not restricted to psychiatric needs, but also extend to non-psychiatric needs and injuries. ED visits among adults with ASD are also associated with significantly greater hospitalization rates as compared to adults without ASD. Mean total ED charges for adults with ASD are almost twice as high as charges for adults without ASD. Prevalence, risk factors, and burden of ED utilization among adults with ASD is understudied and future studies should examine the reasons and impact of such ED utilization on long term healthcare costs.

\section{$\underline{\text { STUDY } 3}$}

Background: Lack of absolute gold standard ASD treatment, approved guidelines, and strong evidence based treatments lead to greater off label prescribing with no information on the long term effects of high prescription drug use among individuals with ASD. Extent of general (six or more drug classes in a year) and psychotropic (three or more psychotropic drug classes within a period of 90 days) polypharmacy among adults with ASD has not been studied till date.

Aim: 1) To examine and compare the types of prescription drug use and rates of general and psychotropic polypharmacy among adults with and without ASD; 2) To examine predictors of polypharmacy among adults with ASD.

Method: A retrospective cross sectional study using claims data from three state Medicaid Analytic eXtract was conducted. Adults aged 22-64 years with ASD (ICD9-CM code: 299.xx) who were continuously enrolled between January 1, 2000 to December 31, 2008 were identified. ASD cases were 1:3 matched to no ASD controls by age, sex, and race using propensity score matching. Rates of different prescription drug classes, general polypharmacy, and psychotropic polypharmacy among adults with and without ASD were compared using chi-square tests. T-tests were conducted to examine the differences in mean annual number of claims for specific drug classes between adults with and without ASD. Multivariate multinomial logistic regressions were run to examine the likelihood of general and psychotropic drug polypharmacy among adults with ASD as compared to adults without ASD, after adjusting for identification year, patient socio-demographics, county characteristics, and number of comorbidities.

Results: Almost 70\% of adults with ASD had more than 25 prescription drug (Rx) claim in the identification year as compared to $28 \%$ of adults without ASD. Around $85 \%$ of adults with ASD used psychotropic drugs as compared to $42 \%$ of adults without ASD. The rates of specific psychotropic drug classes were: antipsychotics (66\%ASD vs. $20 \%$ noASD), anticonvulsants (59\%ASD vs. 20\%noASD), antidepressants (37\%ASD vs. 26\%noASD), anxiolytics/hypnotics/sedatives (21\%ASD vs. 11\%noASD), and CNS stimulants (1.9\%ASD vs. 1\%noASD). Around half of adults with ASD used six or more Rx classes per year as compared to one-third of adults without ASD. Among adults with ASD who used six or more Rx classes per year, the most common classes were: antipsychotics (78\%), anticonvulsants (75\%), antimicrobials (67\%), dermatologic agents (64\%), respiratory agents (59\%), and gastrointestinal agents (52\%). A substantial proportion of adults with ASD (19\%) also engaged in psychotropic polypharmacy as compared to adults without ASD (6\%). After adjusting for selected characteristics, adults with ASD were $17 \%$ more likely and $40 \%$ more likely to engage in general and psychotropic polypharmacy, respectively, as compared to adults without ASD.

Conclusion: Prescription drug use, general polypharmacy, and psychotropic polypharmacy is significantly and substantially higher among adults with ASD than adults without ASD. Half of adults with ASD use six or more drug classes in a year and $19 \%$ of them use three or more psychotropic drug classes within a 90 day period. Higher prescription drug use among adults with ASD was not restricted to psychotropic drugs, but extended to prescriptions alleviating other medical needs including dermatologic agents, respiratory agents, cardiovascular agents, gastrointestinal agents, antiparkinsonian agents, and many others. The study highlights considerable pharmaceuticals use by adults with ASD as well as practice of off-label prescriptions for adults with ASD by healthcare providers. Even though various clinical trials have provided with off-label options for pharmacological treatment of adults with 
ASD, such treatments should still be administered based on case by case analysis with due consideration for riskbenefit ratios. 


\section{Table of Contents}

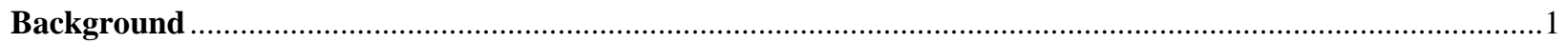

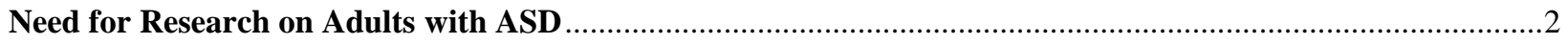

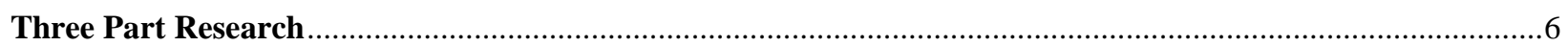

Figure 1. Three Part Research for Assessing Healthcare Burden for Adults with and without ASD ................6

Study 1. The Burden of Comorbidities, Healthcare Utilization, and Expenditures in Adult Fee-for-Service Medicaid Enrollees with and without Autism Spectrum Disorders (ASD) .

Aim: To determine the differences in prevalence of psychiatric, non-psychiatric comorbidities, other health conditions, healthcare utilization, and expenditures among adults with and without ASD. To determine the association of type of comorbidity on healthcare utilization and expenditures of adults with ASD.

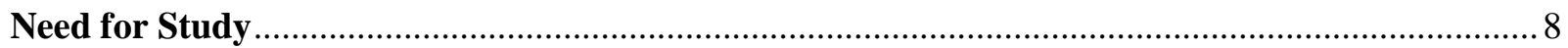

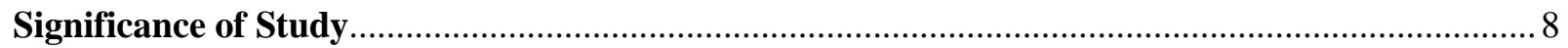

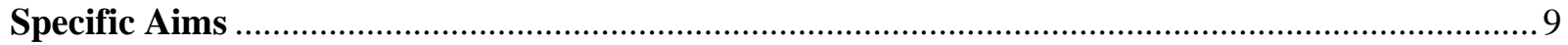

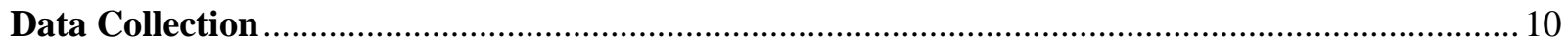

Study 2. The Burden of Emergency Department (ED) Use among Adults with and without ASD ...................12

Aim: To examine the trends and types of ED use among adults with ASD as compared to adults without ASD over a period of 6 years. To determine the economic burden of ED use among adults with ASD as

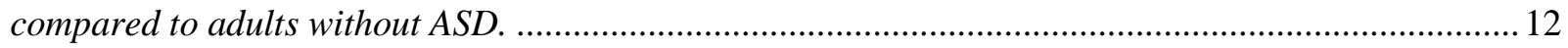

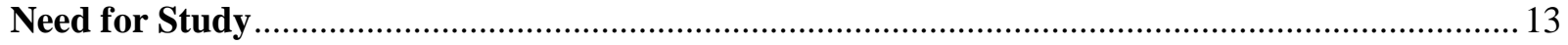

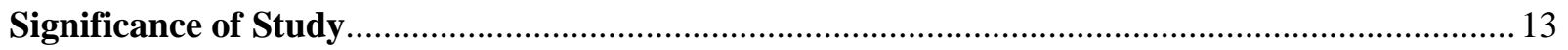

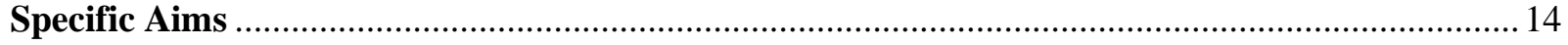

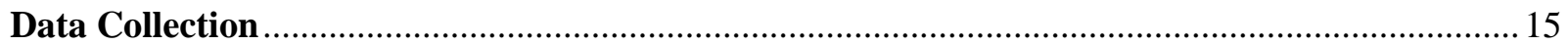

Study 3. The Burden of Medication Use in Adult Fee-for-Service Medicaid Enrollees with and without Autism Spectrum Disorders.

Aim: To determine the difference in rates of overall and specific prescription drug use along with general and psychotropic polypharmacy among adults with and without ASD. To examine the predictors of general and psychotropic polypharmacy among adults with ASD ................................. 16

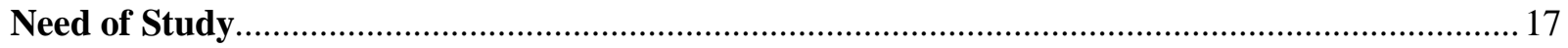

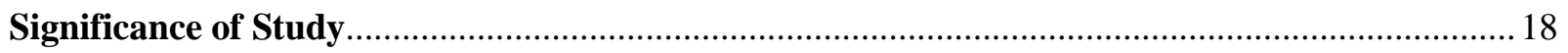

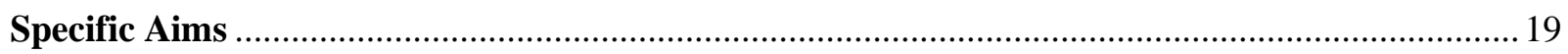

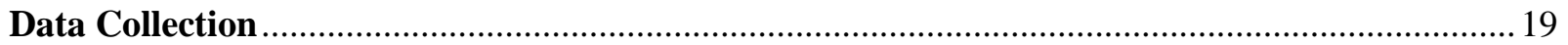

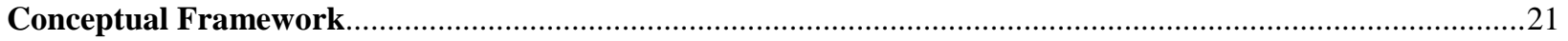

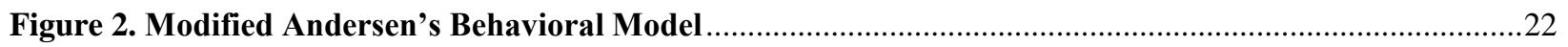

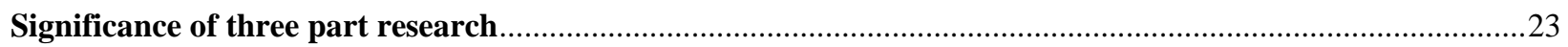

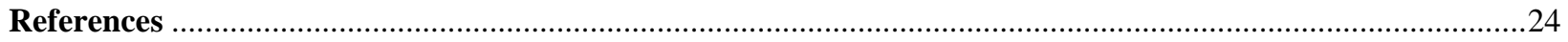

Study 1. The Burden of Comorbidities, Healthcare Utilization, and Expenditures in Adult Fee-for-Service Medicaid Enrollees with and without Autism Spectrum Disorders (ASD) .....................................................38

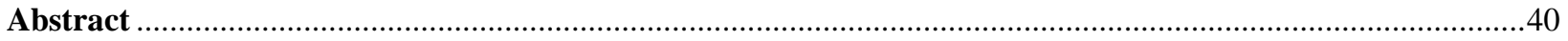

Results: 


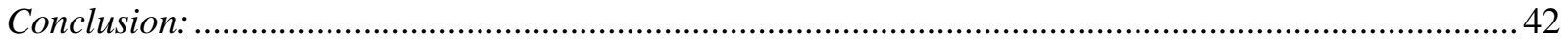

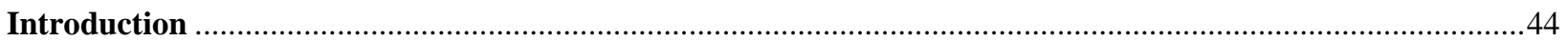

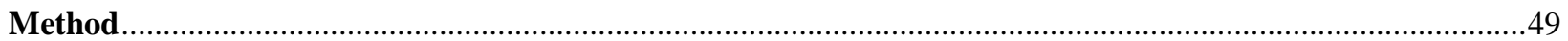

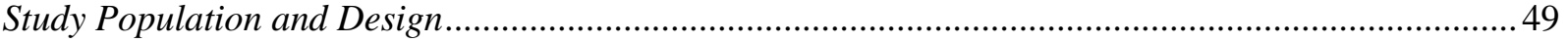

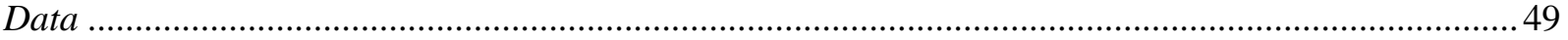

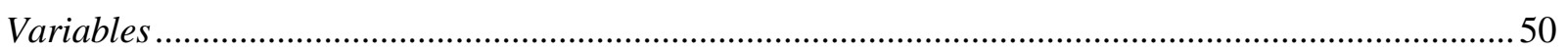

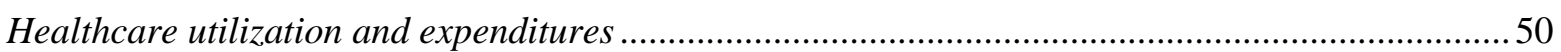

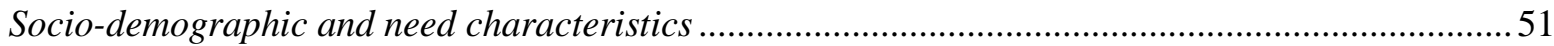

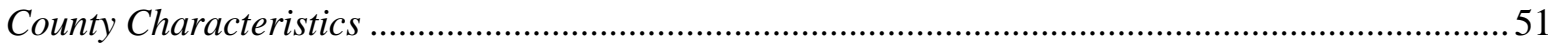

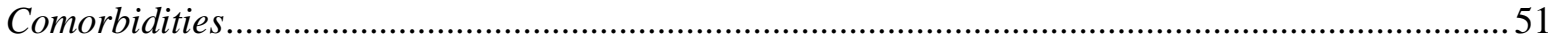

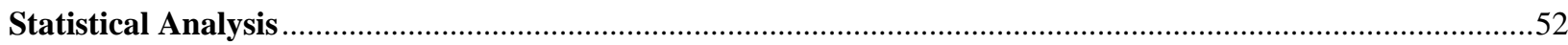

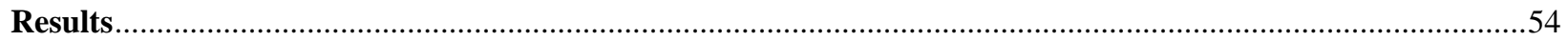

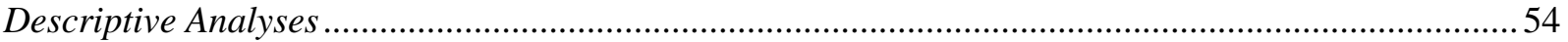

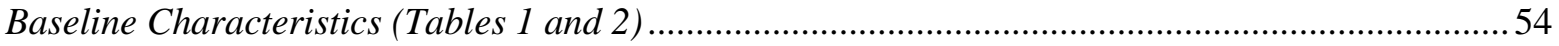

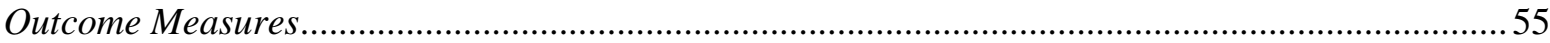

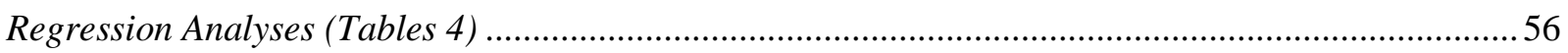

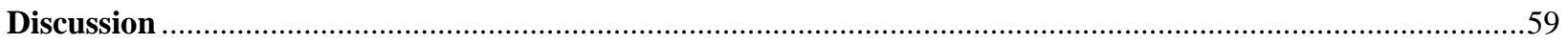

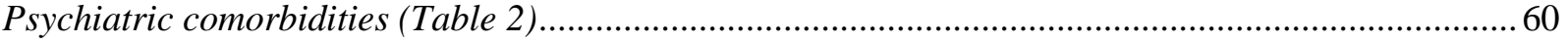

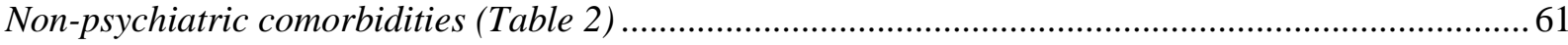

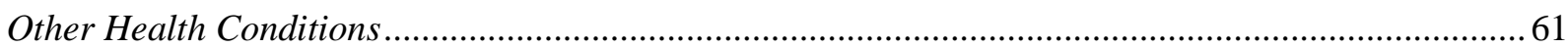

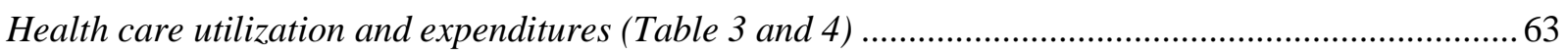

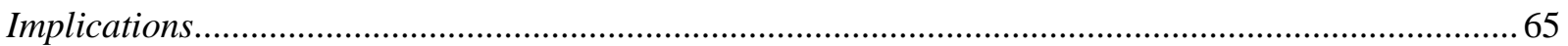

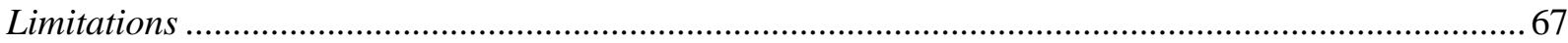

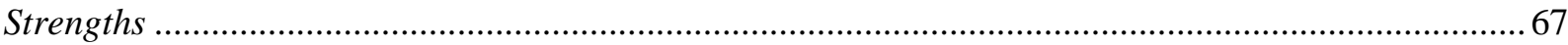

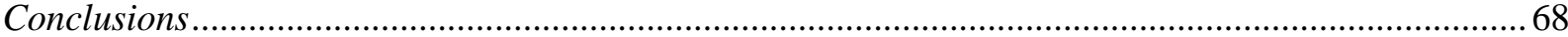

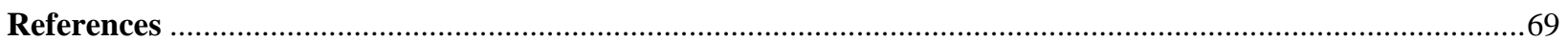

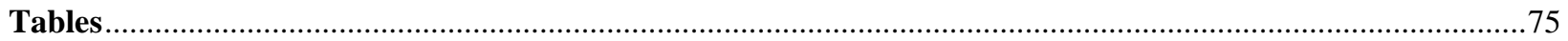

Study 2. The Burden of Emergency Department (ED) Use among Adults with and without ASD ..................84

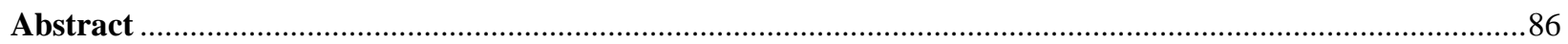

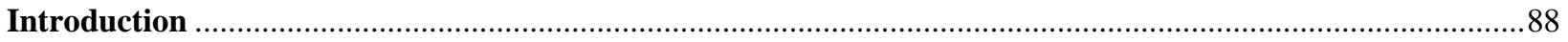

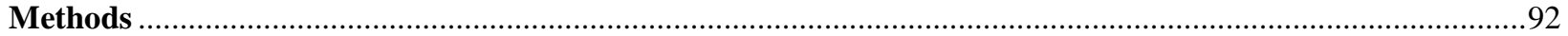

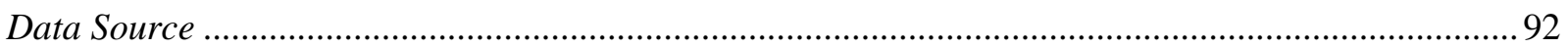

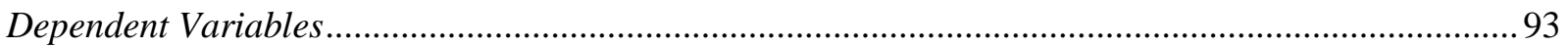

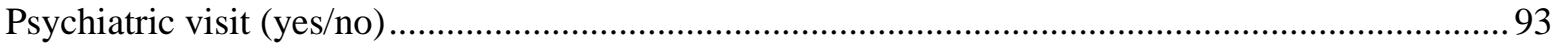

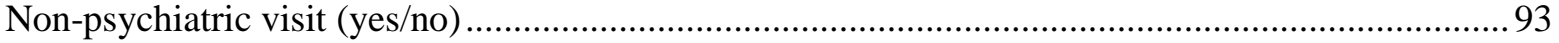

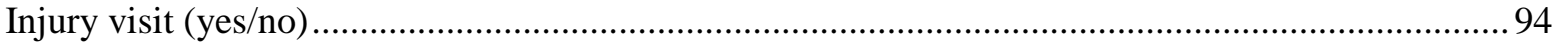

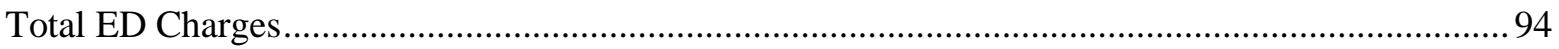

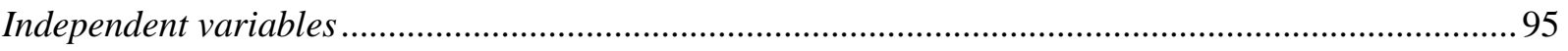




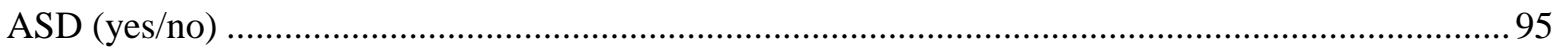

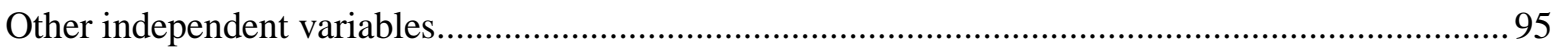

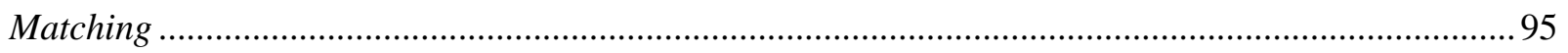

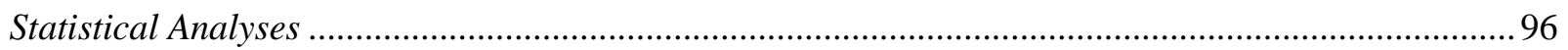

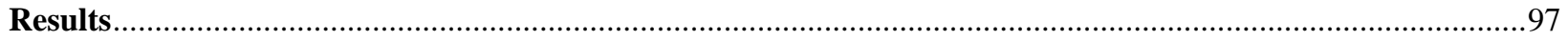

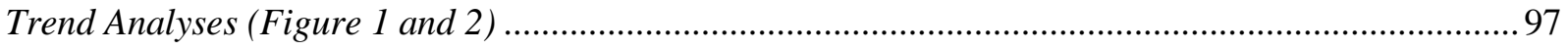

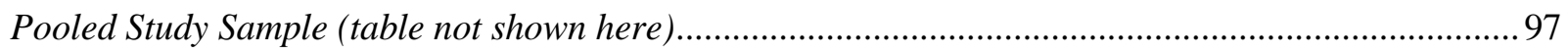

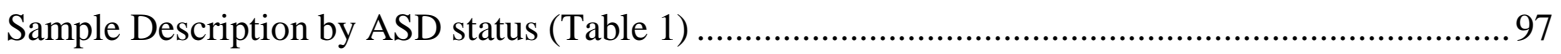

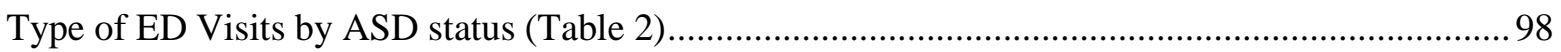

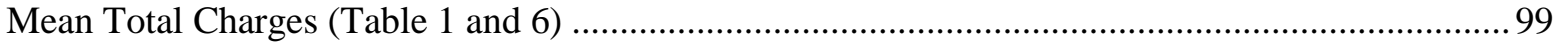

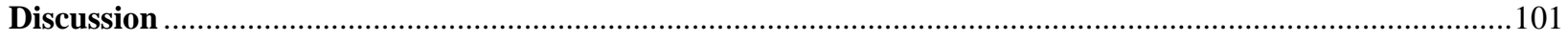

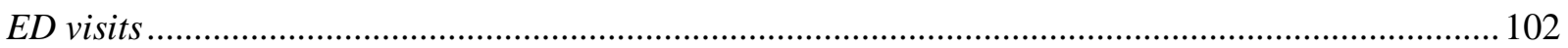

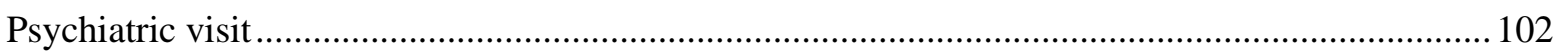

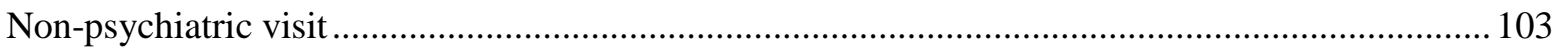

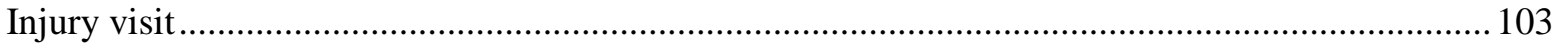

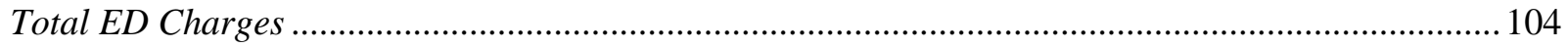

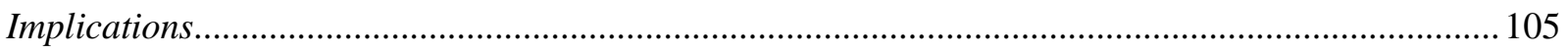

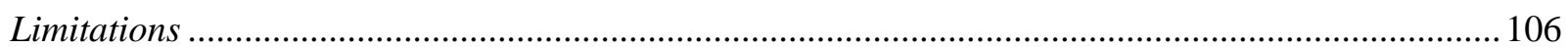

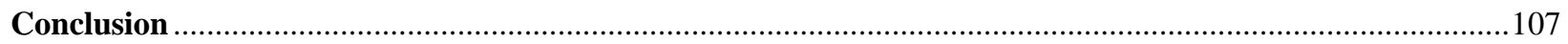

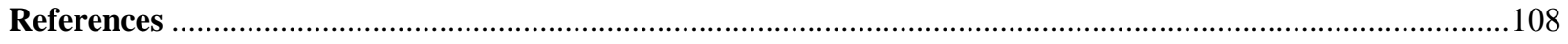

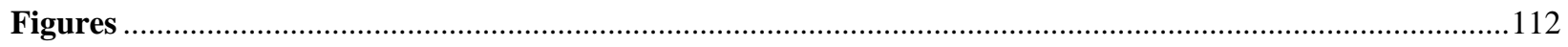

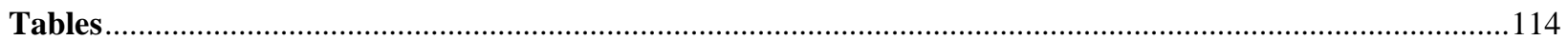

Study 3. The Burden of Medication Use in Adult Fee-for-Service Medicaid Enrollees with and without

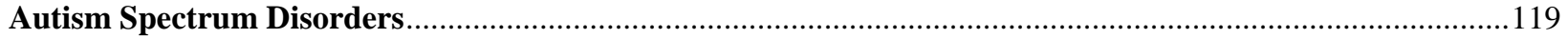

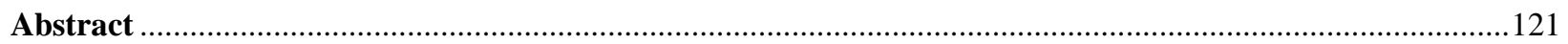

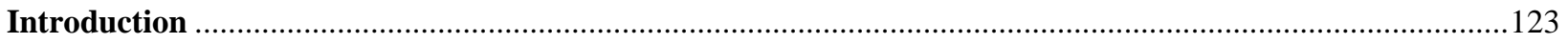

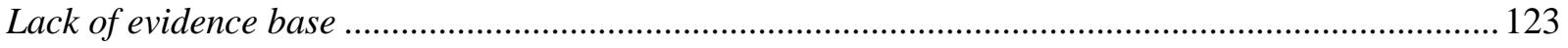

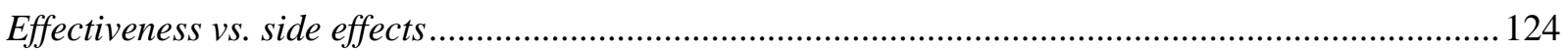

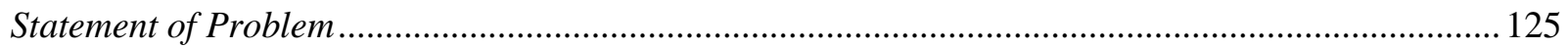

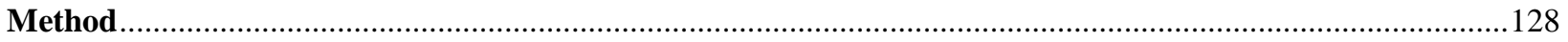

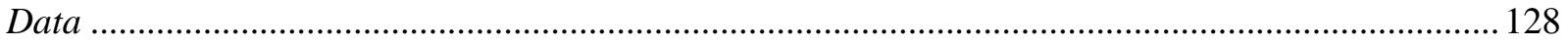

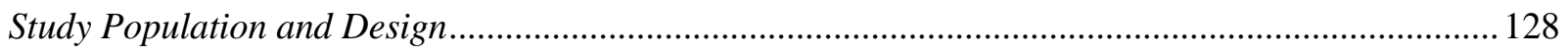

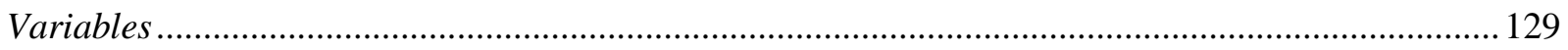

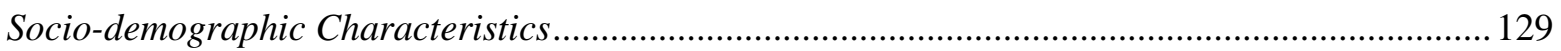

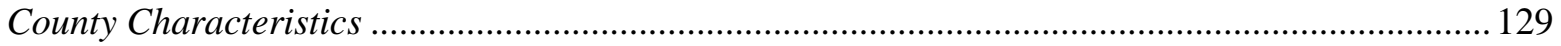

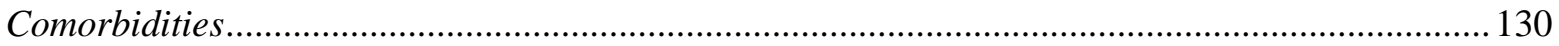

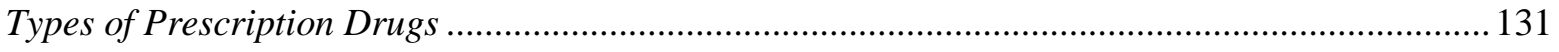

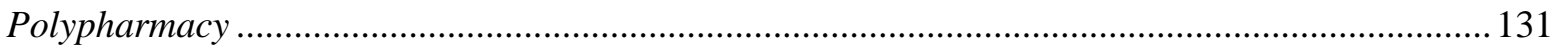

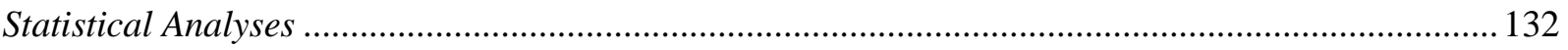




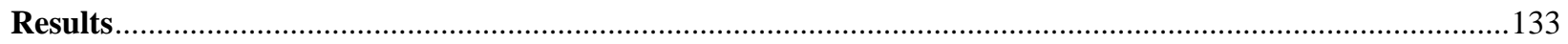

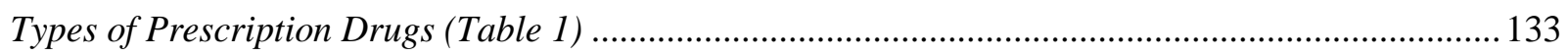

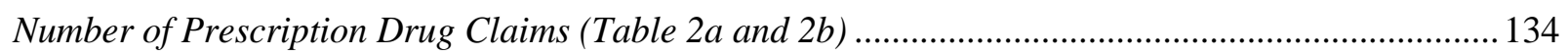

General Polypharmacy (Six or more drug classes per year; Table 1 and Table 3) ............................ 134

Psychotropic Polypharmacy (Three or more psychotropic drug classes within a 90 day period; Table 1

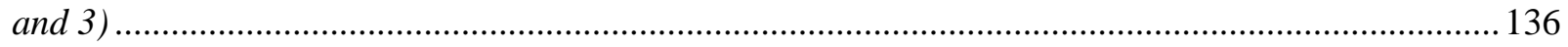

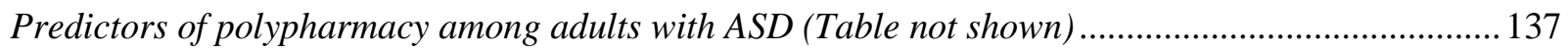

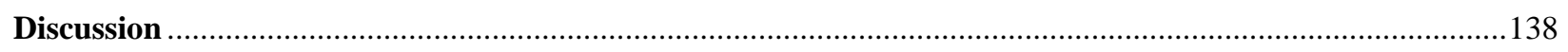

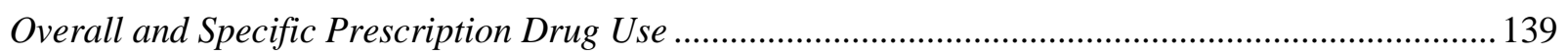

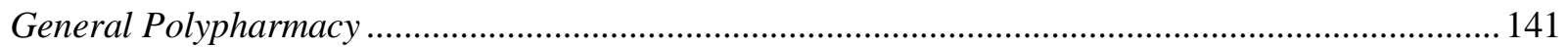

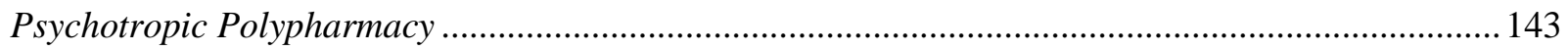

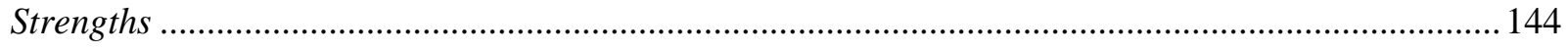

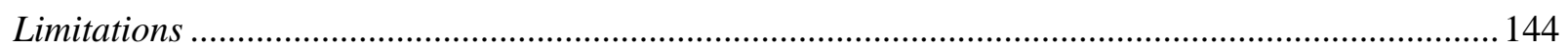

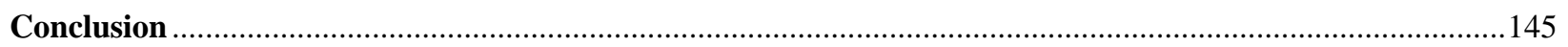

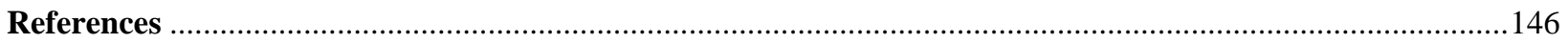

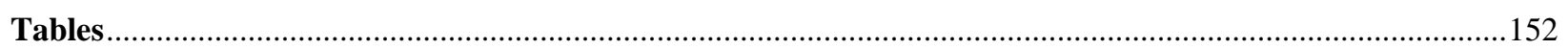

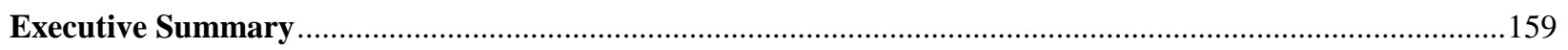

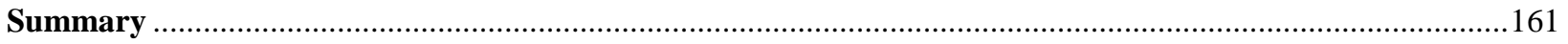

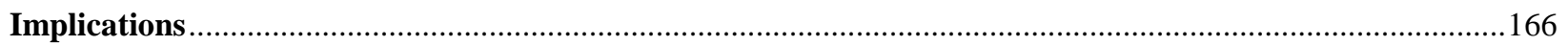

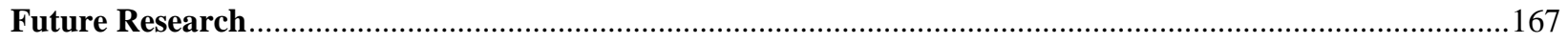

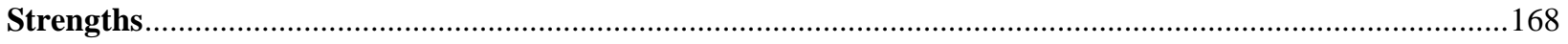

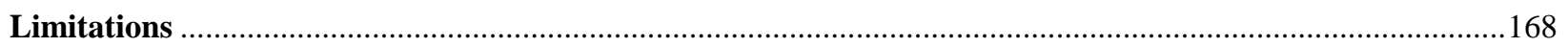

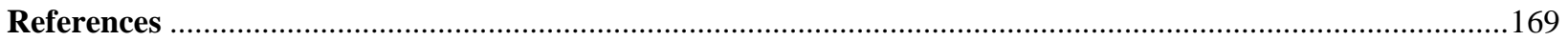




\section{Acknowledgements}

I, Rini Vohra, would like to thank God and my family members including my mother Ms. Suman Vohra and father Mr. Kundan Lal Vohra for their constant support throughout my life as well as during this phase of research. I would like to mention the immense support provided by my lovely husband Ram Shrivastava, who was there to help me out at each and every step of this $\mathrm{PhD}$ process. My gratitude also extends to my little brother Samarth Vohra and sister Garima Vohra. I would like to thank my friends and colleagues Mayank Ajmera and Amruta Manke who were there to help me through the highs and lows of PhD. Finally, I am very thankful to my committee members who provided me with critical revisions and helped improve this research work as much as possible. 


\section{Background}

Autism Spectrum Disorders (ASD) is a lifelong neuro-developmental disability

associated with impaired functioning skills and restricted, repetitive, and stereotyped patterns of behavior (American Psychiatric Association, 2000). Even though diagnostic statistical manual V (2013) provides a single definition of ASD with three levels of severity, in our study we define ASD as anyone with autistic disorder, Asperger's syndrome, pervasive developmental disorders - not otherwise specified (PDD-NOS), and other rare PDDs (ICD9-CM code: 299.xx). This is because the study period for our three part research is restricted till the year 2011, when the new diagnostic criteria were not in effect. Many individuals with ASD experience multiple challenging behaviors (Jang, Dixon, Tarbox, \& Granpeesheh, 2011; Matson \& Rivet, 2008; Murphy et al., 2005) and intellectual disabilities (Kraijer, 1999; La Malfa, Lassi, Bertelli, Salvini, \& Placidi, 2004; Morgan et al., 2002) which hamper their communicating, social, and learning capabilities (Harris \& Handleman, 1990; Kanner, 1943; Tonge \& Brereton, 2011). In addition to the core symptoms of ASD, individuals with the condition are also affected by extensive psychiatric (Hofvander et al., 2009a; Leyfer et al., 2006; Lugnegard, Hallerback, \& Gillberg, 2011a; van Steensel, Bogels, \& de Bruin, 2013) and non-psychiatric comorbidities (Buie et al., 2010; Fombonne, 2003; Horvath, Papadimitriou, Rabsztyn, Drachenberg, \& Tildon, 1999). Prevalence of Autism Spectrum Disorders (ASD) has increased considerably over the past few decades in the Unites States (Developmental Disabilities Monitoring Network Surveillance Year 2010 Principal Investigators \& Centers for Disease Control and Prevention (CDC), 2014) as well as in other countries (Lazoff, Zhong, Piperni, \& Fombonne, 2010). Among children, every 1 in 68 children have ASD in the US States (Developmental Disabilities Monitoring Network Surveillance Year 2010 Principal Investigators \& Centers for Disease Control and Prevention (CDC), 2014). Although, the prevalence of ASD among adults is 
unknown in the US, every 1 in 100 adults are known to suffer from this condition in the UK (Brugha et al., 2011).

Individuals with ASD have considerable healthcare needs and exhibit greater use of healthcare services and costs as compared to individuals without ASD (Autism Speaks, 2012; Bitsko et al., 2009; Gurney, McPheeters, \& Davis, 2006; Knapp, Romeo, \& Beecham, 2009; Liptak, Stuart, \& Auinger, 2006; Ruble, Heflinger, Renfrew, \& Saunders, 2005; Siklos \& Kerns, 2006; Wang \& Leslie, 2010a). The burden on caregivers/families of individuals with ASD is also known to be extremely high, adversely affecting caregiver health related quality of life and other family health outcomes (Cidav, Lawer, Marcus, \& Mandell, 2013; Johnson, Frenn, Feetham, \& Simpson, 2011; Khanna et al., 2011; Kogan et al., 2008; Wang \& Leslie, 2010b).

\section{Need for Research on Adults with ASD}

Autistic features persist into the adulthood and continue to manifest symptoms of diagnostic autism (Billstedt, Gillberg, \& Gillberg, 2007; Seltzer et al., 2003). Similar to children, adults with ASD also have greater healthcare needs (Billstedt, Gillberg, \& Gillberg, 2005; Magiati, Tay, \& Howlin, 2014). Previous research has mostly focused on assessing healthcare and economic outcomes for children with ASD (Cidav et al., 2013; Croen, Najjar, Ray, Lotspeich, \& Bernal, 2006; Mandell et al., 2008a). A systematic review (Mukaetova-Ladinska, Perry, Baron, Povey, \& Autism Ageing Writing Group, 2012a) found that most of the research studies concerning ageing in autism have been restricted to case reports and review articles which have discussed problems with diagnosis of autism in older adults (van Rooijen, 2005), clinical symptomatology of autism in older individuals (Lugnegard, Hallerback, \& Gillberg, 2011b; Matson \& Rivet, 2008), psychiatric comorbidities and their impact on adults with autism 
(Lugnegard et al., 2011b; Unenge Hallerback, Lugnegard, \& Gillberg, 2012), and clinical presentations and assessments of elderly cases with autism (van Niekerk et al., 2011).

With a substantial number of children with ASD transitioning to adulthood and more number of individuals receiving their "first ever" adult ASD diagnosis than ever before (American Psychiatric Association, 2012; MESIBOV \& HANDLAN, 1997; Vanbergeijk, Klin, \& Volkmar, 2008), very little is known about the healthcare needs, healthcare services' use and expenditures among adults with ASD. Therefore, studies that investigate the patterns of healthcare use, extent of healthcare utilization, and healthcare expenditures among adults with ASD are required to understand the healthcare needs of this population (Lunsky et al., 2009; Mandell, 2008; Mouridsen, Bronnum-Hansen, Rich, \& Isager, 2008; Tsakanikos, Costello, Holt, Sturmey, \& Bouras, 2007).

In addition to the tremendous gap in literature about healthcare needs and services use among adults with ASD (Shattuck et al., 2012), there are several other reasons for examining the healthcare and economic outcomes of adults with ASD:

a) Adult ASD is a complex issue because of constantly changing diagnosis criteria (Huerta, Bishop, Duncan, Hus, \& Lord, 2012). There are a certain proportion of individuals who receive ASD diagnosis later in adulthood. (Marriage, Wolverton, \& Marriage, 2009) reviewed medical charts to examine differences in adult ASD functioning by age of diagnoses (diagnosed as a child, youth, or adult). The study found that adult functioning is more variable and difficult to predict due to the changing evaluation criteria which may lead to varying healthcare outcomes for adults as compared to the younger groups, due to differences in receipt of care at the right time. 
b) Many studies have shown that healthcare providers (physicians, psychologists, psychiatrists, and mental health specialists) report lack of self-perceived competency and diagnostic abilities when treating adults with ASD (Bruder, Kerins, Mazzarella, Sims, \& Stein, 2012; Golnik, Ireland, \& Borowsky, 2009; Oskoui \& Wolfson, 2012). Also, there is a lack of clarity in appropriate adult ASD treatment and referral guidelines in the US making the prognosis complex and difficult for both the patients and the providers (Bruder, Kerins, Mazzarella, Sims, \& Stein, 2012; Golnik, Ireland, \& Borowsky, 2009; Heidgerken, Geffken, Modi, \& Frakey, 2005; Oskoui \& Wolfson, 2012). In such an environment, studies on adults with ASD that establish the healthcare needs of this population are needed to help deliver better behavioral and social outcomes earlier in their lifespans. Such health outcomes may include improved activities of daily living and employment opportunities, alleviated core symptoms, diminished social difficulties, and burden for caregivers, and consequently lower healthcare costs to society and individuals with ASD.

c) There is lack of evidence base and absence of robust clinical trials for pharmacological treatment and interventions for adults with ASD (Broadstock, Doughty, \& Eggleston, 2007; Brugha, Doos, Tempier, Einfeld, \& Howlin, 2015). Variations in symptomatology (Rumsey, Rapoport, \& Sceery, 1985) imply that pharmacological studies of children are not directly transferable to older populations, and vice versa. Therefore, making intervention and treatment decisions for adults with ASD are often dealt on a case by case basis through trial and error with no evidence of treatment efficacy and real world effectiveness. 
d) Taking care of an individual with ASD is associated with very high expenditures and lifetime costs (Chambers, Kidron, Spain, \& American Institutes, 2004; Knapp et al., 2009; Leslie \& Martin, 2007; Mandell, Cao, Ittenbach, \& Pinto-Martin, 2006; Peacock, Amendah, Ouyang, \& Grosse, 2012; Wang \& Leslie, 2010a). A recent study estimated that the cost of supporting an individual with an ASD is $\$ 1.4$ million in the United States and $£ 0.92$ million (US \$1.4 million) in the United Kingdom (Buescher, Cidav, Knapp, \& Mandell, 2014).

e) Adults with ASD report lower satisfaction with their physicians and greater unmet healthcare needs as compared to adults without ASD (Nicolaidis et al., 2013; Pellicano, Dinsmore, \& Charman, 2014). Although very few studies have examined the issue of dissatisfaction for adults with ASD with healthcare services, there is a need to identify core deficits in provision of services and reasons for higher unmet needs among this group. 


\section{Three Part Research}

The proposed research work aims to address the aforementioned gaps in the literature by examining three aspects of healthcare burden (Figure 1) associated with adults with ASD. We will also compare these aspects between adults with and without ASD.

1) Burden of comorbidities and their impact on healthcare utilization and expenditures: describing the prevalence of psychiatric, non-psychiatric comorbidities, and other health conditions among adults with and without ASD and their impact on healthcare use and expenditures;

2) Burden of emergency department (ED) use: describing 6 year ED trends, types of ED visits (psychiatric, non-psychiatric, and injury), and associated total ED charges; and

3) Burden of medication use (general and psychotropic polypharmacy)

Figure 1. Three Part Research for Assessing Healthcare Burden for Adults with and without ASD

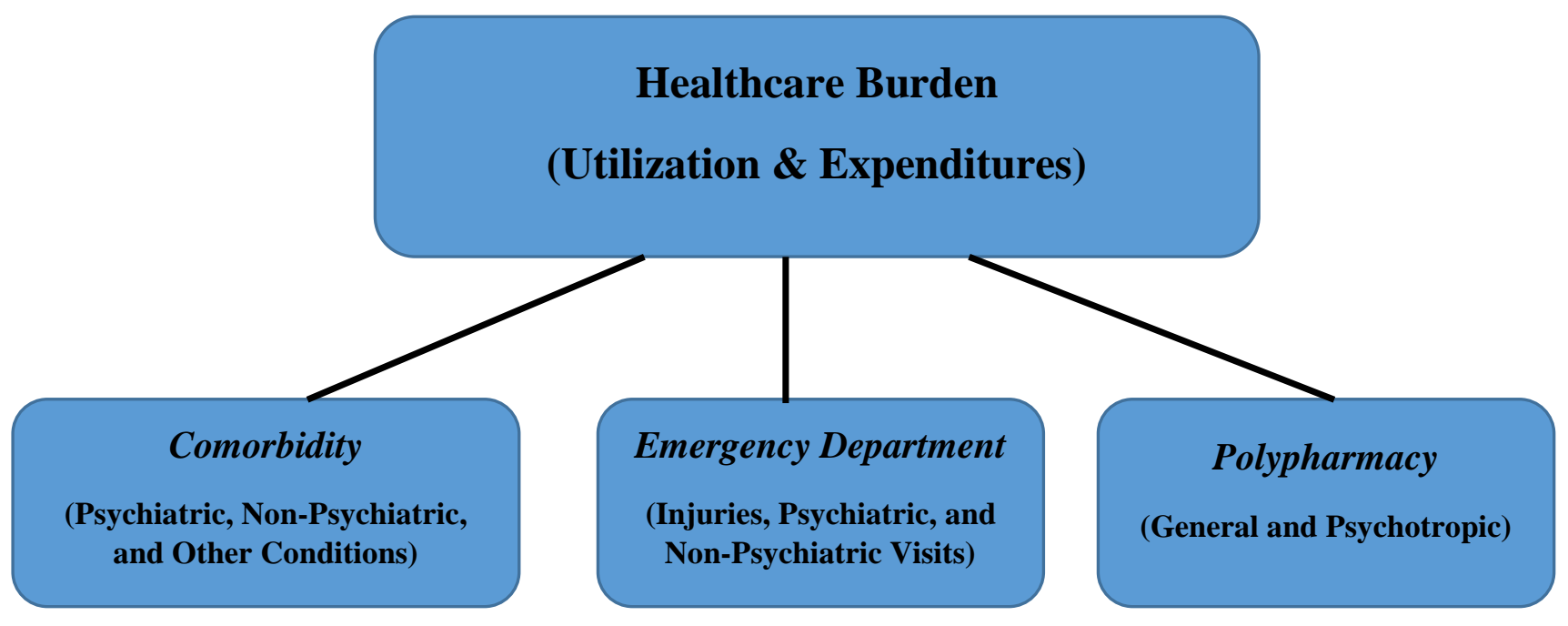




\section{Study 1. The Burden of Comorbidities, Healthcare Utilization, and Expenditures in Adult Fee-for-Service Medicaid Enrollees with and without Autism Spectrum Disorders (ASD)}

Aim: To determine the differences in prevalence of psychiatric, non-psychiatric comorbidities, other health conditions, healthcare utilization, and expenditures among adults with and without $A S D$. To determine the association of type of comorbidity on healthcare utilization and expenditures of adults with ASD.

It is well known that individuals with ASD are affected by numerous psychiatric and nonpsychiatric comorbidities that have a negative impact on their health-related quality of life (Buie et al., 2010; Khanna et al., 2011; Levy et al., 2010; Lugnegard et al., 2011a; Mandell et al., 2006; Maski, Jeste, \& Spence, 2011; McElhanon, McCracken, Karpen, \& Sharp, 2014). Such comorbidities not only have an adverse effect on core autistic symptoms (Garcia-Villamisar \& Rojahn, 2013) but also on adult's healthcare needs and use (Lunsky, Gracey, \& Bradley, 2009) such as extended length of stays in the hospital (Palucka \& Lunsky, 2007). However, to the best of our knowledge, no study in the literature has outlined the comorbidity distribution and differential associations of comorbidity types with healthcare utilization and expenditures among adults with ASD.

Our study will examine the prevalence of psychiatric and non-psychiatric comorbidities among adults with ASD as compared to adults without ASD. A psychiatric comorbidity will be defined as any co-occurring diagnosis of psychiatric disorders such as adjustment disorders, alcohol use disorders (AUD), anxiety disorders, attention deficit disorders \& conduct behavior disorders (ADD), developmental disorders, mood disorders including depressive disorders and bipolar disorder, personality disorders, schizophrenia \& other psychotic disorders, substance use disorders (SUD). A non-psychiatric comorbidity will be identified as any co-occurring diagnosis of physical disorders such as cancer, cardiovascular conditions, diabetes, epilepsy, 
gastrointestinal conditions, and respiratory conditions. We will examine the association of these comorbidities with healthcare utilization and expenditures among adults with ASD as compared to adults without ASD. We hypothesize that adults with ASD will have a significantly higher prevalence of both psychiatric and non-psychiatric comorbidities as compared to adults without ASD. Both types of comorbidities will also be associated with greater healthcare utilization and expenditures for adults with and without ASD.

\section{Need for Study}

Despite few studies that have described occurrence of specific psychiatric comorbidities among adults with ASD (Buck et al., 2014a; Lugnegard et al., 2011a; Lunsky et al., 2009), there is a lack of prevalence estimates of other comorbidities such as cardiovascular conditions and diabetes which could be equally prevalent and of prime importance among adults with ASD. In addition, other studies concerning comorbidities in adults with ASD have either included very restricted sample sizes or specific populations with intellectual disabilities (ID), or included specific psychiatry clinic settings with a significant chance of referral bias. The lack of large sample sizes and restricted study environments does not allow drawing specific conclusions on burden of comorbidities.

\section{Significance of Study}

A follow up study on patients whose ASD was ascertained in the 1980s as part of Utah/UCLA Autism Epidemiologic study was conducted by (Bilder et al., 2013). The authors found a relative risk of 9.95 mortality rate among autistic patients in a time span of 20-29 years (age range $=27$ to 54 ). Some of the major causes of death were epileptic seizures (6 of 29 deaths), lethal respiratory events (9 of 29 deaths), and cardiac deaths including myocarditis, arrhythmia, and congestive heart failure, and congenital heart disease ( 5 of 29 deaths). Therefore, examining the rates of all and specific types of comorbidities is extremely important to identify 
the needs of this population and address them earlier in life, which will help reduce preventable hospitalizations and mortality events among adults with ASD. Also, since ASD usually is considered a psychiatric/neurological condition, most of the psychiatric needs may be met in mental health settings but lack of care coordination among different primary and specialty healthcare providers may lead to greater unmet medical needs. The current study aims to address such shortfall of the current literature. This study will also explain the impact of comorbidities on healthcare utilization and expenditures which could be used as a proxy representation of healthcare needs of adults with ASD. Policymakers can use this information to stress the need for better case management in primary care and specialty settings to reduce morbidity, poor prognosis, and longer length of stays in hospitals among adults with ASD.

\section{Specific Aims}

Objective 1.1: To identify the excess healthcare utilization and expenditures (outpatient, inpatient, emergency department, and prescription drug use) for adults aged 22-64 years with ASD as compared to adults without ASD.

Hypothesis 1.1: The healthcare utilization and expenditures among adults with ASD will be significantly greater as compared to adults without ASD.

Objective 1.2: To examine and compare the prevalence of psychiatric and non-psychiatric comorbidities among adults with and without ASD.

Hypothesis 1.2: The prevalence of both psychiatric and non-psychiatric comorbidities will be significantly higher among adults with ASD as compared to adults without ASD. 
Objective 1.3: To examine and compare the impact of psychiatric and non-psychiatric comorbidities on healthcare utilization and expenditures for adults with and without ASD (Total, outpatient, inpatient, emergency department, and prescription drug use).

Hypothesis 1.3: Both psychiatric and non-psychiatric comorbidities will have a greater impact on healthcare utilization and expenditures associated with adults with ASD as compared to adults without ASD.

\section{Data Collection}

We will examine the prevalence of comorbidities, healthcare utilization, and expenditures among adults with and without ASD using three state Medicaid administrative claims data (IL, NY, and TX; 2000-2008). Medicaid is the single largest payer for individuals with ASD (Ruble et al., 2005) and utilization of Medicaid provided healthcare services among individuals with ASD is only expected to increase (Semansky, Xie, \& Mandell, 2011a). Medicaid provides a wide variety of services to individuals with ASD (Arjun, Hope, Guyer, \& Alker, 2011) including providing primary and secondary health insurance coverage for healthcare services, home and community based waivers, screening and diagnostic services, behavioral support, in home care, skill training/acquisition, respite care, case management, supported employment, service coordination, self-directed services, and parent training and education (Mauch, Pfefferle, Booker, Pustell, \& Levin, 2011).

Healthcare administrative claims, especially state Medicaid databases have been extensively used for ASD research among children with validated algorithms for identification of ASD (Khanna, Jariwala, \& West-Strum, 2013; Mandell et al., 2008a; Maski et al., 2011; Peacock et al., 2012; Spencer et al., 2013). Knowing that Medicaid serves as a critical healthcare service payer for individuals with ASD, examining the health outcomes for adults with ASD using 
Medicaid claims will help provide a complete picture of healthcare utilization and expenditures as well as generate evidence for extensive healthcare needs of this population. This evidence can be used to create better state policies and expand Medicaid healthcare coverage to other essential and costly healthcare services for adults with ASD. We selected IL, NY, and TX because of lower managed care penetration rates and greater fee-for-service enrollment during the past decade (study period: 2000-2008) (The Henry J Kaiser Family Foundation, 2013). 


\section{Study 2. The Burden of Emergency Department (ED) Use among Adults with and without ASD}

Aim: To examine the trends and types of ED use among adults with ASD as compared to adults without ASD over a period of 6 years. To determine the economic burden of ED use among adults with ASD as compared to adults without ASD.

The second study will focus on an understudied, yet very critical aspect of healthcare services for adults with and without ASD. ED use is extremely high among patients with psychiatric needs (Hazlett, McCarthy, Londner, \& Onyike, 2004; Larkin, Claassen, Emond, Pelletier, \& Camargo, 2005; Mahajan et al., 2009; Sills \& Bland, 2002a). Almost 38\% of ED visits in the US are associated with a psychiatric disorder. Considering the psychiatric needs of individuals with ASD (Simonoff et al., 2008), presence of extensive comorbidities such as behavioral disturbance and conduct disorders (Matson \& Rivet, 2008), and previous studies documenting substantial ED use among children with ASD (Croen et al., 2006; Kalb, Stuart, Freedman, Zablotsky, \& Vasa, 2012), it is expected that ED use will be substantially high among the adult ASD population as well.

We will examine and compare the trends in rates of ED use and total ED charges for adults with and without ASD across a span of 6 years (2006-2011). We will also compare the rates of specific ED visits such as psychiatric, non-psychiatric, and injury visits among adults with and without ASD. A psychiatric ED visit will be identified as any visit with a principal diagnosis of a psychiatric disorder such as adjustment disorders, alcohol use disorders (AUD), anxiety disorders, attention deficit disorders \& conduct behavior disorders (ADD), developmental disorders, mood disorders including depressive disorders and bipolar disorder, personality disorders, schizophrenia \& other psychotic disorders, substance use disorders (SUD). A non-psychiatric ED visit will be identified as any visit with a principal diagnosis of a physical 
disorder such as cancer, cardiovascular conditions, diabetes, epilepsy, gastrointestinal conditions, and respiratory conditions. An injury visit will be identified as a visit associated with any injury such as: assault, cutting, drowning, fall, fire, machinery, poison, being struck, suffocation, suicidal ideation, and self-harm. We hypothesize that ED visit rates will increase over the 6 year period for adults with ASD. Adults with ASD will also be more likely to have a psychiatric and injury ED visit as well as higher total ED charges as compared to adults without ASD.

\section{Need for Study}

In the US, at least one-third of ED visits are avoidable/non-urgent/ambulatory care sensitive and treatable in primary care settings and over $\$ 18$ billion dollars are spent annually on such avoidable ED visits (Choudhry et al., 2007). Therefore, identifying and examining at high risk and frequent ED users will help reduce long term ED costs. Studying the pattern of ED use is also equally critical for adults with ASD because increasing age has been found to be associated with frequent visits to ED (Pines et al., 2011). Adults with ASD may be frequent ED users due to paucity of trained mental healthcare professionals who can meet their specific needs outside ED settings at the appropriate time. Adults with ASD may also present a different symptomatology and events to the ED as compared to adults without ASD. Hence, examination of burden of the ED use in a high needs and growing population of adults with ASD will not only address literature gaps, but will also help highlight reasons for ED use, which may reflect issues with accessing timely care, lack of trained professionals, and lack of availability of appropriate and quality care in an outpatient/primary care setting (Soto et al., 2009; E. R. Williams et al., 2001).

\section{Significance of Study}

If the proportion and costs of ED visits among adults with ASD are found to be substantially higher as compared to adults without ASD, then the healthcare providers can utilize 
this information to identify various needs (psychiatric or non-psychiatric) of adults with ASD in the primary care settings. For example, identifying psychiatric conditions which can be better managed at the outpatient level (primary care or specialty) can help reduce frequent ED visits, ED costs, inpatient hospitalizations, and readmissions for adults with ASD. In addition, we will also examine if adults with ASD who may be prone to self-injurious behaviors and conduct problems (Kato et al., 2013; Matson \& Rivet, 2008) are more likely to have an injury ED visit as compared to adults without ASD. Information on the type of injuries affecting adults with ASD will help ED staff and healthcare providers to better prepare for ASD cases entering ED. Lastly, our study will also examine the costs associated with the ED use to provide an understanding of the financial implications of ED use among adults with ASD as compared to adults without ASD.

\section{Specific Aims}

Objective 2.1: To examine and compare the trends in prevalence rates of all, psychiatric, nonpsychiatric, and injury ED visits for adults with ASD.

Hypothesis 2.1: The prevalence rates of all, psychiatric, non-psychiatric, and injury ED visits by adults with ASD are expected to significantly increase over the 6 year period.

Objective 2.2: To examine and compare the trends in mean total ED charges (all, psychiatric, non-psychiatric, and injury ED visits) among adults with ASD.

Hypothesis 2.2: The mean total ED charges for all, psychiatric, non-psychiatric, and injury ED visits by adults with ASD are expected to increase over the 6 year period.

Objective 2.3: To examine and compare the socio-demographic, hospital, patient disposition characteristics, type of visits, and average total ED charges for adults with and without ASD. 
Objective 2.4: To examine the likelihood of psychiatric, non-psychiatric, and an injury ED visit among adults with and without ASD by utilizing predictors such as presence of ASD, sociodemographic, hospital, and patient disposition characteristics.

Hypothesis 2.4: Adults with ASD will show greater likelihood of a psychiatric, non-psychiatric, or an injury ED visit after adjusting for relevant characteristics mentioned above.

\section{Data Collection}

We plan to use Nationwide Emergency Database Sample (NEDS) 2006- 2011, largest allpayer ED database which includes national estimates for hospital based ED data visits created for the Healthcare Cost and Utilization Project, Agency of Healthcare and Research Quality (Healthcare Cost and Utilization Project (HCUP), 2011). NEDS compiles discharge data collected from hospital billing records from both state emergency department databases (SEDD) and state inpatient databases (SID). The SID contains information on patients initially seen in the ED and then admitted to the same hospital. The SEDD capture information on ED visits that do not result in an admission (i.e., treat-and-release visits and transfers to another hospital).

The NEDS is built using a $20 \%$ stratified sample of institutions and collects data from 951 hospitals located in 30 States with an unweighted 30 million discharges each year. The NEDS presents rich information on type of ED visits (psychiatric, injury, etc.) along with up to 15 diagnoses associated with each visit, geographic information, hospital characteristics, and total charges for each ED visit. Using a data such as NEDS not only helps understand the extent and patterns of ED use among adults with ASD but also provides additional information on reasons for such ED visits such as type of injuries (which has not been previously reported in any study on adults with ASD), primary diagnosis for the ED visit, and the outcome(s) of the ED visits (e.g. treated and released, transfer to home health, or inpatient hospitalizations etc.). 


\section{Study 3. The Burden of Medication Use in Adult Fee-for-Service Medicaid Enrollees with and without Autism Spectrum Disorders}

Aim: To determine the difference in rates of overall and specific prescription drug use along with general and psychotropic polypharmacy among adults with and without ASD. To examine the predictors of general and psychotropic polypharmacy among adults with ASD.

*General Polypharmacy: A prescription drug claim for six or more different therapeutic classes in the identification year.

*Psychotropic Drug Polypharmacy: A prescription drug claim for 3 or more different psychotropic classes within a period of 90 days after the first prescription fill date in the identification year.

The third aim of this three part research will determine and compare the burden of any prescription drug use and psychotropic drug use among adults (22-64 years) with and without ASD. This study will also outline and compare the distribution of specific drug classes within these categories among adults with and without ASD. General polypharmacy may include but is not restricted to prescription drug classes such as antipsychotics, anti-diabetes, anti-neoplastics, antibiotics, gastrointestinal agents, respiratory agents, and many more. Psychotropic polypharmacy includes drug classes such as antipsychotics, antidepressants, CNS stimulants, anxiolytics/sedatives/hypnotics, and anticonvulsants. The study will also examine and compare the association of polypharmacy use with other healthcare utilization and expenditures among adults with ASD. In contrast to the abundant literature on general and psychotropic drug polypharmacy use in children with ASD, very few studies have examined the patterns of medication use among adults with ASD.

(Esbensen, Greenberg, Seltzer, \& Aman, 2009) examined the use of psychotropic (such as antipsychotics and anti-depressants) and non-psychotropic medications (such as anti lipimic, GI medications, and other drugs) among adolescents and adults with ASD (average age 21 years) via longitudinal (4.5 years) contingency analyses and found a high degree of likelihood to stay 
medicated (Odds: 15.83). A critical finding of the study was that once a medication was prescribed, the discontinuation over a 4.5 year period was very unlikely. (Khanna et al., 2013) examined Mississippi Medicaid fee for service claims data to assess the psychotropic medication use and costs associated among individuals with ASD. Adults with ASD (22-64 years of age) had the highest average number of claims per beneficiary for any psychotropic drug. Using literature support of such studies, we hypothesize that adults with ASD will have greater rates of general as well as psychotropic drug polypharmacy than those without ASD. This may be due to paucity of clinical trials for most prescription drugs among adults with ASD, lack of training of healthcare providers in terms of treatment guidelines (Bruder et al., 2012; Golnik et al., 2009; Heidgerken et al., 2005; Oskoui \& Wolfson, 2012), greater likelihood of adults with ASD using psychotropic medications than their younger counterparts (Khanna et al., 2013), and low likelihood of discontinuation of a medication once started at a younger age (Esbensen et al., 2009).

\section{Need of Study}

Majority of ASD literature has focused on examining the patterns of psychotropic polypharmacy among children with ASD. Even though the definition of polypharmacy has varied with studies, use of any psychotropic medication has been reported to be as high as $83 \%$, with polypharmacy being reported among 10-20\% of children with ASD (Coury et al., 2012; Frazier et al., 2011; Gerhard, Chavez, Olfson, \& Crystal, 2009; Logan et al., 2012; Mandell et al., 2008; Oswald \& Sonenklar, 2007a; Oswald \& Sonenklar, 2007b; Spencer et al., 2013).

Adults with ASD have received very little attention regarding polypharmacy use, with no documentation of the burden of any prescription drug use in this population. Even though pharmacological treatment is an absolute necessity in ASD management (Baribeau \& 
Anagnostou, 2014; Volkmar, Cook, Pomeroy, Realmuto, \& Tanguay, 1999) and helps to alleviate symptoms associated with other co-occurring conditions (Baribeau \& Anagnostou, 2014), the evidence base for most of these medications (including FDA approved antipsychotics such as risperidone and aripiprazole for ASD) have received a status of only medium to low level effectiveness for core symptoms of ASD (Warren et al., April 2011). In addition, use of such prescription drugs (e.g. antidepressants, stimulants, antipsychotics etc.) is also associated with significant adverse events and side effects such as aggression, suicidal ideation, obesity, and sedation (Blankenship, Erickson, Stigler, Posey, \& McDougle, 2010; Ching \& Pringsheim, 2012; Marcus et al., 2009; McCracken et al., 2002; Owen et al., 2009; West, Brunssen, \& Waldrop, 2009; K. Williams, Wheeler, Silove, \& Hazell, 2010). Therefore, a study documenting the overall medication burden is needed to underline the varied pharmaceutical uses in this population.

\section{Significance of Study}

Use of polypharmacy increases the possibility of drug-drug interactions which pose additional risk of adverse events aggravating the complexities of healthcare management of individuals with ASD. The results of this study can be used to describe the prescription patterns and utilization of multiple off label medications (lack of FDA approval for most medications among adults with ASD), in addition to informing physicians who in most cases report being under-trained in pharmacological treatment approaches of patients with ASD (Bruder et al., 2012; Oskoui \& Wolfson, 2012). The findings will identify which prescription drug classes are commonly used by adults with ASD and demonstrate a need of pharmacological treatment guidelines (Bruder et al., 2012; Golnik et al., 2009; Heidgerken et al., 2005; Oskoui \& Wolfson, 2012) and the needs of clinical trials for drugs commonly prescribed to adults with ASD (Broadstock et al., 2007; Brugha et al., 2015). 


\section{Specific Aims}

Objective 3.1: To examine and compare the use of different prescription drug classes among adults with and without ASD.

Hypothesis 3.1: Use of various prescription drug classes will be higher among adults with ASD as compared to adults without ASD.

Objective 3.2: To determine and compare the rates of general and psychotropic drug polypharmacy use among adults with and without ASD.

Hypothesis 3.2: Adults with ASD will show higher rates of general as well as psychotropic drug polypharmacy as compared to adults without ASD.

Objective 3.3: To determine and compare the likelihood of general and psychotropic drug polypharmacy use among adults with and without ASD.

Hypothesis 3.3: After adjusting for socio-demographics, patient characteristics, county level healthcare workforce, and Medicaid eligibility, adults with ASD will be more likely to use both general and psychotropic drug polypharmacy as compared to adults without ASD.

Objective 3.4: To identify the predictors of general and psychotropic drug polypharmacy among adults with ASD.

\section{Data Collection}

Similar to Study 1, we will use three state Medicaid administrative claims data (IL, NY, and TX; 2000-2008) to determine the rates of polypharmacy, and its association with other healthcare utilization, and expenditures among adults with ASD. Although, prescription drug coverage is an optional benefit under federal Medicaid law, all state Medicaid programs provide coverage for outpatient pharmacy prescription drugs to eligible individuals. Medicaid plays a 
significant role in prescription drug coverage for individuals with mental health and substance abuse issues as well (Schubart et al. 2014). Many studies have utilized Medicaid claims databases to examine the rates of psychotropic drug polypharmacy among children with ASD (Frazier et al., 2011a; Logan et al., 2012; Mandell et al., 2008b). Thus, using this database will help provide a comprehensive understanding of prescription drug use among adults with ASD. 


\section{Conceptual Framework}

The three studies proposed in this document will use the modified version of Andersen's socio-behavioral model (Andersen, 1995). Andersen's model has been previously used in plethora of studies and is very useful when the researchers intend to identify the reasons for excess healthcare utilization and expenditures among specific group of patients as compared to other patient groups.

We will use this model to determine the factors that contribute to the anticipated excess healthcare utilization and expenditures for adults with ASD as compared to adults without ASD. The framework constitutes individual level factors and community level factors which determine an individual's behavior, their healthcare needs, access to services, and consequently their healthcare utilization and costs. Individual level factors are divided into: predisposing (e.g. patient demographic information such as age, gender, and race), enabling (e.g. urban/rural status of place of care/residence, education level, annual household income level, and Medicaid eligibility), and need factors (e.g. comorbidities). The community level factors include contextual characteristics such as state and region that influence the nature of policies and extent of healthcare access to the individuals with and without ASD. For example, healthcare services offered under the state Medicaid programs vary substantially from state to state, depending on the state funds and other state policies (Mauch et al., 2011).

Together these factors will be used to explain differences in outcomes (healthcare utilization and expenditures) for adults with and without ASD. Such outcomes include utilization and costs of services such as outpatient visits, inpatient hospitalizations, ED, and prescription medication use. 


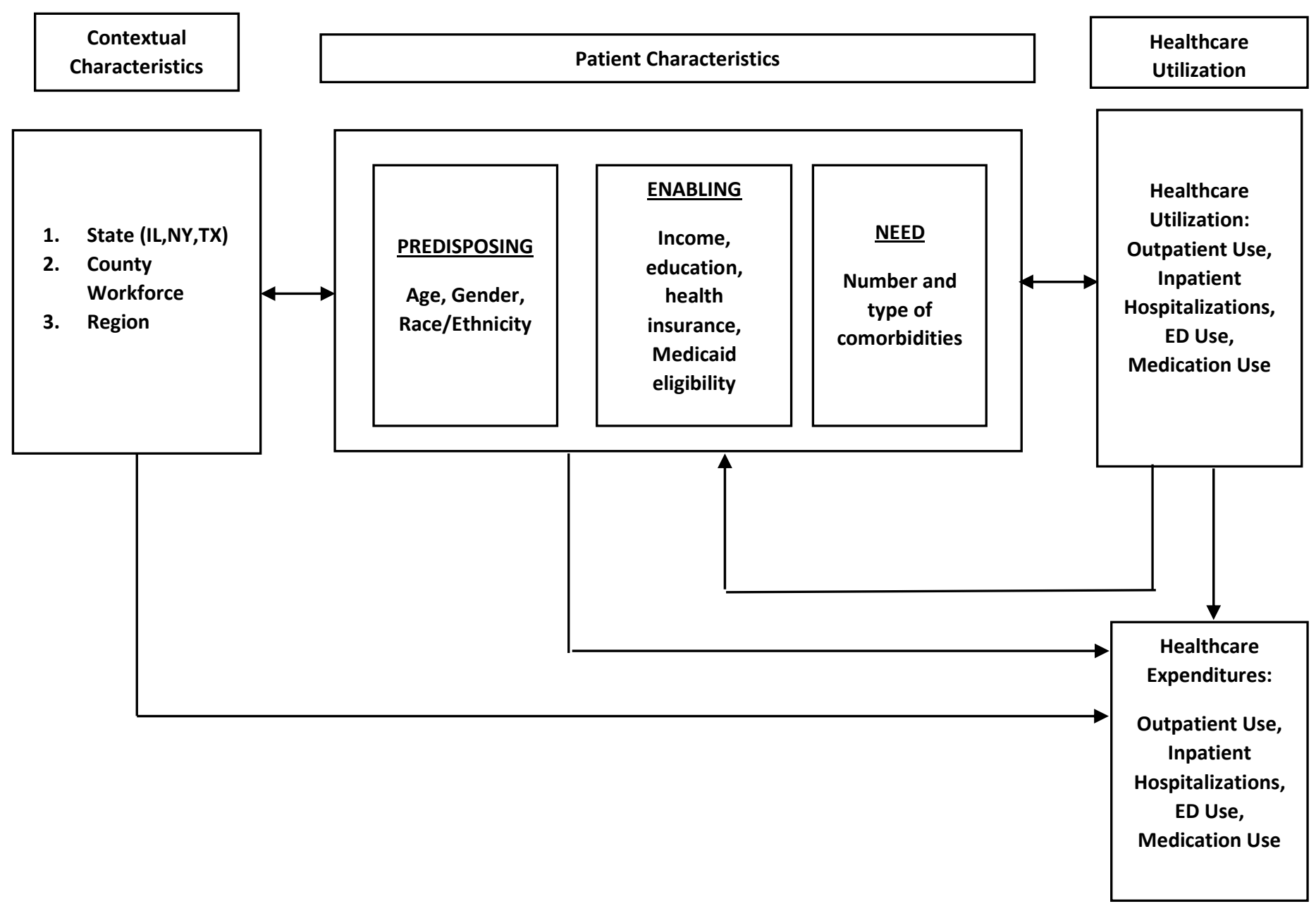

Figure 2. Modified Andersen's Behavioral Model 


\section{Significance of three part research}

The current dissertation research will fill a tremendous gap in the literature about the pattern of healthcare use and expenditures associated with adults with ASD. (Buescher et al., 2014) predicted significantly greater medical costs for adults with ASD as compared to their pediatric counterparts. Given the increasing number of ASD cases, there is a growing concern about the healthcare utilization and costs associated with the children transitioning to adults. Our research will identify the healthcare needs of adults with ASD by examining the extent of their comorbidities, overall prescription medication use and polypharmacy, and burden of ED use.

The research will highlight the nature of off label prescription drug use and perhaps, underline the need of clinical drug trials among adults with ASD. If adults with ASD have higher healthcare needs, use greater healthcare services, and have higher expenditures, the findings will help autism advocacy groups and policymakers to provide a rationale for expanding and improving the quality of healthcare coverage, encouraging ASD awareness and education for healthcare providers, and providing early intervention services to reduce long term morbidity and poor prognosis among those diagnosed as children. The findings from our study will help reduce long term healthcare costs to the payers as well as to the society. It will also help to understand the need for improving social outcomes for adults with ASD, who, when provided timely care may have better employment opportunities and work productivity.

Overall, this research may provide evidence that adults with ASD are in substantial need of healthcare services and have higher costs as compared to adults without ASD. Policymakers may need such information to create mandates/policies that can serve the specific needs of this population in a cost effective manner. 


\section{References}

Adler, B. A., Wink, L. K., Early, M., Shaffer, R., Minshawi, N., McDougle, C. J., \& Erickson, C. A. (2014). Drugrefractory aggression, self-injurious behavior, and severe tantrums in autism spectrum disorders: A chart review study. Autism : The International Journal of Research and Practice, doi:1362361314524641 [pii]

Ahmedani, B. K., \& Hock, R. M. (2012). Health care access and treatment for children with co-morbid autism and psychiatric conditions. Social Psychiatry and Psychiatric Epidemiology, 47(11), 1807-1814. doi:10.1007/s00127-012-0482-0; 10.1007/s00127-012-0482-0

American Psychiatric Association (Ed.). (2000). Diagnostic and statistical manual of mental disorders (4th ed.). Washington D.C.: American Psychiatric Publishing, Inc.

American Psychiatric Association. (2012). DSM-5 proposed criteria for autism spectrum disorder designed to provide more accurate diagnosis and treatment. (News Release No. 12-03). Arlington,VA: American Psychiatric Association.

Andersen, R. M. (1995). Revisiting the behavioral model and access to medical care: Does it matter? Journal of Health and Social Behavior, 36(1), 1-10.

Arjun, L., Hope, C., Guyer, J., \& Alker, J. (2011). Medicaid and its role for children and youth with special health care needs (CYSHCN): A family perspective. (). Georgetown University, Health Policy Institute: Center for Children and Families/Family Voices.

Armand-Branger, S., Poisson, N., Gaudoneix-Taieb, M., \& Ramos, O. (2009). Pharmaco-therapeutic evaluation of medical treatments of adult autistics and multi-handicapped patients in a public mental health unit. [Les traitements psychotropes prescrits dans un etablissement public de sante mentale accueillant des personnes atteintes d'autisme et des polyhandicapes] L'Encephale, 35(4), 370-376. doi:10.1016/j.encep.2008.04.008 [doi]

Autism Speaks. (2012). New research finds annual cost of autism has more than tripled to $\$ 126$ billion in the U.S. and reached $£ 34$ billion in the U.K. Retrieved from http://www.autismspeaks.org/about-us/pressreleases/annual-cost-of-autism-triples

Autism Speaks. (2013). High-quality early intervention for autism more than pays for itself. Retrieved from http://www.autismspeaks.org/science/science-news/high-quality-early-intervention-autism-more-pays-itself

Autism Speaks. (2015). 10 year timeline. Retrieved from https://www.autismspeaks.org/10year/timeline

Bailey, D. B.,Jr, Hebbeler, K., Scarborough, A., Spiker, D., \& Mallik, S. (2004). First experiences with early intervention: A national perspective. Pediatrics, 113(4), 887-896.

Bakare, M. O., Munir, K. M., \& Kinney, D. K. (2011). Association of hypomelanotic skin disorders with autism: Links to possible etiologic role of vitamin-D levels in autism? Hypothesis (University of Toronto.Dept.of Medical Biophysics), 9(1), e2. doi:e2 [pii]

Baker, J. P. (2013). Autism at 70--redrawing the boundaries. The New England Journal of Medicine, 369(12), 10891091. doi:10.1056/NEJMp1306380; 10.1056/NEJMp1306380

Baribeau, D. A., \& Anagnostou, E. (2014). An update on medication management of behavioral disorders in autism. Current Psychiatry Reports, 16(3), 437-014-0437-0. doi:10.1007/s11920-014-0437-0 [doi] 
Barnard-Brak, L., Davis, T. N., Schmidt, M., \& Richman, D. M. (2014). Effects associated with on- and off-label stimulant treatment of core autism and ADHD symptoms exhibited by children with autism spectrum disorder. Developmental Neurorehabilitation, doi:10.3109/17518423.2014.904949 [doi]

Bilder, D., Botts, E. L., Smith, K. R., Pimentel, R., Farley, M., Viskochil, J., . . Coon, H. (2013). Excess mortality and causes of death in autism spectrum disorders: A follow up of the 1980s utah/UCLA autism epidemiologic study. Journal of Autism and Developmental Disorders, 43(5), 1196-1204. doi:10.1007/s10803-012-1664-z [doi]

Billstedt, E., Gillberg, I. C., \& Gillberg, C. (2005). Autism after adolescence: Population-based 13- to 22-year follow-up study of 120 individuals with autism diagnosed in childhood. Journal of Autism and Developmental Disorders, 35(3), 351-360.

Billstedt, E., Gillberg, I. C., \& Gillberg, C. (2007). Autism in adults: Symptom patterns and early childhood predictors. use of the DISCO in a community sample followed from childhood. Journal of Child Psychology and Psychiatry, and Allied Disciplines, 48(11), 1102-1110. doi:JCPP1774 [pii]

Bitsko, R. H., Visser, S. N., Schieve, L. A., Ross, D. S., Thurman, D. J., \& Perou, R. (2009). Unmet health care needs among CSHCN with neurologic conditions. Pediatrics, 124 Suppl 4, S343-51. doi:10.1542/peds.20091255D; 10.1542/peds.2009-1255D

Blankenship, K., Erickson, C. A., Stigler, K. A., Posey, D. J., \& McDougle, C. J. (2010). Aripiprazole for irritability associated with autistic disorder in children and adolescents aged 6-17 years. Pediatric Health, 4(4), 375-381.

Broadstock, M., Doughty, C., \& Eggleston, M. (2007). Systematic review of the effectiveness of pharmacological treatments for adolescents and adults with autism spectrum disorder. Autism : The International Journal of Research and Practice, 11(4), 335-348. doi:11/4/335 [pii]

Bruder, M. B., Kerins, G., Mazzarella, C., Sims, J., \& Stein, N. (2012). Brief report: The medical care of adults with autism spectrum disorders: Identifying the needs. Journal of Autism and Developmental Disorders, 42(11), 2498-2504. doi:10.1007/s10803-012-1496-x [doi]

Brugha, T. S., Doos, L., Tempier, A., Einfeld, S., \& Howlin, P. (2015). Outcome measures in intervention trials for adults with autism spectrum disorders; a systematic review of assessments of core autism features and associated emotional and behavioural problems. International Journal of Methods in Psychiatric Research, 24(2), 99-115. doi:10.1002/mpr.1466 [doi]

Brugha, T. S., McManus, S., Bankart, J., Scott, F., Purdon, S., Smith, J., . . Meltzer, H. (2011). Epidemiology of autism spectrum disorders in adults in the community in england. Archives of General Psychiatry, 68(5), 459465. doi:10.1001/archgenpsychiatry.2011.38; 10.1001/archgenpsychiatry.2011.38

Buck, T. R., Viskochil, J., Farley, M., Coon, H., McMahon, W. M., Morgan, J., \& Bilder, D. A. (2014a). Psychiatric comorbidity and medication use in adults with autism spectrum disorder. Journal of Autism and Developmental Disorders, doi:10.1007/s10803-014-2170-2 [doi]

Buck, T. R., Viskochil, J., Farley, M., Coon, H., McMahon, W. M., Morgan, J., \& Bilder, D. A. (2014b). Psychiatric comorbidity and medication use in adults with autism spectrum disorder. Journal of Autism and Developmental Disorders, doi:10.1007/s10803-014-2170-2 [doi]

Buescher, A. V., Cidav, Z., Knapp, M., \& Mandell, D. S. (2014). Costs of autism spectrum disorders in the united kingdom and the united states. JAMA Pediatrics, 168(8), 721-728. doi:10.1001/jamapediatrics.2014.210 [doi] 
Buie, T., Campbell, D. B., Fuchs, G. J.,3rd, Furuta, G. T., Levy, J., Vandewater, J., . . Winter, H. (2010). Evaluation, diagnosis, and treatment of gastrointestinal disorders in individuals with ASDs: A consensus report. Pediatrics, 125 Suppl 1, S1-18. doi:10.1542/peds.2009-1878C [doi]

Bureau of Labor Statistics, US Department of Labor. (2014). Archived consumer price index detailed report information. Retrieved from http://www.bls.gov/cpi/cpi_dr.htm

Carolina-Nicole, H., John, H., \& Gregory, S. (2013). The impact of the mental health parity and addiction equity act on inpatient admissions . (Issue Brief No. 5). Washington D.C.: Healthcare Cost Institute.

Chambers, J. G., Kidron, Y., Spain, A. K., \& American Institutes, f. R. (2004). Characteristics of high-expenditure students with disabilities, 1999-2000. report 8. ().American Institutes for Research. Retrieved from http://search.ebscohost.com/login.aspx?direct=true \&db=eric\&AN=ED522071\&site=ehost-live

Ching, H., \& Pringsheim, T. (2012). Aripiprazole for autism spectrum disorders (ASD). The Cochrane Database of Systematic Reviews, 5, CD009043. doi:10.1002/14651858.CD009043.pub2; 10.1002/14651858.CD009043.pub2

Choudhry, L., Dougless, M., Lewis, J., Olson, C. H., Osterman, R., \& Shah, P. (2007). The impact of community health centers \& community-affiliated health plans on emergency department use. ().National Association of Community Health Centers; Association for Community Affiliated Plans.

Cidav, Z., Lawer, L., Marcus, S. C., \& Mandell, D. S. (2013). Age-related variation in health service use and associated expenditures among children with autism. Journal of Autism and Developmental Disorders, 43(4), 924-931. doi:10.1007/s10803-012-1637-2 [doi]

Cidav, Z., Marcus, S. C., \& Mandell, D. S. (2014). Home- and community-based waivers for children with autism: Effects on service use and costs. Intellectual and Developmental Disabilities, 52(4), 239-248. doi:10.1352/1934-9556-52.4.239 [doi]

Coury, D. L., Anagnostou, E., Manning-Courtney, P., Reynolds, A., Cole, L., McCoy, R., . . Perrin, J. M. (2012a). Use of psychotropic medication in children and adolescents with autism spectrum disorders. Pediatrics, 130 Suppl 2, S69-76. doi:10.1542/peds.2012-0900D; 10.1542/peds.2012-0900D

Coury, D. L., Anagnostou, E., Manning-Courtney, P., Reynolds, A., Cole, L., McCoy, R., . . Perrin, J. M. (2012b). Use of psychotropic medication in children and adolescents with autism spectrum disorders. Pediatrics, 130 Suppl 2, S69-76. doi:10.1542/peds.2012-0900D; 10.1542/peds.2012-0900D

Croen, L. A., Najjar, D. V., Ray, G. T., Lotspeich, L., \& Bernal, P. (2006). A comparison of health care utilization and costs of children with and without autism spectrum disorders in a large group-model health plan. Pediatrics, 118(4), e1203-11. doi:118/4/e1203 [pii]

Croen, L. A., Zerbo, O., Qian, Y., Massolo, M. L., Rich, S., Sidney, S., \& Kripke, C. (2015). The health status of adults on the autism spectrum. Autism: The International Journal of Research and Practice, doi:1362361315577517 [pii]

Cross-Disorder Group of the Psychiatric Genomics Consortium. (2013). Identification of risk loci with shared effects on five major psychiatric disorders: A genome-wide analysis. Lancet, 381(9875), 1371-1379. doi:10.1016/S0140-6736(12)62129-1 [doi]

Curran, G. M., Sullivan, G., Williams, K., Han, X., Collins, K., Keys, J., \& Kotrla, K. J. (2003). Emergency department use of persons with comorbid psychiatric and substance abuse disorders. Annals of Emergency Medicine, 41(5), 659-667. doi:10.1067/mem.2003.154 [doi] 
Curran, M. P. (2011). Aripiprazole in the treatment of irritability associated with autistic disorder in paediatric patients: Profile report. CNS Drugs, 25(9), 801-802. doi:10.2165/11208280-000000000-00000 [doi]

Dalsgaard, S., Nielsen, H. S., \& Simonsen, M. (2013). Five-fold increase in national prevalence rates of attentiondeficit/hyperactivity disorder medications for children and adolescents with autism spectrum disorder, attention-deficit/hyperactivity disorder, and other psychiatric disorders: A danish register-based study. Journal of Child and Adolescent Psychopharmacology, 23(7), 432-439. doi:10.1089/cap.2012.0111 [doi]

Developmental Disabilities Monitoring Network Surveillance Year 2010 Principal Investigators, \& Centers for Disease Control and Prevention (CDC). (2014). Prevalence of autism spectrum disorder among children aged 8 years - autism and developmental disabilities monitoring network, 11 sites, united states, 2010. Morbidity and Mortality Weekly Report.Surveillance Summaries (Washington, D.C.: 2002), 63(2), 1-21. doi:ss6302a1 [pii]

Doshi, A., Boudreaux, E. D., Wang, N., Pelletier, A. J., \& Camargo, C. A.,Jr. (2005). National study of US emergency department visits for attempted suicide and self-inflicted injury, 1997-2001. Annals of Emergency Medicine, 46(4), 369-375. doi:S0196-0644(05)00525-1 [pii]

Dove, D., Warren, Z., McPheeters, M. L., Taylor, J. L., Sathe, N. A., \& Veenstra-VanderWeele, J. (2012). Medications for adolescents and young adults with autism spectrum disorders: A systematic review. Pediatrics, 130(4), 717-726. doi:10.1542/peds.2012-0683; 10.1542/peds.2012-0683

Esbensen, A. J., Greenberg, J. S., Seltzer, M. M., \& Aman, M. G. (2009). A longitudinal investigation of psychotropic and non-psychotropic medication use among adolescents and adults with autism spectrum disorders. Journal of Autism and Developmental Disorders, 39(9), 1339-1349. doi:10.1007/s10803-009-07503 [doi]

Finlayson, J., Morrison, J., Jackson, A., Mantry, D., \& Cooper, S. A. (2010). Injuries, falls and accidents among adults with intellectual disabilities. prospective cohort study. Journal of Intellectual Disability Research : JIDR, 54(11), 966-980. doi:10.1111/j.1365-2788.2010.01319.x [doi]

Fombonne, E. (2003). Epidemiological surveys of autism and other pervasive developmental disorders: An update. Journal of Autism and Developmental Disorders, 33(4), 365-382.

Frazier, T. W., Shattuck, P. T., Narendorf, S. C., Cooper, B. P., Wagner, M., \& Spitznagel, E. L. (2011a). Prevalence and correlates of psychotropic medication use in adolescents with an autism spectrum disorder with and without caregiver-reported attention-deficit/hyperactivity disorder. Journal of Child and Adolescent Psychopharmacology, 21(6), 571-579. doi:10.1089/cap.2011.0057 [doi]

Frazier, T. W., Shattuck, P. T., Narendorf, S. C., Cooper, B. P., Wagner, M., \& Spitznagel, E. L. (2011b). Prevalence and correlates of psychotropic medication use in adolescents with an autism spectrum disorder with and without caregiver-reported attention-deficit/hyperactivity disorder. Journal of Child and Adolescent Psychopharmacology, 21(6), 571-579. doi:10.1089/cap.2011.0057; 10.1089/cap.2011.0057

Garcia-Villamisar, D., \& Rojahn, J. (2013). Comorbid psychopathology and stress mediate the relationship between autistic traits and repetitive behaviours in adults with autism. Journal of Intellectual Disability Research : $J I D R$, doi:10.1111/jir.12083 [doi]

Gerhard, T., Chavez, B., Olfson, M., \& Crystal, S. (2009). National patterns in the outpatient pharmacological management of children and adolescents with autism spectrum disorder. Journal of Clinical Psychopharmacology, 29(3), 307-310. doi:10.1097/JCP.0b013e3181a20c8a; 10.1097/JCP.0b013e3181a20c8a

Golnik, A., Ireland, M., \& Borowsky, I. W. (2009). Medical homes for children with autism: A physician survey. Pediatrics, 123(3), 966-971. doi:10.1542/peds.2008-1321 [doi] 
Gurney, J. G., McPheeters, M. L., \& Davis, M. M. (2006). Parental report of health conditions and health care use among children with and without autism: National survey of children's health. Archives of Pediatrics \& Adolescent Medicine, 160(8), 825-830. doi:10.1001/archpedi.160.8.825

Harris, S. L., \& Handleman, J. S. (1990). In Harris S. L., Handleman J. S. (Eds.), Aversive and nonaversive interventions: Controlling life-threatening behavior by the developmentally disabled. New York, NY US: Springer Publishing Co. Retrieved from http://search.ebscohost.com/login.aspx?direct=true \&db=psyh\&AN=1990-98360-000\&site=ehost-live

Healthcare Cost and Utilization Project (HCUP). (2011). HCUP nationwide emergency department sample (NEDS). (). Rockville, MD: Agency for Healthcare Research and Quality.

Heidgerken, A. D., Geffken, G., Modi, A., \& Frakey, L. (2005). A survey of autism knowledge in a health care setting. Journal of Autism and Developmental Disorders, 35(3), 323-330.

Hirota, T., Veenstra-Vanderweele, J., Hollander, E., \& Kishi, T. (2014). Antiepileptic medications in autism spectrum disorder: A systematic review and meta-analysis. Journal of Autism and Developmental Disorders, 44(4), 948-957. doi:10.1007/s10803-013-1952-2 [doi]

Hofvander, B., Delorme, R., Chaste, P., Nyden, A., Wentz, E., Stahlberg, O., . . Leboyer, M. (2009a). Psychiatric and psychosocial problems in adults with normal-intelligence autism spectrum disorders. BMC Psychiatry, 9, 35-244X-9-35. doi:10.1186/1471-244X-9-35 [doi]

Hofvander, B., Delorme, R., Chaste, P., Nyden, A., Wentz, E., Stahlberg, O., . . Leboyer, M. (2009b). Psychiatric and psychosocial problems in adults with normal-intelligence autism spectrum disorders. BMC Psychiatry, 9, 35-244X-9-35. doi:10.1186/1471-244X-9-35; 10.1186/1471-244X-9-35

Hollander, E., Wang, A. T., Braun, A., \& Marsh, L. (2009). Neurological considerations: Autism and parkinson's disease. Psychiatry Research, 170(1), 43-51. doi:10.1016/j.psychres.2008.07.014 [doi]

Horvath, K., Papadimitriou, J. C., Rabsztyn, A., Drachenberg, C., \& Tildon, J. T. (1999). Gastrointestinal abnormalities in children with autistic disorder. The Journal of Pediatrics, 135(5), 559-563. doi:S00223476(99)70052-1 [pii]

Howlin, P., Goode, S., Hutton, J., \& Rutter, M. (2004). Adult outcome for children with autism. Journal of Child Psychology and Psychiatry, and Allied Disciplines, 45(2), 212-229.

Hsieh, K., Heller, T., \& Miller, A. B. (2001). Risk factors for injuries and falls among adults with developmental disabilities. Journal of Intellectual Disability Research : JIDR, 45(Pt 1), 76-82. doi:jir277 [pii]

Imran, N., Chaudry, M. R., Azeem, M. W., Bhatti, M. R., Choudhary, Z. I., \& Cheema, M. A. (2011). A survey of autism knowledge and attitudes among the healthcare professionals in lahore, pakistan. BMC Pediatrics, 11 , 107-2431-11-107. doi:10.1186/1471-2431-11-107 [doi]

Jang, J., Dixon, D. R., Tarbox, J., \& Granpeesheh, D. (2011). Symptom severity and challenging behavior in children with ASD. Research in Autism Spectrum Disorders, 5(3), 1028-1032. Retrieved from http://search.ebscohost.com/login.aspx?direct=true \&db=eric\&AN=EJ917087\&site=ehost-live; http://dx.doi.org/10.1016/j.rasd.2010.11.008

Ji, N., \& Findling, R. L. (2015). An update on pharmacotherapy for autism spectrum disorder in children and adolescents. Current Opinion in Psychiatry, 28(2), 91-101. doi:10.1097/YCO.0000000000000132 [doi] 
Johnson, N., Frenn, M., Feetham, S., \& Simpson, P. (2011). Autism spectrum disorder: Parenting stress, family functioning and health-related quality of life. Families, Systems, \& Health, 29(3), 232-252.

doi: $10.1037 / \mathrm{a} 0025341$

Kalb, L. G., Stuart, E. A., Freedman, B., Zablotsky, B., \& Vasa, R. (2012). Psychiatric-related emergency department visits among children with an autism spectrum disorder. Pediatric Emergency Care, 28(12), 12691276. doi:10.1097/PEC.0b013e3182767d96 [doi]

Kamp-Becker, I., Schroder, J., Remschmidt, H., \& Bachmann, C. J. (2010). Health-related quality of life in adolescents and young adults with high functioning autism-spectrum disorder. Psycho-Social Medicine, 7 , 10.3205/psm000065. doi:10.3205/psm000065 [doi]

Kanner, L. (1943). Autistic disturbances of affective contact. Nervous Child, 2, 217-250. Retrieved from http://search.ebscohost.com/login.aspx?direct=true \&db=psyh\&AN=1943-03624-001\&site=ehost-live

Kato, K., Mikami, K., Akama, F., Yamada, K., Maehara, M., Kimoto, K., . . Matsumoto, H. (2013). Clinical features of suicide attempts in adults with autism spectrum disorders. General Hospital Psychiatry, 35(1), 5053. doi:10.1016/j.genhosppsych.2012.09.006 [doi]

Khanna, R., Jariwala, K., \& West-Strum, D. (2013). Use and cost of psychotropic drugs among recipients with autism in a state medicaid fee-for-service programme. Journal of Intellectual Disability Research : JIDR, 57(2), 161-171. doi:10.1111/j.1365-2788.2012.01563.x; 10.1111/j.1365-2788.2012.01563.x

Khanna, R., Madhavan, S. S., Smith, M. J., Patrick, J. H., Tworek, C., \& Becker-Cottrill, B. (2011). Assessment of health-related quality of life among primary caregivers of children with autism spectrum disorders. Journal of Autism and Developmental Disorders, 41(9), 1214-1227. Retrieved from http://search.ebscohost.com/login.aspx?direct=true\&db=eric\&AN=EJ936187\&site=ehost-live; http://dx.doi.org/10.1007/s10803-010-1140-6

Knapp, M., Romeo, R., \& Beecham, J. (2009). Economic cost of autism in the UK. Autism : The International Journal of Research and Practice, 13(3), 317-336. doi:10.1177/1362361309104246 [doi]

Kogan, M. D., Strickland, B. B., Blumberg, S. J., Singh, G. K., Perrin, J. M., \& van Dyck, P. C. (2008). A national profile of the health care experiences and family impact of autism spectrum disorder among children in the united states, 2005-2006. Pediatrics, 122(6), e1149-58. doi:10.1542/peds.2008-1057; 10.1542/peds.2008-1057

Kohane, I. S., McMurry, A., Weber, G., MacFadden, D., Rappaport, L., Kunkel, L., . . Churchill, S. (2012). The co-morbidity burden of children and young adults with autism spectrum disorders. PloS One, 7(4), e33224. doi:10.1371/journal.pone.0033224; 10.1371/journal.pone.0033224

Kraijer, D. (1999). Autism and autistic-like conditions in mental retardation. Journal of Intellectual Disability Research, 43(4), 341. Retrieved from http://search.ebscohost.com/login.aspx?direct=true \&db=a9h\&AN=5183802\&site=ehost-live

La Malfa, G., Lassi, S., Bertelli, M., Salvini, R., \& Placidi, G. F. (2004). Autism and intellectual disability: A study of prevalence on a sample of the italian population. Journal of Intellectual Disability Research, 48(3), 262267. doi:10.1111/j.1365-2788.2003.00567.x

Lake, J. K., Balogh, R., \& Lunsky, Y. (2012). Polypharmacy profiles and predictors among adults with autism spectrum disorders. Research in Autism Spectrum Disorders, 6(3), 1142-1149. 
Lazoff, T., Zhong, L., Piperni, T., \& Fombonne, E. (2010). Prevalence of pervasive developmental disorders among children at the english montreal school board. Canadian Journal of Psychiatry.Revue Canadienne De Psychiatrie, 55(11), 715-720.

LeClerc, S., \& Easley, D. (2015). Pharmacological therapies for autism spectrum disorder: A review. $P$ \& $T$ : A Peer-Reviewed Journal for Formulary Management, 40(6), 389-397.

Leslie, D. L., \& Martin, A. (2007). Health care expenditures associated with autism spectrum disorders. Archives of Pediatrics \& Adolescent Medicine, 161(4), 350-355. doi:10.1001/archpedi.161.4.350

Levy, S. E., Giarelli, E., Lee, L. C., Schieve, L. A., Kirby, R. S., Cunniff, C., . . Rice, C. E. (2010). Autism spectrum disorder and co-occurring developmental, psychiatric, and medical conditions among children in multiple populations of the united states. Journal of Developmental and Behavioral Pediatrics : JDBP, 31(4), 267-275. doi:10.1097/DBP.0b013e3181d5d03b; 10.1097/DBP.0b013e3181d5d03b

Leyfer, O. T., Folstein, S. E., Bacalman, S., Davis, N. O., Dinh, E., Morgan, J., . . Lainhart, J. E. (2006). Comorbid psychiatric disorders in children with autism: Interview development and rates of disorders. Journal of Autism and Developmental Disorders, 36(7), 849-861. doi:10.1007/s10803-006-0123-0 [doi]

Liptak, G. S., Orlando, M., Yingling, J. T., Theurer-Kaufman, K. L., Malay, D. P., Tompkins, L. A., \& Flynn, J. R. (2006). Satisfaction with primary health care received by families of children with developmental disabilities. Journal of Pediatric Health Care : Official Publication of National Association of Pediatric Nurse Associates \& Practitioners, 20(4), 245-252. doi:10.1016/j.pedhc.2005.12.008

Liptak, G. S., Stuart, T., \& Auinger, P. (2006). Health care utilization and expenditures for children with autism: Data from U.S. national samples. Journal of Autism and Developmental Disorders, 36(7), 871-879. doi:10.1007/s10803-006-0119-9

Logan, S. L., Nicholas, J. S., Carpenter, L. A., King, L. B., Garrett-Mayer, E., \& Charles, J. M. (2012). High prescription drug use and associated costs among medicaid-eligible children with autism spectrum disorders identified by a population-based surveillance network. Annals of Epidemiology, 22(1), 1-8. doi:10.1016/j.annepidem.2011.10.007; 10.1016/j.annepidem.2011.10.007

Lokhandwala, T., Khanna, R., \& West-Strum, D. (2012). Hospitalization burden among individuals with autism. Journal of Autism and Developmental Disorders, 42(1), 95-104. doi:10.1007/s10803-011-1217-x [doi]

Lugnegard, T., Hallerback, M. U., \& Gillberg, C. (2011a). Psychiatric comorbidity in young adults with a clinical diagnosis of asperger syndrome. Research in Developmental Disabilities, 32(5), 1910-1917. doi:10.1016/j.ridd.2011.03.025 [doi]

Lugnegard, T., Hallerback, M. U., \& Gillberg, C. (2011b). Psychiatric comorbidity in young adults with a clinical diagnosis of asperger syndrome. Research in Developmental Disabilities, 32(5), 1910-1917. doi:10.1016/j.ridd.2011.03.025; 10.1016/j.ridd.2011.03.025

Lunsky, Y., Gracey, C., \& Bradley, E. (2009). Adults with autism spectrum disorders using psychiatric hospitals in ontario: Clinical profile and service needs. Research in Autism Spectrum Disorders, 3(4), 1006-1013.

Magiati, I., Tay, X. W., \& Howlin, P. (2014). Cognitive, language, social and behavioural outcomes in adults with autism spectrum disorders: A systematic review of longitudinal follow-up studies in adulthood. Clinical Psychology Review, 34(1), 73-86. doi:10.1016/j.cpr.2013.11.002 [doi]

Mandell, D. S. (2008a). Psychiatric hospitalization among children with autism spectrum disorders. Journal of Autism and Developmental Disorders, 38(6), 1059-1065. doi:10.1007/s10803-007-0481-2 [doi] 
Mandell, D. S. (2008b). Psychiatric hospitalization among children with autism spectrum disorders. Journal of Autism and Developmental Disorders, 38(6), 1059-1065. doi:10.1007/s10803-007-0481-2 [doi]

Mandell, D. S., Cao, J., Ittenbach, R., \& Pinto-Martin, J. (2006). Medicaid expenditures for children with autistic spectrum disorders: 1994 to 1999. Journal of Autism and Developmental Disorders, 36(4), 475-485. doi:10.1007/s10803-006-0088-z

Mandell, D. S., Morales, K. H., Marcus, S. C., Stahmer, A. C., Doshi, J., \& Polsky, D. E. (2008a). Psychotropic medication use among medicaid-enrolled children with autism spectrum disorders. Pediatrics, 121(3), e441-8. doi:10.1542/peds.2007-0984; 10.1542/peds.2007-0984

Mandell, D. S., Morales, K. H., Marcus, S. C., Stahmer, A. C., Doshi, J., \& Polsky, D. E. (2008b). Psychotropic medication use among medicaid-enrolled children with autism spectrum disorders. Pediatrics, 121(3), e441-8. doi:10.1542/peds.2007-0984; 10.1542/peds.2007-0984

Marcus, R. N., Owen, R., Kamen, L., Manos, G., McQuade, R. D., Carson, W. H., \& Aman, M. G. (2009). A placebo-controlled, fixed-dose study of aripiprazole in children and adolescents with irritability associated with autistic disorder. Journal of the American Academy of Child and Adolescent Psychiatry, 48(11), 11101119. doi:10.1097/CHI.0b013e3181b76658 [doi]

Marriage, S., Wolverton, A., \& Marriage, K. (2009). Autism spectrum disorder grown up: A chart review of adult functioning. Journal of the Canadian Academy of Child and Adolescent Psychiatry $=$ Journal De L'Academie Canadienne De Psychiatrie De L'Enfant Et De L'Adolescent, 18(4), 322-328.

Maski, K. P., Jeste, S. S., \& Spence, S. J. (2011). Common neurological co-morbidities in autism spectrum disorders. Current Opinion in Pediatrics, 23(6), 609-615. doi:10.1097/MOP.0b013e32834c9282; 10.1097/MOP.0b013e32834c9282

Matson, J. L., \& Rivet, T. T. (2008). Characteristics of challenging behaviours in adults with autistic disorder, PDDNOS, and intellectual disability. Journal of Intellectual \& Developmental Disability, 33(4), 323-329. doi:10.1080/13668250802492600; 10.1080/13668250802492600

Matson, J. L., Sipes, M., Fodstad, J. C., \& Fitzgerald, M. E. (2011). Issues in the management of challenging behaviours of adults with autism spectrum disorder. CNS Drugs, 25(7), 597-606. doi:10.2165/11591700000000000-00000 [doi]

Matson, J. L., Wilkins, J., \& Macken, J. (2009). The relationship of challenging behaviors to severity and symptoms of autism spectrum disorders. Journal of Mental Health Research in Intellectual Disabilities, 2(1), 29-44. Retrieved from http://search.ebscohost.com/login.aspx?direct=true \&db=eric\&AN=EJ866814\&site=ehost-live; http://www.informaworld.com/openurl?genre=article\&id=doi:10.1080/19315860802611415

Mauch, D., Pfefferle, S., Booker, C., Pustell, M., \& Levin, J. (2011). Report on state services to individuals with autism spectrum disorders (ASD). ( No. S-10 CMS-33 No. 2). Centers for Medicare \& Medicaid Services (CMS) ASD Services Project: Centers for Medicare \& Medicaid Services (CMS).

McCracken, J. T., McGough, J., Shah, B., Cronin, P., Hong, D., Aman, M. G., . . Research Units on Pediatric Psychopharmacology Autism Network. (2002). Risperidone in children with autism and serious behavioral problems. The New England Journal of Medicine, 347(5), 314-321. doi:10.1056/NEJMoa013171

McElhanon, B. O., McCracken, C., Karpen, S., \& Sharp, W. G. (2014). Gastrointestinal symptoms in autism spectrum disorder: A meta-analysis. Pediatrics, doi:peds.2013-3995 [pii] 
MESIBOV, G. B., \& HANDLAN, S. (1997). In Cohen H. J., Volkmar F. R. (Eds.), Handbook of autism and pervasive developmental disorders. New York: Wiley.

Miller, S. M. (2015). Nurses caring for adults with autism in an emergency department: A survey of knowledge. Doctoral Projects, (7)

Morgan, C. N., Roy, M., Nasr, A., Chance, P., Hand, M., Mlele, T., \& Roy, A. (2002). A community survey establishing the prevalence rate of autistic disorder in adults with learning disability. Psychiatric Bulletin, 26(4), 127-130. doi:10.1192/pb.26.4.127

Mouridsen, S. E., Bronnum-Hansen, H., Rich, B., \& Isager, T. (2008). Mortality and causes of death in autism spectrum disorders: An update. Autism : The International Journal of Research and Practice, 12(4), 403-414. doi:10.1177/1362361308091653 [doi]

Mukaetova-Ladinska, E. B., Perry, E., Baron, M., Povey, C., \& Autism Ageing Writing Group. (2012a). Ageing in people with autistic spectrum disorder. International Journal of Geriatric Psychiatry, 27(2), 109-118. doi:10.1002/gps.2711; 10.1002/gps.2711

Mukaetova-Ladinska, E. B., Perry, E., Baron, M., Povey, C., \& Autism Ageing Writing Group. (2012b). Ageing in people with autistic spectrum disorder. International Journal of Geriatric Psychiatry, 27(2), 109-118. doi:10.1002/gps.2711; 10.1002/gps.2711

Murphy, G. H., Beadle-Brown, J., Wing, L., Gould, J., Shah, A., \& Holmes, N. (2005). Chronicity of challenging behaviours in people with severe intellectual disabilities and/or autism: A total population sample. Journal of Autism \& Developmental Disorders, 35(4), 405-418. doi:10.1007/s10803-005-5030-2

Myer, P. A., Mannalithara, A., Singh, G., Singh, G., Pasricha, P. J., \& Ladabaum, U. (2013). Clinical and economic burden of emergency department visits due to gastrointestinal diseases in the united states. The American Journal of Gastroenterology, 108(9), 1496-1507. doi:10.1038/ajg.2013.199 [doi]

Myers, S. M. (2007). The status of pharmacotherapy for autism spectrum disorders. Expert Opinion on Pharmacotherapy, 8(11), 1579-1603. doi:10.1517/14656566.8.11.1579 [doi]

National Committee for Quality Assurance. (2015). HEDIS and quality measurement. HEDIS 2015 final NDC lists. Retrieved from http://www.ncqa.org/HEDISQualityMeasurement/HEDISMeasures/HEDIS2015/HEDIS2015NDCLicense/HE DIS2015FinalNDCLists.aspx

National Conference of State Legislatures. (2014). Recent medicaid prescription drug laws and strategies. Retrieved from http://www.ncsl.org/research/health/medicaid-pharmaceutical-laws-and-policies.aspx

Nicolaidis, C., Raymaker, D., McDonald, K., Dern, S., Boisclair, W. C., Ashkenazy, E., \& Baggs, A. (2013). Comparison of healthcare experiences in autistic and non-autistic adults: A cross-sectional online survey facilitated by an academic-community partnership. Journal of General Internal Medicine, 28(6), 761-769. doi:10.1007/s11606-012-2262-7 [doi]

Oskoui, M., \& Wolfson, C. (2012). Treatment comfort of adult neurologists in childhood onset conditions. The Canadian Journal of Neurological Sciences.Le Journal Canadien Des Sciences Neurologiques, 39(2), 202205. doi:HM223822024126U6 [pii]

Oswald, D. P., \& Sonenklar, N. A. (2007a). Medication use among children with autism spectrum disorders. Journal of Child and Adolescent Psychopharmacology, 17(3), 348-355. doi:10.1089/cap.2006.17303 
Oswald, D. P., \& Sonenklar, N. A. (2007b). Medication use among children with autism spectrum disorders. Journal of Child and Adolescent Psychopharmacology, 17(3), 348-355. doi:10.1089/cap.2006.17303 [doi]

Owen, R., Sikich, L., Marcus, R. N., Corey-Lisle, P., Manos, G., McQuade, R. D., . . Findling, R. L. (2009). Aripiprazole in the treatment of irritability in children and adolescents with autistic disorder. Pediatrics, 124(6), 1533-1540. doi:10.1542/peds.2008-3782 [doi]

Owens, P. L., Barrett, M. L., Gibson, T. B., Andrews, R. M., Weinick, R. M., \& Mutter, R. L. (2010). Emergency department care in the united states: A profile of national data sources. Annals of Emergency Medicine, 56(2), 150-165. doi:10.1016/j.annemergmed.2009.11.022 [doi]

Palucka, A. M., \& Lunsky, Y. (2007). Review of inpatient admissions of individuals with autism spectrum disorders to a specialized dual diagnosis program. Journal on Developmental Disabilities, 13(1), 205-209.

Peacock, G., Amendah, D., Ouyang, L., \& Grosse, S. D. (2012). Autism spectrum disorders and health care expenditures: The effects of co-occurring conditions. Journal of Developmental and Behavioral Pediatrics : JDBP, 33(1), 2-8. doi:10.1097/DBP.0b013e31823969de; 10.1097/DBP.0b013e31823969de

Pellicano, E., Dinsmore, A., \& Charman, T. (2014). What should autism research focus upon? community views and priorities from the united kingdom. Autism : The International Journal of Research and Practice, 18(7), 756770. doi:10.1177/1362361314529627 [doi]

Pines, J. M., Asplin, B. R., Kaji, A. H., Lowe, R. A., Magid, D. J., Raven, M., . . Yealy, D. M. (2011). Frequent users of emergency department services: Gaps in knowledge and a proposed research agenda. Academic Emergency Medicine : Official Journal of the Society for Academic Emergency Medicine, 18(6), e64-9. doi:10.1111/j.1553-2712.2011.01086.x [doi]

Piven, J., Rabins, P., \& Autism-in-Older Adults Working Group. (2011). Autism spectrum disorders in older adults: Toward defining a research agenda. Journal of the American Geriatrics Society, 59(11), 2151-2155. doi:10.1111/j.1532-5415.2011.03632.x [doi]

Quality Resource Systems, I. (2006). Area resource file. (). Fairfax, VA: Health Resources and Services Administration, US Department of Health and Human Services, 2006.

Reichow, B. (2012). Overview of meta-analyses on early intensive behavioral intervention for young children with autism spectrum disorders. Journal of Autism and Developmental Disorders, 42(4), 512-520. doi:10.1007/s10803-011-1218-9; 10.1007/s10803-011-1218-9

Richmond, T. S., Hollander, J. E., Ackerson, T. H., Robinson, K., Gracias, V., Shults, J., \& Amsterdam, J. (2007). Psychiatric disorders in patients presenting to the emergency department for minor injury. Nursing Research, 56(4), 275-282. doi:10.1097/01.NNR.0000280616.13566.84 [doi]

Rojo, L. E., Gaspar, P. A., Silva, H., Risco, L., Arena, P., Cubillos-Robles, K., \& Jara, B. (2015). Metabolic syndrome and obesity among users of second generation antipsychotics: A global challenge for modern psychopharmacology. Pharmacological Research : The Official Journal of the Italian Pharmacological Society, doi:S1043-6618(15)00155-3 [pii]

Rosenberg, R. E., Mandell, D. S., Farmer, J. E., Law, J. K., Marvin, A. R., \& Law, P. A. (2010a). Psychotropic medication use among children with autism spectrum disorders enrolled in a national registry, 2007-2008. Journal of Autism and Developmental Disorders, 40(3), 342-351. doi:10.1007/s10803-009-0878-1; 10.1007/s10803-009-0878-1 
Rosenberg, R. E., Mandell, D. S., Farmer, J. E., Law, J. K., Marvin, A. R., \& Law, P. A. (2010b). Psychotropic medication use among children with autism spectrum disorders enrolled in a national registry, 2007-2008. Journal of Autism and Developmental Disorders, 40(3), 342-351. doi:10.1007/s10803-009-0878-1; 10.1007/s10803-009-0878-1

Rubenstein, E., Wiggins, L. D., \& Lee, L. C. (2015). A review of the differences in developmental, psychiatric, and medical endophenotypes between males and females with autism spectrum disorder. Journal of Developmental and Physical Disabilities, 27(1), 119-139. doi:10.1007/s10882-014-9397-x [doi]

Ruble, L. A., Heflinger, C. A., Renfrew, J. W., \& Saunders, R. C. (2005). Access and service use by children with autism spectrum disorders in medicaid managed care. Journal of Autism and Developmental Disorders, 35(1), 3-13.

Rumsey, J. M., Rapoport, J. L., \& Sceery, W. R. (1985). Autistic children as adults: Psychiatric, social, and behavioral outcomes. Journal of the American Academy of Child Psychiatry, 24(4), 465-473.

Santosh, P. J., \& Mijovic, A. (2006). Does pervasive developmental disorder protect children and adolescents against drug and alcohol use? European Child \& Adolescent Psychiatry, 15(4), 183-188. doi:10.1007/s00787005-0517-0 [doi]

Schieve, L. A., Gonzalez, V., Boulet, S. L., Visser, S. N., Rice, C. E., Van Naarden Braun, K., \& Boyle, C. A. (2012). Concurrent medical conditions and health care use and needs among children with learning and behavioral developmental disabilities, national health interview survey, 2006-2010. Research in Developmental Disabilities, 33(2), 467-476. doi:10.1016/j.ridd.2011.10.008 [doi]

Schubart, J. R., Camacho, F., \& Leslie, D. (2014). Psychotropic medication trends among children and adolescents with autism spectrum disorder in the medicaid program. Autism : The International Journal of Research and Practice, 18(6), 631-637. doi:10.1177/1362361313497537 [doi]

Seltzer, M. M., Krauss, M. W., Shattuck, P. T., Orsmond, G., Swe, A., \& Lord, C. (2003). The symptoms of autism spectrum disorders in adolescence and adulthood. Journal of Autism and Developmental Disorders, 33(6), 565-581.

Semansky, R. M., Xie, M., \& Mandell, D. S. (2011a). Medicaid's increasing role in treating youths with autism spectrum disorders. Psychiatric Services (Washington, D.C.), 62(6), 588. doi:10.1176/appi.ps.62.6.588; 10.1176/appi.ps.62.6.588

Semansky, R. M., Xie, M., \& Mandell, D. S. (2011b). Medicaid's increasing role in treating youths with autism spectrum disorders. Psychiatric Services (Washington, D.C.), 62(6), 588-588. Retrieved from http://search.ebscohost.com/login.aspx?direct=true $\& \mathrm{db}=\mathrm{cmedm} \& A N=21632723 \&$ site=ehost-live

Shattuck, P. T., Roux, A. M., Hudson, L. E., Taylor, J. L., Maenner, M. J., \& Trani, J. F. (2012). Services for adults with an autism spectrum disorder. Canadian Journal of Psychiatry.Revue Canadienne De Psychiatrie, 57(5), 284-291.

Shimabukuro, T. T., Grosse, S. D., \& Rice, C. (2008). Medical expenditures for children with an autism spectrum disorder in a privately insured population. Journal of Autism and Developmental Disorders, 38(3), 546-552. doi:10.1007/s10803-007-0424-y

Siklos, S., \& Kerns, K. A. (2006). Assessing need for social support in parents of children with autism and down syndrome. Journal of Autism and Developmental Disorders, 36(7), 921-933. doi:10.1007/s10803-006-0129-7 
Sills, M. R., \& Bland, S. D. (2002). Summary statistics for pediatric psychiatric visits to US emergency departments, 1993-1999. Pediatrics, 110(4), e40.

Simonoff, E., Pickles, A., Charman, T., Chandler, S., Loucas, T., \& Baird, G. (2008). Psychiatric disorders in children with autism spectrum disorders: Prevalence, comorbidity, and associated factors in a populationderived sample. Journal of the American Academy of Child and Adolescent Psychiatry, 47(8), 921-929. doi:10.1097/CHI.0b013e318179964f [doi]

Sizoo, B., van den Brink, W., Gorissen van Eenige, M., \& van der Gaag, R. J. (2009). Personality characteristics of adults with autism spectrum disorders or attention deficit hyperactivity disorder with and without substance use disorders. The Journal of Nervous and Mental Disease, 197(6), 450-454. doi:10.1097/NMD.0b013e3181a61dd0 [doi]

Smith, M. W., Stocks, C., \& Santora, P. B. (2015). Hospital readmission rates and emergency department visits for mental health and substance abuse conditions. Community Mental Health Journal, 51(2), 190-197. doi:10.1007/s10597-014-9784-x [doi]

Soto, E. C., Frederickson, A. M., Trivedi, H., Le, A., Eugene, M. C., Shekher, M., . . Correll, C. U. (2009). Frequency and correlates of inappropriate pediatric psychiatric emergency room visits. The Journal of Clinical Psychiatry, 70(8), 1164-1177. doi:10.4088/JCP.08m04839 [doi]

Spencer, D., Marshall, J., Post, B., Kulakodlu, M., Newschaffer, C., Dennen, T., . . Jain, A. (2013). Psychotropic medication use and polypharmacy in children with autism spectrum disorders. Pediatrics, 132(5), 833-840. doi:10.1542/peds.2012-3774; 10.1542/peds.2012-3774

Starkstein, S., Gellar, S., Parlier, M., Payne, L., \& Piven, J. (2015). High rates of parkinsonism in adults with autism. Journal of Neurodevelopmental Disorders, 7(1), 29-015-9125-6. Epub 2015 Aug 30. doi:10.1186/s11689-015-9125-6 [doi]

Tang, N., Stein, J., Hsia, R. Y., Maselli, J. H., \& Gonzales, R. (2010). Trends and characteristics of US emergency department visits, 1997-2007. JAMA : The Journal of the American Medical Association, 304(6), 664-670. doi:10.1001/jama.2010.1112 [doi]

The Henry J Kaiser Family Foundation. (2013). State HMO penetration rate, state health facts. Retrieved from http://kff.org/other/state-indicator/hmo-penetration-rate/

Tonge, B., \& Brereton, A. (2011). Autism spectrum disorders. Australian Family Physician, 40(9), 672-677. Retrieved from http://search.ebscohost.com/login.aspx?direct=true $\& d b=c m e d m \& A N=21894273 \&$ site=ehostlive

Tsakanikos, E., Costello, H., Holt, G., Sturmey, P., \& Bouras, N. (2007). Behaviour management problems as predictors of psychotropic medication and use of psychiatric services in adults with autism. Journal of Autism and Developmental Disorders, 37(6), 1080-1085. doi:10.1007/s10803-006-0248-1 [doi]

Unenge Hallerback, M., Lugnegard, T., \& Gillberg, C. (2012). Is autism spectrum disorder common in schizophrenia? Psychiatry Research, 198(1), 12-17. doi:10.1016/j.psychres.2012.01.016; 10.1016/j.psychres.2012.01.016

van Niekerk, M. E., Groen, W., Vissers, C. T., van Driel-de Jong, D., Kan, C. C., \& Oude Voshaar, R. C. (2011). Diagnosing autism spectrum disorders in elderly people. International Psychogeriatrics / IPA, 23(5), 700-710. doi:10.1017/S1041610210002152; 10.1017/S1041610210002152

van Rooijen, M. (2005). Autistische oudere lijkt niet te bestaan. Psy, 6, 4-5. 
van Steensel, F. J., Bogels, S. M., \& de Bruin, E. I. (2013). Psychiatric comorbidity in children with autism spectrum disorders: A comparison with children with ADHD. Journal of Child and Family Studies, 22(3), 368-376. doi:10.1007/s10826-012-9587-z [doi]

Vanbergeijk, E., Klin, A., \& Volkmar, F. (2008). Supporting more able students on the autism spectrum: College and beyond. Journal of Autism and Developmental Disorders, 38(7), 1359-1370. doi:10.1007/s10803-0070524-8 [doi]

Velott, D. L., Agbese, E., Mandell, D., Stein, B. D., Dick, A. W., Yu, H., \& Leslie, D. L. (2015). Medicaid 1915(c) home- and community-based services waivers for children with autism spectrum disorder. Autism : The International Journal of Research and Practice, doi:1362361315590806 [pii]

Volkmar, F., Cook, E. H.,Jr, Pomeroy, J., Realmuto, G., \& Tanguay, P. (1999). Practice parameters for the assessment and treatment of children, adolescents, and adults with autism and other pervasive developmental disorders. american academy of child and adolescent psychiatry working group on quality issues. Journal of the American Academy of Child and Adolescent Psychiatry, 38(12 Suppl), 32S-54S.

Wang, L., \& Leslie, D. L. (2010a). Health care expenditures for children with autism spectrum disorders in medicaid. Journal of the American Academy of Child and Adolescent Psychiatry, 49(11), 1165-1171. doi:10.1016/j.jaac.2010.08.003; 10.1016/j.jaac.2010.08.003

Wang, L., \& Leslie, D. L. (2010b). Health care expenditures for children with autism spectrum disorders in medicaid. Journal of the American Academy of Child and Adolescent Psychiatry, 49(11), 1165-1171. doi:10.1016/j.jaac.2010.08.003; 10.1016/j.jaac.2010.08.003

Wang, L., Mandell, D. S., Lawer, L., Cidav, Z., \& Leslie, D. L. (2012). Healthcare service use and costs for autism spectrum disorder: A comparison between medicaid and private insurance. Journal of Autism and Developmental Disorders, doi:10.1007/s10803-012-1649-y

Warren, Z., Veenstra-VanderWeele, J., Stone, W., Bruzek, J. L., Nahmias, A. S., Foss-Feig, J. H., ... McPheeters, M. L. (April 2011). Therapies for children with autism spectrum disorders.. (Comparative Effectiveness Reviews no. 26 No. 11-EHC029-EF). Rockville (MD): Agency for Healthcare Research and Quality. doi:http://effectivehealthcare.ahrq.gov/ehc/products/106/651/Autism_Disorder_exec-summ.pdf

West, L., Brunssen, S. H., \& Waldrop, J. (2009). Review of the evidence for treatment of children with autism with selective serotonin reuptake inhibitors. Journal for Specialists in Pediatric Nursing : JSPN, 14(3), 183-191. doi:10.1111/j.1744-6155.2009.00196.x [doi]

Wharff, E. A., Ginnis, K. B., Ross, A. M., \& Blood, E. A. (2011). Predictors of psychiatric boarding in the pediatric emergency department: Implications for emergency care. Pediatric Emergency Care, 27(6), 483-489. doi:10.1097/PEC.0b013e31821d8571 [doi]

White, S. W., Oswald, D., Ollendick, T., \& Scahill, L. (2009). Anxiety in children and adolescents with autism spectrum disorders. Clinical Psychology Review, 29(3), 216-229. doi:10.1016/j.cpr.2009.01.003; 10.1016/j.cpr.2009.01.003

Williams, E. R., Guthrie, E., Mackway-Jones, K., James, M., Tomenson, B., Eastham, J., \& McNally, D. (2001). Psychiatric status, somatisation, and health care utilization of frequent attenders at the emergency department: A comparison with routine attenders. Journal of Psychosomatic Research, 50(3), 161-167. doi:S00223999(00)00228-2 [pii] 
Williams, K., Wheeler, D. M., Silove, N., \& Hazell, P. (2010). Selective serotonin reuptake inhibitors (SSRIs) for autism spectrum disorders (ASD). The Cochrane Database of Systematic Reviews, (8):CD004677. doi(8), CD004677. doi:10.1002/14651858.CD004677.pub2; 10.1002/14651858.CD004677.pub2

Zafeiriou, D. I., Ververi, A., \& Vargiami, E. (2007). Childhood autism and associated comorbidities. Brain \& Development, 29(5), 257-272. doi:S0387-7604(06)00209-9 [pii]

Zito, J. M., Derivan, A. T., Kratochvil, C. J., Safer, D. J., Fegert, J. M., \& Greenhill, L. L. (2008). Off-label psychopharmacologic prescribing for children: History supports close clinical monitoring. Child and Adolescent Psychiatry and Mental Health, 2(1), 24. doi:10.1186/1753-2000-2-24 [doi] 


\title{
Study 1. The Burden of Comorbidities, Healthcare Utilization, and Expenditures in Adult Fee-for-Service Medicaid Enrollees with and without Autism Spectrum Disorders (ASD)
}

\author{
Prepared by Rini Vohra
}

For Submission to PhD Dissertation Committee Members

Dr. Suresh Madhavan

Dr. Usha Sambamoorthi

Dr. Nilanjana Dwibedi

Dr. Claire StPeter

Dr. Susannah Poe 


\section{PhD Dissertation Chapter 2}

\section{CONTENTS}

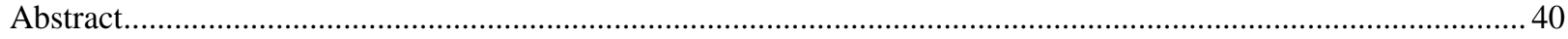

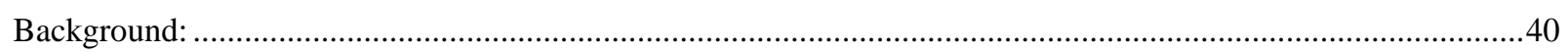

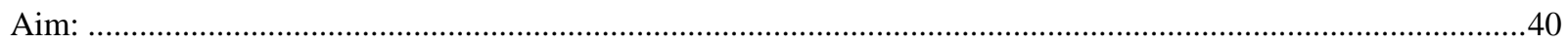

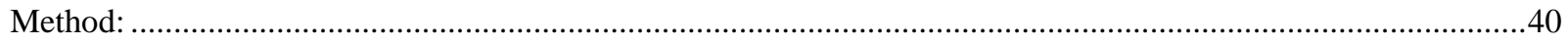

Results:

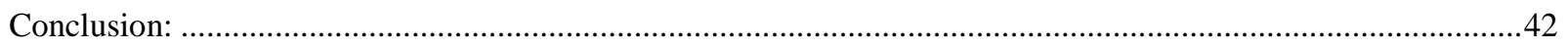

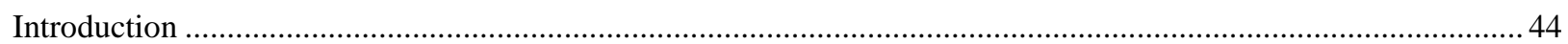

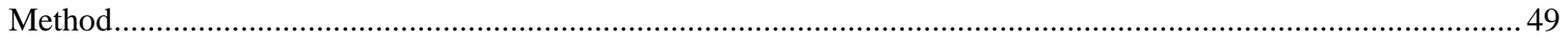

Study Population and Design ...............................................................................................................

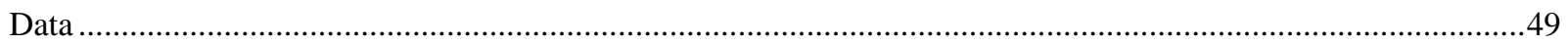

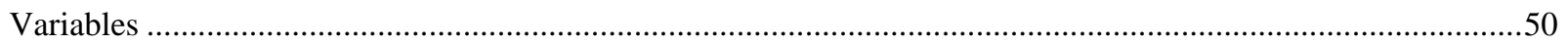

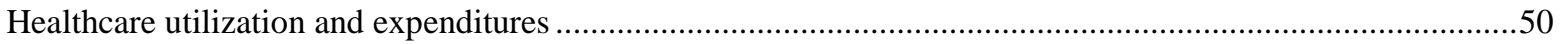

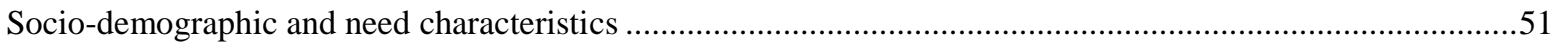

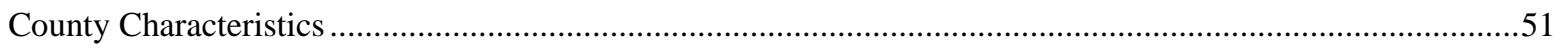

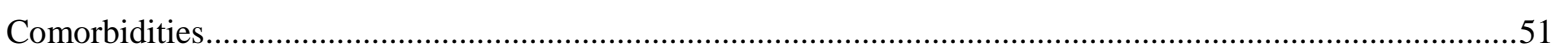

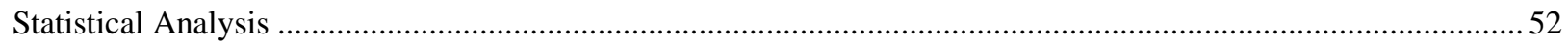

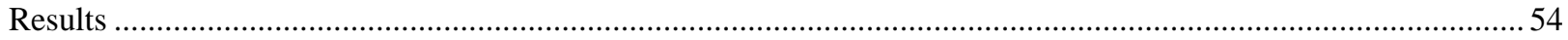

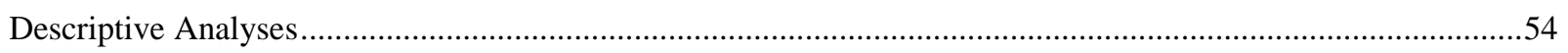

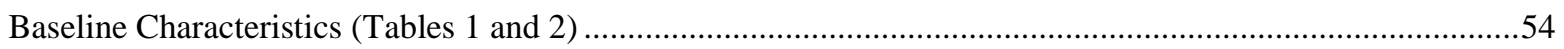

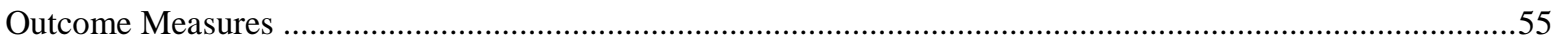

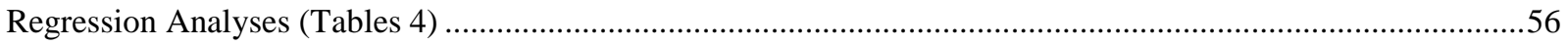

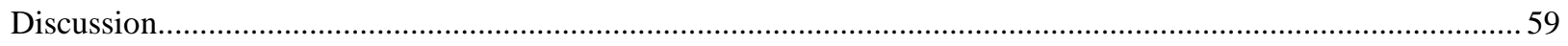

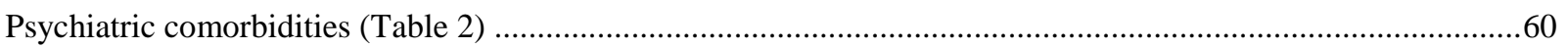

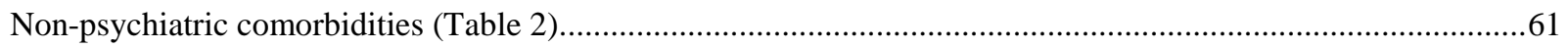

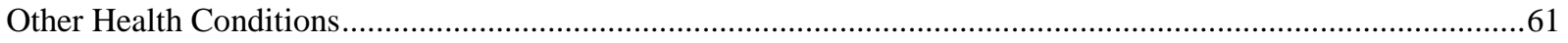

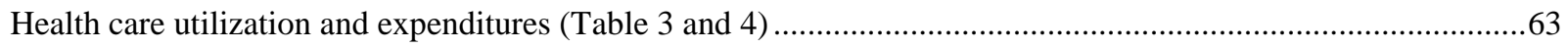

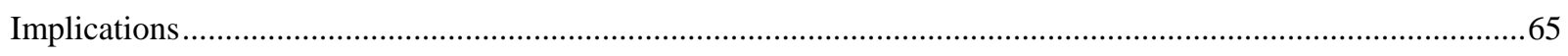

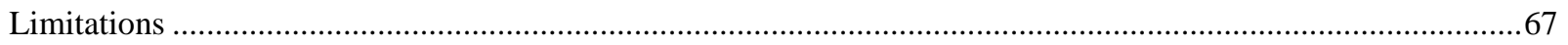

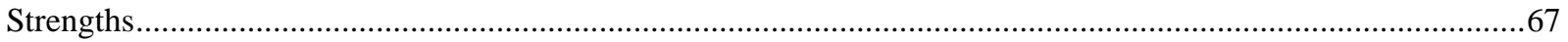

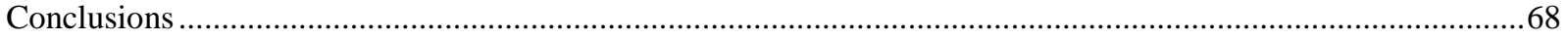

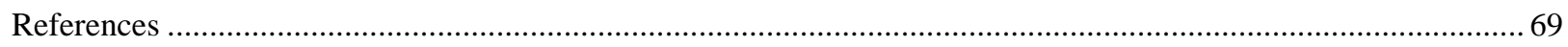

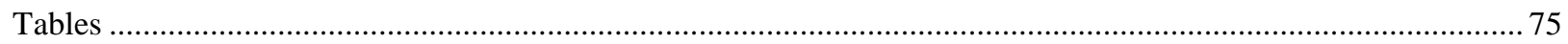


PhD Dissertation Chapter 2

\begin{abstract}
Background: Individuals with ASD have extensive comorbidities which affect their health related quality of life. The current literature lacks documentation of prevalence of different types of comorbidities and their association with healthcare utilization and expenditures among adults with ASD as compared to adults without ASD.
\end{abstract}

Aim: To determine: 1) the differences in prevalence of psychiatric, non-psychiatric comorbidities, other health conditions, healthcare utilization, and expenditures among adults with and without ASD; and 2) the association of type of comorbidity with healthcare utilization and expenditures of adults with ASD.

Method: A retrospective cohort study using claims data from three state Medicaid Analytic eXtract was conducted. Adults aged 22-64 years with ASD (ICD9-CM code: 299.xx) with at least one inpatient or two outpatient claims on separate service dates who were continuously enrolled between January 1, 2000 to December 31, 2008 were identified. ASD cases were matched to no ASD controls by age, sex, and race using propensity score matching (1:3). Baseline and outcome differences across cases and controls were examined using bivariate analyses for categorical (chi-square) and continuous independent variables (t-tests). All cause health care utilization (outpatient visits, inpatient hospitalizations, emergency room, and prescription drug use), and associated health care costs were assessed during the follow-up year. Multivariate multinomial logistic regressions for healthcare utilization and generalized linear models with gamma distribution for expenditures were conducted to compare healthcare utilization and expenditures among adults with and without ASD. Association of type of comorbidity with healthcare utilization (binary and multinomial logistic regressions) and 
PhD Dissertation Chapter 2

expenditures (generalized linear models with gamma distribution) were also analyzed among adults with ASD. Multivariate regression analyses were adjusted for state, identification year, patient socio-demographics, baseline prescription drug use, and county characteristics.

Results:

Comorbidities: Around $80 \%$ of adults with ASD $(n=1,772)$ had a psychiatric comorbidity as compared $41 \%$ of adults without ASD. Prevalence of a non-psychiatric comorbidity was significantly lower among adults with ASD (40\% ASD vs. $45 \%$ noASD). Almost $70 \%$ of adults with ASD had comorbid intellectual disability. Adults with ASD had higher rates of anxiety (12.2\% ASD vs. $5.6 \%$ noASD), attention deficit disorders/attention deficit hyperactivity disorders (ADD/ADHD) (8.2\% ASD vs. $0.7 \%$ noASD), schizophrenia (16.6\% ASD vs. $11.1 \%$ noASD), and gastrointestinal disorders (12.6\% ASD vs. $11 \%$ noASD) as compared to adults without ASD.

Rates of other health conditions that were significantly higher among adults with ASD as compared to adults without ASD were: epilepsy (22\% ASD Vs. 5\% noASD), thyroid disease (5\% ASD vs. $1 \%_{\text {noASD }}$ ), lipid metabolism disorders (5\% ASD Vs. 3\% noASD), other endocrine/nutrition disorders (11\% ASD vs. 4\% noASD) hematological disorders (7\% ASD Vs. 5\% noASD), genito-urinary disorders (10\% ASD vs. $8 \%$ noASD), infections (22\% ASD vs. 15\% noASD), skin disorders (21\% ASD vs. 9\% noASD), and paralysis (6\% ASD vs. 3\% noASD). Adults with ASD also had higher prevalence rate of other disabilities such as blindness (5\% ASD Vs. 4\% noASD) and hearing impairments (19\% ASD Vs. $4 \%$ noASD).

Healthcare utilization and expenditures: The mean number of OT visits ( $32_{\mathrm{ASD}}$ vs. 8 noASD) and Rx claims (51 ASD vs. 24noASD) per year were significantly greater for adults with ASD as compared to adults without ASD. The mean average length of stay was also higher for adults with ASD (10 ASD vs. 8 noASD days). Adults with ASD exhibited higher mean annual OT ( $\$ 4,375$ 
PhD Dissertation Chapter 2

ASD vs. $\$ 824$ noASD), ER ( $\$ 15,929$ ASD vs. $\$ 2,598$ noASD), Rx (\$6,067 ASD vs. $\$ 3,144$ noASD) and total expenditures ( $\$ 13,700$ ASD vs. $\left.\$ 8,560_{\text {noASD }}\right)$.

Adults with ASD were two times more likely to have $>=4$ OT visits per year as compared to adults without $\mathrm{ASD}(\mathrm{AOR}=2.73,95 \% \mathrm{CI}=2.31-3.22)$, five times more likely to have $>=18 \mathrm{Rx}$ claims than adults without $\mathrm{ASD}(\mathrm{AOR}=4.79,95 \% \mathrm{CI}=4.09-5.60)$. The likelihood of $>1 \mathrm{IP}$ visit $(\mathrm{AOR}=0.70,95 \% \mathrm{CI}=0.51-0.96)$ or $>1 \mathrm{ER}$ visit $(\mathrm{AOR}=0.76,95 \% \mathrm{CI}=0.61-0.93)$ in a year was $30 \%$ and $24 \%$ lower among adults with ASD as compared to adults without ASD. Among users, the expenditures for adults with ASD were significantly higher for OT visits, ER visits, and Rx claims as compared to adults without ASD after adjusting for selected characteristics. Adults with ASD had additional \$824 OT expenditures, \$8,932 ER expenditures, and \$1,499 Rx expenditures per year as compared to adults without ASD. The average adjusted total Medicaid expenditures (sum of OT, RX, and IP) were $\$ 1,159$ higher per year for adults with ASD as compared to adults without ASD, even after controlling for type of comorbidity. On an average, presence of a psychiatric and a non-psychiatric comorbidity among adults with ASD costed an additional $\$ 4,952$ and $\$ 5,084$ per year.

\section{Conclusion:}

Psychiatric comorbidities such as intellectual disabilities, anxiety, ADD/ADHD, and schizophrenia are very common among adults with ASD and non-psychiatric comorbidities such as gastrointestinal disorders are also highly common. Other than major comorbidities, other health conditions such as epilepsy, thyroid, lipid metabolism disorders, genito-urinary disorders, skin disorders, and infections are also highly prevalent in adults with ASD as compared to adults without ASD. Adults with ASD exhibit excess healthcare utilization in form of higher number of outpatient visits and prescription drug claims in a year and annual total Medicaid expenditures as 
PhD Dissertation Chapter 2

compared to adults without ASD. Medicaid coverage of healthcare services for adults with ASD is substantial and state programs bear high costs of such services. 
PhD Dissertation Chapter 2

\section{Introduction}

Comorbid conditions occurring among individuals with autism spectrum disorders (ASD) adversely affect their core autistic symptoms (Garcia-Villamisar \& Rojahn, 2013), activities of daily living, and health related quality of life (Kamp-Becker, Schroder, Remschmidt, \& Bachmann, 2010). Specifically for adults with ASD, the reported rates of comorbidities have varied substantially due to the variations in study designs, data sources, study period, type of ASD examined, and sample sizes. (Croen et al., 2015) used electronic health records of adults with ASD $(n=1,507)$ who were enrolled in Kaiser Permanente (KP) Northern California health insurance program from 2008 to 2012 and found very high rates of comorbid psychiatric as well as non-psychiatric conditions. Such comorbidities included depression (26\%), anxiety (30\%), gastrointestinal disorders (35\%), epilepsy (12\%) and diabetes (8\%). In addition, a few other studies have outlined the prevalence rates of comorbidities among adults with ASD using limited sample sizes and restricted hospital and/or community settings with a high chance of referral bias (Buck et al., 2014a; Lugnegard et al., 2011a; Lunsky et al., 2009). (Buck et al., 2014a) investigated comorbid psychiatric disorders among 129 adults with ASD ascertained during childhood in a 1980's statewide Utah autism prevalence study, and found that comorbid anxiety disorder had the highest current $(39.5 \%)$ and lifetime prevalence $(52.7 \%)$ in this group of adults. (Lugnegard et al., 2011a) examined the prevalence of psychiatric comorbidity in young adults diagnosed with Asperger's syndrome (mean age $=27$ years; 26 men and 28 women). In this study, major depression was shown to be as high as $70 \%$, followed by anxiety disorder (56\%), attention deficit hyperactivity disorder (ADHD; 30\%), substance dependence disorder (11\%), bipolar disorder (9\%), and obsessive compulsive disorder (OCD; 7\%). 
It is also well documented in the literature that children with ASD have higher healthcare resource utilization such as greater number of outpatient (OT) visits, inpatient hospitalizations (IP), length of stays, emergency room (ER) use, and medication (Rx) use and higher healthcare costs as compared to children without ASD (Croen et al., 2006; Lokhandwala, Khanna, \& WestStrum, 2012; Mandell, 2008a). For example, (Croen et al., 2006) examined KP medical care program 2003-2005 and found that children with ASD aged 2-18 year olds had a higher annual mean number of total clinic (5.6 vs 2.8$)$, pediatric (2.3 vs 1.6$)$, and psychiatric (2.2 vs 0.3 ) outpatient visits as compared to children without ASD. In addition, a higher percent of children with ASD also experienced inpatient hospitalizations (3\% vs 1\%) and were nine times more likely to use psychotherapeutic medications. Also, the mean annual member costs for hospitalizations (\$550 vs \$208), clinic visits (\$1373 vs \$540), and prescription medications (\$724 vs \$96) were significantly higher for children with ASD as compared to children without ASD. Since adults with ASD have similar autistic symptoms (Baker, 2013; Billstedt et al., 2007) and exhibit high healthcare needs (Cidav et al., 2013; Howlin, Goode, Hutton, \& Rutter, 2004; Lunsky et al., 2009; Mukaetova-Ladinska, Perry, Baron, Povey, \& Autism Ageing Writing Group, 2012b), it is important to establish their healthcare utilization and expenditures using real world settings.

Studies have also shown that comorbidities play a significant role in elevating the risk of using additional healthcare resources and increased healthcare expenditures among individuals with ASD (Ahmedani \& Hock, 2012; Buescher et al., 2014; Croen et al., 2006; Mandell et al., 2006; Mandell, 2008b; Peacock et al., 2012; Wang, Mandell, Lawer, Cidav, \& Leslie, 2012). Such studies have established that comorbidities along with ASD are specifically associated with higher medication use (Buck et al., 2014a; Mandell et al., 2008a; Mandell, 2008a), greater 
hospitalization rates, higher expenditures (Peacock et al., 2012) and extremely high lifetime support costs per individual with ASD (ASD alone $=\$ 1.4$ million and ASD+ Intellectual disability $=\$ 2.4$ million in US) (Buescher et al., 2014). Thus, it is critical to examine not only the burden of prevalence of comorbidities among adults with ASD, but also their ramifications on healthcare utilization and expenditures of adults with ASD. Given the recent studies which have established that individuals with ASD not only have psychiatric comorbid conditions but also non-psychiatric conditions (Buie et al., 2010; Croen et al., 2015; Esbensen et al., 2009; McElhanon et al., 2014), we include examining the rates of non-psychiatric disorders among adults with ASD as one of our study aims.

Even though the current literature indicates a high occurrence of specific comorbidities (mostly focusing on psychiatric) among adults with ASD (Buck et al., 2014a; Lugnegard et al., 2011a; Lunsky et al., 2009; Unenge Hallerback et al., 2012), there is a gap in the literature regarding how different these prevalence rates are from adults without ASD. The study by (Croen et al., 2015) is the first study that used a large sample of adults with ASD who were enrolled in KP Northern California to estimate and compare the prevalence rates of comorbidities among adults with and without ASD. Despite being a significant addition to the literature with a comprehensive understanding of prevalence rates of comorbidities among adults with ASD, this study was restricted to KP members in Northern California. In addition, the study also did not report the impact of each type of comorbidity among adults with ASD on their healthcare utilization and expenditures, which is of prime importance to policymakers as ASD related costs are predicted to increase substantially in the next few years (Buescher et al., 2014).

The current study will: 1) Examine the rates of specific types of psychiatric and nonpsychiatric comorbidities among adults with and without ASD; 2) Compare the healthcare 
utilization (OT, IP, ER, and Rx) and associated healthcare expenditures among adults with and without ASD; and 3) Determine the association of psychiatric and non-psychiatric comorbidity among adults with ASD with their healthcare utilization and expenditures. A psychiatric comorbidity is defined as any co-occurring diagnoses of psychiatric disorders such as adjustment disorders, alcohol/substance use disorders (AUD/SUD), anxiety disorders, attention deficit disorders \& conduct behavior disorders (ADD), developmental disorders, mood disorders including depressive disorders and bipolar disorder, personality disorders, and schizophrenia \& other psychotic disorders. A non-psychiatric comorbidity is defined as any co-occurring diagnoses of physical disorders such as cancer, cardiovascular conditions, diabetes, muscoskeletal disorders, gastrointestinal conditions, and respiratory conditions. We expect that adults with ASD will have a higher prevalence of both psychiatric and non-psychiatric comorbidities as compared to adults without ASD. Since autistic features persist into adulthood and continue to manifest symptoms of diagnostic autism (Billstedt et al., 2007; Seltzer et al., 2003), we also anticipate that adults with ASD will have greater use of healthcare services and have greater healthcare expenditures as compared to adults without ASD similar to findings of other studies among children with ASD (Ahmedani \& Hock, 2012; Buescher et al., 2014; Croen et al., 2006; Mandell et al., 2006; Mandell, 2008b; Peacock et al., 2012; Wang et al., 2012).

For the current study we used data from three state Medicaid (IL, NY, and TX) fee for services enrollees during the study period of 2000-2008. Medicaid is an ideal data source for several reasons. It is the single largest payer for individuals with ASD (Ruble et al., 2005) and utilization of Medicaid provided healthcare services among individuals with ASD is only expected to increase (Semansky et al., 2011a). Medicaid provides a wide variety of services to individuals with ASD (Arjun et al., 2011) including providing primary and secondary health 


\section{PhD Dissertation Chapter 2}

insurance coverage for healthcare services, home and community based waivers, screening and diagnostic services, behavioral support, in home care, skill training/acquisition, respite care, case management, supported employment, service coordination, self-directed services, and parent training and education (Mauch et al., 2011). Healthcare administrative claims, especially state Medicaid databases have been extensively used for ASD research among children and will help address similar questions for adults with ASD as well (Khanna et al., 2013; Mandell, 2008b; Maski et al., 2011; Peacock et al., 2012; Spencer et al., 2013). 
PhD Dissertation Chapter 2

\section{Method}

Study Population and Design

A retrospective matched cohort study among adults aged 22-64 years with and without ASD was conducted. Adults with any ASD (at least one inpatient or two outpatient claims on separate service dates with an ICD9-CM code: 299.xx in any position) and continuously enrolled in a fee for service program from 2000-2008 were matched (1:3) to adults without ASD by age, gender, and race using propensity score matching method with GREEDY (1 to 8) algorithm. Adults without ASD were included if they had at least two claims on separate dates of service (either inpatient or outpatient). Other exclusion criteria were: a) dual Medicaid/Medicare eligible; b) Managed care enrollees; and c) died during the observation period. The baseline characteristics (patient's socio-demographic information, comorbidities, and baseline drug use) were extracted from the identification year (first medical claim year with any ASD diagnosis) and the outcomes (healthcare utilization and expenditures) were measured in the follow up year (year after the identification year).

Data

Administrative health insurance claims data from three state Medicaid programs (IL, NY, and TX) were extracted from the 2000-2008 Centers for Medicare and Medicaid Services Medicaid analytic eXtract (MAX) data files. We selected IL, NY, and TX because of lower managed care penetration rates and greater fee-for-service enrollment during the study period (The Henry J Kaiser Family Foundation, 2013). The MAX files provide diagnosis codes, service use, demographic characteristics, and state of residence. Separate files received from CMS can be linked based on beneficiaries' unique identification number.

For the current study we used the personal summary file (provides information on Medicaid eligibility, enrollment type, and demographics), other therapy file (provides 
information on two possible diagnoses, utilization of outpatient services such as clinical services, physician services, procedures, home health, and lab services), inpatient file (provides information on nine possible diagnoses, hospitalizations, procedures, and length of stays), and the prescription drug file (national drug codes (NDC), prescription fill date, and days of supply). County level variables were obtained from the area resource file (ARF) (Quality Resource Systems, 2006) which provides information on type of health facilities, number and type of health professions, resource scarcity measures, health status, economic activities, health training programs, and socioeconomic and environmental characteristics. The Medicaid files were linked with ARF files with five digit county identification variable.

\section{Variables}

\section{Healthcare utilization and expenditures}

Our outcome measures were all cause healthcare utilization and expenditures in the follow up year. We counted the number of outpatient visits (OT), inpatient hospitalizations (IP), emergency use (ER), and any prescription drug claims $(\mathrm{Rx})$ on separate dates of service. OT services included all encounters and claims for services that were rendered in a doctor's office, hospital OT facility, ER, or other OT facility services. We defined the OT visits as any physician/other practitioner's/ clinic visits using MAX type of service codes. ER services were identified using revenue codes (450-452, 456, 459, 981), procedure codes (99281-99285), and place of service codes (23) provided in the MAX OT and IP file. Rx use included claims for prescriptions filled at retail and mail order pharmacies that were billed to the Medicaid's pharmacy benefits. In addition to visits, we included average length of stay for a hospitalization as one of the variables in the study. We also examined and compared the OT, IP, ER, Rx, and total expenditures (sum of OT, IP, and Rx expenditures) among adults with and without ASD. 
PhD Dissertation Chapter 2

Medical care services part of the annual consumer price index (CPI) was utilized to transform expenditures to 2008 constant dollars. The CPI was obtained from the Bureau of Labor Statistics (Bureau of Labor Statistics, US Department of Labor, 2014).

\section{Socio-demographic and need characteristics}

Age (22-40 and 41-64 years), sex (male and female), race (White, African-American, and Other), state (IL, NY, and TX), identification cohort (before and after 2004), and Medicaid eligibility by cash and medical needs (yes/no) were extracted from the MAX personal summary file. Baseline Rx use ( $>25$ unique Rx claims, $<=25$ unique Rx claims, and no Rx claims) was used as a proxy measure of healthcare needs.

\section{County Characteristics}

We used various contextual variables from the ARF file such as county metro status

(urban/rural), primary care shortage area (yes/no), mental health specialist shortage area (yes/no), psychiatrist density (high/low), median household income level (quartiles: q1, q2, q3, and q4), and high school education and above density which is a measure of county education status (quartiles: q1, q2, q3, and q4). These were used as a proxy measure of access to services.

\section{Comorbidities}

Two main types of comorbid conditions were identified using either one inpatient or two outpatient claims on separate service dates with a diagnosis in any position for a psychiatric or a non-psychiatric disorder. We used the single level clinical classification software (CCS) provided by Agency for Healthcare Research and Quality (https://www.hcup us.ahrq.gov/toolssoftware/CCS/AppendixASingleDX.txt) to categorize diagnosis codes to clinically meaningful categories. A psychiatric comorbidity included: adjustment disorders (CCS code: 650), alcohol/substance use disorders (AUD/SUD; CCS code: 660,661), anxiety disorders (CCS code: 651), attention deficit disorders \& conduct behavior disorders (ADD; CCS code: 
PhD Dissertation Chapter 2

652), developmental disorders (CCS code: 654), mood disorders including depressive disorders and bipolar disorder (CCS code: 6571,6572), personality disorders (CCS code: 658), and schizophrenia \& other psychotic disorders (CCS code: 659). A non-psychiatric comorbidity included: cancer (CCS code: 11- 37, 39-43, 46, 47) cardiovascular disease (CCS code: 96-108), diabetes (CCS code: 49, 50), muscoskeletal disorders (CCS code: 201-212), gastrointestinal disease (CCS code: 138-151, 153-155), and respiratory disease (CCS code: 125-128, 132-134). Other health conditions included epilepsy (CCS code: 83), headache including migraine (CCS code:84), thyroid disease (CCS code:48), other metabolic, nutritional, and endocrine disorders excluding diabetes (CCS code:51,58), nutrition deficiencies (CCS code:52), lipid metabolism disorders such as hypercholesterlomia (CCS code:53), fluid and electrolyte dysfunctions (CCS code:55), jaw and teeth disorders (CCS code:136), hematological disorders (CCS code: 59-64), genito-urinary disorders such as tract infections, bladder and urethra disorders, nephritis, renal failure, and others (CCS code: 156-163), paralysis (CCS code:82), infections such as tuberculosis, bacterial infections, hepatitis, HIV, and others (CCS code: 1-9,135), skin disorders such as infections, inflammatory conditions, and others (CCS code:197-200), blindness/other vision defects (CCS code:89), and hearing impairments (CCS code:92-94).

\section{Statistical Analysis}

Bivariate analyses such as chi-square tests of associations for categorical variables and ttests for continuous variables were conducted to assess the sub-group and mean differences between adults with and without ASD. Diagnostic tests for count data (OT, IP, ER, and Rx visits) were run to examine the feasibility of poisson, negative binomial, and zero inflated regressions. However, the dispersion factor for all regressions did not satisfy the distribution assumptions and count data were categorized into multinomial categories above and below the 
median of the sample distributions. For comparison between adults with and without ASD, a multinomial logistic regression was conducted to examine the likelihood of >=4 OT visits (vs. $<4$ OT visits and no OT visits), >1 IP visit (vs. =1 IP visit and no IP visit), >1 ER visit (vs. =1 ER visit and no ER visit), and >=18 Rx claims (vs. <18 Rx claims and no Rx claims) per year. We used generalized linear modeling with gamma distribution and log-link function to test for differences in Medicaid expenditures (OT, IP, ER, Rx, and total). To account for data with zero costs, a two-part model was used (part 1 with logistic regression to examine the association between all cause costs from ASD status [>0 vs 0] and part 2 with generalized linear modeling with log-link function for positive costs). For examining the association of type of comorbidity (psychiatric and non-psychiatric) with healthcare utilization and expenditures for adults with and without ASD, we used interaction terms (ASD*psychiatric comorbidity and ASD*nonpsychiatric comorbidity) in separate regression analyses. We tested the impact of comorbidity type in two ways: 1) Effect of comorbidity on differences in healthcare utilization and expenditures across adults with and without ASD (ASD with comorbidity vs. No ASD with comorbidity); and 2) Effect of comorbidity on healthcare utilization and expenditures of adults with ASD (ASD with comorbidity vs. ASD with no comorbidity).

Adjusted odds ratios (multinomial regressions) along with $95 \%$ confidence intervals, and parameter estimates (beta) with standard errors for generalized linear models are reported. All adjusted analyses were adjusted for socio-demographics, county characteristics, baseline Rx use, and comorbidities selectively. Variables which were highly collinear with expenditures such as association of baseline prescription drug use with Rx and total expenditures (sum of OT, IP, and Rx) were excluded from the model. Findings with cell sizes that were 11 or less are suppressed in accordance with CMS data user agreement. All analyses were conducted using SAS v9.4. 
PhD Dissertation Chapter 2

\section{Results}

Descriptive Analyses

Baseline Characteristics (Tables 1 and 2)

Our sample had 1,772 adults with ASD, most of them being male (71\%), aged 22-40

years, and under a cash assistance program (82\%). Around $70 \%$ of adults with ASD had more than 25 Rx claims per year as compared to $28 \%$ of adults without ASD. Psychiatric comorbidity rates were significantly higher among adults with ASD (81\%) than those without ASD (41\%). Almost $70 \%$ of adults with ASD in the sample had intellectual disability, followed by schizophrenia (17\%), mood disorders (14\%), and anxiety (12\%). Rates of non-psychiatric comorbidity were modestly lower among adults with ASD as compared to adults without ASD (40\% ASD vs. $46 \%$ noASD). Adults with ASD had significantly higher prevalence rate of gastrointestinal disorders (13\% ASD vs. 11\% noASD) and significantly lower prevalence rate for cardiovascular disorders (14\% ASD vs. 17\% noASD), muscoskeletal disorders (12\% ASD vs. 16\% noASD), and respiratory disorders (15\% ASD vs. $19 \%$ noASD) as compared to adults without ASD. Rates of other health conditions that were significantly higher among adults with ASD as compared to adults without ASD were: epilepsy (22\% ASD Vs. 5\% noASD), thyroid disease (5\% ASD vs. $1 \%_{\text {noASD }}$ ), lipid metabolism disorders (5\% ASD vs. 3\% noASD), other endocrine/nutrition disorders (11\% ASD Vs. 4\% noASD) hematological disorders (7\% ASD Vs. 5\% noASD), genito-urinary disorders (10\% ASD vs. $8 \%$ noASD), infections (22\% ASD vs. $15 \%$ noASD), skin disorders (21\% ASD vs. $9 \%$ noASD $)$, and paralysis (6\% ASD vs. $3 \%$ noASD). Adults with ASD also had higher prevalence rate of other disabilities such as blindness (5\% ASD vs. $4 \%$ noASD) and hearing impairments (19\% ASD vs. $4 \%$ noASD). 
PhD Dissertation Chapter 2

\section{Outcome Measures}

Most adults with ASD had an OT visit (95\%) in the follow up year (Table not shown here). Around $14 \%$ had an IP visit, 33.6\% had an ER visit, and 95\% had at least one Rx claim in the follow up year. IP (20\%) and ER visits (40\%) were much more common among adults without ASD as compared to adults with ASD. Almost $76 \%$ of adults with ASD used psychiatric OT services as compared to $41 \%$ of adults without ASD. Greater proportion of adults with ASD (35\%) also used residential OT services as compared to adults without ASD $(2 \%)$.

The mean number of OT visits ( $32_{\mathrm{ASD}}$ vs. $\left.8_{\text {noASD }}\right)$ and Rx claims ( $51_{\mathrm{ASD}}$ vs. $\left.24_{\text {noASD }}\right)$ per year were significantly greater for adults with ASD as compared to adults without ASD (Table 3a). The mean average length of stay was also higher for adults with ASD (10 ASD vs. 8 noASD days). Adults with ASD exhibited higher mean annual OT ( $\$ 4,375$ ASD vs. $\$ 824$ noASD), ER (\$15,929 ASD Vs. \$2,598 noASD), Rx (\$6,067 ASD vs. \$3,144 noASD) and total expenditures $(\$ 13,700$ ASD Vs. $\$ 8,560_{\text {noASD). }}$

Stratification of healthcare utilization and expenditures (Table not shown here) by each type of comorbidity showed higher OT and Rx visits per year as well as annual expenditures for most categories. Having a psychiatric comorbidity with ASD was associated with significantly higher mean OT visits (38 ASD vs. $11_{\text {noASD }}$ ) and Rx claims (55 $5_{\text {ASD }}$ vs. $32_{\text {noASD }}$ ) per year. Having a non-psychiatric comorbidity with ASD was also associated with higher mean OT visits (41 ASD vs. $10_{\text {noASD }}$ ) and Rx claims (62 $\mathrm{ASD}$ vs. $33_{\text {noASD }}$ ) per year. A similar pattern was observed for all expenditures, where annual OT, ER, and Rx expenditures were higher for the ASD group as compared to the no ASD group for all comorbidity groups. 
PhD Dissertation Chapter 2

Regression Analyses (Tables 4)

Adults with and without ASD

The adjusted odds ratios for all visits and claims were higher for adults with ASD as compared to adults without ASD, except for IP and ER visits. Adults with ASD were more likely to have >=4 OT visits per year as compared to adults without ASD after adjusting for sociodemographic, county, and need characteristics $(\mathrm{AOR}=2.73,95 \% \mathrm{CI}=2.31-3.22)$. Adults with ASD were five times more likely to have $>=18 \mathrm{Rx}$ claims than adults without $\mathrm{ASD}(\mathrm{AOR}=$ $4.79,95 \% \mathrm{CI}=4.09-5.60)$. Interestingly, the likelihood of $>1 \mathrm{IP}$ visit $(\mathrm{AOR}=0.70,95 \% \mathrm{CI}=$ $0.51-0.96)$ or $>1 \mathrm{ER}$ visit $(\mathrm{AOR}=0.76,95 \% \mathrm{CI}=0.61-0.93)$ in a year was $30 \%$ and $24 \%$ lower among adults with ASD as compared to adults without ASD. For healthcare expenditures, we conducted two part models to examine expenditure differences in adults with and without service use. ASD status, a key independent variable in the study was significantly associated with positive expenditures (non-zero) in the two part model. Adults with ASD were more likely to have positive expenditures for OT visits $(\mathrm{AOR}=3.67,95 \% \mathrm{CI}=2.89-4.66), \mathrm{Rx}$ claims $(\mathrm{AOR}=$ $2.24,95 \% \mathrm{CI}=1.75-2.87)$ and less likely to have positive expenditures for IP visits (AOR = $0.48,95 \% \mathrm{CI}=0.41-0.57)$ and $\mathrm{ER}$ visits $(\mathrm{AOR}=0.58,95 \% \mathrm{CI}=0.51-0.66)$. Among users, the expenditures for adults with ASD were significantly higher for OT visits, ER visits, and Rx claims as compared to adults without ASD after adjusting for selected characteristics. On an average (exp (intercept) - exp (beta)), adults with ASD had additional \$824 OT expenditures, $\$ 8,932$ ER expenditures, and \$1,499 Rx expenditures per year as compared to adults without ASD. The average adjusted total Medicaid expenditures (sum of OT, RX, and IP) were $\$ 1,159$ higher per year for adults with ASD as compared to adults without ASD, even after controlling for type of comorbidity 
Association of comorbidity with ASD status

Psychiatric comorbidity: Adults with ASD and a psychiatric comorbidity had significantly greater likelihood of having $>=4$ OT visits per year $(\mathrm{AOR}=3.05,95 \% \mathrm{CI}=2.49-3.73)$ and $>=$ $18 \mathrm{Rx}$ claims per year $(\mathrm{AOR}=3.87,95 \% \mathrm{CI}=3.23-4.65)$ as compared to adults without ASD but with a psychiatric comorbidity. Adults with ASD and a psychiatric comorbidity had lower likelihood of $>1 \mathrm{IP}$ visit $(\mathrm{AOR}=0.61,95 \% \mathrm{CI}=0.44-0.84)$ or $>1 \mathrm{ER}$ visit $(\mathrm{AOR}=0.71,95 \% \mathrm{CI}$ $=0.56-0.88)$ as compared to adults without ASD but with a psychiatric comorbidity. Among expenditures, adults with ASD and a psychiatric comorbidity had higher $\mathrm{OT}$ (beta $=1.14, \mathrm{SE}=$ 0.05, $\mathrm{p}<0.001), \mathrm{ER}(\mathrm{beta}=1.42, \mathrm{SE}=0.08, \mathrm{p}<0.001), \mathrm{Rx}($ beta $=0.32, \mathrm{SE}=0.05, \mathrm{p}<0.001)$, and total expenditures (beta $=0.19, \mathrm{SE}=0.05, \mathrm{p}<0.001$ ). Presence of psychiatric comorbidity among adults with ASD significantly increased the OT expenditures by $\$ 2,130$, ER expenditures by $\$ 10,532$, and total expenditures by $\$ 4,952$.

Non- Psychiatric comorbidity: Adults with a non-psychiatric comorbidity had significantly greater likelihood of having $>=4$ OT visits per year $(\mathrm{AOR}=2.48,95 \% \mathrm{CI}=1.91-3.22)$ and $>=$ $18 \mathrm{Rx}$ claims per year $(\mathrm{AOR}=5.43,95 \% \mathrm{CI}=4.19-7.04)$ as compared to adults without ASD but with a non-psychiatric comorbidity. Adults with ASD and a non-psychiatric comorbidity had no differences in likelihood of $>1$ IP visit or >1 ER visit as compared to adults without ASD but with a non-psychiatric comorbidity. Adults with ASD and a non-psychiatric comorbidity had higher OT $($ beta $=1.05, \mathrm{SE}=0.06, \mathrm{p}<0.001), \mathrm{ER}($ beta $=1.58, \mathrm{SE}=0.09, \mathrm{p}<0.001), \mathrm{Rx}(\mathrm{beta}=$ $0.54, \mathrm{SE}=0.06, \mathrm{p}<0.001)$, and total expenditures (beta $=0.40, \mathrm{SE}=0.06, \mathrm{p}<0.001)$ as compared to adults without ASD but with a non-psychiatric comorbidity. Presence of a non-psychiatric comorbidity among adults with ASD significantly increased the OT expenditures by $\$ 443$, IP expenditures by $\$ 1,990$, Rx expenditures by $\$ 1,160$, and total expenditures by $\$ 5,084$. 
PhD Dissertation Chapter 2 
PhD Dissertation Chapter 2

\section{Discussion}

Our study examined the rates of psychiatric and non-psychiatric comorbidities among adults with and without ASD in a 1:3 matched cohort. We also assessed differences in healthcare utilization and expenditures for adults with and without ASD, and examined the association of type of comorbidities with their healthcare utilization and expenditures. With an increasing number of adults with ASD (Brugha et al., 2011) and a lack of published studies on their healthcare needs, patterns of healthcare services use, and costs, this study fills a critical gap in the literature.

Although the rates of specific comorbidities among adults with ASD in our study are significantly different from those found in the recent study by (Croen et al., 2015), the two studies do share a common finding: prevalence of most psychiatric and a few non-psychiatric comorbidities among adults with ASD that are significantly higher when compared to adults without ASD. In addition, prevalence rates of several other co-occurring health conditions were also significantly higher among adults with ASD as compared to adults without ASD. Our rates of specific comorbidities as compared to the study by Croen et al. (2015) were much lower. For example around $26 \%$ and $30 \%$ of adults with ASD in the Croen et al. (2015) study had depression and anxiety as compared to $15 \%$ and $12 \%$ rates respectively in our study. We also found that rates of non-psychiatric comorbidities such as gastrointestinal disorders (35\% Croen vs. $12.6 \%$ ) and diabetes (7.6\% Croen vs. 3.6\%) in our sample were also lower in adults with ASD in our study. There could be many reasons for these differences in prevalence rates between the two studies: 1) Croen et al. (2015) focused only on KP members from Northern California, a population whose demographics may be inherently different from our three state (IL, NY, and TX) Medicaid population; 2) The data in Croen et al. (2015) study was more recent (2008-2012), which may represent a period of greater awareness of autism as well as better access to services 
associated with both mental and physical health needs (Patient Protection and Affordable Care Act, 42 U.S.C. $§ 18001$ (2010)); and 3) We used an algorithm of one inpatient or two outpatient claims to identify a comorbidity which may have excluded patients with a single claim only. This criteria, however, helped to create a more robust definition of a comorbid condition in our study along with reducing chances of including coding errors.

\section{Psychiatric comorbidities (Table 2)}

Similar to children with ASD (Buck et al., 2014; Gorrindo et al., 2012; Maski et al., 2011; Matson \& Shoemaker, 2009), adults with ASD also have very high rates of psychiatric comorbidities. Around $81 \%$ of adults with ASD had a psychiatric comorbidity as compared to $41 \%$ of adults without ASD. This prevalence rate of a psychiatric comorbidity also supports the findings from other small sample studies among adults with ASD (Buck et al., 2014a; Lugnegard et al., 2011a; Lunsky et al., 2009; Tsakanikos, Costello, Holt, Sturmey, \& Bouras, 2007). In our study the most common comorbid condition among adults with ASD was intellectual disability (ID) with a $70 \%$ prevalence rate. This finding is very critical since studies have shown how ASD with comorbid ID can increase the lifetime costs of individuals with ASD substantially (Buescher et al., 2014; Knapp et al., 2009).

Other common psychiatric comorbidities that had higher prevalence rates among adults with ASD as compared to adults without ASD were anxiety (12\% ASD vs. 5.6\% noASD) ADD/ADHD ( $8 \%_{\text {ASD }}$ vs. $0.7 \%_{\text {noASD }}$ ), and schizophrenia (16\% ASD vs. $\left.11 \%_{\text {noASD }}\right)$. Interestingly, behavioral disorders such as alcohol/substance abuse (2\% ASD vs. 18\% noASD) were significantly less common among adults with ASD as compared to adults without ASD. Literature has shown that usually individuals with ASD are less prone to use of drugs/alcohol, however, higher functioning individuals may frequently engage in alcohol consumption to help alleviate the 
social difficulties they experience (Santosh \& Mijovic, 2006; Sizoo, van den Brink, Gorissen van Eenige, \& van der Gaag, 2009). Since substance use disorder (SUD) is associated with greater healthcare resources utilization and worse outcomes (Smith, Stocks, \& Santora, 2015), greater attention and monitoring may be needed to identify SUD among adults with ASD so that timely counseling can be provided.

Non-psychiatric comorbidities (Table 2)

Adults with ASD had a significantly lower prevalence rate of non-psychiatric disorders at $40 \%$ as compared to $46 \%$ among adults without ASD. Even though a meta analyses showed higher prevalence of gastrointestinal (GI) disorders among children with ASD as compared to children without ASD (Buie et al., 2010; McElhanon et al., 2014), our study showed a significantly higher yet similar prevalence of GI symptoms among adults with ASD as compared to adults without ASD. The rate of cardiovascular/cerebrovascular disorders was significantly lower in adults without ASD (14\%) than adults with ASD (17\%). Muscoskeletal disorder prevalence rate was lower among adults with ASD (12\%) as compared to adults without ASD (16\%). It was the same case for diabetes rates as well, where around $4 \%$ of adults with ASD had a comorbid diabetes diagnosis as compared to $5 \%$ of adults without ASD. These findings reflect that adults with ASD if not higher, have similar extent of non-psychiatric needs as adults without ASD which are not usually addressed in the literature.

\section{Other Health Conditions}

Even though the rates of most non-psychiatric conditions except gastrointestinal disorders were much lower in adults with ASD as compared to adults without ASD, the prevalence of other comorbid health conditions were higher in the former group. For example, adults with ASD had significantly higher prevalence rates of epilepsy (22\%) as compared to adults without ASD (5\%), confirming the evidence on high epilepsy prevalence among individuals with ASD from 
previous studies (Howlin et al., 2004; Kohane et al., 2012; Maski et al., 2011). Other health conditions which were more common among adults with ASD were thyroid disease (5\%), other nutrition/endocrine/metabolic disorders excluding diabetes (11\%), lipid disorders (5\%), hematological disorders (6.5\%), infections (22\%), genito-urinary disorders (10\%), and skin disorders $(22 \%)$. Several of these disorders such as skin disorders have been found to be very common among children with ASD (Bakare, Munir, \& Kinney, 2011; Schieve et al., 2012). In addition, adults with ASD also showed higher prevalence of other impairments such as blindness/vision defects (5.4\%) and hearing impairments (19\%) showing high disability needs in this population.

There are a couple of notable points after examining prevalence rates of psychiatric, nonpsychiatric comorbidities, and other health conditions among adults with ASD. Psychiatric disorders such as schizophrenia and ASD have been linked previously in adult populations (Hofvander et al., 2009a; Mouridsen, Bronnum-Hansen, Rich, \& Isager, 2008). High rates of other psychiatric disorders such as anxiety and ADD should be highlighted in adults with ASD, since these disorders not only put the individuals at a greater risk of other disorders such as depression, but also are associated with suicide attempts (Kato et al., 2013). In addition many of these psychiatric conditions such as ADD/ADHD, mood disorders, and schizophrenia have been shown to share similar genetic makeup as compared to ASD in a recent study (Cross-Disorder Group of the Psychiatric Genomics Consortium, 2013). Pharmacotherapy for individuals with ASD, which usually includes antipsychotics, antidepressants, stimulants, and anticonvulsants have been shown to be associated with increased risk of dyslipidemia, metabolic syndrome, obesity, diabetes, and cardiovascular issues (Ji \& Findling, 2015; Rojo et al., 2015). Our study showed a high prevalence of lipid disorders which could be attributed to high medication use in 
the ASD population. Considering that a substantial proportion of adults with ASD diagnosed as children continue with these medications for a very long time (Esbensen et al., 2009), their risks of developing such conditions later in life are high (Croen et al., 2015). According to a study by (Nicolaidis et al., 2013), adults with ASD $(n=209)$ are more likely to report lower general and chronic condition self-efficacy, poor satisfaction rates with patient-provider communication, lower receipt of preventive services, and higher unmet needs as compared to adults without ASD ( $n=228)$. This study highlights that there are certain needs of adults with ASD that have not been met either due to difficulties in communication or lower access to ASD care. In addition, it is quite possible that social impairments may not allow adults with ASD to correctly report symptoms that cause discomfort, leading to possibly a missed or delayed diagnosis and foregone care.

\section{Health care utilization and expenditures (Table 3 and 4)}

In addition to assessing the rates of specific comorbidity in our sample, our study also examined the differences in use of healthcare services and associated expenditures across adults with and without ASD. We found that adults with ASD have significantly greater use of OT visits and Rx claims as compared to adults without ASD, regardless of the type of comorbidity associated with either group. The mean number of OT visits were 4 times higher and Rx claims were more than 2 times higher for adults with ASD as compared to adults without ASD. Surprisingly, IP visits were much lower in ASD group and there were no significant differences in the mean number of ER visits among adults with and without ASD despite previous studies among individuals with ASD of all ages as compared to individuals without ASD showing a higher inpatient burden (Croen et al., 2006; Lokhandwala et al., 2012). Previous studies have shown that individuals with ASD who participate in home and community based (HCBS) waivers have significantly lower likelihood of using IP or long term care services (Cidav, 
Marcus, \& Mandell, 2014; Velott et al., 2015). It is quite possible that many individuals with ASD in our sample are also participating in HCBS waivers and making their hospitalization rates are lower as compared to adults without ASD.

Adults with ASD also had significantly higher mean total expenditures (sum of OT, IP, and Rx expenditures) as compared to total expenditures of adults without ASD. The mean OT, ER, Rx, and total expenditures associated with adults with ASD were 5 times, 7 times, 2 times, and 2 times higher, respectively than adults without ASD (Table 3). In contrast to lower mean number of ER visits among adults with ASD, the corresponding ER expenditures were significantly higher than for adults without ASD. After adjusting for selected characteristics, adults with ASD had 0.4 times $(\$ 1,159$ difference; Table 4) the average annual total expenditures of adults without ASD. There are a plethora of studies that have shown that children with ASD have high healthcare utilization and expenditures, especially as compared to children without ASD (Croen et al., 2006; Kogan et al., 2008; Leslie \& Martin, 2007; Liptak et al., 2006; Lokhandwala et al., 2012; Mandell et al., 2006; Shimabukuro, Grosse, \& Rice, 2008; Wang \& Leslie, 2010a). Our study is the first among a large sample of adults with ASD identifying the excess healthcare utilization and expenditures as compared to adults without ASD. The reasons why the healthcare utilization and expenditures are higher among the ASD group may be attributable to the extensive comorbidities among adults with ASD and the lack of knowledge of ASD among healthcare providers which leads to delayed care and frequent revisits to the outpatient settings, ER, and hospitals (Heidgerken et al., 2005; Imran et al., 2011).

Our study also contributed to the literature by providing an estimate of the association of the type of comorbidity with healthcare utilization and expenditures of adults with and without ASD (Table 4). There was no significant impact of psychiatric comorbidity on likelihood of 
healthcare utilization per year, where adults with ASD showed significantly higher OT and Rx utilization and lower IP and ER utilization as compared to adults without ASD. However, nonpsychiatric comorbidity did have an effect modification on likelihood of higher IP and ER visits, where adults with and without ASD were no longer significantly different. This finding could mean that non-psychiatric comorbidity contributes significantly to determining the extent of hospitalizations and ER visits among adults with and without ASD. In terms of differential economic burden due to comorbidities, adults with ASD had consistently higher OT, ER, Rx, and total expenditures and lower IP expenditures as compared to adults without ASD, regardless of type of comorbidity.

Presence of comorbidity within adults with ASD did have a significant impact on both healthcare utilization as well as expenditures. Presence of psychiatric comorbidity among adults with ASD significantly increased the OT expenditures by $\$ 2,130$, ER expenditures by $\$ 10,532$, and total expenditures by $\$ 4,952$. Presence of a non-psychiatric comorbidity among adults with ASD significantly increased the OT expenditures by $\$ 443$, IP expenditures by $\$ 1,990, \mathrm{Rx}$ expenditures by $\$ 1,160$, and total expenditures by $\$ 5,084$. (Peacock et al., 2012) established that Medicaid enrolled children with ASD have substantially higher healthcare expenditures based on the type of comorbid condition. Having ADHD, epilepsy, and intellectual disability was associated with 1.3 times, 1.6 times, and 2.7 times higher annual total expenditures for adults with ASD as compared to adults with ASD but no comorbidity.

\section{Implications}

Our study showed that adults with ASD not only suffer from a great deal of comorbid pathology but also exhibit high healthcare needs and expenditures. Addressing the core deficit areas in adult ASD care is the next step where adults will have a better transition from childhood, 
are able to communicate their issues to a healthcare provider, and receive well-coordinated and quality care for psychiatric, non-psychiatric conditions, and other health conditions. From a policy perspective, our study showed that adults with ASD represent a high needs group within the Medicaid population. Medicaid coverage provides substantial number of services for adults with ASD which also transforms into extremely high costs. With the recent efforts to reduce long term healthcare costs and still maintain quality care, understanding the pattern of healthcare utilization and factors influencing the high services use among adults with ASD may draw attention to the need for better coordinated care and/or processes to improve communication and treatment experiences.

As noted in many previous studies (Heidgerken et al., 2005; Imran et al., 2011; Liptak et al., 2006; Miller, 2015; Piven, Rabins, \& Autism-in-Older Adults Working Group, 2011), ASD knowledge and training among physicians and other specialty care providers have been reported to be low, and there is a lack in self-reported confidence among providers in being able to correctly identify ASD, understand their symptoms and pharmacological needs, and provide quality care that improves disease prognosis and reduce morbidity. In a scenario where there is no gold standard cure and most medications have medium to low level effectiveness (Warren et al., April 2011), greater focus may be needed to provide easy access to early intervention services which have been shown to significantly improve prognosis of this condition (Autism Speaks, 2013; Bailey, Hebbeler, Scarborough, Spiker, \& Mallik, 2004; Reichow, 2012). In addition, further studies may be needed to address the poor rating of patient provider communication and greater unmet healthcare needs that have been reported by adults with ASD (Magiati et al., 2014; Nicolaidis et al., 2013). 
PhD Dissertation Chapter 2

\section{Limitations}

To conduct this study, we used a three state Medicaid dataset (IL, NY, and TX) for the period 2000-2008. Even though these states provided variation in demographics and high number of adults with ASD, we did not control for any unobservable bias in the study. The goal of the study was to examine the rates of comorbidities and excess healthcare use and expenditures among adults with ASD as compared to adults without ASD and our current data was sufficient for this purpose. Our identification of ASD and comorbidities using a conservative algorithm has not been validated among adults and this study may underestimate the real world prevalence of adults with ASD as well as comorbidities in Medicaid data. This was also an issue because there are no existing published studies on adults with ASD in the Medicaid population which could be used as a reference point. Our study focused on all cause healthcare utilization and expenditures, so we did not know what type of diagnoses these outcomes were associated with or what were the reasons of such visits. We required adults with ASD to be continuously enrolled during the study period to allow capturing their true healthcare utilization and expenditures, which could have excluded many individuals with irregular Medicaid enrollment. We did not match the ASD cases and no ASD controls on their enrollment period, which could have created certain baseline differences in the two groups.

\section{Strengths}

The current study is the first study to examine a large number of adults with ASD and compare their extent of comorbidities, healthcare utilization, and expenditures to adults without ASD within a Medicaid population. Medicaid serves as the largest single payer for individuals with ASD (Ruble et al., 2005) and highlighting the healthcare use patterns and expenditures associated with this population will help promote better education and development of guidelines for healthcare providers on ASD treatment and care. We also examined the impact of the type of 
PhD Dissertation Chapter 2

comorbidity on healthcare utilization and expenditures for adults with ASD to provide an estimate of how a comorbidity can impact the healthcare use of adults with ASD. We addressed significant observed differences between adults with and without ASD by matching them by age, gender, and race.

\section{Conclusions}

Psychiatric comorbidities such as intellectual disabilities, anxiety, ADD/ADHD, and schizophrenia are very common among adults with ASD and non-psychiatric comorbidities such as gastrointestinal disorders are also highly common. Other than major comorbidities, other health conditions such as epilepsy, thyroid, lipid metabolism disorders, genito-urinary disorders, skin disorders, and infections are also highly prevalent in adults with ASD as compared to adults without ASD. Adults with ASD exhibit excess healthcare utilization in form of higher number of outpatient visits and prescription drug claims in a year and annual total Medicaid expenditures as compared to adults without ASD. Even though comorbidities play a significant role in increasing service utilization and expenditures for adults with ASD, they contribute minimally towards the differences in healthcare utilization and expenditures between adults with and without ASD. Medicaid coverage of healthcare services for adults with ASD is substantial and state programs bear high costs of such services. Care should be directed towards managing both psychiatric and non-psychiatric needs of adults with ASD in the primary as well as specialty care settings. Future policies should strive for better ASD training and education of healthcare providers as well as guidelines for treatment that assure quality and well-coordinated care for adults with ASD. 


\section{PhD Dissertation Chapter 2}

\section{References}

Ahmedani, B. K., \& Hock, R. M. (2012). Health care access and treatment for children with co-morbid autism and psychiatric conditions. Social Psychiatry and Psychiatric Epidemiology, 47(11), 1807-1814. doi:10.1007/s00127-012-0482-0; 10.1007/s00127-012-0482-0

Arjun, L., Hope, C., Guyer, J., \& Alker, J. (2011). Medicaid and its role for children and youth with special health care needs (CYSHCN): A family perspective. (). Georgetown University, Health Policy Institute: Center for Children and Families/Family Voices.

Autism Speaks. (2013). High-quality early intervention for autism more than pays for itself. Retrieved from http://www.autismspeaks.org/science/science-news/high-quality-early-intervention-autism-more-pays-itself

Bailey, D. B.,Jr, Hebbeler, K., Scarborough, A., Spiker, D., \& Mallik, S. (2004). First experiences with early intervention: A national perspective. Pediatrics, 113(4), 887-896.

Bakare, M. O., Munir, K. M., \& Kinney, D. K. (2011). Association of hypomelanotic skin disorders with autism: Links to possible etiologic role of vitamin-D levels in autism? Hypothesis (University of Toronto.Dept.of Medical Biophysics), 9(1), e2. doi:e2 [pii]

Baker, J. P. (2013). Autism at 70--redrawing the boundaries. The New England Journal of Medicine, 369(12), 10891091. doi:10.1056/NEJMp1306380; 10.1056/NEJMp1306380

Billstedt, E., Gillberg, I. C., \& Gillberg, C. (2007). Autism in adults: Symptom patterns and early childhood predictors. use of the DISCO in a community sample followed from childhood. Journal of Child Psychology and Psychiatry, and Allied Disciplines, 48(11), 1102-1110. doi:JCPP1774 [pii]

Brugha, T. S., McManus, S., Bankart, J., Scott, F., Purdon, S., Smith, J., . . Meltzer, H. (2011). Epidemiology of autism spectrum disorders in adults in the community in england. Archives of General Psychiatry, 68(5), 459465. doi:10.1001/archgenpsychiatry.2011.38; 10.1001/archgenpsychiatry.2011.38

Buck, T. R., Viskochil, J., Farley, M., Coon, H., McMahon, W. M., Morgan, J., \& Bilder, D. A. (2014). Psychiatric comorbidity and medication use in adults with autism spectrum disorder. Journal of Autism and Developmental Disorders, doi:10.1007/s10803-014-2170-2 [doi]

Buescher, A. V., Cidav, Z., Knapp, M., \& Mandell, D. S. (2014). Costs of autism spectrum disorders in the united kingdom and the united states. JAMA Pediatrics, 168(8), 721-728. doi:10.1001/jamapediatrics.2014.210 [doi]

Buie, T., Campbell, D. B., Fuchs, G. J.,3rd, Furuta, G. T., Levy, J., Vandewater, J., . . Winter, H. (2010). Evaluation, diagnosis, and treatment of gastrointestinal disorders in individuals with ASDs: A consensus report. Pediatrics, 125 Suppl 1, S1-18. doi:10.1542/peds.2009-1878C [doi]

Bureau of Labor Statistics, US Department of Labor. (2014). Archived consumer price index detailed report information. Retrieved from http://www.bls.gov/cpi/cpi_dr.htm

Cidav, Z., Lawer, L., Marcus, S. C., \& Mandell, D. S. (2013). Age-related variation in health service use and associated expenditures among children with autism. Journal of Autism and Developmental Disorders, 43(4), 924-931. doi:10.1007/s10803-012-1637-2 [doi]

Cidav, Z., Marcus, S. C., \& Mandell, D. S. (2014). Home- and community-based waivers for children with autism: Effects on service use and costs. Intellectual and Developmental Disabilities, 52(4), 239-248. doi:10.1352/1934-9556-52.4.239 [doi] 


\section{PhD Dissertation Chapter 2}

Croen, L. A., Najjar, D. V., Ray, G. T., Lotspeich, L., \& Bernal, P. (2006). A comparison of health care utilization and costs of children with and without autism spectrum disorders in a large group-model health plan. Pediatrics, 118(4), e1203-11. doi:118/4/e1203 [pii]

Croen, L. A., Zerbo, O., Qian, Y., Massolo, M. L., Rich, S., Sidney, S., \& Kripke, C. (2015). The health status of adults on the autism spectrum. Autism : The International Journal of Research and Practice, doi:1362361315577517 [pii]

Cross-Disorder Group of the Psychiatric Genomics Consortium. (2013). Identification of risk loci with shared effects on five major psychiatric disorders: A genome-wide analysis. Lancet, 381(9875), 1371-1379. doi:10.1016/S0140-6736(12)62129-1 [doi]

Esbensen, A. J., Greenberg, J. S., Seltzer, M. M., \& Aman, M. G. (2009). A longitudinal investigation of psychotropic and non-psychotropic medication use among adolescents and adults with autism spectrum disorders. Journal of Autism and Developmental Disorders, 39(9), 1339-1349. doi:10.1007/s10803-009-07503 [doi]

Garcia-Villamisar, D., \& Rojahn, J. (2013). Comorbid psychopathology and stress mediate the relationship between autistic traits and repetitive behaviours in adults with autism. Journal of Intellectual Disability Research : $J I D R$, doi:10.1111/jir.12083 [doi]

Heidgerken, A. D., Geffken, G., Modi, A., \& Frakey, L. (2005). A survey of autism knowledge in a health care setting. Journal of Autism and Developmental Disorders, 35(3), 323-330.

Hofvander, B., Delorme, R., Chaste, P., Nyden, A., Wentz, E., Stahlberg, O., . . Leboyer, M. (2009). Psychiatric and psychosocial problems in adults with normal-intelligence autism spectrum disorders. BMC Psychiatry, 9, 35-244X-9-35. doi:10.1186/1471-244X-9-35 [doi]

Howlin, P., Goode, S., Hutton, J., \& Rutter, M. (2004). Adult outcome for children with autism. Journal of Child Psychology and Psychiatry, and Allied Disciplines, 45(2), 212-229.

Imran, N., Chaudry, M. R., Azeem, M. W., Bhatti, M. R., Choudhary, Z. I., \& Cheema, M. A. (2011). A survey of autism knowledge and attitudes among the healthcare professionals in lahore, pakistan. BMC Pediatrics, 11, 107-2431-11-107. doi:10.1186/1471-2431-11-107 [doi]

Ji, N., \& Findling, R. L. (2015). An update on pharmacotherapy for autism spectrum disorder in children and adolescents. Current Opinion in Psychiatry, 28(2), 91-101. doi:10.1097/YCO.0000000000000132 [doi]

Kamp-Becker, I., Schroder, J., Remschmidt, H., \& Bachmann, C. J. (2010). Health-related quality of life in adolescents and young adults with high functioning autism-spectrum disorder. Psycho-Social Medicine, 7 , 10.3205/psm000065. doi:10.3205/psm000065 [doi]

Kato, K., Mikami, K., Akama, F., Yamada, K., Maehara, M., Kimoto, K., . . Matsumoto, H. (2013). Clinical features of suicide attempts in adults with autism spectrum disorders. General Hospital Psychiatry, 35(1), 5053. doi:10.1016/j.genhosppsych.2012.09.006 [doi]

Khanna, R., Jariwala, K., \& West-Strum, D. (2013). Use and cost of psychotropic drugs among recipients with autism in a state medicaid fee-for-service programme. Journal of Intellectual Disability Research : JIDR, 57(2), 161-171. doi:10.1111/j.1365-2788.2012.01563.x; 10.1111/j.1365-2788.2012.01563.x

Knapp, M., Romeo, R., \& Beecham, J. (2009). Economic cost of autism in the UK. Autism : The International Journal of Research and Practice, 13(3), 317-336. doi:10.1177/1362361309104246 [doi] 


\section{PhD Dissertation Chapter 2}

Kogan, M. D., Strickland, B. B., Blumberg, S. J., Singh, G. K., Perrin, J. M., \& van Dyck, P. C. (2008). A national profile of the health care experiences and family impact of autism spectrum disorder among children in the united states, 2005-2006. Pediatrics, 122(6), e1149-58. doi:10.1542/peds.2008-1057; 10.1542/peds.2008-1057

Kohane, I. S., McMurry, A., Weber, G., MacFadden, D., Rappaport, L., Kunkel, L., . . Churchill, S. (2012). The co-morbidity burden of children and young adults with autism spectrum disorders. PloS One, 7(4), e33224. doi:10.1371/journal.pone.0033224; 10.1371/journal.pone.0033224

Leslie, D. L., \& Martin, A. (2007). Health care expenditures associated with autism spectrum disorders. Archives of Pediatrics \& Adolescent Medicine, 161(4), 350-355. doi:10.1001/archpedi.161.4.350

Liptak, G. S., Orlando, M., Yingling, J. T., Theurer-Kaufman, K. L., Malay, D. P., Tompkins, L. A., \& Flynn, J. R. (2006). Satisfaction with primary health care received by families of children with developmental disabilities. Journal of Pediatric Health Care : Official Publication of National Association of Pediatric Nurse Associates \& Practitioners, 20(4), 245-252. doi:10.1016/j.pedhc.2005.12.008

Liptak, G. S., Stuart, T., \& Auinger, P. (2006). Health care utilization and expenditures for children with autism: Data from U.S. national samples. Journal of Autism and Developmental Disorders, 36(7), 871-879. doi:10.1007/s10803-006-0119-9

Lokhandwala, T., Khanna, R., \& West-Strum, D. (2012). Hospitalization burden among individuals with autism. Journal of Autism and Developmental Disorders, 42(1), 95-104. doi:10.1007/s10803-011-1217-x [doi]

Lugnegard, T., Hallerback, M. U., \& Gillberg, C. (2011). Psychiatric comorbidity in young adults with a clinical diagnosis of asperger syndrome. Research in Developmental Disabilities, 32(5), 1910-1917. doi:10.1016/j.ridd.2011.03.025 [doi]

Lunsky, Y., Gracey, C., \& Bradley, E. (2009). Adults with autism spectrum disorders using psychiatric hospitals in ontario: Clinical profile and service needs. Research in Autism Spectrum Disorders, 3(4), 1006-1013.

Magiati, I., Tay, X. W., \& Howlin, P. (2014). Cognitive, language, social and behavioural outcomes in adults with autism spectrum disorders: A systematic review of longitudinal follow-up studies in adulthood. Clinical Psychology Review, 34(1), 73-86. doi:10.1016/j.cpr.2013.11.002 [doi]

Mandell, D. S. (2008a). Psychiatric hospitalization among children with autism spectrum disorders. Journal of Autism and Developmental Disorders, 38(6), 1059-1065. doi:10.1007/s10803-007-0481-2 [doi]

Mandell, D. S. (2008b). Psychiatric hospitalization among children with autism spectrum disorders. Journal of Autism and Developmental Disorders, 38(6), 1059-1065. doi:10.1007/s10803-007-0481-2 [doi]

Mandell, D. S., Cao, J., Ittenbach, R., \& Pinto-Martin, J. (2006). Medicaid expenditures for children with autistic spectrum disorders: 1994 to 1999. Journal of Autism and Developmental Disorders, 36(4), 475-485. doi:10.1007/s10803-006-0088-z

Mandell, D. S., Morales, K. H., Marcus, S. C., Stahmer, A. C., Doshi, J., \& Polsky, D. E. (2008). Psychotropic medication use among medicaid-enrolled children with autism spectrum disorders. Pediatrics, 121(3), e441-8. doi:10.1542/peds.2007-0984; 10.1542/peds.2007-0984

Maski, K. P., Jeste, S. S., \& Spence, S. J. (2011). Common neurological co-morbidities in autism spectrum disorders. Current Opinion in Pediatrics, 23(6), 609-615. doi:10.1097/MOP.0b013e32834c9282; 10.1097/MOP.0b013e32834c9282 


\section{PhD Dissertation Chapter 2}

Mauch, D., Pfefferle, S., Booker, C., Pustell, M., \& Levin, J. (2011). Report on state services to individuals with autism spectrum disorders (ASD). ( No. S-10 CMS-33 No. 2). Centers for Medicare \& Medicaid Services (CMS) ASD Services Project: Centers for Medicare \& Medicaid Services (CMS).

McElhanon, B. O., McCracken, C., Karpen, S., \& Sharp, W. G. (2014). Gastrointestinal symptoms in autism spectrum disorder: A meta-analysis. Pediatrics, doi:peds.2013-3995 [pii]

Miller, S. M. (2015). Nurses caring for adults with autism in an emergency department: A survey of knowledge. Doctoral Projects, (7)

Mouridsen, S. E., Bronnum-Hansen, H., Rich, B., \& Isager, T. (2008). Mortality and causes of death in autism spectrum disorders: An update. Autism : The International Journal of Research and Practice, 12(4), 403-414. doi:10.1177/1362361308091653 [doi]

Mukaetova-Ladinska, E. B., Perry, E., Baron, M., Povey, C., \& Autism Ageing Writing Group. (2012). Ageing in people with autistic spectrum disorder. International Journal of Geriatric Psychiatry, 27(2), 109-118. doi:10.1002/gps.2711; 10.1002/gps.2711

Nicolaidis, C., Raymaker, D., McDonald, K., Dern, S., Boisclair, W. C., Ashkenazy, E., \& Baggs, A. (2013). Comparison of healthcare experiences in autistic and non-autistic adults: A cross-sectional online survey facilitated by an academic-community partnership. Journal of General Internal Medicine, 28(6), 761-769. doi:10.1007/s11606-012-2262-7 [doi]

Peacock, G., Amendah, D., Ouyang, L., \& Grosse, S. D. (2012). Autism spectrum disorders and health care expenditures: The effects of co-occurring conditions. Journal of Developmental and Behavioral Pediatrics : JDBP, 33(1), 2-8. doi:10.1097/DBP.0b013e31823969de; 10.1097/DBP.0b013e31823969de

Piven, J., Rabins, P., \& Autism-in-Older Adults Working Group. (2011). Autism spectrum disorders in older adults: Toward defining a research agenda. Journal of the American Geriatrics Society, 59(11), 2151-2155. doi:10.1111/j.1532-5415.2011.03632.x [doi]

Quality Resource Systems, I. (2006). Area resource file. (). Fairfax, VA: Health Resources and Services Administration, US Department of Health and Human Services, 2006.

Reichow, B. (2012). Overview of meta-analyses on early intensive behavioral intervention for young children with autism spectrum disorders. Journal of Autism and Developmental Disorders, 42(4), 512-520. doi:10.1007/s10803-011-1218-9; 10.1007/s10803-011-1218-9

Rojo, L. E., Gaspar, P. A., Silva, H., Risco, L., Arena, P., Cubillos-Robles, K., \& Jara, B. (2015). Metabolic syndrome and obesity among users of second generation antipsychotics: A global challenge for modern psychopharmacology. Pharmacological Research : The Official Journal of the Italian Pharmacological Society, doi:S1043-6618(15)00155-3 [pii]

Ruble, L. A., Heflinger, C. A., Renfrew, J. W., \& Saunders, R. C. (2005). Access and service use by children with autism spectrum disorders in medicaid managed care. Journal of Autism and Developmental Disorders, 35(1), 3-13.

Santosh, P. J., \& Mijovic, A. (2006). Does pervasive developmental disorder protect children and adolescents against drug and alcohol use? European Child \& Adolescent Psychiatry, 15(4), 183-188. doi:10.1007/s00787005-0517-0 [doi]

Schieve, L. A., Gonzalez, V., Boulet, S. L., Visser, S. N., Rice, C. E., Van Naarden Braun, K., \& Boyle, C. A. (2012). Concurrent medical conditions and health care use and needs among children with learning and 


\section{PhD Dissertation Chapter 2}

behavioral developmental disabilities, national health interview survey, 2006-2010. Research in Developmental Disabilities, 33(2), 467-476. doi:10.1016/j.ridd.2011.10.008 [doi]

Seltzer, M. M., Krauss, M. W., Shattuck, P. T., Orsmond, G., Swe, A., \& Lord, C. (2003). The symptoms of autism spectrum disorders in adolescence and adulthood. Journal of Autism and Developmental Disorders, 33(6), $565-581$.

Semansky, R. M., Xie, M., \& Mandell, D. S. (2011). Medicaid's increasing role in treating youths with autism spectrum disorders. Psychiatric Services (Washington, D.C.), 62(6), 588. doi:10.1176/appi.ps.62.6.588; 10.1176/appi.ps.62.6.588

Shimabukuro, T. T., Grosse, S. D., \& Rice, C. (2008). Medical expenditures for children with an autism spectrum disorder in a privately insured population. Journal of Autism and Developmental Disorders, 38(3), 546-552. doi:10.1007/s10803-007-0424-y

Sizoo, B., van den Brink, W., Gorissen van Eenige, M., \& van der Gaag, R. J. (2009). Personality characteristics of adults with autism spectrum disorders or attention deficit hyperactivity disorder with and without substance use disorders. The Journal of Nervous and Mental Disease, 197(6), 450-454. doi:10.1097/NMD.0b013e3181a61dd0 [doi]

Smith, M. W., Stocks, C., \& Santora, P. B. (2015). Hospital readmission rates and emergency department visits for mental health and substance abuse conditions. Community Mental Health Journal, 51(2), 190-197. doi:10.1007/s10597-014-9784-x [doi]

Spencer, D., Marshall, J., Post, B., Kulakodlu, M., Newschaffer, C., Dennen, T., . . Jain, A. (2013). Psychotropic medication use and polypharmacy in children with autism spectrum disorders. Pediatrics, 132(5), 833-840. doi:10.1542/peds.2012-3774; 10.1542/peds.2012-3774

The Henry J Kaiser Family Foundation. (2013). State HMO penetration rate, state health facts. Retrieved from http://kff.org/other/state-indicator/hmo-penetration-rate/

Tsakanikos, E., Costello, H., Holt, G., Sturmey, P., \& Bouras, N. (2007). Behaviour management problems as predictors of psychotropic medication and use of psychiatric services in adults with autism. Journal of Autism and Developmental Disorders, 37(6), 1080-1085. doi:10.1007/s10803-006-0248-1 [doi]

Unenge Hallerback, M., Lugnegard, T., \& Gillberg, C. (2012). Is autism spectrum disorder common in schizophrenia? Psychiatry Research, 198(1), 12-17. doi:10.1016/j.psychres.2012.01.016; 10.1016/j.psychres.2012.01.016

Velott, D. L., Agbese, E., Mandell, D., Stein, B. D., Dick, A. W., Yu, H., \& Leslie, D. L. (2015). Medicaid 1915(c) home- and community-based services waivers for children with autism spectrum disorder. Autism : The International Journal of Research and Practice, doi:1362361315590806 [pii]

Wang, L., \& Leslie, D. L. (2010). Health care expenditures for children with autism spectrum disorders in medicaid. Journal of the American Academy of Child and Adolescent Psychiatry, 49(11), 1165-1171. doi:10.1016/j.jaac.2010.08.003; 10.1016/j.jaac.2010.08.003

Wang, L., Mandell, D. S., Lawer, L., Cidav, Z., \& Leslie, D. L. (2012). Healthcare service use and costs for autism spectrum disorder: A comparison between medicaid and private insurance. Journal of Autism and Developmental Disorders, doi:10.1007/s10803-012-1649-y

Warren, Z., Veenstra-VanderWeele, J., Stone, W., Bruzek, J. L., Nahmias, A. S., Foss-Feig, J. H., . . McPheeters, M. L. (April 2011). Therapies for children with autism spectrum disorders.. (Comparative Effectiveness 


\section{PhD Dissertation Chapter 2}

Reviews no. 26 No. 11-EHC029-EF). Rockville (MD): Agency for Healthcare Research and Quality. doi:http://effectivehealthcare.ahrq.gov/ehc/products/106/651/Autism_Disorder_exec-summ.pdf 
PhD Dissertation Chapter 2

Tables

Table 1. Description of Sample by ASD diagnoses

Adults with and without ASD matched on age, race, and gender $(n=7,092)$

Medicaid Analytic Extract 2000-2008 (IL,NY, and TX)

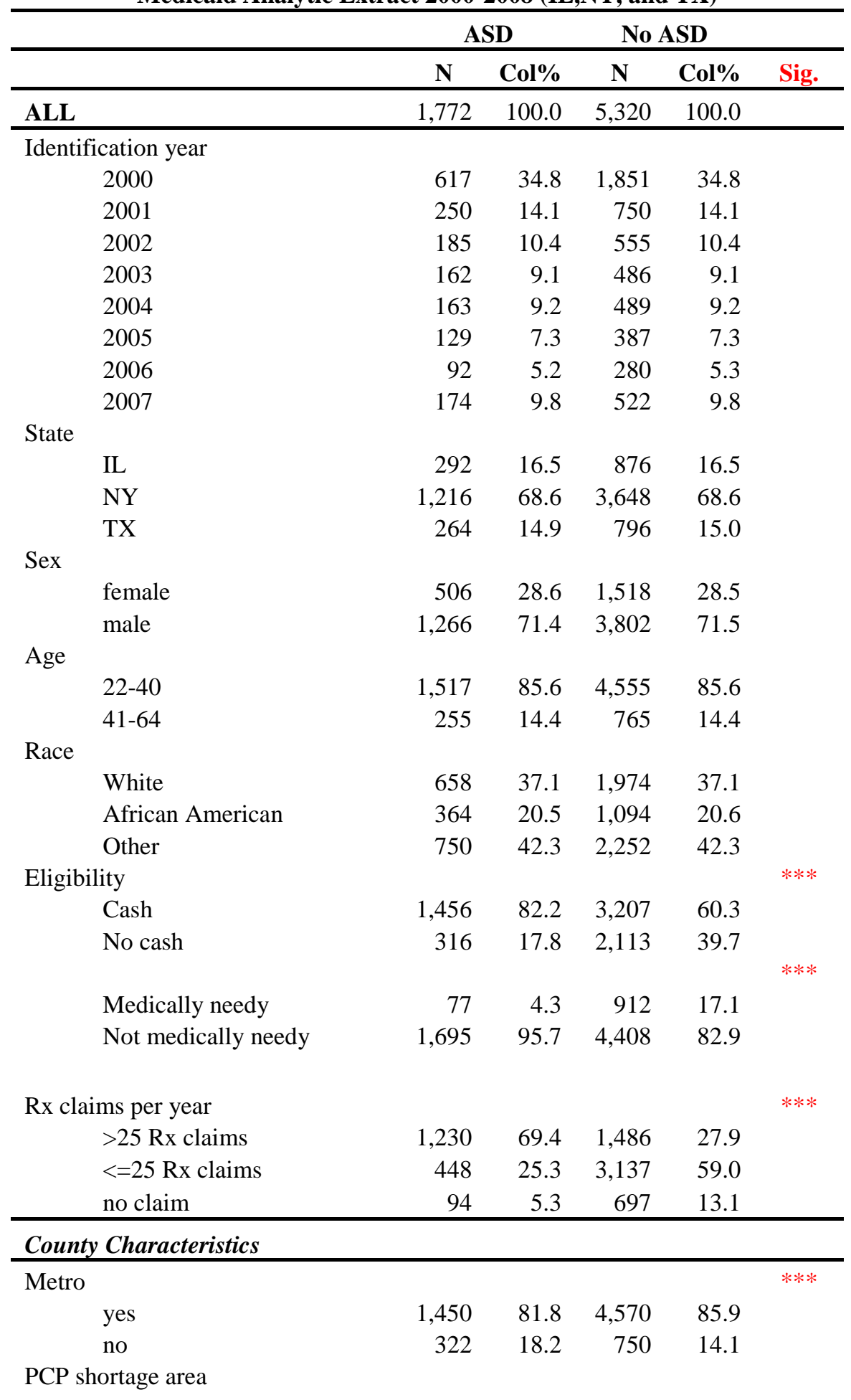


Table 1. Description of Sample by ASD diagnoses Adults with and without ASD matched on age, race, and gender $(n=7,092)$ Medicaid Analytic Extract 2000-2008 (IL,NY, and TX)

\begin{tabular}{|c|c|c|c|c|c|}
\hline & \multicolumn{2}{|c|}{ ASD } & \multicolumn{2}{|c|}{ No ASD } & \multirow[b]{2}{*}{ Sig. } \\
\hline & $\mathbf{N}$ & $\mathrm{Col} \%$ & $\mathbf{N}$ & Col\% & \\
\hline $\mathbf{A L L}$ & 1,772 & 100.0 & 5,320 & 100.0 & \\
\hline yes & 1,575 & 88.9 & 4,810 & 90.4 & \\
\hline no & 197 & 11.1 & 510 & 9.6 & \\
\hline Mental health specialist shortage area & & & & & $*$ \\
\hline yes & 1,434 & 80.9 & 4,419 & 83.1 & \\
\hline no & 338 & 19.1 & 901 & 16.9 & \\
\hline Psychiatrists density & & & & & $* *$ \\
\hline high & 1,322 & 74.6 & 4,138 & 77.8 & \\
\hline low & 450 & 25.4 & 1,182 & 22.2 & \\
\hline Median household income level & & & & & $* * *$ \\
\hline $\mathrm{q} 1$ & 286 & 16.1 & 440 & 8.3 & \\
\hline $\mathrm{q} 2$ & 69 & 3.9 & 438 & 8.2 & \\
\hline $\mathrm{q} 3$ & 338 & 19.1 & 1,053 & 19.8 & \\
\hline $\mathrm{q} 4$ & 1,079 & 60.9 & 3,389 & 63.7 & \\
\hline HS education and above & & & & & $* * *$ \\
\hline $\mathrm{q} 1$ & 1,071 & 60.4 & 3,164 & 59.5 & \\
\hline $\mathrm{q} 2$ & 80 & 4.5 & 483 & 9.1 & \\
\hline $\mathrm{q} 3$ & 244 & 13.8 & 737 & 13.9 & \\
\hline $\mathrm{q} 4$ & 377 & 21.3 & 936 & 17.6 & \\
\hline $\begin{array}{l}\text { Note: Based on chi-square estimates for } \\
\text { 22-64 years with no Medicare coverage } \\
\text { 2000-2008.ASD: Autism Spectrum Dis } \\
\text { Care Provider. Col } \% \text { : Column percenta } \\
* * * \mathrm{P}<0.001 ; * * 0.001 \leq \mathrm{P}<0.01 ; * 0.0\end{array}$ & $\begin{array}{l}\text { fee for } \\
\text { and ali } \\
\text { orders; } \\
\text { ges; ns } \\
1 \leq \mathrm{P}<\end{array}$ & $\begin{array}{l}\text { service } \\
\text { e in IL, } \\
\text { x: Presc } \\
\text { not sign }\end{array}$ & $\begin{array}{l}\text { rolled i } \\
\text { IY, and } \\
\text { iption D } \\
\text { icant at }\end{array}$ & $\begin{array}{l}\text { lividual } \\
\text { X Medi } \\
\text { ug; PCP } \\
<0.0516\end{array}$ & $\begin{array}{l}\text { aged } \\
\text { aid fro } \\
\text { Prima } \\
\text { el. Si }\end{array}$ \\
\hline
\end{tabular}


Table 2. Description of sample baseline characteristics by ASD diagnoses Adults with and without ASD matched on age, race, and gender ( $n=7,092)$ Medicaid Analytic Extract 2000-2008 (IL,NY, and TX)

\begin{tabular}{|c|c|c|c|c|c|c|}
\hline & & \multicolumn{2}{|c|}{ ASD } & \multicolumn{2}{|c|}{ No ASD } & \multirow[b]{2}{*}{ Sig. } \\
\hline & & $\mathbf{N}$ & $\mathrm{Col} \%$ & $\mathbf{N}$ & $\mathrm{Col} \%$ & \\
\hline ALL & & 1,772 & 100.0 & 5,320 & 100.0 & \\
\hline Psychiatric Comorbidity & & 1,440 & 81.3 & 2,216 & 41.7 & $* * *$ \\
\hline \multicolumn{7}{|l|}{ Adjustment disorders } \\
\hline & yes & 58 & 3.3 & 138 & 2.6 & \\
\hline & no & 1,714 & 96.7 & 5,182 & 97.4 & \\
\hline \multirow[t]{3}{*}{ Anxiety } & & & & & & $* * *$ \\
\hline & yes & 216 & 12.2 & 299 & 5.6 & \\
\hline & no & 1,556 & 87.8 & 5,021 & 94.4 & \\
\hline \multirow[t]{3}{*}{ ADD/ADHD } & & & & & & $* * *$ \\
\hline & yes & 146 & 8.2 & 37 & 0.7 & \\
\hline & no & 1,626 & 91.8 & 5,283 & 99.3 & \\
\hline \multirow[t]{3}{*}{ Intellectual disabilities } & & & & & & $* * *$ \\
\hline & yes & 1,231 & 69.5 & 270 & 5.1 & \\
\hline & no & 541 & 30.5 & 5,050 & 94.9 & \\
\hline \multicolumn{7}{|l|}{ Mood disorders } \\
\hline & yes & 256 & 14.4 & 839 & 15.8 & \\
\hline & no & 1,516 & 85.6 & 4,481 & 84.2 & \\
\hline \multicolumn{7}{|l|}{ Personality disorders } \\
\hline & yes & 44 & 2.5 & 105 & 2.0 & \\
\hline & no & 1,728 & 97.5 & 5,215 & 98.0 & \\
\hline \multirow[t]{3}{*}{ Schizophrenia } & & & & & & $* * *$ \\
\hline & yes & 294 & 16.6 & 590 & 11.1 & \\
\hline & no & 1,478 & 83.4 & 4,730 & 88.9 & \\
\hline \multicolumn{2}{|c|}{ Alcohol/Substance use disorders } & & & & & $* * *$ \\
\hline & yes & 36 & 2.0 & 948 & 17.8 & \\
\hline & no & 1,736 & 98.0 & 4,372 & 82.2 & \\
\hline \multicolumn{2}{|c|}{ Non-Psychiatric Comorbidity } & 708 & 40.0 & 2,429 & 45.7 & **** \\
\hline \multirow[t]{3}{*}{ Cancer } & & & & & & $* * *$ \\
\hline & yes & 21 & 1.2 & 149 & 2.8 & \\
\hline & no & 1,751 & 98.8 & 5,171 & 97.2 & \\
\hline \multirow[t]{3}{*}{ Gastrointestinal disorders } & & & & & & $* * *$ \\
\hline & yes & 223 & 12.6 & 583 & 11.0 & \\
\hline & no & 1,549 & 87.4 & 4,737 & 89.0 & \\
\hline \multirow[t]{3}{*}{ Respiratory disorders } & & & & & & $* * *$ \\
\hline & yes & 267 & 15.1 & 1,010 & 19.0 & \\
\hline & no & 1,505 & 84.9 & 4,310 & 81.0 & \\
\hline
\end{tabular}


Table 2. Description of sample baseline characteristics by ASD diagnoses Adults with and without ASD matched on age, race, and gender $(n=7,092)$ Medicaid Analytic Extract 2000-2008 (IL,NY, and TX)

\begin{tabular}{|c|c|c|c|c|c|c|}
\hline & & \multicolumn{2}{|c|}{ ASD } & \multicolumn{2}{|c|}{ No ASD } & \multirow[b]{2}{*}{ Sig. } \\
\hline & & $\mathbf{N}$ & Col\% & $\mathbf{N}$ & $\mathrm{Col} \%$ & \\
\hline $\mathbf{A L L}$ & & 1,772 & 100.0 & 5,320 & 100.0 & \\
\hline \multicolumn{3}{|c|}{ Cardiovascular/Cerebrovascular disorders } & & & & $* *$ \\
\hline & yes & 242 & 13.7 & 897 & 16.9 & \\
\hline & no & 1,530 & 86.3 & 4,423 & 83.1 & \\
\hline \multirow[t]{3}{*}{ Diabetes } & & & & & & $*$ \\
\hline & yes & 63 & 3.6 & 250 & 4.7 & \\
\hline & no & 1,709 & 96.4 & 5,070 & 95.3 & \\
\hline \multirow[t]{3}{*}{ Muscoskeletal disorders } & & & & & & $* * *$ \\
\hline & yes & 216 & 12.2 & 852 & 16.0 & \\
\hline & no & 1,556 & 87.8 & 4,468 & 84.0 & \\
\hline \multicolumn{7}{|l|}{ Other Health Conditions } \\
\hline \multirow[t]{3}{*}{ Epilepsy } & & & & & & $* * *$ \\
\hline & yes & 403 & 22.7 & 257 & 4.8 & \\
\hline & no & 1,369 & 77.3 & 5,063 & 95.2 & \\
\hline \multirow[t]{3}{*}{ Headache/Migraine } & & & & & & $* * *$ \\
\hline & yes & 18 & 1.0 & 176 & 3.3 & \\
\hline & no & 1,754 & 99.0 & 5,144 & 96.7 & \\
\hline \multirow[t]{3}{*}{ Thyroid } & & & & & & $* * *$ \\
\hline & yes & 83 & 4.7 & 71 & 1.3 & \\
\hline & no & 1,689 & 95.3 & 5,249 & 98.7 & \\
\hline \multirow[t]{3}{*}{ Other endocrine disorders } & & & & & & $* * *$ \\
\hline & yes & 192 & 10.8 & 214 & 4.0 & \\
\hline & no & 1,580 & 89.2 & 5,106 & 96.0 & \\
\hline \multicolumn{7}{|l|}{ Nutrition deficiencies } \\
\hline & yes & 15 & 0.8 & 42 & 0.8 & \\
\hline & no & 1,757 & 99.2 & 5,278 & 99.2 & \\
\hline \multirow[t]{3}{*}{ Lipid disorders } & & & & & & $* * *$ \\
\hline & yes & 84 & 4.7 & 131 & 2.5 & \\
\hline & no & 1,688 & 95.3 & 5,189 & 97.5 & \\
\hline \multicolumn{2}{|c|}{ Fluid and electrolyte dysfunctions } & & & & & $* * *$ \\
\hline & yes & 84 & 4.7 & 168 & 3.2 & \\
\hline & no & 1,688 & 95.3 & 5,152 & 96.8 & \\
\hline \multirow[t]{3}{*}{ Jaw and teeth disorders } & & & & & & $* * *$ \\
\hline & yes & 184 & 10.4 & 155 & 2.9 & \\
\hline & no & 1,588 & 89.6 & 5,165 & 97.1 & \\
\hline \multirow[t]{2}{*}{ Hematological disorders } & & & & & & $* * *$ \\
\hline & yes & 115 & 6.5 & 240 & 4.5 & \\
\hline
\end{tabular}


Table 2. Description of sample baseline characteristics by ASD diagnoses Adults with and without ASD matched on age, race, and gender $(n=7,092)$ Medicaid Analytic Extract 2000-2008 (IL,NY, and TX)

\begin{tabular}{|c|c|c|c|c|c|c|}
\hline & & \multicolumn{2}{|c|}{ ASD } & \multicolumn{2}{|c|}{ No ASD } & \multirow[b]{2}{*}{ Sig. } \\
\hline & & $\mathbf{N}$ & Col\% & $\mathbf{N}$ & Col\% & \\
\hline \multirow[t]{2}{*}{ ALL } & & 1,772 & 100.0 & 5,320 & 100.0 & \\
\hline & no & 1,657 & 93.5 & 5,080 & 95.5 & \\
\hline \multirow[t]{3}{*}{ Genito-urinary disorders } & & & & & & $* * *$ \\
\hline & yes & 168 & 9.5 & 423 & 8.0 & \\
\hline & no & 1,604 & 90.5 & 4,897 & 92.0 & \\
\hline \multirow[t]{3}{*}{ Paralysis } & & & & & & $* * *$ \\
\hline & yes & 101 & 5.7 & 157 & 3.0 & \\
\hline & no & 1,671 & 94.3 & 5,163 & 97.0 & \\
\hline \multirow[t]{3}{*}{ Infections } & & & & & & $* * *$ \\
\hline & yes & 381 & 21.5 & 924 & 17.4 & \\
\hline & no & 1,391 & 78.5 & 4,396 & 82.6 & \\
\hline \multirow[t]{3}{*}{ Skin disorders } & & & & & & $* * *$ \\
\hline & yes & 376 & 21.2 & 786 & 14.8 & \\
\hline & no & 1,396 & 78.8 & 4,534 & 85.2 & \\
\hline \multirow[t]{3}{*}{ Blindness/vision defects } & & & & & & $* *$ \\
\hline & yes & 96 & 5.4 & 197 & 3.7 & \\
\hline & no & 1,676 & 94.6 & 5,123 & 96.3 & \\
\hline \multirow[t]{3}{*}{ Hearing impairments } & & & & & & $* * *$ \\
\hline & yes & 328 & 18.5 & 210 & 3.9 & \\
\hline & no & 1,444 & 81.5 & 5,110 & 96.1 & \\
\hline
\end{tabular}

Note: Based on chi-square estimates for fee for service enrolled individuals aged 2264 years with no Medicare coverage and alive in IL, NY, and TX Medicaid from 2000-2008. ASD: Autism Spectrum Disorders; Col \%: Column percentages; ns: not significant at $\mathrm{p}<0.05$ level. Fischer's exact tests were run for variables with expected counts $<5$. Sig.: *** $\mathrm{P}<0.001 ; * * 0.001 \leq \mathrm{P}<0.01 ; * 0.01 \leq \mathrm{P}<0.05$. 
Table 3a. Healthcare utilization and expenditures by ASD diagnoses Mean, medians, and standard deviations for healthcare utilization and expenditures Adults with and without ASD matched on age, race, and gender $(n=7,092)$ Medicaid Analytic Extract 2000-2008 (IL,NY, and TX)

\begin{tabular}{|c|c|c|c|c|c|}
\hline & \multicolumn{2}{|l|}{ ASD } & \multicolumn{2}{|c|}{ No ASD } & \multirow[t]{2}{*}{ Sig. } \\
\hline & Mean, Median & SD & Mean, Median & SD & \\
\hline \multicolumn{6}{|c|}{$\begin{array}{l}\text { Healthcare utilization (number of } \\
\text { visits, claims in follow up year) }\end{array}$} \\
\hline OT & 32,12 & 49 & 8,3 & 17 & $* * *$ \\
\hline IP & $0.30,0$ & 1 & $0.46,0$ & 2 & $* * *$ \\
\hline $\mathbf{R x}$ & 51,45 & 38 & 24,12 & 32 & $* * *$ \\
\hline ER & 1,0 & 7 & 1,0 & 3 & \\
\hline \multicolumn{6}{|c|}{ Healthcare expenditures (2008 constant dollars) } \\
\hline OT & $\$ 4,375, \$ 1,092$ & $\$ 7,836$ & $\$ 854, \$ 187$ & $\$ 2,751$ & $* * *$ \\
\hline IP & $\$ 3,258, \$ 0$ & $\$ 14,130$ & $\$ 4,562, \$ 0$ & $\$ 20,368$ & $*$ \\
\hline $\mathbf{R x}$ & $\$ 6,067, \$ 4,925$ & $\$ 5,390$ & $\$ 3,144, \$ 625$ & $\$ 6,798$ & $* * *$ \\
\hline ER & $\$ 15,929, \$ 0$ & $\$ 38,747$ & $\$ 2,598, \$ 0$ & $\$ 13,133$ & $* * *$ \\
\hline Total & $\$ 13,700, \$ 9,072$ & $\$ 17,095$ & $\$ 8,560, \$ 1,760$ & $\$ 22,534$ & $* * *$ \\
\hline
\end{tabular}

Note: Based on t-tests estimates for fee for service enrolled individuals aged 22-64 years with no Medicare coverage and alive in IL, NY, and TX Medicaid from 2000-2008.

ASD: Autism Spectrum Disorders; Rx: Prescription Drug; OT: Outpatient Visits; IP: Inpatient Hospitalizations; ER: Emergency Room

Total expenditures are sum of OT, IP, and Rx expenditures

Sig.: ***P $<0.001 ; * * 0.001 \leq \mathrm{P}<0.01 ; * 0.01 \leq \mathrm{P}<0.05$. 
Table 3b. Healthcare utilization and expenditures by ASD diagnoses among users Mean, medians, and standard deviations for healthcare utilization and expenditures Adults with and without ASD matched on age, race, and gender $(n=7,092)$ Medicaid Analytic Extract 2000-2008 (IL,NY, and TX)

\begin{tabular}{|c|c|c|c|c|c|c|}
\hline & \multirow[t]{2}{*}{$\mathbf{N}$} & \multicolumn{2}{|l|}{ ASD } & \multicolumn{2}{|c|}{ No ASD } & \multirow[b]{2}{*}{ Sig. } \\
\hline & & Mean, Median & SD & Mean, Median & SD & \\
\hline \multicolumn{7}{|c|}{$\begin{array}{l}\text { Healthcare utilization } \\
\text { (number of visits, claims in } \\
\text { follow up year) }\end{array}$} \\
\hline OT & 5,969 & 34,13 & 49 & 10,5 & 18.0 & $* * *$ \\
\hline IP & 1,313 & 2,1 & 2 & 2,1 & 3.0 & \\
\hline $\mathbf{R x}$ & 6,313 & 54,48 & 37 & 28,16 & 33.0 & $* * *$ \\
\hline $\mathbf{E R}$ & 2,741 & 3,2 & 11 & 3,2 & 5.0 & \\
\hline ALOS & 1,313 & 10,6 & 17 & 8,4 & 14.0 & $*$ \\
\hline \multicolumn{7}{|c|}{ Healthcare expenditures (2008 constant dollars) } \\
\hline OT & 5,969 & $\$ 4,617, \$ 1,272$ & $\$ 7,980$ & $\$ 1,059, \$ 303$ & $\$ 3,028$ & $* * *$ \\
\hline IP & 1,313 & $\$ 22,912, \$ 11,812$ & $\$ 30,928$ & $\$ 22,872, \$ 10,199$ & $\$ 40,773$ & \\
\hline $\mathbf{R x}$ & 6,313 & $\$ 6,388, \$ 5,298$ & $\$ 5,342$ & $\$ 3,613, \$ 968$ & $\$ 7,170$ & $* * *$ \\
\hline ER & 2,741 & $\$ 47,438, \$ 15,967$ & $\$ 54,578$ & $\$ 6,441, \$ 1,937$ & $\$ 20,073$ & $* * *$ \\
\hline
\end{tabular}

Note: Based on t-tests estimates for fee for service enrolled individuals aged 22-64 years with no Medicare coverage and alive in IL, NY, and TX Medicaid from 2000-2008. Users represent only those individuals who used respective services.

ASD: Autism Spectrum Disorders; Rx: Prescription Drug; OT: Outpatient Visits; IP: Inpatient Hospitalizations; ER: Emergency Room; ALOS: Average Length of Stay

Sig.: ***P $<0.001 ; * * 0.001 \leq \mathrm{P}<0.01 ; * 0.01 \leq \mathrm{P}<0.05$. 
PhD Dissertation Chapter 2

Table 4. Odds Ratios, 95\% Confidence Intervals, Parameter Estimates, and Standard Errors for Healthcare Utilization and Expenditures Adults with and without ASD matched on age, race, and gender $(n=7,092)$

Medicaid Analytic Extract 2000-2008 (IL,NY, and TX)

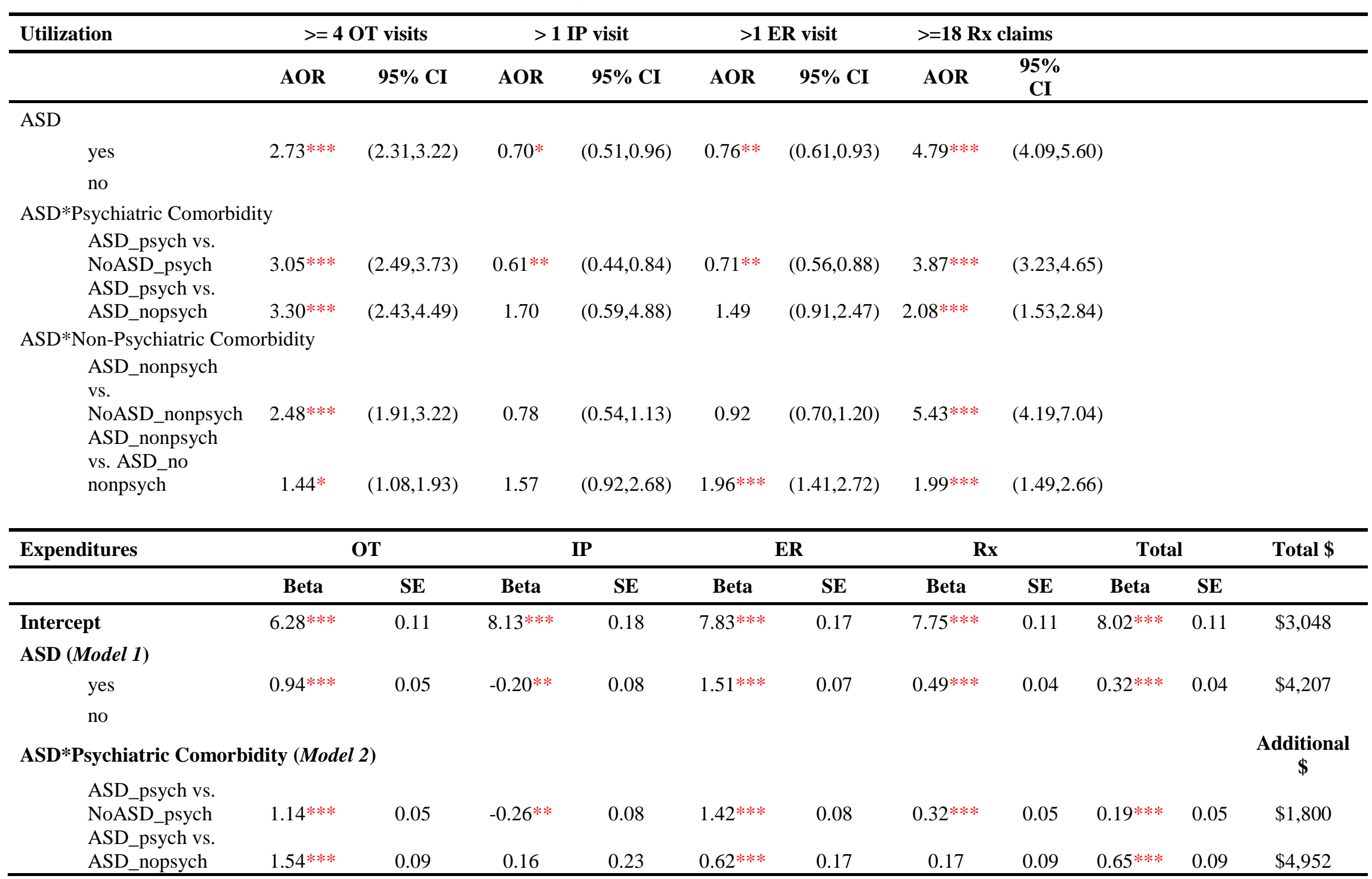


Table 4. Odds Ratios, 95\% Confidence Intervals, Parameter Estimates, and Standard Errors for Healthcare Utilization and Expenditures Adults with and without ASD matched on age, race, and gender $(n=7,092)$

Medicaid Analytic Extract 2000-2008 (IL,NY, and TX)

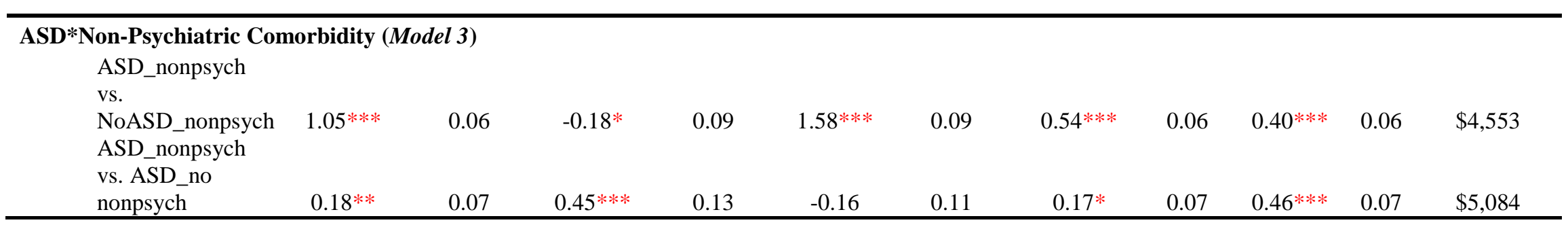

Note: Based on estimates for fee for service enrolled individuals aged 22-64 years with no Medicare coverage and alive in IL, NY, and TX Medicaid from 20002008.ASD: Autism Spectrum Disorders; Rx: Prescription Drug; OT: Outpatient Visits; IP: Inpatient Hospitalizations; ER: Emergency Room.

AOR's represent estimates from multinomial logistic regression analyses respectively after adjusting for cohort (before/after 2004), state, eligibility, county

characteristics (metro status, median household income, above high school education, PCP shortage area, mental health specialist shortage area, and psychiatrist density), baseline Rx use, psychiatric comorbidity, and non-psychiatric comorbidity.

Parameter estimates represent results from generalized linear models with gamma distribution and log-link function after adjusting for cohort (before/after 2004), state, eligibility, county characteristics (metro status, median household income, above high school education, PCP shortage area, mental health specialist shortage area, and psychiatrist density), baseline Rx use, psychiatric comorbidity, and non-psychiatric comorbidity.

Model for IP expenditures was also adjusted for average length of stay.

Model for Rx and total expenditures was not adjusted for baseline prescription drug use due to high collinearity issues.

Total expenditures represents sum of OT, IP, and Rx expenditures.

Model 1, 2, and 3 represent separate regressions.

Total dollar $=$ exponentiated (beta)

Additional Dollar $=$ exponentiated (intercept) - exponentiated (beta) for each regression.

Sig.: ***P $<0.001 ; * * 0.001 \leq \mathrm{P}<0.01 ; * 0.01 \leq \mathrm{P}<0.05$ 
PhD Dissertation Chapter 3

\title{
Study 2. The Burden of Emergency Department (ED) Use among Adults with and without ASD
}

\author{
Prepared by Rini Vohra \\ For Submission to PhD Dissertation Committee Members \\ Dr. Suresh Madhavan \\ Dr. Usha Sambamoorthi \\ Dr. Nilanjana Dwibedi \\ Dr. Claire StPeter \\ Dr. Susannah Poe
}




\section{PhD Dissertation Chapter 3}

\section{CONTENTS}

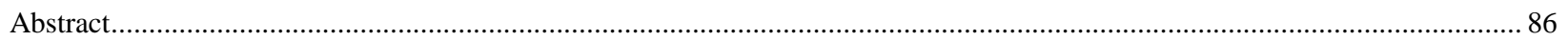

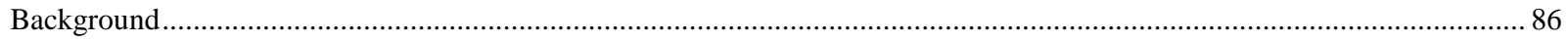

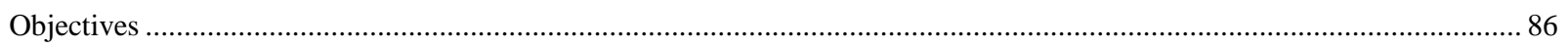

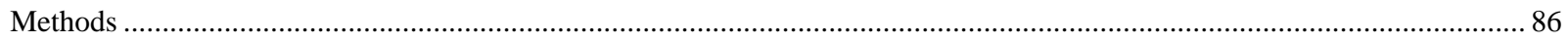

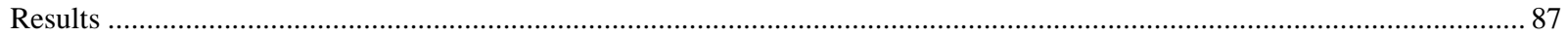

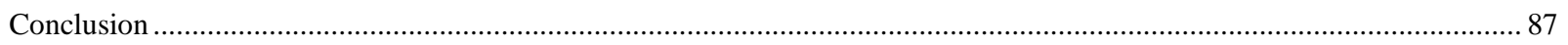

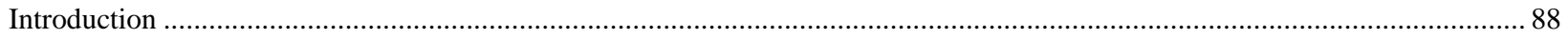

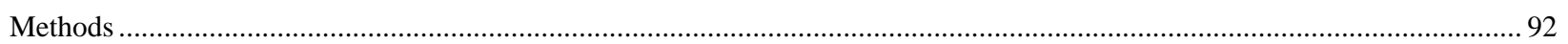

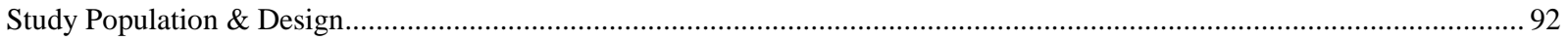

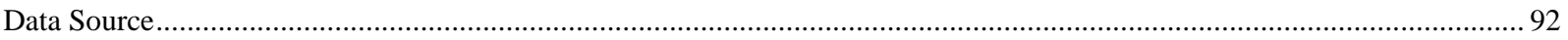

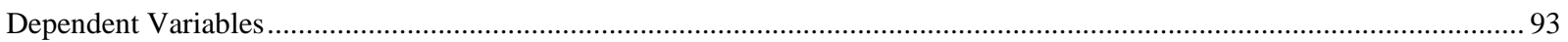

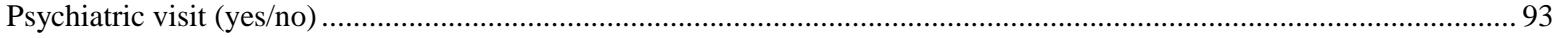

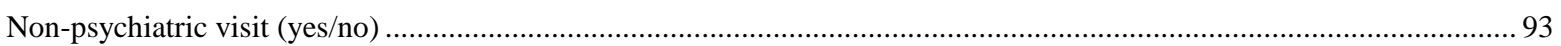

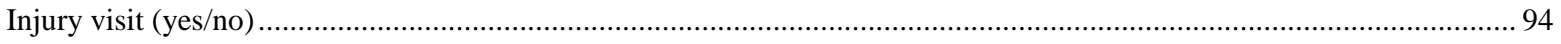

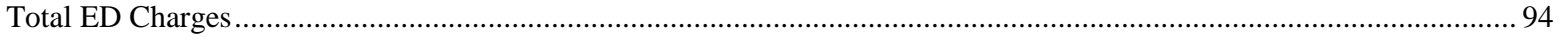

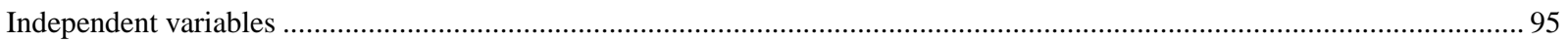

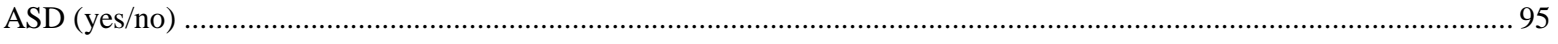

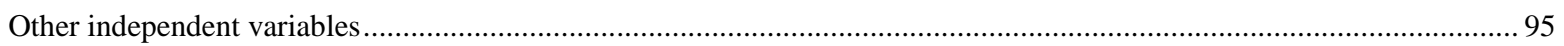

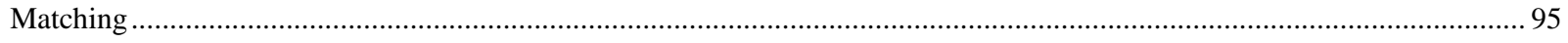

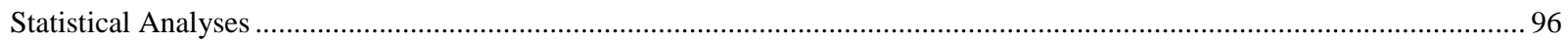

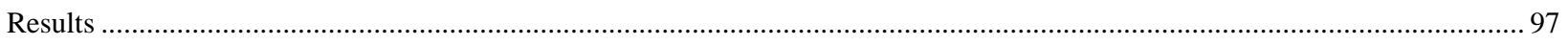

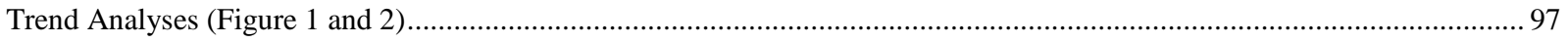

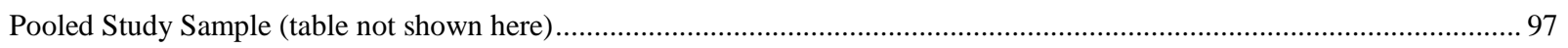

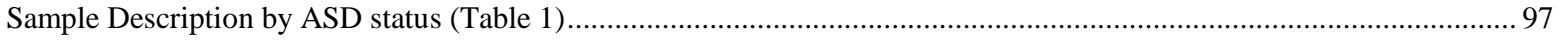

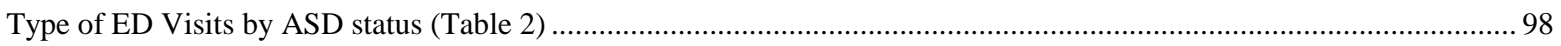

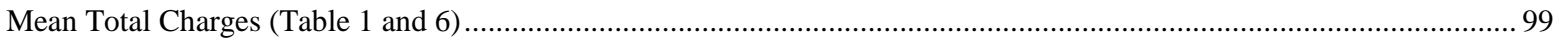

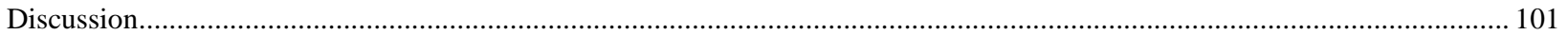

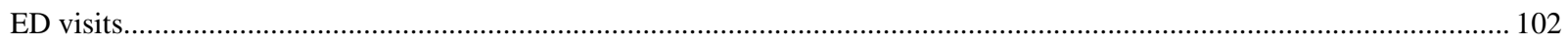

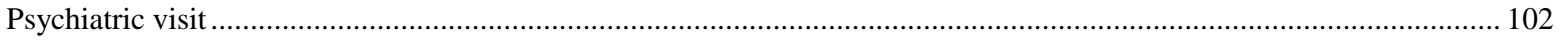

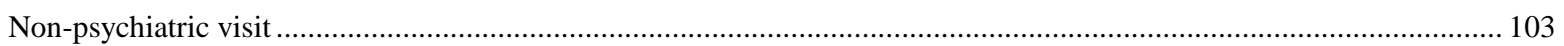

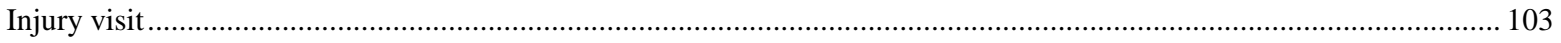

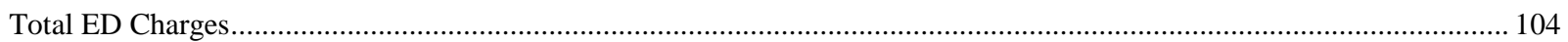

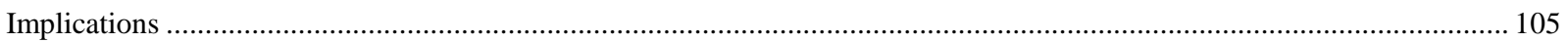

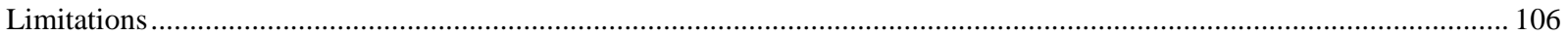

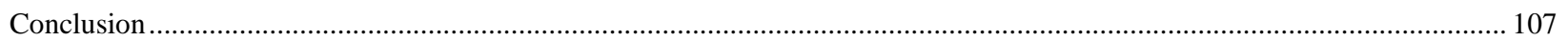

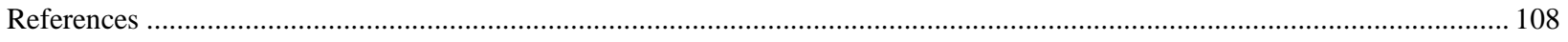

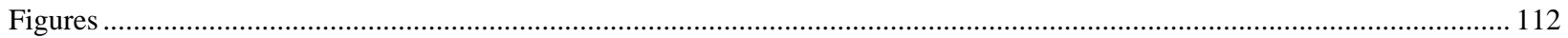

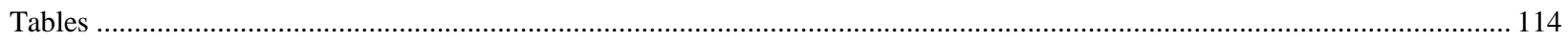




\title{
PhD Dissertation Chapter 3
}

\author{
Abstract \\ Background: The use of emergency department (ED) visits among adults with psychiatric needs \\ is known to be very high, yet there are no comprehensive studies that have characterized ED use \\ among adults with autism spectrum disorders (ASD). Due to the lack of clarity on adult ASD \\ treatment guidelines and possible delayed/foregone care in outpatient settings, adults with ASD \\ may be at a substantial risk of high ED use and costs.
}

Objectives: 1) To examine the trends in ED visits and mean total ED charges for adults with ASD over a period of 6 years; 2) To examine and compare the types of ED use (psychiatric, nonpsychiatric, and injury) among adults with and without ASD.

Methods: A cross-sectional study using discharge level data from the Nationwide Emergency Department Sample (NEDS; 2006-2011) was conducted to the examine trends and characteristics of ED visits among adults aged 22-64 years, with and without ASD (ICD9-CM code: 299.xx). ASD cases were matched to no ASD controls by age and gender using propensity score matching (1:3). Trends are presented as annual weighted rates and mean total charges for all, psychiatric (principal diagnosis of a psychiatric disorder), non-psychiatric (principal diagnosis of a non-psychiatric disorder), and injury (any) visits among adults with ASD. Chi square analyses and t-tests were conducted to examine sub group differences in sociodemographic, hospital, and patient disposition characteristics across adults with and without ASD. Weighted rates of psychiatric, non-psychiatric, and injury visits were compared between adults with and without ASD. Multivariate adjusted logistic regressions were run to examine the likelihood of a psychiatric, non-psychiatric, and an injury visit among adults with ASD as compared to adults without ASD. All analyses adjusted for the NEDS complex survey design. 


\section{PhD Dissertation Chapter 3}

Results: Around $0.4 \%(\mathrm{n}=25,527)$ of ED visits were associated with an adult ASD diagnosis in the unmatched sample. The rates of ED visits among adults with ASD more than doubled from 2006 to 2011 (10,047 to 23,992 per 100,000 admissions). Around one-third of the ED visits among adults with ASD led to an inpatient hospitalization. The rates of specific ED visits among adults with and without ASD were: psychiatric (15\% ASD vs. $\left.4.2 \%_{\text {noASD }}\right)$, non-psychiatric $\left(16 \%_{\text {ASD }}\right.$ vs. $\left.14 \%_{\text {noASD }}\right)$, and injury ( $24 \%_{\text {ASD }}$ vs. $\left.28 \%_{\text {noASD }}\right)$. Most common ED visits associated with adults with ASD were: epilepsy (8.8\%), falls (6.5\%), schizophrenia (3.8\%), and respiratory disorders (3.8\%). Adults with ASD were found to be more likely to have a psychiatric visit $(\mathrm{AOR}=2.63,95 \% \mathrm{CI}=2.41-2.88)$, a non-psychiatric visit $(\mathrm{AOR}=1.07,95 \% \mathrm{CI}=1.01-1.14)$, and an injury visit $(\mathrm{AOR}=1.10,95 \% \mathrm{CI}=1.04-1.16)$ as compared to adults without ASD. Mean total ED charges for adults with ASD were 2.3 times higher than adults without ASD.

Conclusion: Rates of ED visits among adults with ASD are on the rise. Adults with ASD use ED for different reasons which are not restricted to psychiatric needs, but also extend to nonpsychiatric needs and injuries. ED visits among adults with ASD are also associated with significantly greater hospitalization rates as compared to adults without ASD. Mean total ED charges for adults with ASD are almost twice as high as charges for adults without ASD. Prevalence, risk factors, and burden of ED utilization among adults with ASD is understudied and future studies should examine the reasons and impact of such ED utilization on long term healthcare costs. 


\section{PhD Dissertation Chapter 3}

\section{Introduction}

The burden of emergency department (ED) use in the US is high and the total number of national ED visits in the year 2005 ranged from 109.2 million to 116.3 million (Owens et al., 2010; Tang, Stein, Hsia, Maselli, \& Gonzales, 2010). According to a report by (Choudhry et al., 2007) on association of community affiliated plans, at least one-third of ED visits were avoidable/non-urgent/ambulatory care sensitive and treatable in primary care settings. Surprisingly, over $\$ 18$ billion dollars are spent annually on such avoidable ED visits in the US (Choudhry et al., 2007). Since ED is often the most critical point of healthcare delivery and for many individuals the only point of healthcare delivery, it is important to document the extent of ED utilization among patients with high healthcare needs and among those who are at high risk of frequent returns.

Even though adults with ASD have considerable healthcare needs (Billstedt et al., 2005; Magiati et al., 2014), there is a lack of comprehensive documentation of characteristics and patterns of ED use among this group. Previous studies on use of ED services among individuals with ASD have been either restricted to pediatric/adolescent population (Croen et al., 2006; Kalb et al., 2012; Wharff, Ginnis, Ross, \& Blood, 2011) and/or examination of ED use as a part of a bigger study with no specific details on types of ED visits (Croen et al., 2006; Kato et al., 2013; Nicolaidis et al., 2013). Nonetheless, such studies have provided insights which corroborate the hypothesis that adults with ASD are at high risk of ED use. Nicolaidis et al. (2013) examined the healthcare experiences of adults with and without ASD via an online cross sectional survey and found that adults with ASD had greater odds of using an ED as compared to adults without ASD $(\mathrm{OR}=2.1,95 \% \mathrm{CI}=1.8-3.8)$. (Kato et al., 2013) compared the characteristics of suicide attempts in a hospital emergency room between adults with and without ASD. The authors found that about $7.3 \%$ of patients with suicidal emergencies had ASD, and those with ASD had a 


\section{PhD Dissertation Chapter 3}

significantly greater prevalence of adjustment disorders (70\%) and a longer length of stay in the intensive care unit/hospital as compared to adults without ASD. The authors suggested that patients with ASD, due to impulsive behavior and aggression tendencies may be more likely to choose lethal methods of suicide, and may even be more likely to succeed in their first suicidal attempt in cases where stressors are not spontaneous, and when there is a lack of psychiatric treatment in the recent past. Despite such studies, there is a need to characterize preponderant ED visits among adults with ASD and compare them to adults without ASD because the former has various concerns that puts them at a greater risk of worse ED outcomes:

\section{Comorbidities}

Adults with ASD have extensive comorbidities such as intellectual disabilities, depression, anxiety, ADHD, and substance dependence disorder (Hofvander et al., 2009b; Lugnegard et al., 2011b) along with core autistic symptoms that present greater challenges in care as compared to adults without ASD (Pines et al., 2011). A study by (E. R. Williams et al., 2001) found high prevalence of anxiety and depressive disorders among frequent attenders of ED. Substance abuse also influences ED use over a period of time for adults with other psychiatric illness $(\mathrm{OR}=4.9,95 \% \mathrm{CI}=3.5-6.9)(\mathrm{G}$. M. Curran et al., 2003). In addition, some studies have shown that many patients (as high as 24\%) with depressive disorders (comorbid or not) present to the ED for traumatic physical injuries/self-inflicted injuries (Doshi, Boudreaux, Wang, Pelletier, \& Camargo, 2005; Richmond et al., 2007). This indicates that adults with ASD may have a higher risk of injuries not only because of their own behavioral tendencies but also because of high prevalence of comorbid depression (Lugnegard et al., 2011a; Lunsky et al., 2009). A very recent study by Croen and colleagues (Croen et al., 2015) showed that adults with ASD are also more likely to have greater prevalence of non-psychiatric comorbidities such as 


\section{PhD Dissertation Chapter 3}

diabetes, cardiovascular conditions, and high cholesterol, which may also be contribute to an increase in ED use and worsen ED experiences for adults with ASD.

\section{Disruptive Behaviors and Injuries}

Individuals with ASD are affected by challenging behaviors such as aggression, destruction, self-inflicted injuries, and other disruptive behaviors (Matson \& Rivet, 2008) that many a time require emergent care. (Doshi et al., 2005) used the National Hospital Ambulatory Medical Care Survey (NHAMCS) to examine the ED visit patterns for attempted suicide and self-inflicted injuries which accounted for more than 400,000 ED visits per year $(0.4 \%$ of all ED visits). The most common cases in the ED were treated for poisoning ( $28 \%$ by unspecified drugs or medicinal substances, $27 \%$ tranquilizers and other psychotropic agents and $25 \%$ by analgesics/antipyretics/anti-rheumatics) followed by cutting or piercing. Knowing that individuals with ASD frequently engage in disruptive behaviors (Matson \& Rivet, 2008), it is very likely that they may present to ED with extreme self-inflicted injuries. Since, some adults with ASD have communication difficulties, providing care in ED for injuries, may also be a bigger challenge for ED primary care providers.

\section{Paucity of Trained Professionals}

Lack of trained psychiatrists and other mental health professionals that can manage individual ASD issues in specialty or primary care settings may also lead to greater ED use. (E. R. Williams et al., 2001) found that individuals with severe mental illness such as schizophrenia, bipolar disorder, and other psychosis who were frequent attenders of ED lacked a coordinated community program involving mental health, primary care, and ED. In addition, it was found that ED visits related to mental health, in many cases, could have been handled well in an 


\section{PhD Dissertation Chapter 3}

outpatient primary setting due to the non-urgent nature of the complaint (Sills \& Bland, 2002;

Soto et al., 2009). Thus, sometimes an ED becomes a source of primary care for those with psychiatric needs such as ASD due to the lack of access to mental health facilities in the community and lack of trained professionals who are able to provide ASD care (Bruder et al., 2012).

The current study aims to address the gaps in literature on ED utilization among adults with ASD. The study compares the trends in ED rates for adults with ASD across a span of six years using the Nationwide Emergency Database Sample (NEDS) 2006-2011 (Healthcare Cost and Utilization Project (HCUP), 2011). We also determine the differences in rates of specific ED visits such as psychiatric, non-psychiatric, and injury visits across adults with and without ASD. Examination of ED use will help highlight the deficits (if any) in quality of care/care coordination for adults with ASD provided in the primary care setting that possibly contributes to greater ED use and inpatient hospitalizations (Soto et al., 2009; E. R. Williams et al., 2001). Due to the rise in adult ASD cases (Brugha et al., 2011) and with greater number of individuals receiving ASD diagnoses than ever before, we hypothesized that rates of ED visits with ASD diagnosis will increase over the six year period. In addition, we also expected that adults with ASD will be more likely to have psychiatric, non-psychiatric, as well as an injury visit as compared to adults without ASD. 


\section{PhD Dissertation Chapter 3}

\section{Methods}

Study Population \& Design

Any ED visits among adults aged 22-64 years was the study population. A cross-sectional matched case-control design was used. Trend analyses was conducted with each year selected as a distinct data point in the study (2006-2011). For objectives other than trends, a pooled sample was used. If any variable had missingness $\geq 0.5 \%$, we created a missing indicator to account for any differences caused by missingness in our major independent variables.

\section{Data Source}

We used the Nationwide Emergency Database Sample (NEDS) 2006- 2011, largest all payer ED database including national estimates for hospital based ED data visits created for the Healthcare Cost and Utilization Project, Agency of Healthcare and Research Quality (Healthcare Cost and Utilization Project (HCUP), 2011). NEDS compiles discharge data collected from hospital billing records from both state emergency department databases (SEDD) and state inpatient databases (SID). The SID contains information on patients initially seen in the ED and then admitted to the same hospital. The SEDD capture information on ED visits that do not result in an admission (i.e., treat-and-release visits and transfers to another hospital).

The NEDS is built using a $20 \%$ stratified sample of institutions and collects data from 951 hospitals located in 30 states with an unweighted 30 million discharges each year. The NEDS presents rich information on type of ED visits (psychiatric, injury, etc.) along with up to 15 diagnoses associated with each visit, geographic information, hospital characteristics, and total charges for each ED visit. We expect that high proportion of adults with ASD will present to the ED, utilize ED services frequently, and are possibly associated with an increased risk of subsequent hospitalizations as compared to adults without ASD. Using a database such as NEDS 


\section{PhD Dissertation Chapter 3}

not only helps understand the extent and patterns of ED use among adults with ASD but also provides additional information on reasons for these visits such as type of injuries (which has not been previously reported in any study on adults with ASD), primary diagnosis for the ED visit, and the outcome(s) of the ED visits (e.g. treated and released, transfer to home health, or inpatient hospitalizations etc.).

\section{Dependent Variables}

Psychiatric visit (yes/no)

A psychiatric visit was identified by an ED visit with a principal diagnosis of a psychiatry disorder, other than ASD. The NEDS includes the single level clinical classification software (CCS) provided by Agency for Healthcare Research and Quality (Healthcare Cost and Utilization Project (HCUP), 2011) (https://www.hcup

us.ahrq.gov/toolssoftware/CCS/AppendixASingleDX.txt) to categorize diagnosis and procedure codes to clinically meaningful categories. We used nine broad categories of psychiatric disorders: adjustment disorders (CCS code: 650), alcohol use disorders (AUD; CCS code: 660), anxiety disorders (CCS code: 651), attention deficit disorders \& conduct behavior disorders (ADD; CCS code: 652), developmental disorders (CCS code: 654), mood disorders including depressive disorders and bipolar disorder (CCS code: 6571,6572), personality disorders (CCS code: 658), schizophrenia \& other psychotic disorders (CCS code: 659), and substance use disorders (SUD; CCS code: 661).

Non-psychiatric visit (yes/no)

A non-psychiatric visit was identified as an ED visit with a principal diagnosis of a nonpsychiatric disorder. We used six broad categories of non-psychiatric disorders some of which have been found to be very common among adults with ASD in a recent study (Croen et al., 2015): cancer (CCS code: 11-37, 39, 40-43), cardiovascular disease (CCS code: 100, 101, 105, 


\section{PhD Dissertation Chapter 3}

106, 108, 53, 98, 99, 109, 110-112), diabetes (CCS code: 49, 50), epilepsy (CCS code: 83), gastrointestinal disease (CCS code: 138-140, 141), and respiratory disease (CCS code: 125-128, 132-134).

Injury visit (yes/no)

NEDS 2006-2009 reported external causes of injuries in form of CCS ecodes (4 possible ecodes on each record), but 2009 onwards the NEDS reported injuries as a separate variable (injury on principal diagnosis, injury on other diagnoses, and no injury). Each type of injury was identified using CCS ecodes/ICD-9-CM codes prior to 2009 and by an injury variable after the year 2009 to create a single binary indicator for any injury (yes/no). Any visit with a record of the following codes qualified as an injury visit: cutting (CCS ecode: 2601), drowning (CCS ecode: 2602), fall (CCS ecode: 2603), fire (CCS ecode: 2604), machinery (CCS ecode: 2606), poison (CCS ecode: 2613), struck (struck by lightning or an object; CCS ecode: 2614), and suffocation (CCS ecode: 2615). Additional critical injury characteristics such as assault (by intent; yes/no), self-harm (by intent; CCS ecode: 662 excluding V6284), and suicidal ideation (ICD9-CM code: V6284) are also included in the study. We also report the severity of injury associated with an ED visit by examining presence of multiple cause of injuries (none or one, 2 or more), which indicates the total number of external cause of injury ecodes (valid and invalid).

\section{Total ED Charges}

The edited total charges for ED services associated with each visit was used to identify economic burden of ED visits. Total ED charges included both "treat and release" ED visits as well as ED visits that led to a hospitalization in the same hospital. The total ED charges were expressed in constant dollars to adjust for inflation over the period of six years. "Medical care services" part of the annual consumer price index (CPI) was utilized to transform/convert total 


\section{PhD Dissertation Chapter 3}

charges to 2011 constant dollars. The CPI was obtained from the Bureau of Labor Statistics (Bureau of Labor Statistics, US Department of Labor, 2014).

\section{Independent variables}

ASD (yes/no)

The NEDS provides up to 15 possible diagnoses recorded on each ED visit. Adults with ASD were identified using an ICD-9-CM diagnosis code in any position for: 299.xx (which includes autistic disorder, Asperger's syndrome, and other pervasive developmental disorders).

Other independent variables

Age (22-40, 41-54, and 55-64) and gender (male and female) were included as demographic characteristics. The socio-economic status was described using median household income for patient's zip code which was assigned as quartiles by the HCUP for each year (For e.g. in the 2011 NEDS, the median income was divided into: $\$ 1$ - $\$ 38,999, \$ 39,000$ - $\$ 47,999$, $\$ 48,000$ - $\$ 63,999$, and $\$ 64,000$ or more). We used the median household income for patient's zip code and categorized it into four major quartiles (Q1, Q2, Q3, Q4, and missing). Health insurance coverage selected as a primary payer for the ED visit was categorized into: public (Medicare/Medicaid), private, self-pay/other/no charge. Hospital characteristics included region (Northeast, Midwest, South, and West) and hospital location (rural, urban, and missing). Patient disposition characteristics included the type of ED event (treat and release, inpatient admission, transfer to another short term hospital/home health, and died/other). A mortality event was defined as any record of patient death reported on the ED visit (yes/no).

\section{Matching}

The ASD cases (1) and no ASD controls (3) were matched by age and gender using propensity score matching method with GREEDY algorithm. Predicted probabilities from a 


\section{PhD Dissertation Chapter 3}

multivariate logistic regression analysis on ASD status (yes, no) were used to identify and match the "nearest neighbor" with an ASD to a visit with no ASD.

\section{Statistical Analyses}

We conducted two sample chi-square tests to present trends in ED use and multivariate logistic and linear regressions to assess the significance of trends. The weighted rates (number per 100,000 admissions) are presented in form of graphs. Weighted rates for adults with ASD were calculated using number of ED visits with any ASD diagnosis (numerator) and total number of ED visits in the sample (denominator). Weighted rates for specific ED visits, for example psychiatric visits were calculated using number of psychiatric ED visits with any ASD diagnoses (numerator) and total number of psychiatric ED visits in the sample (denominator). For examining sub-group differences across adults with and without ASD in the pooled sample, bivariate analyses were conducted (Chi-square for categorical and t-tests for continuous variables). Numbers and weighted percentages are reported for each type of visit by ASD status. Unadjusted and multivariate adjusted logistic regressions were run for binary dependent variables (psychiatric, non-psychiatric, and injury visits). All procedures accounted for NEDS complex survey design. For all analyses, SAS v9.4 was used. 


\section{PhD Dissertation Chapter 3}

\section{Results}

\section{Trend Analyses (Figure 1 and 2)}

Rates of ED visits for adults with ASD (Figure 1) increased from 10,047 to 23,992 per every 100,000 ED visits from the years 2006 to 2011. Psychiatric visit rates for adults with ASD exhibited the steepest rise from 9,576 to 24,948 per every 100,000 psychiatric visits as compared to all other specific ED visits. Despite the actual rise in rates for all, psychiatric, non-psychiatric, and injury visits, none of the trends were significantly different from trends for adults without ASD. Figure 2 shows the change in mean total charges for all, psychiatric, non-psychiatric, and injury visits with ASD over a period of six years. The mean total ED charges were found to decrease significantly among adults with ASD for all other types of ED visits, except for psychiatric visits $(\mathrm{p}>0.05)$. However, the decline in mean total charges over the 6 year period was not very substantial $(0.02 \%, 0.21 \%$, and $0.18 \%$ for all, injury, and non-psychiatric visits respectively).

\section{Pooled Study Sample (table not shown here)}

In the pooled study sample 25,257 ED visits were among adults with ASD (0.4\% in unmatched sample). The ED visits were primarily among male gender (67\%) and age group 2240 years $(72 \%)$. Most of the ED visits in the sample were treat and release $(86 \%)$ and $0.3 \%$ visits were associated with a mortality event. Most common ED visits in the pooled sample were associated with an injury $(\mathrm{n}=27,193,26 \%)$, followed by a non-psychiatric disorder $(\mathrm{n}=14,574$, $14 \%)$, and a psychiatry disorder $(\mathrm{n}=7,005,7 \%)$.

Sample Description by ASD status (Table 1)

Around $80 \%$ of adults with ASD had public health insurance as a primary payer as compared to only $26 \%$ adults without ASD. One-third of ED visits among adults with ASD led to an inpatient admission (34\%) as compared to one-tenth of ED visits among adults without 


\section{PhD Dissertation Chapter 3}

ASD. Approximately one percent of adults with ASD had a mortality event after an ED visit as compared to $0.3 \%$ adults without ASD.

Type of ED Visits by ASD status (Table 2)

Fifteen percent of adults with ASD had a psychiatric visit as compared to $4.2 \%$ adults without ASD. Proportion of adults with ASD with an injury visit was significantly less as compared to adults without ASD (23.7\% ASD vs. 27.7\% NoASD). However, non-psychiatric visits (16.1\%) were much more common among adults with ASD as compared to adults without ASD (13.6\%). Within psychiatric visits, a majority of adults with ASD came to ED with a principal diagnosis of: schizophrenia (3.8\%), followed by bipolar disorder (3.2\%), depression (2.5\%), and intellectual disabilities (1.6\%). Even though the rates of injury visits were much lower among adults with ASD as compared to adults without ASD, there were certain injuries that were more common in the former group. Adults with ASD had higher rates of injuries due to falls $(6.5 \%$ ASD vs. $\left.5.0 \%_{\text {noASD }}\right)$, poisoning (1.4\% ASD vs. $\left.0.8 \%_{\text {noASD }}\right)$, self-harm ( $1.9 \%_{\text {ASD }}$ vs. $0.5 \%$ noASD $)$, and suicidal ideation (2.6\% ASD vs. $0.9 \%$ noASD). Adults with ASD also had significantly greater rates of non-psychiatric visits with a principal diagnosis of cancer ( $0.3 \%$ ASD vs. $0.2 \%$ noASD $)$, diabetes (1.1\% ASD vs. $0.8 \%$ noASD), and epilepsy ( $8.8 \%$ ASD Vs. $1.0 \%$ noASD $)$.

In the adjusted logistic regression analyses, adults with ASD were found to be more likely to have a psychiatric visit $(\mathrm{AOR}=2.63,95 \% \mathrm{CI}=2.41-2.88)$, a non-psychiatric visit $(\mathrm{AOR}=1.07,95 \% \mathrm{CI}=1.01-1.14)$, as well as an injury visit $(\mathrm{AOR}=1.10,95 \% \mathrm{CI}=1.04-1.16)$ as compared to adults without ASD. However, they were significantly less likely to have a psychiatric visit with AUD $(\mathrm{AOR}=0.22,95 \% \mathrm{CI}=0.16-0.29)$ and $\mathrm{SUD}(\mathrm{AOR}=0.19,95 \% \mathrm{CI}=$ $0.14-0.26)$. Adults with ASD were also significantly more likely to have injury visits due to falls $(\mathrm{AOR}=1.48,95 \% \mathrm{CI}=1.34-1.62)$, self-harm $(\mathrm{AOR}=2.95,95 \% \mathrm{CI}=2.33-3.75)$, and 


\section{PhD Dissertation Chapter 3}

suicidal ideation $(\mathrm{AOR}=1.88,95 \% \mathrm{CI}=1.56-2.26)$ as compared to adults without $\mathrm{ASD}$. In addition, adults with ASD were more likely to have multiple cause of injuries as compared to adults without $\mathrm{ASD}(\mathrm{AOR}=1.12,95 \% \mathrm{CI}=1.04-1.19)$. Although non-psychiatric $\mathrm{ED}$ rates for certain visits were lower among adults with ASD, they were seven times more likely to have an ED visit with a principal diagnosis of epilepsy, as compared to adults without ASD (AOR = $7.15,95 \% \mathrm{CI}=6.28-8.13)$.

Mean Total Charges (Table 1 and 6)

Mean total ED charges for adults with ASD were significantly higher as compared to adults without ASD $\left(\right.$ mean $_{\mathrm{ASD}}=\$ 14,289, \mathrm{SE}=\$ 418$ vs. mean $\left._{\mathrm{noASD}}=\$ 6,196, \mathrm{SE}=\$ 165\right)$. Table 6 shows the means and standard errors (SE's) for mean total ED charges among adults with and without ASD by type of ED visits. The mean total charges for a psychiatric visit among adults with ASD were significantly higher as compared to adults without ASD (mean ASD $=\$ 12,506, \mathrm{SE}$ $=\$ 559 \mathrm{vs}$. mean $\left._{\mathrm{noASD}}=\$ 7,238, \mathrm{SE}=\$ 359, \mathrm{p}<0.001\right)$. Despite a lower proportion of adults with ASD having a non-psychiatric visit in the sample, their associated mean total charges were significantly higher $\left(\right.$ mean $_{\mathrm{ASD}}=\$ 13,662, \mathrm{SE}=\$ 681$ vs. mean $\left._{\mathrm{noASD}}=\$ 9,521, \mathrm{SE}=\$ 454, \mathrm{p}<0.001\right)$ when compared to adults without ASD. Injury visits also costed more for adults with ASD as compared to adults without $\mathrm{ASD}\left(\right.$ mean $_{\mathrm{ASD}}=\$ 12,912, \mathrm{SE}=\$ 715$ vs. mean $_{\mathrm{noASD}}=\$ 5,842, \mathrm{SE}=$ $\$ 345, \mathrm{p}<0.001)$.

One of the highest costing ED visit for both adults with (mean $=\$ 75,352, \mathrm{SE}=\$ 10,528)$ and without ASD (mean $=\$ 67,434, \mathrm{SE}=\$ 7,907)$ was an $\mathrm{ED}$ visit with a primary diagnosis of cancer. However, ED visit with a cardiovascular disease had higher mean total ED charges for adults with ASD (mean $=\$ 39,432, \mathrm{SE}=\$ 3,671)$ as compared to adults without $\mathrm{ASD}$ (mean $=$ $\$ 27,181, \mathrm{SE}=\$ 1,893)$. Mean total ED charges for schizophrenia, which was the costliest 


\section{PhD Dissertation Chapter 3}

psychiatric ED visit among adults with $\mathrm{ASD}($ mean $=\$ 20,336, \mathrm{SE}=\$ 1,168)$, were significantly greater than mean charges for adults without $\mathrm{ASD}($ mean $=\$ 12,183, \mathrm{SE}=\$ 1,088)$. Injury visits such as those associated with self-harm also had greater mean total ED charges for adults with $\operatorname{ASD}($ mean $=\$ 13,001, \mathrm{SE}=\$ 1,106)$ as compared to adults without $\mathrm{ASD}($ mean $=\$ 11,560, \mathrm{SE}=$ $\$ 1,810)$, even though the difference did not reach statistical significance. Falls among adults with ASD were also associated with significantly greater mean total ED charges (mean = $\$ 11,230, \mathrm{SE}=\$ 934)$ as compared to falls among adults without $\mathrm{ASD}$ (mean $=\$ 5,880, \mathrm{SE}=$ $\$ 475)$. 


\section{PhD Dissertation Chapter 3}

\section{Discussion}

Our study is a unique contribution to the literature providing information on extent and types of ED use among adults with ASD along with their ED costs. A very recent study showed that an ASD diagnosis was very strongly associated with frequent ED use as well as hospital readmissions (Smith et al., 2015), therefore we expected that the trends of ED visits among adults with ASD will increase. The rates of ED visits with an ASD diagnosis more than doubled in a period of six years in the current study, highlighting a rise in resource utilization among adults with ASD. However, there was a simultaneous decline in mean total ED charges for adults with ASD during this period. The introduction of states mental health parity act was found to be associated with a greater out of pocket spending for individuals with mental health issues such as ASD until 2011 and greater proportion of inpatient admissions spending was shared by the patients during the 2006-2011 period (Carolina-Nicole, John, \& Gregory, 2013). Since the NEDS does not provide information on non-covered charges, the trends in mean total ED charges may not reflect the cost burden of each ED visit to the patient.

In the pooled study sample, ED visits with ASD formed $0.4 \%$ of the total study sample (unmatched). There were two critical findings in the descriptive analyses: 1) Majority (80\%) of adults with ASD were covered by a public health insurance as compared to one-quarter (25\%) adults without ASD. This finding reflects that public payers still account for covering ED and inpatient services among majority of adults with mental health issues such as ASD, consistent with a few previous studies (Ruble et al., 2005; Semansky et al., 2011a); and 2) Another intriguing observation in the study was the difference in rates of inpatient admissions after an ED use among adults with and without ASD. Around one-third of ED visits among adults with ASD led to an inpatient admission as compared to one-tenth of adults without ASD. This indicates that 


\section{PhD Dissertation Chapter 3}

higher ED use among adults with ASD may also lead to greater hospitalizations which is associated with high hospitalization costs (Lokhandwala et al., 2012).

ED visits

The second part of our study focused on identifying the common ED visits among adults with ASD and compare their occurrences to adults without ASD. We found that non-psychiatric $(16 \%)$ and psychiatric (15\%) visits were more common among adults with ASD as compared to adults without ASD. The most commonly associated reasons for ED visits among adults with ASD included: epilepsy (8.8\%), falls (6.5\%), schizophrenia (3.8\%), respiratory disorders $(3.8 \%)$, bipolar disorders (3.2\%), and depression (2.5\%). These rates support the findings regarding comorbid diagnoses among adults with ASD from many other studies (Ahmedani \& Hock, 2012; Leyfer et al., 2006; Maski et al., 2011; Simonoff et al., 2008; White, Oswald, Ollendick, \& Scahill, 2009; Zafeiriou, Ververi, \& Vargiami, 2007).

Psychiatric visit

It is well known that adults with ASD are extensively affected by psychiatric comorbidity, with $90 \%$ reporting at least one DSM-IV psychiatric disorder and our findings indicate that $15 \%$ of adults with ASD are visiting ED due to psychiatric reasons (Leyfer et al., 2006; Lunsky et al., 2009; Palucka \& Lunsky, 2007; Simonoff et al., 2008). Some psychiatric visits were less common among adults with ASD as compared to adults without ASD. These visits were associated with behavioral disorders such as AUD (0.4\% ASD vs. $\left.1.5 \%_{\text {noASD }}\right)$ and SUD ( $0.3 \%$ ASD vs $0.9 \%$ noASD). Literature has shown that usually individuals with ASD are less prone to use of drugs/alcohol, however, individuals on the higher functioning spectrum may frequently engage in alcohol consumption to help alleviate the social difficulties they experience (Santosh \& Mijovic, 2006; Sizoo et al., 2009). Although the prevalence of SUD in our study and in a previous study by Santosh \& Mijovic was low (0.3\%), another study by (Sizoo et al., 2009) has 


\section{PhD Dissertation Chapter 3}

shown a higher prevalence rate $(30 \%)$ of substance abuse among adults with ASD. Since SUD is associated with greater healthcare resource utilization and worse outcomes (Smith et al., 2015), greater attention and monitoring may be needed to identify SUD among adults with ASD so that timely counseling can be provided.

Non-psychiatric visit

Fewer adults with ASD had a non-psychiatric visit with cardiovascular disease (1.4\%), gastrointestinal disease $(0.8 \%)$, and respiratory disorder (3.8\%) as compared to adults with ASD. On the other hand, significantly greater proportion of adults with ASD came to the ED with a principal diagnosis of diabetes $(1.1 \%)$, epilepsy $(8.8 \%)$, and cancer $(0.3 \%)$. Our findings indicate that adults with ASD if not more likely, are at least equally likely to have non-psychiatric needs as compared to adults without ASD when presenting to the ED. Other than epilepsy, most nonpsychiatric disorders among adults with ASD have received little attention, especially in the ED settings. Additional healthcare needs associated with non-psychiatric disorders will only increase the healthcare needs, utilization, and expenditures of adults with ASD.

Injury visit

Adults with ASD had lower rates of injury visits as compared to adults without ASD. Nonetheless, a few specific injury visits were more common in the ASD group. For example, significantly greater proportion of adults with ASD had an injury visit with poisoning (1.4\% ASD vs. $0.8 \%$ noASD), self-harm ( $1.9 \%$ ASD vs. $0.5 \%$ noASD), and suicidal ideation (2.6\% ASD vs. $0.9 \%$ noASD). This finding is complementary to our hypothesis that some of the major reasons for ED use among adults with ASD are disruptive behaviors and self-inflicting injuries. Even after adjusting for other study variables, adults with ASD were more likely to visit ED with a fall injury $(A O R=1.5)$, self-harm $(A O R=2.95)$, and suicidal ideation $(A O R=1.88)$. Most studies have examined injuries, especially falls among patients with developmental disabilities (DD), 


\section{PhD Dissertation Chapter 3}

where the prevalence of injuries have ranged from 11\%-20\% (Finlayson, Morrison, Jackson, Mantry, \& Cooper, 2010; Hsieh, Heller, \& Miller, 2001). The primary risk factors for an injury among adults with DD are higher frequency of seizures, destructive behaviors, and use of antipsychotic drugs. Knowing that adults with ASD may be at a greater risk of all the above factors (Billstedt et al., 2007; Esbensen et al., 2009; Levy et al., 2010), their probability of a fall injury and a subsequent ED visit is also very high.

In the current study, although the rates of injuries due to assault were lower among adults with ASD as compared to adults without ASD, further investigation is needed to corroborate this finding using other data (for e.g., police records). This is a critical type of injury especially for adults with ASD who many a time have difficulty communicating their needs and experiences resulting in frustration, anger, aggression, and self-injurious behaviors. Future studies should focus on examining the gravity of such communication impediments on both self-inflictions as well as receipt of aggression by others during ED visits and hospitalizations.

\section{Total ED Charges}

Our study also compared mean total ED charges for adults with and without ASD. Adults with ASD, in general had higher ED costs compared to adults without ASD (ratio of means ASD vs. NoASD $=2.30, \mathrm{p}<0.001)$. The ratio of means ASD vs. NoASD for specific ED visits (psychiatric disorders $=1.7$; non-psychiatric disorders $=1.4$; and injuries $=2.2$ ) indicated that the biggest difference in mean total ED charges between ASD and no ASD group was attributed to injuries. Mean charges of injuries due to falls among adults with ASD was almost double the costs for falls among adults without ASD ( $\mathrm{p}<0.001)$. Mean charges of ED visit with suicidal ideation, which is a common diagnosis among adults with ASD, was also significantly higher among the ASD group as compared to the no ASD group (ratio of means $\mathrm{ASD}_{\text {vs. }}$ NoASD $=1.56, \mathrm{p}<0.001$ ). 


\section{PhD Dissertation Chapter 3}

These findings indicate that injuries are common among adults with ASD (Kato et al., 2013) and are associated with high ED utilization and costs. Since, one-third of adults with ASD in the sample had an inpatient admission after using ED, the higher mean total ED charges not only reflect outpatient ED costs, but also indicate costs contributed by hospitalizations.

\section{Implications}

The current study has implications for policy discussions related to quality of care and care coordination in a primary care/specialty care setting for adults with ASD. Our study sheds light on the need for better guidelines and greater support for incorporating ASD related training of physicians and other healthcare providers who usually report lack of self-perceived competency in treating and diagnosing adults with ASD (Bruder et al., 2012; Golnik et al., 2009; Oskoui \& Wolfson, 2012). (Miller, 2015) examined the extent of ASD knowledge among nurses working in an ED and found that more than half of ED nurses surveyed reported having accurate knowledge, correctly identified causes of visit and comorbidities, and chose appropriate interventions for ASD cases. However, nurses still reported having limited knowledge and resources available to them and felt a need for ASD education early on in their training. This perceived need to overcome gaps in ASD related care among nurses may also be true for physicians and other healthcare providers. Future studies should examine the association of quality and consistency of care received by adults with ASD in the primary setting with a patient's subsequent ED use, frequency of ED use, hospital admissions, and healthcare costs. 


\section{PhD Dissertation Chapter 3}

\section{Limitations}

Despite many advantages, the NEDS data is limited by use of a discharge level rather than a person level data. Therefore, multiple visits by a much sicker population could not be distinguished. Similar to Kalb et al. (2012) who utilized the NEDS for examining ED visits among children with ASD, we defined ASD as any of the 15 possible diagnoses rather than a primary diagnosis. However, this algorithm has not been validated in survey research and may have its own drawbacks. In this study we also assumed that ED visits with a primary psychiatric disorder and non-psychiatric disorder was actually correlated with a patient's psychiatric and non-psychiatric needs at the time of the visit. It is quite possible that the reason(s) for an ED visit might have been completely different than the principal diagnosis on the record, which could have been used purely for billing purposes.

The data allowed only for capturing the total ED charges for the services used/billed. We could not manipulate the data to deliver cost/expenditure, which is a better and a more meaningful concept. However, the requirement of the study was to identify the excess healthcare utilization and total charges for the ASD group as compared to the no ASD group and using charges sufficed the need of the current study goals. We also could not account for the charges that were not included in the ED and inpatient charges (such as professional fees), expenditures paid by the payer, and/or out of pocket expenditures for the patients, which would provide the cost sharing burden among patients with or without ASD. The data also was limited by the absence of patient reported health behaviors (such as smoking, tobacco use, and drug abuse), physician review charts, and other additional information that could help validate the reasons for ED visits. Finally, racial/ethnic differences could not be accounted for in the study due to the lack of information on race in the available data. 


\section{PhD Dissertation Chapter 3}

\section{Conclusion}

Rates of ED visits among adults with ASD are on the rise. Adults with ASD use ED for different reasons which are not restricted to psychiatric needs, but also extend to non-psychiatric needs and injuries. ED visits among adults with ASD are also associated with significantly greater hospitalization rates as compared to adults without ASD. Mean total ED charges for adults with ASD are almost twice as high as charges for adults without ASD. Prevalence, risk factors, and burden of ED utilization among adults with ASD is understudied and future studies should examine the impact of such ED utilization on long term healthcare costs. 


\section{PhD Dissertation Chapter 3}

\section{References}

Ahmedani, B. K., \& Hock, R. M. (2012). Health care access and treatment for children with co-morbid autism and psychiatric conditions. Social Psychiatry and Psychiatric Epidemiology, 47(11), 1807-1814. doi:10.1007/s00127012-0482-0; 10.1007/s00127-012-0482-0

Billstedt, E., Gillberg, I. C., \& Gillberg, C. (2005). Autism after adolescence: Population-based 13- to 22-year follow-up study of 120 individuals with autism diagnosed in childhood. Journal of Autism and Developmental Disorders, 35(3), 351-360.

Billstedt, E., Gillberg, I. C., \& Gillberg, C. (2007). Autism in adults: Symptom patterns and early childhood predictors. Use of the DISCO in a community sample followed from childhood. Journal of Child Psychology and Psychiatry, and Allied Disciplines, 48(11), 1102-1110. doi:JCPP1774 [pii]

Bruder, M. B., Kerins, G., Mazzarella, C., Sims, J., \& Stein, N. (2012). Brief report: The medical care of adults with autism spectrum disorders: Identifying the needs. Journal of Autism and Developmental Disorders, 42(11), 24982504. doi:10.1007/s10803-012-1496-x [doi]

Brugha, T. S., McManus, S., Bankart, J., Scott, F., Purdon, S., Smith, J., . . Meltzer, H. (2011). Epidemiology of autism spectrum disorders in adults in the community in england. Archives of General Psychiatry, 68(5), 459-465. doi:10.1001/archgenpsychiatry.2011.38; 10.1001/archgenpsychiatry.2011.38

Bureau of Labor Statistics, US Department of Labor. (2014). Archived consumer price index detailed report information. Retrieved from http://www.bls.gov/cpi/cpi_dr.htm

Carolina-Nicole, H., John, H., \& Gregory, S. (2013). The impact of the mental health parity and addiction equity act on inpatient admissions . (Issue Brief No. 5). Washington D.C.: Healthcare Cost Institute.

Choudhry, L., Dougless, M., Lewis, J., Olson, C. H., Osterman, R., \& Shah, P. (2007). The impact of community health centers \& community-affiliated health plans on emergency department use. ().National Association of Community Health Centers; Association for Community Affiliated Plans.

Croen, L. A., Najjar, D. V., Ray, G. T., Lotspeich, L., \& Bernal, P. (2006). A comparison of health care utilization and costs of children with and without autism spectrum disorders in a large group-model health plan. Pediatrics, 118(4), e1203-11. doi:118/4/e1203 [pii]

Croen, L. A., Zerbo, O., Qian, Y., Massolo, M. L., Rich, S., Sidney, S., \& Kripke, C. (2015). The health status of adults on the autism spectrum. Autism: The International Journal of Research and Practice, doi:1362361315577517 [pii]

Curran, G. M., Sullivan, G., Williams, K., Han, X., Collins, K., Keys, J., \& Kotrla, K. J. (2003). Emergency department use of persons with comorbid psychiatric and substance abuse disorders. Annals of Emergency Medicine, 41(5), 659-667. doi:10.1067/mem.2003.154 [doi]

Doshi, A., Boudreaux, E. D., Wang, N., Pelletier, A. J., \& Camargo, C. A.,Jr. (2005). National study of US emergency department visits for attempted suicide and self-inflicted injury, 1997-2001. Annals of Emergency Medicine, 46(4), 369-375. doi:S0196-0644(05)00525-1 [pii]

Esbensen, A. J., Greenberg, J. S., Seltzer, M. M., \& Aman, M. G. (2009). A longitudinal investigation of psychotropic and non-psychotropic medication use among adolescents and adults with autism spectrum disorders. Journal of Autism and Developmental Disorders, 39(9), 1339-1349. doi:10.1007/s10803-009-0750-3 [doi]

Finlayson, J., Morrison, J., Jackson, A., Mantry, D., \& Cooper, S. A. (2010). Injuries, falls and accidents among adults with intellectual disabilities. Prospective cohort study. Journal of Intellectual Disability Research: JIDR, 54(11), 966-980. doi:10.1111/j.1365-2788.2010.01319.x [doi] 


\section{PhD Dissertation Chapter 3}

Golnik, A., Ireland, M., \& Borowsky, I. W. (2009). Medical homes for children with autism: A physician survey. Pediatrics, 123(3), 966-971. doi:10.1542/peds.2008-1321 [doi]

Healthcare Cost and Utilization Project (HCUP). (2011). HCUP nationwide emergency department sample (NEDS). (). Rockville, MD: Agency for Healthcare Research and Quality.

Hofvander, B., Delorme, R., Chaste, P., Nyden, A., Wentz, E., Stahlberg, O., . . Leboyer, M. (2009). Psychiatric and psychosocial problems in adults with normal-intelligence autism spectrum disorders. BMC Psychiatry, 9, 35244X-9-35. doi:10.1186/1471-244X-9-35; 10.1186/1471-244X-9-35

Hsieh, K., Heller, T., \& Miller, A. B. (2001). Risk factors for injuries and falls among adults with developmental disabilities. Journal of Intellectual Disability Research: JIDR, 45(Pt 1), 76-82. doi:jir277 [pii]

Kalb, L. G., Stuart, E. A., Freedman, B., Zablotsky, B., \& Vasa, R. (2012). Psychiatric-related emergency department visits among children with an autism spectrum disorder. Pediatric Emergency Care, 28(12), 1269-1276. doi:10.1097/PEC.0b013e3182767d96 [doi]

Kato, K., Mikami, K., Akama, F., Yamada, K., Maehara, M., Kimoto, K., . . Matsumoto, H. (2013). Clinical features of suicide attempts in adults with autism spectrum disorders. General Hospital Psychiatry, 35(1), 50-53. doi:10.1016/j.genhosppsych.2012.09.006 [doi]

Levy, S. E., Giarelli, E., Lee, L. C., Schieve, L. A., Kirby, R. S., Cunniff, C., . . Rice, C. E. (2010). Autism spectrum disorder and co-occurring developmental, psychiatric, and medical conditions among children in multiple populations of the United States. Journal of Developmental and Behavioral Pediatrics: JDBP, 31(4), 267-275. doi:10.1097/DBP.0b013e3181d5d03b; 10.1097/DBP.0b013e3181d5d03b

Leyfer, O. T., Folstein, S. E., Bacalman, S., Davis, N. O., Dinh, E., Morgan, J., . . Lainhart, J. E. (2006). Comorbid psychiatric disorders in children with autism: Interview development and rates of disorders. Journal of Autism and Developmental Disorders, 36(7), 849-861. doi:10.1007/s10803-006-0123-0 [doi]

Lokhandwala, T., Khanna, R., \& West-Strum, D. (2012). Hospitalization burden among individuals with autism. Journal of Autism and Developmental Disorders, 42(1), 95-104. doi:10.1007/s10803-011-1217-x [doi]

Lugnegard, T., Hallerback, M. U., \& Gillberg, C. (2011a). Psychiatric comorbidity in young adults with a clinical diagnosis of asperger syndrome. Research in Developmental Disabilities, 32(5), 1910-1917. doi:10.1016/j.ridd.2011.03.025; 10.1016/j.ridd.2011.03.025

Lugnegard, T., Hallerback, M. U., \& Gillberg, C. (2011b). Psychiatric comorbidity in young adults with a clinical diagnosis of asperger syndrome. Research in Developmental Disabilities, 32(5), 1910-1917.

doi:10.1016/j.ridd.2011.03.025 [doi]

Lunsky, Y., Gracey, C., \& Bradley, E. (2009). Adults with autism spectrum disorders using psychiatric hospitals in ontario: Clinical profile and service needs. Research in Autism Spectrum Disorders, 3(4), 1006-1013.

Magiati, I., Tay, X. W., \& Howlin, P. (2014). Cognitive, language, social and behavioral outcomes in adults with autism spectrum disorders: A systematic review of longitudinal follow-up studies in adulthood. Clinical Psychology Review, 34(1), 73-86. doi:10.1016/j.cpr.2013.11.002 [doi]

Maski, K. P., Jeste, S. S., \& Spence, S. J. (2011). Common neurological co-morbidities in autism spectrum disorders. Current Opinion in Pediatrics, 23(6), 609-615. doi:10.1097/MOP.0b013e32834c9282; 10.1097/MOP.0b013e32834c9282

Matson, J. L., \& Rivet, T. T. (2008). Characteristics of challenging behaviors in adults with autistic disorder, PDDNOS, and intellectual disability. Journal of Intellectual \& Developmental Disability, 33(4), 323-329.

doi:10.1080/13668250802492600; 10.1080/13668250802492600 


\section{PhD Dissertation Chapter 3}

Miller, S. M. (2015). Nurses caring for adults with autism in an emergency department: A survey of knowledge. Doctoral Projects, (7)

Nicolaidis, C., Raymaker, D., McDonald, K., Dern, S., Boisclair, W. C., Ashkenazy, E., \& Baggs, A. (2013). Comparison of healthcare experiences in autistic and non-autistic adults: A cross-sectional online survey facilitated by an academic-community partnership. Journal of General Internal Medicine, 28(6), 761-769. doi:10.1007/s11606012-2262-7 [doi]

Oskoui, M., \& Wolfson, C. (2012). Treatment comfort of adult neurologists in childhood onset conditions. The Canadian Journal of Neurological Sciences.Le Journal Canadien Des Sciences Neurologiques, 39(2), 202-205. doi:HM223822024126U6 [pii]

Owens, P. L., Barrett, M. L., Gibson, T. B., Andrews, R. M., Weinick, R. M., \& Mutter, R. L. (2010). Emergency department care in the United States: A profile of national data sources. Annals of Emergency Medicine, 56(2), 150165. doi:10.1016/j.annemergmed.2009.11.022 [doi]

Palucka, A. M., \& Lunsky, Y. (2007). Review of inpatient admissions of individuals with autism spectrum disorders to a specialized dual diagnosis program. Journal on Developmental Disabilities, 13(1), 205-209.

Pines, J. M., Asplin, B. R., Kaji, A. H., Lowe, R. A., Magid, D. J., Raven, M., . . Yealy, D. M. (2011). Frequent users of emergency department services: Gaps in knowledge and a proposed research agenda. Academic Emergency Medicine: Official Journal of the Society for Academic Emergency Medicine, 18(6), e64-9. doi:10.1111/j.15532712.2011.01086.x [doi]

Richmond, T. S., Hollander, J. E., Ackerson, T. H., Robinson, K., Gracias, V., Shults, J., \& Amsterdam, J. (2007). Psychiatric disorders in patients presenting to the emergency department for minor injury. Nursing Research, 56(4), 275-282. doi:10.1097/01.NNR.0000280616.13566.84 [doi]

Ruble, L. A., Heflinger, C. A., Renfrew, J. W., \& Saunders, R. C. (2005). Access and service use by children with autism spectrum disorders in medicaid managed care. Journal of Autism and Developmental Disorders, 35(1), 3-13.

Santosh, P. J., \& Mijovic, A. (2006). Does pervasive developmental disorder protect children and adolescents against drug and alcohol use? European Child \& Adolescent Psychiatry, 15(4), 183-188. doi:10.1007/s00787-0050517-0 [doi]

Semansky, R. M., Xie, M., \& Mandell, D. S. (2011). Medicaid's increasing role in treating youths with autism spectrum disorders. Psychiatric Services (Washington, D.C.), 62(6), 588. doi:10.1176/appi.ps.62.6.588; 10.1176/appi.ps.62.6.588

Sills, M. R., \& Bland, S. D. (2002). Summary statistics for pediatric psychiatric visits to US emergency departments, 1993-1999. Pediatrics, 110(4), e40.

Simonoff, E., Pickles, A., Charman, T., Chandler, S., Loucas, T., \& Baird, G. (2008). Psychiatric disorders in children with autism spectrum disorders: Prevalence, comorbidity, and associated factors in a population-derived sample. Journal of the American Academy of Child and Adolescent Psychiatry, 47(8), 921-929.

doi:10.1097/CHI.0b013e318179964f [doi]

Sizoo, B., van den Brink, W., Gorissen van Eenige, M., \& van der Gaag, R. J. (2009). Personality characteristics of adults with autism spectrum disorders or attention deficit hyperactivity disorder with and without substance use disorders. The Journal of Nervous and Mental Disease, 197(6), 450-454. doi:10.1097/NMD.0b013e3181a61dd0 [doi]

Smith, M. W., Stocks, C., \& Santora, P. B. (2015). Hospital readmission rates and emergency department visits for mental health and substance abuse conditions. Community Mental Health Journal, 51(2), 190-197.

doi:10.1007/s10597-014-9784-x [doi] 


\section{PhD Dissertation Chapter 3}

Soto, E. C., Frederickson, A. M., Trivedi, H., Le, A., Eugene, M. C., Shekher, M., . . Correll, C. U. (2009). Frequency and correlates of inappropriate pediatric psychiatric emergency room visits. The Journal of Clinical Psychiatry, 70(8), 1164-1177. doi:10.4088/JCP.08m04839 [doi]

Tang, N., Stein, J., Hsia, R. Y., Maselli, J. H., \& Gonzales, R. (2010). Trends and characteristics of US emergency department visits, 1997-2007. JAMA: The Journal of the American Medical Association, 304(6), 664-670. doi:10.1001/jama.2010.1112 [doi]

Wharff, E. A., Ginnis, K. B., Ross, A. M., \& Blood, E. A. (2011). Predictors of psychiatric boarding in the pediatric emergency department: Implications for emergency care. Pediatric Emergency Care, 27(6), 483-489. doi:10.1097/PEC.0b013e31821d8571 [doi]

White, S. W., Oswald, D., Ollendick, T., \& Scahill, L. (2009). Anxiety in children and adolescents with autism spectrum disorders. Clinical Psychology Review, 29(3), 216-229. doi:10.1016/j.cpr.2009.01.003;

10.1016/j.cpr.2009.01.003

Williams, E. R., Guthrie, E., Mackway-Jones, K., James, M., Tomenson, B., Eastham, J., \& McNally, D. (2001). Psychiatric status, somatisation, and health care utilization of frequent attenders at the emergency department: A comparison with routine attenders. Journal of Psychosomatic Research, 50(3), 161-167. doi:S0022-3999(00)002282 [pii]

Zafeiriou, D. I., Ververi, A., \& Vargiami, E. (2007). Childhood autism and associated comorbidities. Brain \& Development, 29(5), 257-272. doi:S0387-7604(06)00209-9 [pii] 
Figures

\section{ED Rates for Adults with ASD Nationwide Emergency Database Sample 2006-2011, Healthcare Cost and Utilization Project, AHRQ}

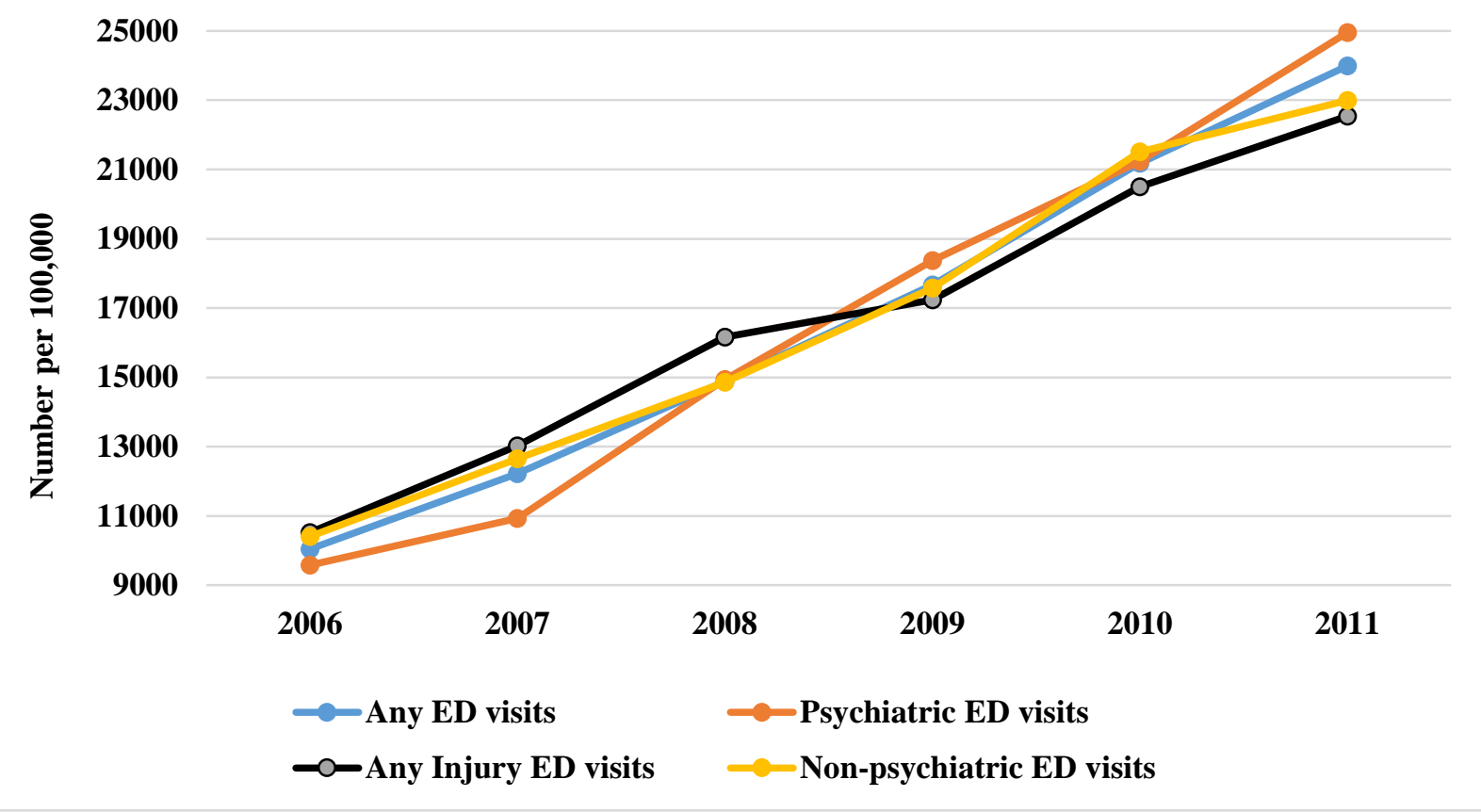

Figure 1. Number of ED visits with ASD per every 100,000 ED admissions for all, psychiatric, nonpsychiatric, and any injury ED visits.

Trend analyses examined change in rates of ED visits associated with ASD as compared to ED visits without ASD from 2006 to 2011. In the logistic regression analyses, the trends were adjusted for hospital region, urban/rural status, median annual household income, and primary payer.

All trends for ASD were insignificant at $\mathrm{p} \leq 0.05$ level 


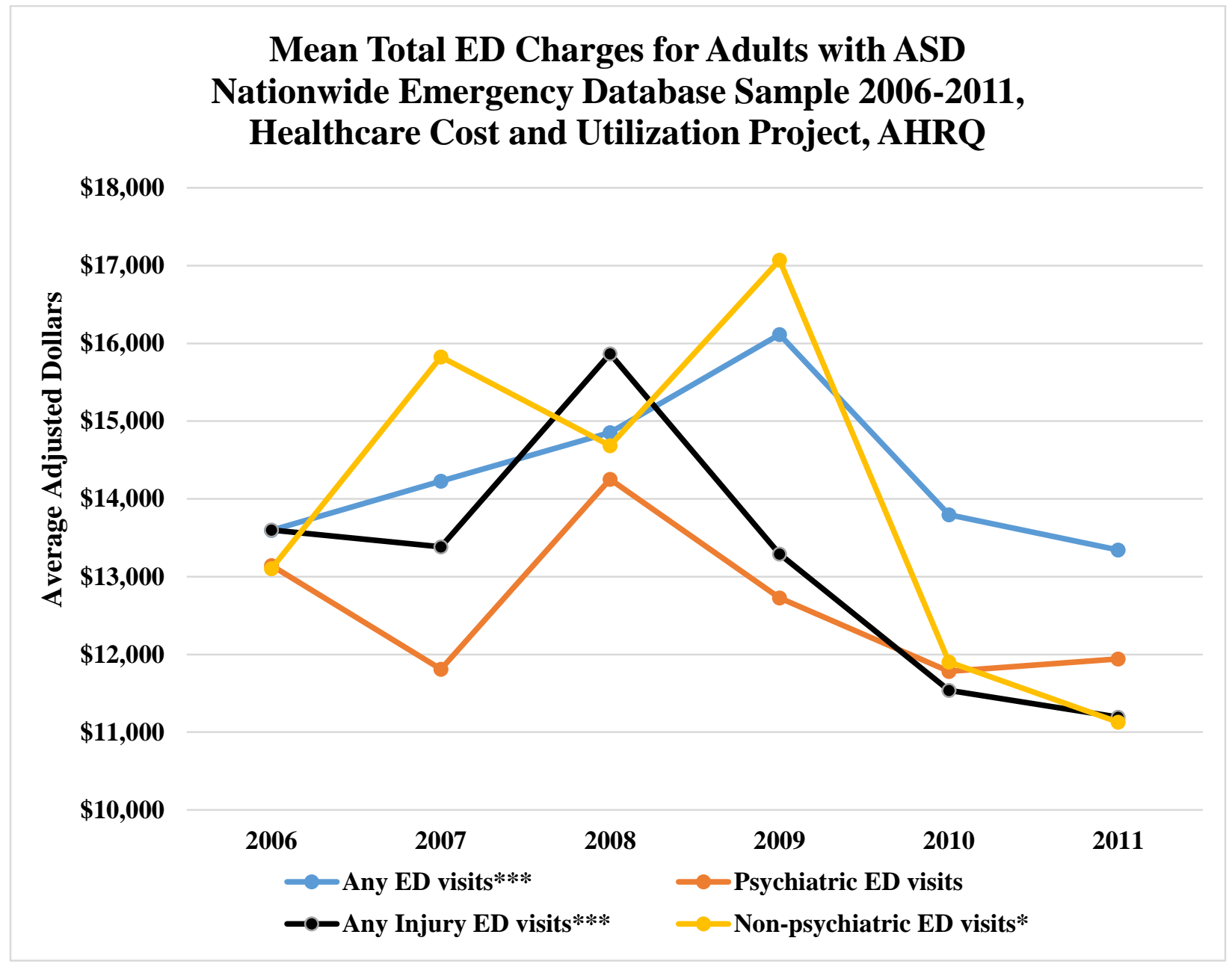

Figure 2. Mean inflation adjusted total ED charges for ASD visits for all, psychiatric, non-psychiatric, and any injury ED visits.

Trend analyses examined the change in inflation adjusted mean total ED charges for ED visits associated with ASD as compared to ED visits without ASD from 2006 to 2011.In the linear regression analyses, the trends were adjusted for age, gender, hospital region, urban/rural status, median annual household income, and primary payer.

Trend for mean total charges for psychiatric ED visits with ASD was insignificant at $\mathrm{p} \leq 0.05$ level. Sig.: ***P $<0.001 ; * * 0.001 \leq \mathrm{P}<0.01 ; * 0.01 \leq \mathrm{P}<0.05$. 
PhD Dissertation Chapter 3

Tables

\begin{tabular}{|c|c|c|c|c|c|}
\hline $\begin{array}{l}\text { Description of Poole } \\
\text { Adults with and } \\
\text { 2006-2011 Nationwic }\end{array}$ & $\begin{array}{l}\text { le } 1 \\
\text { ple Chara } \\
\text { matchec } \\
\text { Departm }\end{array}$ & $\begin{array}{l}\text { tics by } \\
\text { ge and } \\
\text { ample }\end{array}$ & $\begin{array}{l}\text { D status } \\
\text { nder } \\
=102,10\end{array}$ & & \\
\hline & & & No & & Sig \\
\hline & & Wt. & & Wt. & \\
\hline & $\mathrm{N}$ & $\%$ & $\mathrm{~N}$ & $\%$ & \\
\hline Sex & & & & & \\
\hline Male & 19,286 & 75.5 & 57,858 & 75.7 & ns \\
\hline Female & 6,241 & 24.5 & 18,723 & 24.3 & \\
\hline Age (in years) & & & & & \\
\hline $22-40$ & 17,961 & 70.2 & 53,883 & 70.3 & ns \\
\hline $41-54$ & 5,575 & 21.8 & 16,725 & 21.9 & \\
\hline $55-64$ & 1,991 & 8.0 & 5,973 & 7.8 & \\
\hline Income Groups (quartiles) & & & & & $* * *$ \\
\hline Q1 & 5,364 & 21.2 & 24,787 & 32.4 & \\
\hline Q2 & 6,230 & 24.5 & 20,783 & 27.2 & \\
\hline Q3 & 6,590 & 25.8 & 16,848 & 21.9 & \\
\hline Q4 & 6,612 & 25.8 & 12,119 & 15.8 & \\
\hline Missing & 731 & 2.8 & 2,044 & 2.7 & \\
\hline Primary Payer & & & & & $* * *$ \\
\hline Public & 20,483 & 80.4 & 19,496 & 25.7 & \\
\hline Private & 3,764 & 14.7 & 25,938 & 34.3 & \\
\hline Self-Charge & 807 & 3.2 & 23,934 & 31.1 & \\
\hline No Charge/Other & 424 & 1.7 & 6,763 & 9.0 & \\
\hline Hospital Region & & & & & $* * *$ \\
\hline North-east & 7,453 & 29.6 & 15,211 & 20.5 & \\
\hline Mid-west & 6,446 & 27.0 & 16,366 & 23.5 & \\
\hline South & 7,476 & 27.1 & 32,132 & 38.8 & \\
\hline West & 4,152 & 16.3 & 12,872 & 17.2 & \\
\hline Hospital Location & & & & & \\
\hline Rural & 945 & 4.0 & 5,345 & 7.3 & $* * *$ \\
\hline Urban & 24,459 & 95.5 & 70,503 & 91.8 & \\
\hline Missing & 123 & 0.5 & 733 & 1.0 & \\
\hline ED Event & & & & & $* * *$ \\
\hline Treat and release & 16,340 & 64.0 & 66,751 & 87.1 & \\
\hline Inpatient admission & 8,651 & 33.9 & 8,136 & 10.6 & \\
\hline Transfer & 326 & 1.3 & 804 & 1.1 & \\
\hline Died/other & 210 & 0.8 & 890 & 1.2 & \\
\hline Mortality Events & 181 & 0.7 & 16,186 & 0.3 & $* * *$ \\
\hline Total ED charges mean (SE) & $\$ 14$ & $\$ 418)$ & & $\$ 165)$ & $* * *$ \\
\hline
\end{tabular}


Table 2

Type of ED Visits in the Pooled Study Sample by ASD Status

Adults with and without ASD matched on age and gender

2006-2011 Nationwide Emergency Department Sample ( $\mathrm{n}=102,108)$

\begin{tabular}{|c|c|c|c|c|c|c|}
\hline & & \multicolumn{2}{|c|}{ ASD } & \multicolumn{2}{|c|}{ No ASD } & \multirow[t]{2}{*}{ Sig } \\
\hline & & $\mathrm{N}$ & Col. Wt.\% & $\mathrm{N}$ & Col. Wt.\% & \\
\hline \multirow[t]{11}{*}{ Psychiatric Visit } & & 3,831 & 15.1 & 3,174 & 4.2 & $* * *$ \\
\hline & ADD/ADHD & 328 & 1.3 & 27 & 0.0 & **** \\
\hline & Adjustment Disorders & 137 & 0.5 & 91 & 0.1 & $* * *$ \\
\hline & Anxiety Disorders & 376 & 1.5 & 675 & 0.9 & $* * *$ \\
\hline & AUD & 97 & 0.4 & 1,099 & 1.5 & $* * *$ \\
\hline & Bipolar Disorder & 825 & 3.2 & 326 & 0.4 & $* * *$ \\
\hline & Depression & 608 & 2.5 & 717 & 1.0 & $* * *$ \\
\hline & Intellectual Disabilities & 399 & 1.6 & 22 & 0.0 & $* * *$ \\
\hline & Personality Disorders & 113 & 0.4 & 11 & 0.0 & $* * *$ \\
\hline & Schizophrenia & 971 & 3.8 & 619 & 0.8 & $* * *$ \\
\hline & SUD & 74 & 0.3 & 686 & 0.9 & $* * *$ \\
\hline \multirow[t]{13}{*}{ Injury Visit ${ }^{\S \S}$} & & 6,076 & 23.7 & 21,117 & 27.7 & $* * *$ \\
\hline & Assault $^{€ \S}$ & 146 & 0.9 & 901 & 1.9 & *** \\
\hline & Cut & 346 & 1.3 & 2,270 & 3.0 & $* * *$ \\
\hline & Drown & 5 & 0.0 & 6 & 0.0 & $\mathrm{nc}$ \\
\hline & Fall & 1,655 & 6.5 & 3,751 & 5.0 & $* * *$ \\
\hline & Fire & 52 & 0.2 & 323 & 0.4 & $* * *$ \\
\hline & Machinery & 1 & 0.0 & 177 & 0.2 & $\mathrm{nc}$ \\
\hline & Poison & 368 & 1.4 & 567 & 0.8 & $* * *$ \\
\hline & Self-harm ${ }^{\S \S}$ & 475 & 1.9 & 371 & 0.5 & $* * *$ \\
\hline & Struck & 832 & 3.3 & 3,337 & 4.4 & $* * *$ \\
\hline & Suffocation & 66 & 0.3 & 24 & 0.0 & $* * *$ \\
\hline & Suicidal Ideation ${ }^{\S \S}$ & 650 & 2.6 & 683 & 0.9 & $* * *$ \\
\hline & Multiple Injuries $^{\S \S}$ & 3,754 & 14.7 & 12,180 & 16.3 & **** \\
\hline \multirow[t]{7}{*}{ Non-psychiatric Visit } & & 4,130 & 16.1 & 10,444 & 13.6 & $* * *$ \\
\hline & Cancer & 70 & 0.3 & 147 & 0.2 & *** \\
\hline & Cardiovascular Disease & 369 & 1.4 & 1,867 & 2.5 & $* * *$ \\
\hline & Diabetes & 274 & 1.1 & 628 & 0.8 & $* *$ \\
\hline & Epilepsy & 2,253 & 8.8 & 793 & 1.0 & $* *$ \\
\hline & Gastrointestinal Disease & 188 & 0.8 & 765 & 1.0 & $* *$ \\
\hline & Respiratory Disease & 976 & 3.8 & 6,244 & 8.1 & $* *$ \\
\hline
\end{tabular}

The estimates are provided from NEDS representing ED visits with adults aged 22-64 years from the years 20062011; ED visits with and without ASD were matched on age and gender; nc: Not conclusive. Tests not feasible due to low cell sizes;

ADD/ADHD: Attention deficit disorders/attention deficit hyperactivity disorders; AUD: Alcohol use disorders; SUD: Substance use disorders.

Col. Wt. \%: Column weighted percentages. Represents percentages within ASD and no ASD ED visits that were associated with each individual diagnoses. The denominator is the total number of ED visits with an ASD diagnosis $(\mathrm{n}=25,527)$ and without an ASD diagnosis $(\mathrm{n}=76,581)$.

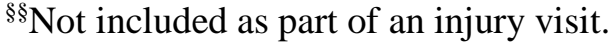

${ }^{\epsilon}$ Data on assault intent was only available for the years 2009-2011.

Sig.: ***P $<0.001 ; * * 0.001 \leq \mathrm{P}<0.01 ; * 0.01 \leq \mathrm{P}<0.05$. 
PhD Dissertation Chapter 3

Table 3

Odds Ratios and Confidence Intervals from Multivariate Logistic Regressions for Psychiatric Visits Adults with and without ASD matched on age and gender 2006-2011 Nationwide Emergency Department Sample $(\mathrm{n}=102,108)$

\begin{tabular}{lcccccc}
\hline & OR & $95 \%$ CI & Sig. & AOR & $95 \%$ CI & Sig. \\
\hline Any & 4.03 & $(3.74,4.34)$ & $* * *$ & 2.63 & $(2.41,2.88)$ & $* * *$ \\
Schizophrenia & 4.77 & $(4.17,5.46)$ & $* * *$ & 1.90 & $(1.61,2.23)$ & $* * *$ \\
ADD/ADHD & 38.64 & $(25.96,57.51)$ & $* * *$ & 33.82 & $(22.01,51.97)$ & $* * *$ \\
Adjustment Disorders & 4.33 & $(3.25,5.77)$ & $* * *$ & 4.04 & $(2.62,6.23)$ & $* * *$ \\
Anxiety & 1.70 & $(1.46,1.99)$ & $* * *$ & 1.93 & $(1.58,2.36)$ & $* * *$ \\
AUD & 0.26 & $(0.20,0.33)$ & $* * *$ & 0.22 & $(0.16,0.29)$ & $* * *$ \\
Bipolar Disorders & 7.84 & $(6.71,9.16)$ & $* * *$ & 3.83 & $(3.15,4.66)$ & $* * *$ \\
Depression & 2.57 & $(2.24,2.96)$ & $* * *$ & 1.86 & $(1.54,2.24)$ & $* * *$ \\
Intellectual Disabilities & 54.12 & $(35.24,83.12)$ & $* * *$ & 41.31 & $(24.56,69.48)$ & $* * *$ \\
Personality Disorders & 30.55 & $(16.02,58.24)$ & $* * *$ & 24.57 & $(11.22,53.80)$ & $* * *$ \\
SUD & 0.30 & $(0.24,0.40)$ & $* * *$ & 0.19 & $(0.14,0.26)$ & $* * *$ \\
\hline
\end{tabular}

The estimates are provided from NEDS representing ED visits with adults aged 22-64 years from the years 2006-2011; ED visits with and without ASD were matched on age and gender;

ADD/ADHD: Attention deficit disorders/attention deficit hyperactivity disorders; AUD: Alcohol use disorders; SUD: Substance use disorders.

OR: Odds ratios; AOR: Adjusted odds ratios; CI: Confidence Intervals.

Multivariate logistic regressions were adjusted for sex, age, NEDS year, income groups, primary payer, hospital region, hospital location, and ED event.

Sig.: *** $\mathrm{P}<0.001 ; * * 0.001 \leq \mathrm{P}<0.01 ; * 0.01 \leq \mathrm{P}<0.05$.

Table 4

Odds Ratios and Confidence Intervals from Multivariate Logistic Regressions for Non-Psychiatric Visits Adults with and without ASD matched on age and gender 2006-2011 Nationwide Emergency Department Sample $(\mathrm{n}=102,108)$

\begin{tabular}{lcccccc}
\hline & OR & $95 \%$ CI & Sig. & AOR & $95 \%$ CI & Sig. \\
\hline Any & 1.22 & $(1.16,1.29)$ & $* * *$ & 1.07 & $(1.01,1.14)$ & $*$ \\
Cancer & 1.50 & $(1.11,2.02)$ & $* *$ & 0.65 & $(0.46,0.91)$ & $*$ \\
Cardiovascular Disease & 0.58 & $(0.51,0.66)$ & $* * *$ & 0.39 & $(0.33,0.45)$ & $* * *$ \\
Diabetes & 1.32 & $(1.10,1.57)$ & $* *$ & 0.75 & $(0.61,0.93)$ & $* *$ \\
Epilepsy & 9.26 & $(8.36,10.27)$ & $* * *$ & 7.15 & $(6.28,8.13)$ & $* * *$ \\
Gastrointestinal Disease & 0.76 & $(0.64,0.91)$ & $* *$ & 0.66 & $(0.54,0.82)$ & $* * *$ \\
Respiratory Disease & 0.45 & $(0.41,0.49)$ & $* * *$ & 0.53 & $(0.48,0.58)$ & $* * *$ \\
\hline
\end{tabular}

The estimates are provided from NEDS representing ED visits with adults aged 22-64 years from the years 2006-2011; ED visits with and without ASD were matched on age and gender;

OR: Odds ratios; AOR: Adjusted odds ratios; CI: Confidence Intervals.

Multivariate logistic regressions were adjusted for sex, age, NEDS year, income groups, primary payer, hospital region, hospital location, and ED event.

Sig.: $* * * \mathrm{P}<0.001 ; * * 0.001 \leq \mathrm{P}<0.01 ; * 0.01 \leq \mathrm{P}<0.05$ 
PhD Dissertation Chapter 3

Table 5

Odds Ratios and Confidence Intervals from Multivariate Logistic Regressions for Injury Visits

Adults with and without ASD matched on age and gender

2006-2011 Nationwide Emergency Department Sample $(\mathrm{n}=102,108)$

\begin{tabular}{lcccccc}
\hline & OR & $95 \%$ CI & Sig. & AOR & $95 \%$ CI & Sig. \\
\hline Any ${ }^{\S \S}$ & 0.81 & $(0.78,0.85)$ & $* * *$ & 1.10 & $(1.04,1.16)$ & $* * *$ \\
Assault & 0.47 & $(0.38,0.57)$ & $* * *$ & 0.61 & $(0.49,0.77)$ & $* * *$ \\
Cut & 0.44 & $(0.38,0.50)$ & $* * *$ & 0.75 & $(0.65,0.86)$ & $* * *$ \\
Fall & 1.33 & $(1.23,1.44)$ & $* * *$ & 1.48 & $(1.34,1.62)$ & $* * *$ \\
Poison & 1.91 & $(1.66,2.20)$ & $* * *$ & 1.21 & $(1.00,1.46)$ & $\mathrm{ns}$ \\
Self-harm & 3.90 & $(3.30,4.60)$ & $* * *$ & 2.95 & $(2.33,3.75)$ & $* * *$ \\
Struck & 0.74 & $(0.67,0.82)$ & $* * *$ & 1.10 & $(0.99,1.22)$ & $\mathrm{ns}$ \\
Suicidal Ideation & 2.96 & $(2.59,3.40)$ & $* * *$ & 1.88 & $(1.56,2.26)$ & $* * *$ \\
Multiple Injuries & 0.89 & $(0.84,0.95)$ & $* * *$ & 1.12 & $(1.04,1.19)$ & $* *$ \\
\hline
\end{tabular}

The estimates are provided from NEDS representing ED visits with adults aged 22-64 years from the years 2006-2011; ED visits with and without ASD were matched on age and gender;

OR: Odds ratios; AOR: Adjusted odds ratios; CI: Confidence Intervals.

Multivariate logistic regressions were adjusted for sex, age, NEDS year, income groups, primary payer, hospital region, hospital location, and ED event.

$\$ \S$ Any injury visit variable does not include assault, self-harm, suicidal ideation, and multiple injuries. Sig.: ***P $<0.001 ; * * 0.001 \leq \mathrm{P}<0.01 ; * 0.01 \leq \mathrm{P}<0.05$. ns: not significant 
PhD Dissertation Chapter 3

Table 6

Type Of Visits And Mean Total ED Charges in the Pooled Study Sample Adults with and without ASD matched on age and gender 2006-2011 Nationwide Emergency Department Sample $(\mathrm{n}=102,108)$

\begin{tabular}{|c|c|c|c|c|c|}
\hline & \multicolumn{2}{|c|}{ ASD } & \multicolumn{2}{|c|}{ No ASD } & \multirow[t]{2}{*}{ Sig. } \\
\hline & Mean & SE & Mean & SE & \\
\hline Psychiatric Visit & $\$ 12,506$ & $\$ 559$ & $\$ 7,238$ & $\$ 359$ & $* * *$ \\
\hline $\mathrm{ADD} / \mathrm{ADHD}$ & $\$ 4,963$ & $\$ 1,341$ & $\$ 1,510$ & $\$ 267$ & * \\
\hline Adjustment Disorders ${ }^{\mathrm{a}}$ & $\$ 10,362$ & $\$ 3,583$ & $\$ 4,119$ & $\$ 562$ & $\mathrm{~ns}$ \\
\hline Anxiety Disorders & $\$ 5,651$ & $\$ 1,481$ & $\$ 2,217$ & $\$ 184$ & $*$ \\
\hline AUD & $\$ 14,486$ & $\$ 3,324$ & $\$ 6,462$ & $\$ 555$ & $*$ \\
\hline Bipolar Disorder & $\$ 15,576$ & $\$ 876$ & $\$ 10,136$ & $\$ 1,069$ & $* * *$ \\
\hline Depression & $\$ 10,241$ & $\$ 616$ & $\$ 5,582$ & $\$ 366$ & $* * *$ \\
\hline Intellectual Disabilities & $\$ 3,759$ & $\$ 418$ & $\$ 3,061$ & $\$ 787$ & $\mathrm{~ns}$ \\
\hline Personality Disorders & $\$ 9,078$ & $\$ 2,350$ & $\$ 3,299$ & $\$ 931$ & $*$ \\
\hline Schizophrenia & $\$ 20,336$ & $\$ 1,168$ & $\$ 12,183$ & $\$ 1,088$ & $* * *$ \\
\hline SUD & $\$ 11,581$ & $\$ 1,384$ & $\$ 8,523$ & $\$ 789$ & $*$ \\
\hline Injury Visit & $\$ 12,912$ & $\$ 715$ & $\$ 5,842$ & $\$ 345$ & $* * *$ \\
\hline Assault ${ }^{\S}$ & $\$ 8,174$ & $\$ 1,872$ & $\$ 8,836$ & $\$ 1,218$ & $\mathrm{~ns}$ \\
\hline Cut & $\$ 4,953$ & $\$ 944$ & $\$ 2,221$ & $\$ 135$ & $* *$ \\
\hline Drown $^{\mathrm{a}}$ & $\$ 17,044$ & $\$ 1,155$ & $\$ 2,534$ & $\$ 759$ & $* *$ \\
\hline Fall & $\$ 11,230$ & $\$ 934$ & $\$ 5,880$ & $\$ 475$ & $* * *$ \\
\hline Fire $^{\mathrm{a}}$ & $\$ 20,605$ & $\$ 8,333$ & $\$ 2,890$ & $\$ 558$ & $*$ \\
\hline Machinery ${ }^{\mathrm{b}}$ & $\$ 18,562$ & $\$ 0$ & $\$ 5,248$ & $\$ 528$ & $\mathrm{nc}$ \\
\hline Poison & $\$ 11,790$ & $\$ 1,252$ & $\$ 13,201$ & $\$ 1,451$ & $\mathrm{~ns}$ \\
\hline Self-harm & $\$ 13,001$ & $\$ 1,106$ & $\$ 11,560$ & $\$ 1,810$ & ns \\
\hline Struck & $\$ 3,672$ & $\$ 406$ & $\$ 3,087$ & $\$ 390$ & ns \\
\hline Suffocation $^{\mathrm{a}}$ & $\$ 53,110$ & $\$ 6,129$ & $\$ 42,061$ & $\$ 17,630$ & $\mathrm{~ns}$ \\
\hline Suicidal Ideation & $\$ 12,579$ & $\$ 701$ & $\$ 8,013$ & $\$ 481$ & $* * *$ \\
\hline Non-psychiatric Visit & $\$ 13,662$ & $\$ 681$ & $\$ 9,521$ & $\$ 454$ & $* * *$ \\
\hline Cancer & $\$ 75,352$ & $\$ 10,528$ & $\$ 67,434$ & $\$ 7,907$ & $\mathrm{~ns}$ \\
\hline Cardiovascular Disease & $\$ 39,432$ & $\$ 3,671$ & $\$ 27,181$ & $\$ 1,893$ & $* *$ \\
\hline Diabetes & $\$ 19,574$ & $\$ 2,194$ & $\$ 16,146$ & $\$ 1,955$ & $\mathrm{~ns}$ \\
\hline Epilepsy & $\$ 9,551$ & $\$ 553$ & $\$ 6,238$ & $\$ 435$ & $* * *$ \\
\hline Gastrointestinal Disease & $\$ 16,224$ & $\$ 2,263$ & $\$ 7,032$ & $\$ 568$ & $* * *$ \\
\hline Respiratory Disease & $\$ 5,431$ & $\$ 745$ & $\$ 2,455$ & $\$ 135$ & $* * *$ \\
\hline
\end{tabular}

The estimates are provided from NEDS representing ED visits with adults aged 22-64 years from the years 2006-2011; ED visits with and without ASD were matched on age and gender; ${ }^{a}$ Relative standard errors $>30 \%$. Estimates may not be precise.

${ }^{\mathrm{b}}$ Only 1 individual with ASD reported having machinery associated injury.

${ }^{\S}$ Data on assault intent was only available for the years 2009-2011.

Sig.: ***P $<0.001 ; * * 0.001 \leq \mathrm{P}<0.01 ; * 0.01 \leq \mathrm{P}<0.05$.

nc: not conclusive. Tests are not feasible because of low numbers.

ns: not significant 
PhD Dissertation Chapter 4

Study 3. The Burden of Medication Use in Adult Fee-for-Service Medicaid Enrollees with and without Autism Spectrum Disorders

Prepared by Rini Vohra

Submitted to Committee Members

Dr. Suresh Madhavan

Dr. Usha Sambamoorthi

Dr. Nilanjana Dwibedi

Dr. Claire StPeter

Dr. Susannah Poe 


\section{PhD Dissertation Chapter 4}

\section{CONTENTS}

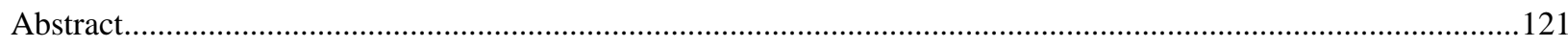

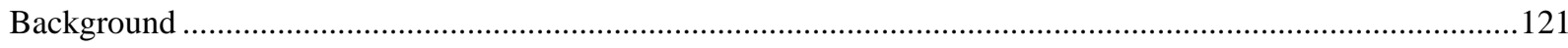

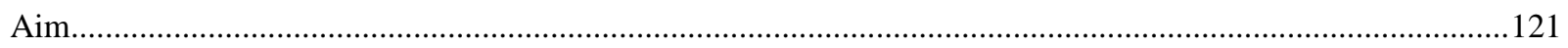

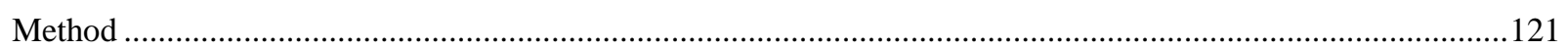

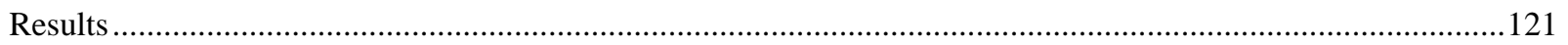

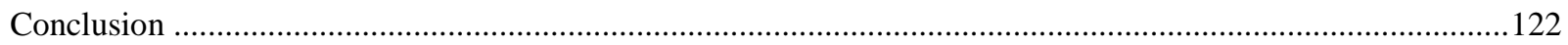

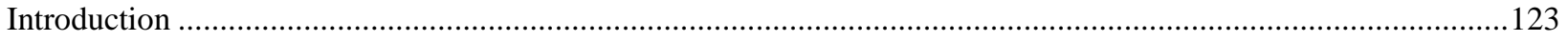

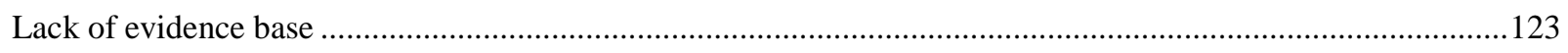

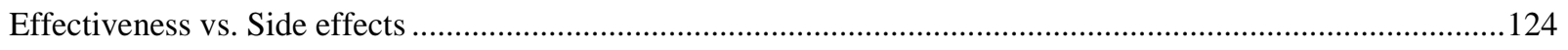

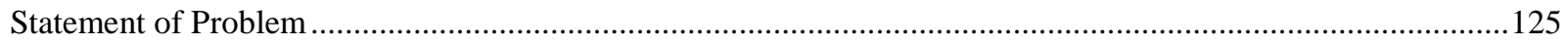

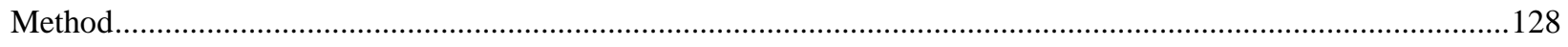

Data

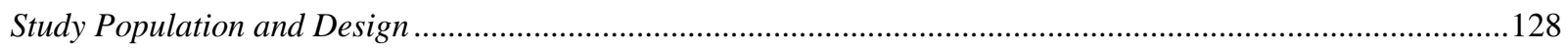

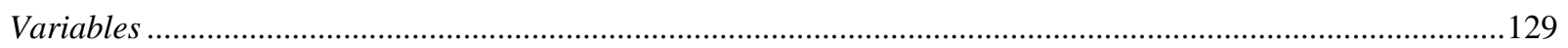

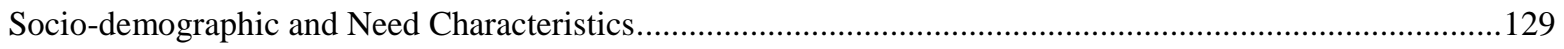

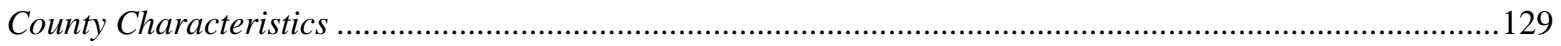

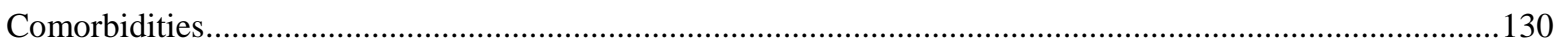

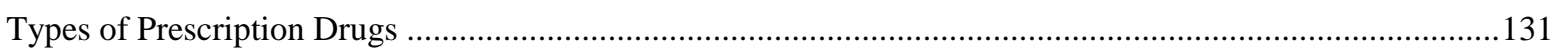

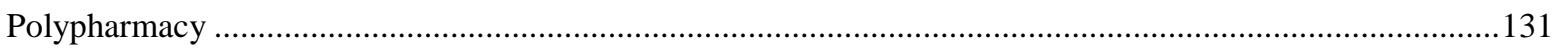

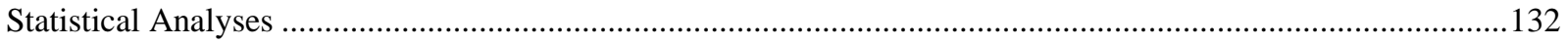

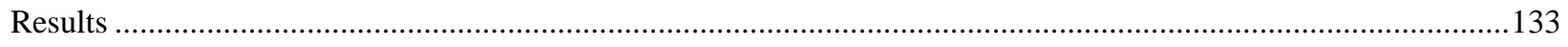

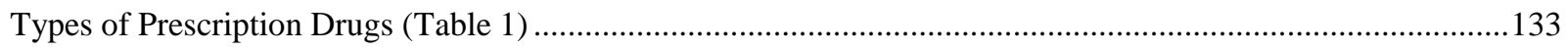

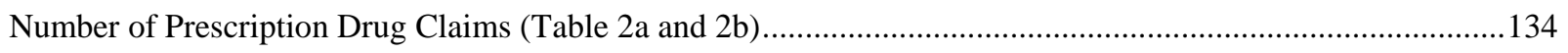

General Polypharmacy (Six or more drug classes per year; Table 1 and Table 3) ..............................................134

Psychotropic Polypharmacy (Three or more psychotropic drug classes within a 90 day period; Table 1 and 3) .136

Predictors of polypharmacy among adults with ASD (Table not shown) ……....................................................137

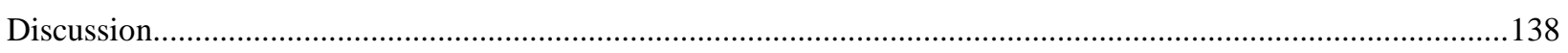

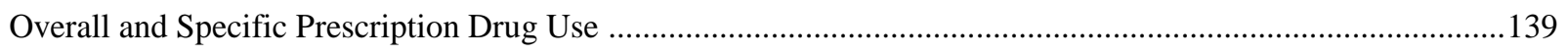

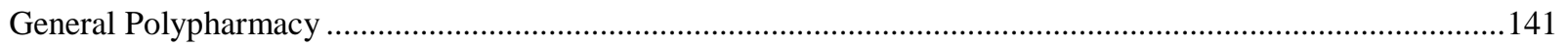

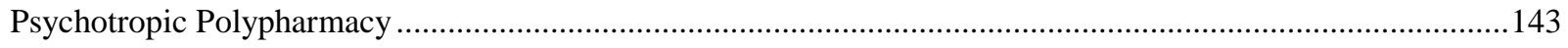

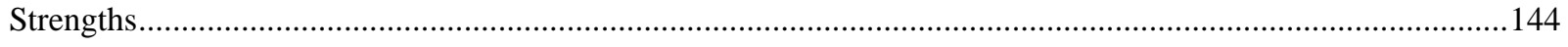

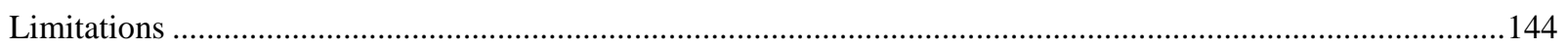

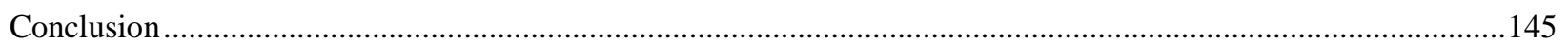

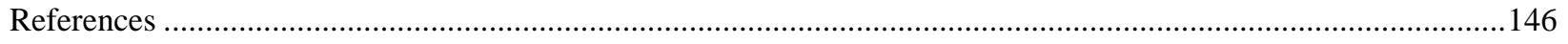

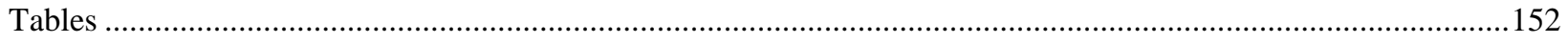




\title{
PhD Dissertation Chapter 4
}

\begin{abstract}
Background: Lack of absolute gold standard ASD treatment, approved guidelines, and strong evidence based treatments lead to greater off label prescribing with no information on the long term effects of high prescription drug use among individuals with ASD. Extent of general (six or more drug classes in a year) and psychotropic (three or more psychotropic drug classes within a period of 90 days) polypharmacy among adults with ASD has not been studied till date.
\end{abstract}

Aim: 1) To examine and compare the types of prescription drug use and rates of general and psychotropic polypharmacy among adults with and without ASD; 2) To examine predictors of polypharmacy among adults with ASD.

Method: A retrospective cross sectional study using claims data from three state Medicaid Analytic eXtract was conducted. Adults aged 22-64 years with ASD (ICD9-CM code: 299.xx) who were continuously enrolled between January 1, 2000 to December 31, 2008 were identified. ASD cases were 1:3 matched to no ASD controls by age, sex, and race using propensity score matching. Rates of different prescription drug classes, general polypharmacy, and psychotropic polypharmacy among adults with and without ASD were compared using chi-square tests. Ttests were conducted to examine the differences in mean annual number of claims for specific drug classes between adults with and without ASD. Multivariate multinomial logistic regressions were run to examine the likelihood of general and psychotropic drug polypharmacy among adults with ASD as compared to adults without ASD, after adjusting for identification year, patient socio-demographics, county characteristics, and number of comorbidities.

Results: Almost $70 \%$ of adults with ASD had more than 25 prescription drug (Rx) claim in the identification year as compared to $28 \%$ of adults without ASD. Around $85 \%$ of adults with ASD used psychotropic drugs as compared to $42 \%$ of adults without ASD. The rates of specific 


\section{PhD Dissertation Chapter 4}

psychotropic drug classes were: antipsychotics (66\% ASD vs. 20\% $\%_{\text {noASD }}$ ), anticonvulsants (59\% ASD vs. $20 \%_{\text {noASD }}$ ), antidepressants (37\% ASD vs. $26 \%_{\text {noASD }}$ ), anxiolytics/hypnotics/sedatives (21\% ASD vs. $\left.11 \%_{\text {noASD }}\right)$, and CNS stimulants (1.9\% ASD vs. $\left.1 \%_{\text {noASD }}\right)$. Around half of adults with ASD used six or more Rx classes per year as compared to one-third of adults without ASD. Among adults with ASD who used six or more Rx classes per year, the most common classes were: antipsychotics (78\%), anticonvulsants (75\%), antimicrobials (67\%), dermatologic agents (64\%), respiratory agents (59\%), and gastrointestinal agents (52\%). A substantial proportion of adults with ASD (19\%) also engaged in psychotropic polypharmacy as compared to adults without ASD (6\%). After adjusting for selected characteristics, adults with ASD were 17\% more likely and $40 \%$ more likely to engage in general and psychotropic polypharmacy, respectively, as compared to adults without ASD.

Conclusion: Prescription drug use, general polypharmacy, and psychotropic polypharmacy is significantly and substantially higher among adults with ASD than adults without ASD. Half of adults with ASD use six or more drug classes in a year and 19\% of them use three or more psychotropic drug classes within a 90 day period. Higher prescription drug use among adults with ASD was not restricted to psychotropic drugs, but extended to prescriptions alleviating other medical needs including dermatologic agents, respiratory agents, cardiovascular agents, gastrointestinal agents, antiparkinsonian agents, and many others. The study highlights considerable pharmaceuticals use by adults with ASD as well as practice of off-label prescriptions for adults with ASD by healthcare providers. Even though various clinical trials have provided with off-label options for pharmacological treatment of adults with ASD, such treatments should still be administered based on case by case analysis with due consideration for risk-benefit ratios. 


\section{PhD Dissertation Chapter 4}

\section{Introduction}

Individuals with autism spectrum disorders (ASD) often have characteristic behavioral problems such as aggression, irritability, and self- injury (Jang et al., 2011; Matson \& Rivet, 2008; Matson, Wilkins, \& Macken, 2009; Murphy et al., 2005), along with multiple medical and psychiatric comorbidities (Buie et al., 2010; Kohane et al., 2012; McElhanon et al., 2014; Simonoff et al., 2008; van Steensel et al., 2013). Management of such behavioral issues and comorbid conditions are usually pharmacological (LeClerc \& Easley, 2015) and lead to substantial medication use (Baribeau \& Anagnostou, 2014; Matson, Sipes, Fodstad, \& Fitzgerald, 2011; Myers, 2007; Volkmar et al., 1999). Till date, there are no prescription drugs that treat core ASD symptoms, but the US Food and Drug Administration approved risperidone (2006) and aripiprazole (2009) to be used primarily for symptoms of irritability, hyperactivity, and aggression associated with ASD (Warren et al., April 2011). Pharmacological treatment is clinically essential or warranted in most cases, yet there are reasons why high prescription drug use, especially psychotropic drugs as well as polypharmacy (use of more than a single class of drug) may be of concern:

\section{Lack of evidence base}

There is a lack of strong evidence for safety and efficacy of prescription drugs that are used off-label (where physicians often prescribe non-indicated medications, in a trial-and-error fashion to help manage troublesome symptoms in patients, especially if other treatments are failing) among adults with ASD (Warren et al., April 2011). Increasing number of studies are documenting the practice of off-label prescriptions by physicians to manage cases where the approved medications are either not available or not considered an adequate treatment option for individuals with ASD (Dalsgaard, Nielsen, \& Simonsen, 2013; Frazier et al., 2011b; Zito et al., 2008). A Danish study using a national registry indicated a 4.7 fold increase in prescription of 


\section{PhD Dissertation Chapter 4}

atomoxetine, methylphenidate, and dexamphetamine to children with ASD from 2003-2010

(Dalsgaard et al., 2013). This practice also extends to psychotropic drug prescriptions including selective serotonin reuptake inhibitors (SSRI's) (Baribeau \& Anagnostou, 2014) for repetitive behaviors, stimulant medications for ADHD type symptoms (Barnard-Brak, Davis, Schmidt, \& Richman, 2014) and anti-epileptic medications such as valproate for behavioral symptoms in ASD (Hirota, Veenstra-Vanderweele, Hollander, \& Kishi, 2014).

\section{Effectiveness vs. side effects}

Aripiprazole and risperidone display medium level effectiveness but are associated with substantial adverse effects (Warren et al., April 2011). (Dove et al., 2012) conducted a systematic review to find the evidence base for treatment for adults and adolescents (13-30 years) with ASD. The study concluded a moderate evidence for risperidone as a medication for controlling aggression in this population and mentioned a weak evidence for any other treatment option. However, the study also underlined a strong evidence base for adverse events associated with use of risperidone (such as sedation, obesity, and tardive dyskinesia). Risperidone is also known to cause increased appetite, fatigue, drowsiness, dizziness, and drooling in children with ASD (McCracken et al., 2002). Use of aripiprazole is associated with weight gain, sedation, and sialorrhea (Blankenship et al., 2010; Ching \& Pringsheim, 2012; M. P. Curran, 2011; Marcus et al., 2009; Owen et al., 2009). A medical chart review study (Adler et al., 2014) showed that 40\% of patients with ASD required medication adjustment despite previous trials of risperidone and aripiprazole or combinations of these together for ASD symptoms.

Other medications such as antidepressants and mood stabilizers also cause significant tolerability issues. A recent systematic review on clinical trials examining the efficacy of SSRI's (fluoxetine, fluvoxamine, fenfluramine, and citalopram) reported no significant improvements in 


\section{PhD Dissertation Chapter 4}

ASD symptoms but rather posed additional risk of harm (K. Williams et al., 2010). Use of antidepressants is also associated with behavioral activation (hyperactivity and agitation), aggression, and suicidal ideation in children and adolescents with ASD (West et al., 2009).There is a lack of robust information on long term effects of use of multiple drug classes in adults with ASD along with paucity of trials examining risk-benefit analyses of prescribing medications offlabel (Lake, Balogh, \& Lunsky, 2012; LeClerc \& Easley, 2015).

\section{Statement of Problem}

Majority of the published literature is limited and provides scattered information on overall prescription drug and polypharmacy use among individuals with ASD. In addition, studies that have examined and reported rates of prescription drug utilization, have focused primarily on pediatric or adolescent populations of ASD (Armand-Branger, Poisson, GaudoneixTaieb, \& Ramos, 2009; Buck et al., 2014b; Esbensen et al., 2009; Lake et al., 2012; Logan et al., 2012; Mandell et al., 2008a; Oswald \& Sonenklar, 2007a; Rosenberg et al., 2010a). Many of these studies among individuals with ASD indicated that increasing age was associated with greater likelihood of prescription drug use (Khanna et al., 2013; Mandell et al., 2008a; Rosenberg et al., 2010b). A report published by (Esbensen et al., 2009) on assessment of medication use at baseline and follow up period among 286 adolescents and adults with ASD (average age 21 years), showed that on an average individuals with ASD who were using 1-2 medications at baseline increased to 2-3 medications per person by the end of the follow up period. A majority of the studies examining polypharmacy among individuals with ASD are limited to psychotropic polypharmacy, which is found to be prevalent among $10-20 \%$ of children with ASD (Coury et al., 2012a; Frazier et al., 2011b; Gerhard, Chavez, Olfson, \& Crystal, 2009; 


\section{PhD Dissertation Chapter 4}

Logan et al., 2012; Mandell et al., 2008a; Oswald \& Sonenklar, 2007a; Oswald \& Sonenklar, 2007b; Spencer et al., 2013).

The current study will focus on identifying the most common therapeutic classes of drugs used by adults aged 22-64 years with and without ASD enrolled in Medicaid fee for service program. Since recently it has been shown that adults with ASD have significantly higher rates of medical conditions such as metabolic disorders, immune system dysfunctions, gastrointestinal issues, and eating disorders as compared to adults without ASD (Croen et al., 2015), it is critical to examine the burden of other medications in this population. A few previous studies have examined specific types of prescription drug use (mostly psychotropic) among younger and older youth with ASD (Buck et al., 2014a; Esbensen et al., 2009; Khanna et al., 2013). (Esbensen et al., 2009) focused on the use of psychotropic (such as antipsychotics and anti-depressants) and non-psychotropic medications (such as anti lipimic, GI medications, and other drugs) among adolescents and adults with ASD (average age 21 years) via longitudinal (4.5 years) contingency analyses and found that both types of prescription drug use among adults with ASD is very high. Using literature support of such studies, we hypothesize that adults with ASD will have a higher use of psychotropic as well as other therapeutic drug classes as compared to adults without ASD.

The current study will also compare the rates of general polypharmacy (use of six or more unique classes of prescription drugs in a year) and psychotropic polypharmacy (concurrent use of 3 or more psychotropic drug classes within a 90 day period after the first prescription fill for a psychotropic drug) among adults with and without ASD. We expect greater rates of general and psychotropic polypharmacy among adults with ASD as compared to adults without ASD. This may be due to paucity of clinical trials for most prescription drugs among adults with ASD, lack of training of healthcare providers in terms of treatment guidelines (Bruder et al., 


\section{PhD Dissertation Chapter 4}

2012; Golnik et al., 2009; Heidgerken et al., 2005; Oskoui \& Wolfson, 2012), greater risk for adults with ASD to use psychotropic medications than their younger counterparts (Esbensen et al., 2009; Khanna et al., 2013), and low likelihood of discontinuation of a medication once started at a younger age (Esbensen et al., 2009).

Our study will use three state Medicaid administrative claims data (IL, NY, and TX; 2000-2008) to determine the rates of overall prescription drug use, specific prescription drug use, general polypharmacy, and psychotropic polypharmacy among adults with and without ASD in a 1:3 matched cohort (age, gender, and race). Although, prescription drug coverage is an optional benefit under federal Medicaid law, all state Medicaid programs provide coverage for outpatient pharmacy prescription drugs to eligible individuals. Medicaid plays a significant role in prescription drug coverage for individuals with mental health and substance abuse issues (Schubart et al. 2014). Many studies have utilized Medicaid claims databases to examine the rates of psychotropic polypharmacy among children with ASD (Frazier et al., 2011a; Logan et al., 2012; Mandell et al., 2008b). Thus, using this database will help provide a comprehensive understanding of prescription drug use among adults with ASD. 


\section{PhD Dissertation Chapter 4}

\section{Method}

Data

Administrative health insurance claims data from three state Medicaid programs (IL, NY, and TX) were extracted from the 2000-2008 Centers for Medicare and Medicaid Services Medicaid analytic eXtract (MAX) data files. We selected IL, NY, and TX because of lower managed care penetration rates and greater fee-for-service enrollment during the study period (The Henry J Kaiser Family Foundation, 2013). The MAX files provide diagnosis codes, service use, demographic characteristics, and state of residence. Separate files received from CMS can be linked based on beneficiaries' unique identification number.

For the current study we used the personal summary file (provides information on Medicaid eligibility, enrollment type, and demographics), other therapy file (provides information on two possible diagnoses, utilization of outpatient services such as clinical services, physician services, procedures, home health, and lab services), inpatient file (provides information on nine possible diagnoses, hospitalizations, procedures, and length of stays), and the prescription drug file (national drug codes (NDC), prescription fill date, and days of supply). County level variables were obtained from the area resource file (ARF) (Quality Resource Systems, 2006) which provides information on type of health facilities, number and type of health professions, resource scarcity measures, health status, economic activities, health training programs, and socioeconomic and environmental characteristics. The Medicaid files were linked with ARF files with a five digit county identification variable.

\section{Study Population and Design}

A retrospective cross-sectional matched cohort study among adults aged 22-64 years with and without ASD was conducted. Adults with any ASD (at least 1 inpatient or 2 outpatient 


\section{PhD Dissertation Chapter 4}

claims on separate service dates with an ICD9-CM code: 299.xx in any position) and continuously enrolled in a fee for service (FFS) program from 2000-2008 were matched (1:3) with adults without ASD by age, gender, and race using propensity score matching method with GREEDY (1:8) algorithm. Adults without ASD were selected only if they had at two least medical claims on separate dates of service (either inpatient or outpatient). We excluded enrollees who were: a) dual Medicaid/Medicare eligible; b) Managed care enrollees; c) dead; and d) did not have a medical claim (outpatient/inpatient services). Due to the low number of FFS enrolled adults with ASD in the year 2008, their data was merged with the adults with ASD identified in the year 2007. The baseline characteristics (patient's socio-demographic information, comorbidities, drug use, and polypharmacy) were extracted from the identification year (first medical claim year with any ASD diagnosis).

\section{Variables}

Socio-demographic Characteristics

Age (22-40 and 41-64 years), sex (male and female), race (White, African-American, and Other), state (IL, NY, and TX), identification cohort (before and after 2004), and Medicaid eligibility by cash and medical needs (yes/no) were extracted from the MAX personal summary file.

\section{County Characteristics}

We used various contextual variables from the ARF file such as county metro status (urban/rural), primary care shortage area (yes/no), mental health specialist shortage area (yes/no), psychiatrist density (high/low), median household income level (quartiles: q1, q2, q3, and q4), and high school education and above density which is a measure of county education status (quartiles: q1, q2, q3, and q4). These were used as proxy measures of access to services. 


\section{PhD Dissertation Chapter 4}

\section{Comorbidities}

We included both number (none, one, two, three or more) and types of comorbid conditions (either 1 inpatient or 2 outpatient claims on separate service dates with a diagnosis in any position for a psychiatric or a non-psychiatric disorder). Variable representing number of comorbidities was created as a sum of any psychiatric disorder, any non-psychiatric disorder, and other relevant health conditions which were found to be of significant prevalence in both ASD and no ASD groups (epilepsy, thyroid disorders, lipid metabolism disorders, pancreatic disorders, fluid and electrolyte disorders, jaw and teeth disorders, genitourinary disorders, skin disorders, and infections).

We used the single level clinical classification software (CCS) provided by Agency for Healthcare Research and Quality (https://www.hcup us.ahrq.gov/toolssoftware/CCS/AppendixASingleDX.txt) to categorize diagnosis codes to clinically meaningful categories. A psychiatric comorbidity included: adjustment disorders (CCS code: 650), alcohol/substance use disorders (AUD; CCS code: 660,661), anxiety disorders (CCS code: 651), attention deficit disorders \& conduct behavior disorders (ADD; CCS code: 652), developmental disorders (CCS code: 654), mood disorders including depressive disorders and bipolar disorder (CCS code: 6571,6572), personality disorders (CCS code: 658), and schizophrenia \& other psychotic disorders (CCS code: 659). A non-psychiatric comorbidity included: cancer (CCS code: 11- 37, 39-43, 46, 47) cardiovascular disease (CCS code: 96-108), diabetes (CCS code: 49, 50), muscoskeletal disorders (CCS code: 201-212), gastrointestinal disease (CCS code: 138-151, 153-155), and respiratory disease (CCS code: 125-128, 132-134). 


\section{PhD Dissertation Chapter 4}

\section{Types of Prescription Drugs}

The study examined use of any prescription drug class as well as the use of specific drug classes. We examined the proportion of adults using specific drug classes as well as the mean number of Rx claims per year for the following drug classes: respiratory agents (antihistamines, bronchodilators, inhaled corticosteroids, respiratory inhalants, leukotriene modifiers etc.), antimicrobials (amebicides, anthelmintics, antifungals, antibiotics, antiviral, antituberculosis etc.) anticancer (antineoplastics, radiologics, and biologicals), immunologics (immunoglobulins and immune-suppressives/stimulants), cardiovascular agents (antiadrenergics, ACE inhibitors, beta blockers, antiarrythmics, diuretics, vasodilators, etc.) analgesics, blood modifiers (antiplatelet agents, anticoagulants, and other coagulants) hormone modifiers/steroids (glucocorticoids, sex hormones, thyroid hormones, other adrenal corticoids, etc.), statins/other antihyperlipidemics, antidiabetics, dermatologic agents, alternative medicine/nutritional supplements, gastrointestinal agents, CNS agents (excluding stimulants), antiparkinsonian agents, and other metabolic agents. These agents were identified using drug database from Multum® Lexicon 2014 (Cerner Multum, Inc., Denver, CO), which provides national drug codes or NDC's (eleven digit code). Such NDC's represents unique prescription drugs that are assigned drug categories which can be classified further into meaningful therapeutic drug classes and can be linked with the MAX pharmacy file.

\section{Polypharmacy}

We defined general polypharmacy (yes, no, no Rx claim) as use of six or more unique

classes of prescription drugs in a year. General polypharmacy may include but is not restricted to prescription drug classes such as antipsychotics, antidiabetics, antihyperlipidemics, antineoplastics, antibiotics, gastrointestinal agents, respiratory agents, and many other classes of 


\section{PhD Dissertation Chapter 4}

drugs. The psychotropic drug polypharmacy (yes, no, no Rx claim) was defined as use of three or more unique classes of psychotropic drugs within a 90 day period after the first occurring prescription fill date of any psychotropic drug. A similar definition of psychotropic polypharmacy in claims database analyses has been previously used by (Spencer et al., 2013) among children with ASD. The psychotropic drug classes in the current study included: antidepressants, antipsychotics, anticonvulsants, CNS stimulants, and anxiolytics/sedatives/hypnotics. For converting the national drug codes to therapeutic drug classes, we used drug database from Multum® Lexicon 2014 (Cerner Multum, Inc., Denver, CO) and Healthcare Effectiveness Data and Information Set (HEDIS) NDC 2015 lists (National Committee for Quality Assurance, 2015).

\section{Statistical Analyses}

Bivariate analyses such as chi-square tests of associations for categorical variables and ttests for continuous variables were conducted to assess the sub-group and mean differences between adults with and without ASD. To examine and compare the likelihood of general and psychotropic polypharmacy we conducted a multinomial logistic regression among adults with and without ASD. For predictors of polypharmacy within adults with ASD, the multinomial variable was converted to a binary indicator (polypharmacy vs. no polypharmacy). Numbers, percentages, means and standard deviations, adjusted odds ratios (AOR) along with 95\% confidence intervals (CI) are reported. Mean number of Rx claims per year for each therapeutic drug class are presented for the entire sample as well as among users only. Analyses were adjusted for socio-demographics, county characteristics, and number of comorbidities. Findings with cell sizes that were 11 or less are suppressed in accordance with CMS data user agreement. All analyses were conducted using SAS v9.4. 


\section{PhD Dissertation Chapter 4}

\section{Results}

Study sample had 1,772 adults with ASD, most of them being male (71\%), aged 22-40 years $(86 \%)$, and under a cash assistance program (82\%). Rates of psychiatric (81\% ASD vs. $\left.41 \%_{\text {noASD }}\right)$ and non-psychiatric comorbidity (40\% ASD vs. $\left.45 \%_{\text {noASD }}\right)$ were significantly higher among adults with ASD as compared to adults without ASD. Around 4\% of adults with ASD were Medicaid eligible by medical needs as compared to $17 \%$ of adults without ASD. About $82 \%$ of adults with ASD were Medicaid eligible by cash assistance as compared to $60 \%$ of adults without ASD.

\section{Types of Prescription Drugs (Table 1)}

Almost $95 \%$ of adults with ASD had at least one Rx claim in the identification year as compared to $87 \%$ of adults without ASD. A significantly greater proportion of adults with ASD used dermatologic agents (48\% ASD vs. 32\% noASD), respiratory agents (38\% ASD vs. 34\% noASD), gastrointestinal agents (32\% ASD vs. 23\% noASD), alternative medicine/nutritional supplements

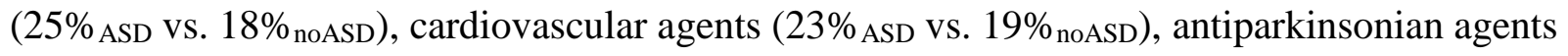
(23\% ASD vs. $10 \%$ noASD), statins/other antihyperlipidemics (7.3\% ASD vs. 5\% noASD), immunologics ( $2 \%_{\text {ASD }}$ vs. $\left.0.7 \%_{\text {noASD }}\right)$, and other metabolic agents (1.4\% ASD vs. $\left.1.1 \%_{\text {noASD }}\right)$. Any psychotropic drug use was also significantly higher among adults with ASD (85\%) as compared to adults without ASD (42\%). The rates of specific psychotropic drug use among adults with and without ASD were: antidepressants (37\% ASD vs. $26 \%$ noASD), antipsychotics (66\% ASD vs. $20 \%$ noASD), anticonvulsants (59\% ASD vs. $\left.20 \%_{\text {noASD }}\right)$, CNS stimulants (1.9\% ASD vs. $\left.1 \%_{\text {noASD }}\right)$, and anxiolytics/hypnotics/sedatives (21\% ASD vs. $11 \%$ noASD). 


\section{PhD Dissertation Chapter 4}

Number of Prescription Drug Claims (Table $2 a$ and $2 b$ )

On the average, adults with ASD had a significantly higher number of prescription drug claims per year (48 ASD vs. $21_{\text {noASD }}$ claims). The mean number of Rx claims per year for some specific therapeutic classes were also significantly higher in the ASD group as compared to no ASD group. Examples of such therapeutic classes were: gastrointestinal agents ( $4_{\text {ASD }}$ vs. $1_{\text {noASD }}$ claims), antiparkinsonian agents ( $2_{\mathrm{ASD}}$ vs. $0.5_{\mathrm{noASD}}$ claims), dermatologic agents ( $3_{\mathrm{ASD}}$ vs. $1_{\mathrm{noASD}}$ claims), respiratory agents ( $3_{\mathrm{ASD}} \mathrm{vs}$. $2_{\mathrm{noASD}}$ claims), alternative medicines/nutritional supplements ( $2_{\mathrm{ASD}}$ vs. $1_{\text {noASD }}$ claims), hormone modifiers/steroids ( $2_{\mathrm{ASD}}$ vs. $1_{\text {noASD }}$ claims), cardiovascular agents ( $3_{\mathrm{ASD}}$ vs. $2_{\text {noASD }}$ claims), statins/other antihyperlipidemics ( $1_{\mathrm{ASD}}$ vs. $0.3_{\text {noASD }}$ claim), and other metabolic agents ( $0.15_{\text {ASD }}$ vs. $0.05_{\text {noASD }}$ claims). The mean number of psychotropic drug claims per year for adults with ASD were four times the number of psychotropic drug claims for adults without ASD (25 ASD vs. $6_{\text {noASD }}$ claims). The difference in mean number of psychotropic drug claims between adults with and without ASD were highest for anticonvulsants ( $10_{\text {ASD }}$ vs. $1_{\text {noASD }}$ claims), followed by antipsychotics ( $10_{\mathrm{ASD}}$ vs. $2_{\text {noASD }}$ claims), antidepressants ( $4_{\mathrm{ASD}} \mathrm{vs}$. $2_{\text {noASD }}$ claims), anxiolytics/sedatives/hypnotics ( $1_{\mathrm{ASD}}$ vs. $0.5_{\text {noASD }}$ claims), and CNS stimulants (0.1 ASD vs. $0.05_{\text {noASD }}$ claims). Among users of specific drug classes, a similar pattern of higher average number of claims was observed for adults with ASD as compared to adults without ASD (Table 2b).

General Polypharmacy (Six or more drug classes per year; Table 1 and Table 3)

The rate of general polypharmacy was $48 \%$ among adults with ASD as compared to $32 \%$ among adults without ASD. The most common drug classes used among adults with ASD who used six or more drug classes in a year were: antipsychotics (78\%), anticonvulsants (75\%), antimicrobials (67\%), dermatologic agents (64\%), respiratory agents (59\%), and gastrointestinal 


\section{PhD Dissertation Chapter 4}

agents (52\%). The most common drug classes used among adults without ASD who used six or more drug classes in a year were: antimicrobials (84\%), analgesics (77\%), respiratory agents (67\%), dermatologic agents (60\%), gastrointestinal agents (52\%), and antidepressants (50\%). In an adjusted logistic regression analyses, adults with ASD were more likely to use six or more drug classes in a year as compared to adults without $\mathrm{ASD}(\mathrm{AOR}=1.17,95 \% \mathrm{CI}=1.03-1.33)$, even after adjusting for the number of comorbidities.

The number of adults with ASD with general polypharmacy increased significantly and consistently from 2000 (42\%) to 2007 (64\%). Significantly greater proportion of females with ASD (55\%) used six or more drug classes in a year as compared to males with ASD (45\%). Higher general polypharmacy rates for adults with ASD were observed in 41-64 age group (46\%) as compared to the $22-40$ age group and Whites (54\%) as compared to African Americans (47\%) and other race (44\%). Adults with ASD residing in counties with primary care provider shortage (47\%) and mental health specialist shortage (47\%) had lower rates of general polypharmacy as compared to adults with ASD residing in counties with no workforce shortage. Around 52\% of adults with ASD and a psychiatric comorbidity used six or more drug classes in a year as compared to $32 \%$ of adults with ASD but no psychiatric comorbidity. The highest use of general polypharmacy among adults with ASD was seen for those who had personality disorders (80\%), schizophrenia (66\%), and mood disorders (65\%). Adults with ASD and a nonpsychiatric disorder also had significantly high rates of general polypharmacy (68\%). Highest rates of general polypharmacy among adults with ASD were observed for those who had a gastrointestinal disorder (78\%), cardiovascular/cerebrovascular disorders (74\%), respiratory disorder (71\%), muscoskeletal disorders (68\%), and diabetes (64\%). Number of comorbidities was also associated with greater general polypharmacy rates among adults with ASD, where 


\section{PhD Dissertation Chapter 4}

$65 \%$ of adults with three or more comorbid conditions, $41 \%$ of adults with two comorbid conditions, and $27 \%$ of adults with one comorbid condition had general polypharmacy. Around $21 \%$ of adults with ASD with no comorbidity had general polypharmacy.

Psychotropic Polypharmacy (Three or more psychotropic drug classes within a 90 day period; Table 1 and 3)

Psychotropic polypharmacy was prevalent among $19 \%$ of adults with ASD as compared to $6 \%$ of adults without ASD. Adults with ASD were also more likely to use three or more psychotropic drug classes in a year as compared to adults without ASD in a multivariate logistic regression analyses $(\mathrm{AOR}=1.40,95 \% \mathrm{CI}=1.17-1.68)$. Among adults with $\mathrm{ASD}$, White race had significantly greater psychotropic polypharmacy rate as compared to African American and Other race. Adults with ASD and a psychiatric comorbidity such as adjustment disorders (26\%), mood disorders (31\%), and schizophrenia (32\%) had significantly higher rates of psychotropic polypharmacy. A little more than $24 \%$ of adults with ASD and epilepsy had psychotropic polypharmacy. The number of comorbidities was also associated with greater psychotropic polypharmacy rates among adults with ASD, where $24 \%$ of adults with three or more comorbid conditions, $17 \%$ of adults with two comorbid conditions, and $15 \%$ of adults with one comorbid condition used three or more psychotropic drug classes within a 90 day period. Around $12 \%$ of adults with ASD with no comorbidity used psychotropic polypharmacy. 


\section{PhD Dissertation Chapter 4}

Predictors of polypharmacy among adults with ASD (Table not shown)

General Polypharmacy was significantly more likely to be prevalent among adults with ASD after 2004 as compared to before $2004(\mathrm{AOR}=1.30,95 \% \mathrm{CI}=1.01-1.68)$. Male adults with ASD were significantly less likely to use six or more drug classes per year as compared to female adults with ASD (AOR $=0.63,95 \% \mathrm{CI}=0.50-0.80)$. Adults with ASD residing in counties with lower education status were less likely to have general polypharmacy as compared to adults with ASD residing in counties with higher education status (q1 vs. q4; AOR $=0.43$, $95 \% \mathrm{CI}=0.27-0.66)$. Adults with ASD who were either African American $(\mathrm{AOR}=0.50,95 \%$ $\mathrm{CI}=0.34-0.72)$ and other race $(\mathrm{AOR}=0.71,95 \% \mathrm{CI}=0.53-0.96)$ were significantly less likely to have psychotropic polypharmacy as compared to Whites. The number of comorbidities was strongly associated with greater likelihood of both general and psychotropic polypharmacy. Adults with ASD and three or more comorbidities were 12 times likely to use general polypharmacy $(\mathrm{AOR}=11.14,95 \% \mathrm{CI}=7.05-17.6)$ and three times likely to use psychotropic polypharmacy $(\mathrm{AOR}=3.03,95 \% \mathrm{CI}=1.76-5.22)$ as compared to adults with ASD but no comorbidity. 


\section{PhD Dissertation Chapter 4}

\section{Discussion}

The burden of prescription drug use among Medicaid enrolled adults with ASD is substantially and significantly higher than adults without ASD. The higher medication burden was not only restricted to greater proportion of adults with ASD using a prescription drug as compared to adults without ASD, but also extended to higher number of claims for prescription drugs. Adults with ASD use considerable number of prescriptions drugs that focus on psychiatric symptoms such as antipsychotics, antidepressants, anxiolytics/sedatives, as well as prescription drugs that alleviate medical issues including respiratory agents, gastrointestinal agents, cardiovascular agents, statins, and many others. Another notable finding of this study was that adults with ASD are more likely to use multiple drug classes as compared to adults without ASD. Almost half of the adults with ASD used six or more drug classes in a year and $19 \%$ of adults with ASD used three or more psychotropic drug classes within a 90 day period as compared to adults without ASD.

With the rising concerns of off-label use and potential risks associated with multiple drug use among individuals with ASD (LeClerc \& Easley, 2015), our study provides essential and timely information on prescription practices and the patterns of drug utilization among adults with ASD. Various studies in the past have focused on examining extent of medication use and polypharmacy among children and adolescents with ASD (Baribeau \& Anagnostou, 2014; Barnard-Brak et al., 2014; Buck et al., 2014b; Coury et al., 2012a; Frazier et al., 2011b; Hirota et al., 2014; Logan et al., 2012; Oswald \& Sonenklar, 2007a; Schubart, Camacho, \& Leslie, 2014; Spencer et al., 2013), but this study is the first large sample study using real world data which has established rates of prescription drug use, types of prescription drugs, and the extent of 


\section{PhD Dissertation Chapter 4}

polypharmacy among adults with ASD as compared to a age, gender, and race matched sample of adults without ASD.

\section{Overall and Specific Prescription Drug Use}

Almost $95 \%$ of adults with ASD had at least one prescription drug claim in a year as compared to $87 \%$ of adults without ASD. A large majority of adults with ASD (85\%) used a psychotropic drug which was significantly higher as compared to adults without ASD (42\%). Within psychotropic drugs, the most common drug classes used by adults with ASD were antipsychotics (66\%), followed by anticonvulsants (59\%), and antidepressants (37\%). As compared to previous studies (Esbensen et al., 2009; Lake et al., 2012) on psychotropic medication and polypharmacy use among adults with ASD, our psychotropic drug use was much higher than their rates of $64 \%$ (Lake et al. 2012) and 57\% (Esbensen et al. 2009). Unlike the study by (Esbensen et al., 2009) which focused on a younger age group of 10-48 years old with ASD, our study included adults up till 64 years of age which could have been the reason for elevated rates of the medication use. The study by (Lake et al., 2012), even though informative, was restricted by the clinical settings, staff reports of medication use, and a low sample size which may not be representative of community dwelling adults with ASD. Nonetheless, the psychotropic drug use rate in our study was much higher than rates among children with ASD (27-64\%), as observed in previous studies (Coury et al., 2012b; Khanna et al., 2013; Oswald \& Sonenklar, 2007a; Spencer et al., 2013).

Other than psychotropic drug use, this study also indicates that significantly higher proportion of adults with ASD use non-psychotropic drugs such as dermatologic agents (48\%), respiratory agents (38\%), gastrointestinal agents (32\%), alternative medicine/nutritional supplements (25\%), cardiovascular agents (23\%), antiparkinsonian agents (23\%), and 


\section{PhD Dissertation Chapter 4}

statins/other antihyperlipidemics (7.3\%) as compared to adults without ASD. This is a critical finding, since most of the literature in the past focused on psychiatric needs of adults with ASD and utilization of psychotropic drugs (Baribeau \& Anagnostou, 2014; Barnard-Brak et al., 2014; Buck et al., 2014b; Coury et al., 2012a; Frazier et al., 2011b; Hirota et al., 2014; Logan et al., 2012; Oswald \& Sonenklar, 2007a; Schubart et al., 2014; Spencer et al., 2013).

A recent study by (Croen et al., 2015) using claims data from the Northern California Kaiser Permanente program showed a considerable and higher prevalence of non-psychiatric comorbidities such as immune conditions, gastrointestinal disorders, dyslipidemia, hypertension, and diabetes among adults with ASD as compared to adults without ASD. In fact, adults with ASD also had a higher prevalence of rare disorders such as stroke and Parkinsonism. Recently, a study by (Starkstein, Gellar, Parlier, Payne, \& Piven, 2015) showed a 20\% prevalence of Parkinsonism among a small sample of middle aged and older adults (49 years and above) with ASD. (Hollander, Wang, Braun, \& Marsh, 2009) published a review on links between Parkinsonism and ASD which may have overlapping phenomenology such as repetitive behaviors and a common underlying involvement of the basal ganglia leading to symptoms that require similar medication management. Thus, our study findings support and extend the findings of Croen et al. (2015), underlining that adults with ASD have similar or higher nonpsychiatric needs which transforms into higher prescription drug use as well. Use of additional prescription drugs other than psychotropic drugs may be of bigger concern in a scenario where numerous medications are used off-label with no information on their benefit-risk ratios, limited data on beneficial effects of psychotropic drugs other than risperidone and aripiprazole (Warren et al., April 2011), and lack of well-designed clinical trials with large cohorts of adult ASD population (LeClerc \& Easley, 2015). 


\section{PhD Dissertation Chapter 4}

Adults with ASD also had a significantly greater mean number of drug claims per year. For example, the mean number of claims per year for gastrointestinal agents and statins/antihyperlipidemics among adults with ASD were four times and almost three times, as that for adults without ASD respectively. Adults with ASD also had four times the mean number of drug claims for a psychotropic drug as compared to adults without ASD. For some drug classes such as anticonvulsants, the mean number of claims per year were as high as 11 claims per year for adults with ASD as compared to a mean of 2 claims per year for adults without ASD. Such findings were consistent when restricted to users only, where adults with ASD still had higher mean number of claims in a year for most drug classes. Thus, adults with ASD may tend to use greater quantity of drugs in a year and may have considerably higher prescription drug needs and lead to greater prescription drug costs. (Croen et al., 2006) found that on an average Medicaid enrolled children with ASD have six drug prescriptions per year, and our study shows a rate eight times higher of 48 prescription encounters per year among adults with ASD. These results support findings from a study by (Esbensen et al., 2009) which found that young adults are less likely to discontinue medications and more likely to add medications over the course of the ASD treatment as they age. Such high prescription use among adults with ASD should be addressed by healthcare providers, policymakers, and state health insurance programs to determine their use justification and effectiveness.

\section{General Polypharmacy}

In our study cohort, almost half of the adults with ASD used six or more drug classes in a year as compared to one-third of adults without ASD. One of the interesting findings in our study was the consistent increase ( $22 \%$ rise) in the proportion of adults with ASD with general polypharmacy from 2000 to 2007. The past decade was a critical transition period for ASD 


\section{PhD Dissertation Chapter 4}

awareness and better diagnoses (Autism Speaks, 2015), better Medicaid policies for prescription drug coverage (Arjun et al., 2011; Mauch et al., 2011) for individuals with mental health issues such as ASD, and increased mental health workforce could have been associated with better access to medications which may have resulted in number of individuals getting access to prescription drugs. Adults with ASD living in counties with workforce shortage of primary care and mental health specialty providers had lower rates of general polypharmacy as compared to those living in counties with no workforce shortage, underlining the important role of healthcare providers in access to prescription drugs for adults with ASD and emphasizing the need for better pharmacological guidelines to help providers to care for adults with ASD, and reduce excess prescription drug use whenever risks outweigh benefits.

Among predictors of general polypharmacy for adults with ASD, female gender and older age was significantly associated with greater rates as compared to male gender and younger age group respectively. Even though not fully established, a few studies have shown that females with ASD are less likely to have repetitive behaviors but have a higher prevalence of other disorders such as intellectual disabilities, anxiety, schizophrenia, depression, bipolar disorders, cancer, diabetes, and some neurological disorders, putting them at a greater risk of using multiple classes of drugs (Croen et al., 2015; Rubenstein, Wiggins, \& Lee, 2015). More than half of the adults with ASD and a psychiatric comorbidity used six or more drug classes in a year and the highest general polypharmacy rates were observed among those with comorbid schizophrenia (66\%) and mood disorders (65\%). Adults with ASD and a non-psychiatric comorbidity were also highly likely to use general polypharmacy (68\%), with those with gastrointestinal disorders being associated with the highest rate of general polypharmacy at 78\%, followed by 


\section{PhD Dissertation Chapter 4}

cardiovascular/cerebrovascular disorders (74\%), and respiratory disorders $(71 \%)$. Around $21 \%$ of adults with ASD but no comorbidity used six or more drug classes per year.

\section{Psychotropic Polypharmacy}

Psychotropic polypharmacy was observed among 19\% of adults with ASD as compared to only $6 \%$ of adults without ASD. This rate of psychotropic polypharmacy was lower than that reported by (Lake et al., 2012) of 29\%. This may be because we defined psychotropic polypharmacy as use of three or more drug classes within a period of 90 days after the first prescription fill. Nevertheless, the rate of $20 \%$ was still higher as that reported by studies among children with ASD (Coury et al., 2012b; Spencer et al., 2013). Adults with ASD who had adjustment disorders (26\%), ADD/ADHD (30\%), mood disorders (31\%), schizophrenia (32\%), and epilepsy (24\%) had greater psychotropic polypharmacy rates as compared to adults with ASD but no respective comorbidities. Around $12 \%$ of adults with ASD but no comorbidity used three or more psychotropic drugs in a 90 day period. This finding shows that physicians frequently follow off label prescription practices without strong evidence for drugs such as selective serotonin reuptake inhibitors and drugs such as memantine for improvements in autistic symptoms such as irritability, hyperactivity, and aggression (Croen et al., 2015; Rubenstein et al., 2015). In addition, use of multiple classes of drugs at one time also increases the risks of adverse events such as tardive dyskinesia, a strong and lethal consequence of risperidone therapy, as well as cause weight gain, gastrointestinal issues, and increased self-injurious and extreme behaviors that further worsen ASD symptoms (Myers, 2007). This worsening of symptoms may contribute to greater use of emergency departments and hospitalizations and higher costs of healthcare for adults with ASD (Kato et al., 2013; Myer et al., 2013; Myers, 2007). 


\section{PhD Dissertation Chapter 4}

\section{Strengths}

Our study is the first study to examine the extent of prescription drug use and polypharmacy rates among adults with ASD using a large population based setting and real world data. We used three state Medicaid database, a program that provides extensive pharmacy services and home and community based waivers to individuals with ASD (Ruble et al., 2005; Semansky, Xie, \& Mandell, 2011b). Unlike a few other studies that have provided estimates of polypharmacy among very restricted number of adults with ASD in clinical settings, our study utilized a large sample of adults with ASD and provide estimates that are not restricted to psychotropic polypharmacy use. The current study is also the first study to estimate the average number of claims per year for specific therapeutic classes among adults with ASD and compare the rates with a matched sample of adults without ASD. State Medicaid programs have made consistent efforts to reduce prescription drug use and costs since early 2000's (National Conference of State Legislatures, 2014) and our study highlights that adults with ASD, despite having many medical necessities, have substantial and/or off label use of prescription drugs as compared to other Medicaid beneficiaries.

\section{Limitations}

Despite the various strengths, our study was limited in some ways. Our study period was restricted and definition of ASD and many drug related policies/rebate programs have changed after 2008. In addition, the list of drug classes coded using NDCs may not have been exhaustive and some drugs which are provided in intravenous form (e.g. anticancer therapies) or via inpatient pharmacy are not reflected in the findings. We required adults with ASD to be continuously enrolled during the study period to allow capturing their full prescription drug utilization date, which could have excluded many individuals with sporadic Medicaid 


\section{PhD Dissertation Chapter 4}

enrollment. We did not match the ASD cases and no ASD controls on their enrollment period, which could have created certain baseline differences in the two groups. A majority of adults in the study were eligible by cash assistance indicating that our sample was primarily from low income and/or had high needs, thus rates of drug use may be overrepresented for both ASD and no ASD groups. Definitions of polypharmacy have varied substantially across studies but we used definitions that were most relevant to our population of interest. The rate of psychotropic polypharmacy may have been underestimated because we did not include patients with a psychotropic polypharmacy with any 90 day period in the year, rather restricted it to 90 days after the first prescription fill for a psychotropic drug.

\section{Conclusion}

Prescription drug, general polypharmacy, and psychotropic polypharmacy use among adults with ASD is significantly and substantially higher than adults without ASD. Half of adults with ASD use six or more drug classes in a year and almost $20 \%$ of them use three or more psychotropic drug classes within a 90 day period. Higher prescription drug use among adults with ASD was not restricted to psychotropic drugs, but extended to prescriptions alleviating other medical needs including cardiovascular agents, statins, immunologics, gastrointestinal agents, antiparkinsonian agents, and many others. The study highlights considerable pharmaceutical needs of adults with ASD as well as the practice of off-label prescription use for adults with ASD by healthcare providers. Even though various clinical trials have provided offlabel options for pharmacological treatment of adults with ASD, such treatments should still be administered based on case by case analysis with due consideration for risk-benefit ratios. 


\section{PhD Dissertation Chapter 4}

\section{References}

Adler, B. A., Wink, L. K., Early, M., Shaffer, R., Minshawi, N., McDougle, C. J., \& Erickson, C. A. (2014). Drugrefractory aggression, self-injurious behavior, and severe tantrums in autism spectrum disorders: A chart review study. Autism: The International Journal of Research and Practice, doi:1362361314524641 [pii]

Arjun, L., Hope, C., Guyer, J., \& Alker, J. (2011). Medicaid and its role for children and youth with special health care needs (CYSHCN): A family perspective. (). Georgetown University, Health Policy Institute: Center for Children and Families/Family Voices.

Armand-Branger, S., Poisson, N., Gaudoneix-Taieb, M., \& Ramos, O. (2009). Pharmaco-therapeutic evaluation of medical treatments of adult autistics and multi-handicapped patients in a public mental health unit. [Les traitements psychotropes prescrits dans un etablissement public de sante mentale accueillant des personnes atteintes d'autisme et des polyhandicapes] L'Encephale, 35(4), 370-376. doi:10.1016/j.encep.2008.04.008 [doi]

Autism Speaks. (2015). 10 year timeline. Retrieved from https://www.autismspeaks.org/10year/timeline

Baribeau, D. A., \& Anagnostou, E. (2014). An update on medication management of behavioral disorders in autism. Current Psychiatry Reports, 16(3), 437-014-0437-0. doi:10.1007/s11920-014-0437-0 [doi]

Barnard-Brak, L., Davis, T. N., Schmidt, M., \& Richman, D. M. (2014). Effects associated with on- and off-label stimulant treatment of core autism and ADHD symptoms exhibited by children with autism spectrum disorder. Developmental Neurorehabilitation, doi:10.3109/17518423.2014.904949 [doi]

Blankenship, K., Erickson, C. A., Stigler, K. A., Posey, D. J., \& McDougle, C. J. (2010). Aripiprazole for irritability associated with autistic disorder in children and adolescents aged 6-17 years. Pediatric Health, 4(4), 375-381.

Bruder, M. B., Kerins, G., Mazzarella, C., Sims, J., \& Stein, N. (2012). Brief report: The medical care of adults with autism spectrum disorders: Identifying the needs. Journal of Autism and Developmental Disorders, 42(11), 2498-2504. doi:10.1007/s10803-012-1496-x [doi]

Buck, T. R., Viskochil, J., Farley, M., Coon, H., McMahon, W. M., Morgan, J., \& Bilder, D. A. (2014a). Psychiatric comorbidity and medication use in adults with autism spectrum disorder. Journal of Autism and Developmental Disorders, doi:10.1007/s10803-014-2170-2 [doi]

Buck, T. R., Viskochil, J., Farley, M., Coon, H., McMahon, W. M., Morgan, J., \& Bilder, D. A. (2014b). Psychiatric comorbidity and medication use in adults with autism spectrum disorder. Journal of Autism and Developmental Disorders, doi:10.1007/s10803-014-2170-2 [doi]

Buie, T., Campbell, D. B., Fuchs, G. J.,3rd, Furuta, G. T., Levy, J., Vandewater, J., . . Winter, H. (2010). Evaluation, diagnosis, and treatment of gastrointestinal disorders in individuals with ASDs: A consensus report. Pediatrics, 125 Suppl 1, S1-18. doi:10.1542/peds.2009-1878C [doi]

Ching, H., \& Pringsheim, T. (2012). Aripiprazole for autism spectrum disorders (ASD). The Cochrane Database of Systematic Reviews, 5, CD009043. doi:10.1002/14651858.CD009043.pub2; 10.1002/14651858.CD009043.pub2

Coury, D. L., Anagnostou, E., Manning-Courtney, P., Reynolds, A., Cole, L., McCoy, R., . . Perrin, J. M. (2012a). Use of psychotropic medication in children and adolescents with autism spectrum disorders. Pediatrics, 130 Suppl 2, S69-76. doi:10.1542/peds.2012-0900D; 10.1542/peds.2012-0900D 


\section{PhD Dissertation Chapter 4}

Coury, D. L., Anagnostou, E., Manning-Courtney, P., Reynolds, A., Cole, L., McCoy, R., . . Perrin, J. M. (2012b). Use of psychotropic medication in children and adolescents with autism spectrum disorders. Pediatrics, 130 Suppl 2, S69-76. doi:10.1542/peds.2012-0900D; 10.1542/peds.2012-0900D

Croen, L. A., Najjar, D. V., Ray, G. T., Lotspeich, L., \& Bernal, P. (2006). A comparison of health care utilization and costs of children with and without autism spectrum disorders in a large group-model health plan. Pediatrics, 118(4), e1203-11. doi:118/4/e1203 [pii]

Croen, L. A., Zerbo, O., Qian, Y., Massolo, M. L., Rich, S., Sidney, S., \& Kripke, C. (2015). The health status of adults on the autism spectrum. Autism: The International Journal of Research and Practice, doi:1362361315577517 [pii]

Curran, M. P. (2011). Aripiprazole in the treatment of irritability associated with autistic disorder in paediatric patients: Profile report. CNS Drugs, 25(9), 801-802. doi:10.2165/11208280-000000000-00000 [doi]

Dalsgaard, S., Nielsen, H. S., \& Simonsen, M. (2013). Five-fold increase in national prevalence rates of attentiondeficit/hyperactivity disorder medications for children and adolescents with autism spectrum disorder, attention-deficit/hyperactivity disorder, and other psychiatric disorders: A Danish register-based study. Journal of Child and Adolescent Psychopharmacology, 23(7), 432-439. doi:10.1089/cap.2012.0111 [doi]

Dove, D., Warren, Z., McPheeters, M. L., Taylor, J. L., Sathe, N. A., \& Veenstra-VanderWeele, J. (2012). Medications for adolescents and young adults with autism spectrum disorders: A systematic review. Pediatrics, 130(4), 717-726. doi:10.1542/peds.2012-0683; 10.1542/peds.2012-0683

Esbensen, A. J., Greenberg, J. S., Seltzer, M. M., \& Aman, M. G. (2009). A longitudinal investigation of psychotropic and non-psychotropic medication use among adolescents and adults with autism spectrum disorders. Journal of Autism and Developmental Disorders, 39(9), 1339-1349. doi:10.1007/s10803-009-07503 [doi]

Frazier, T. W., Shattuck, P. T., Narendorf, S. C., Cooper, B. P., Wagner, M., \& Spitznagel, E. L. (2011a). Prevalence and correlates of psychotropic medication use in adolescents with an autism spectrum disorder with and without caregiver-reported attention-deficit/hyperactivity disorder. Journal of Child and Adolescent Psychopharmacology, 21(6), 571-579. doi:10.1089/cap.2011.0057; 10.1089/cap.2011.0057

Frazier, T. W., Shattuck, P. T., Narendorf, S. C., Cooper, B. P., Wagner, M., \& Spitznagel, E. L. (2011b). Prevalence and correlates of psychotropic medication use in adolescents with an autism spectrum disorder with and without caregiver-reported attention-deficit/hyperactivity disorder. Journal of Child and Adolescent Psychopharmacology, 21(6), 571-579. doi:10.1089/cap.2011.0057 [doi]

Gerhard, T., Chavez, B., Olfson, M., \& Crystal, S. (2009). National patterns in the outpatient pharmacological management of children and adolescents with autism spectrum disorder. Journal of Clinical Psychopharmacology, 29(3), 307-310. doi:10.1097/JCP.0b013e3181a20c8a; 10.1097/JCP.0b013e3181a20c8a

Golnik, A., Ireland, M., \& Borowsky, I. W. (2009). Medical homes for children with autism: A physician survey. Pediatrics, 123(3), 966-971. doi:10.1542/peds.2008-1321 [doi]

Heidgerken, A. D., Geffken, G., Modi, A., \& Frakey, L. (2005). A survey of autism knowledge in a health care setting. Journal of Autism and Developmental Disorders, 35(3), 323-330.

Hirota, T., Veenstra-Vanderweele, J., Hollander, E., \& Kishi, T. (2014). Antiepileptic medications in autism spectrum disorder: A systematic review and meta-analysis. Journal of Autism and Developmental Disorders, 44(4), 948-957. doi:10.1007/s10803-013-1952-2 [doi] 


\section{PhD Dissertation Chapter 4}

Hollander, E., Wang, A. T., Braun, A., \& Marsh, L. (2009). Neurological considerations: Autism and parkinson's disease. Psychiatry Research, 170(1), 43-51. doi:10.1016/j.psychres.2008.07.014 [doi]

Jang, J., Dixon, D. R., Tarbox, J., \& Granpeesheh, D. (2011). Symptom severity and challenging behavior in children with ASD. Research in Autism Spectrum Disorders, 5(3), 1028-1032.

Kato, K., Mikami, K., Akama, F., Yamada, K., Maehara, M., Kimoto, K., . . Matsumoto, H. (2013). Clinical features of suicide attempts in adults with autism spectrum disorders. General Hospital Psychiatry, 35(1), 5053. doi:10.1016/j.genhosppsych.2012.09.006 [doi]

Khanna, R., Jariwala, K., \& West-Strum, D. (2013). Use and cost of psychotropic drugs among recipients with autism in a state Medicaid fee-for-service program. Journal of Intellectual Disability Research: JIDR, 57(2), 161-171. doi:10.1111/j.1365-2788.2012.01563.x; 10.1111/j.1365-2788.2012.01563.x

Kohane, I. S., McMurry, A., Weber, G., MacFadden, D., Rappaport, L., Kunkel, L., . . . Churchill, S. (2012). The co-morbidity burden of children and young adults with autism spectrum disorders. PloS One, 7(4), e33224. doi:10.1371/journal.pone.0033224; 10.1371/journal.pone.0033224

Lake, J. K., Balogh, R., \& Lunsky, Y. (2012). Polypharmacy profiles and predictors among adults with autism spectrum disorders. Research in Autism Spectrum Disorders, 6(3), 1142-1149.

LeClerc, S., \& Easley, D. (2015). Pharmacological therapies for autism spectrum disorder: A review. P \& T: A PeerReviewed Journal for Formulary Management, 40(6), 389-397.

Logan, S. L., Nicholas, J. S., Carpenter, L. A., King, L. B., Garrett-Mayer, E., \& Charles, J. M. (2012). High prescription drug use and associated costs among Medicaid-eligible children with autism spectrum disorders identified by a population-based surveillance network. Annals of Epidemiology, 22(1), 1-8. doi:10.1016/j.annepidem.2011.10.007; 10.1016/j.annepidem.2011.10.007

Mandell, D. S., Morales, K. H., Marcus, S. C., Stahmer, A. C., Doshi, J., \& Polsky, D. E. (2008a). Psychotropic medication use among Medicaid-enrolled children with autism spectrum disorders. Pediatrics, 121(3), e441-8. doi:10.1542/peds.2007-0984; 10.1542/peds.2007-0984

Mandell, D. S., Morales, K. H., Marcus, S. C., Stahmer, A. C., Doshi, J., \& Polsky, D. E. (2008b). Psychotropic medication use among Medicaid-enrolled children with autism spectrum disorders. Pediatrics, 121(3), e441-8. doi:10.1542/peds.2007-0984; 10.1542/peds.2007-0984

Marcus, R. N., Owen, R., Kamen, L., Manos, G., McQuade, R. D., Carson, W. H., \& Aman, M. G. (2009). A placebo-controlled, fixed-dose study of aripiprazole in children and adolescents with irritability associated with autistic disorder. Journal of the American Academy of Child and Adolescent Psychiatry, 48(11), 11101119. doi:10.1097/CHI.0b013e3181b76658 [doi]

Matson, J. L., \& Rivet, T. T. (2008). Characteristics of challenging behaviors in adults with autistic disorder, PDDNOS, and intellectual disability. Journal of Intellectual \& Developmental Disability, 33(4), 323-329. doi:10.1080/13668250802492600; 10.1080/13668250802492600

Matson, J. L., Sipes, M., Fodstad, J. C., \& Fitzgerald, M. E. (2011). Issues in the management of challenging behaviors of adults with autism spectrum disorder. CNS Drugs, 25(7), 597-606. doi:10.2165/11591700000000000-00000 [doi]

Matson, J. L., Wilkins, J., \& Macken, J. (2009). The relationship of challenging behaviors to severity and symptoms of autism spectrum disorders. Journal of Mental Health Research in Intellectual Disabilities, 2(1), 29-44.

Mauch, D., Pfefferle, S., Booker, C., Pustell, M., \& Levin, J. (2011). Report on state services to individuals 


\section{PhD Dissertation Chapter 4}

with autism spectrum disorders (ASD). ( No. S-10 CMS-33 No. 2). Centers for Medicare \& Medicaid Services (CMS) ASD Services Project: Centers for Medicare \& Medicaid Services (CMS).

McCracken, J. T., McGough, J., Shah, B., Cronin, P., Hong, D., Aman, M. G., . . Research Units on Pediatric Psychopharmacology Autism Network. (2002). Risperidone in children with autism and serious behavioral problems. The New England Journal of Medicine, 347(5), 314-321. doi:10.1056/NEJMoa013171

McElhanon, B. O., McCracken, C., Karpen, S., \& Sharp, W. G. (2014). Gastrointestinal symptoms in autism spectrum disorder: A meta-analysis. Pediatrics, doi: peds.2013-3995 [pii]

Murphy, G. H., Beadle-Brown, J., Wing, L., Gould, J., Shah, A., \& Holmes, N. (2005). Chronicity of challenging behaviours in people with severe intellectual disabilities and/or autism: A total population sample. Journal of Autism \& Developmental Disorders, 35(4), 405-418. doi:10.1007/s10803-005-5030-2

Myer, P. A., Mannalithara, A., Singh, G., Singh, G., Pasricha, P. J., \& Ladabaum, U. (2013). Clinical and economic burden of emergency department visits due to gastrointestinal diseases in the United States. The American Journal of Gastroenterology, 108(9), 1496-1507. doi:10.1038/ajg.2013.199 [doi]

Myers, S. M. (2007). The status of pharmacotherapy for autism spectrum disorders. Expert Opinion on Pharmacotherapy, 8(11), 1579-1603. doi:10.1517/14656566.8.11.1579 [doi]

National Committee for Quality Assurance. (2015). HEDIS and quality measurement. HEDIS 2015 final NDC lists.

National Conference of State Legislatures. (2014). Recent Medicaid prescription drug laws and strategies. Retrieved from http://www.ncsl.org/research/health/medicaid-pharmaceutical-laws-and-policies.aspx

Oskoui, M., \& Wolfson, C. (2012). Treatment comfort of adult neurologists in childhood onset conditions. The Canadian Journal of Neurological Sciences.Le Journal Canadien Des Sciences Neurologiques, 39(2), 202205. doi:HM223822024126U6 [pii]

Oswald, D. P., \& Sonenklar, N. A. (2007a). Medication use among children with autism spectrum disorders. Journal of Child and Adolescent Psychopharmacology, 17(3), 348-355. doi:10.1089/cap.2006.17303

Oswald, D. P., \& Sonenklar, N. A. (2007b). Medication use among children with autism spectrum disorders. Journal of Child and Adolescent Psychopharmacology, 17(3), 348-355. doi:10.1089/cap.2006.17303 [doi]

Owen, R., Sikich, L., Marcus, R. N., Corey-Lisle, P., Manos, G., McQuade, R. D., . . Findling, R. L. (2009). Aripiprazole in the treatment of irritability in children and adolescents with autistic disorder. Pediatrics, 124(6), 1533-1540. doi:10.1542/peds.2008-3782 [doi]

Quality Resource Systems, I. (2006). Area resource file. (). Fairfax, VA: Health Resources and Services Administration, US Department of Health and Human Services, 2006.

Rosenberg, R. E., Mandell, D. S., Farmer, J. E., Law, J. K., Marvin, A. R., \& Law, P. A. (2010a). Psychotropic medication use among children with autism spectrum disorders enrolled in a national registry, 2007-2008. Journal of Autism and Developmental Disorders, 40(3), 342-351. doi:10.1007/s10803-009-0878-1; 10.1007/s10803-009-0878-1

Rosenberg, R. E., Mandell, D. S., Farmer, J. E., Law, J. K., Marvin, A. R., \& Law, P. A. (2010b). Psychotropic medication use among children with autism spectrum disorders enrolled in a national registry, 2007-2008. Journal of Autism and Developmental Disorders, 40(3), 342-351. doi:10.1007/s10803-009-0878-1; 10.1007/s10803-009-0878-1 


\section{PhD Dissertation Chapter 4}

Rubenstein, E., Wiggins, L. D., \& Lee, L. C. (2015). A review of the differences in developmental, psychiatric, and medical endophenotypes between males and females with autism spectrum disorder. Journal of Developmental and Physical Disabilities, 27(1), 119-139. doi:10.1007/s10882-014-9397-x [doi]

Ruble, L. A., Heflinger, C. A., Renfrew, J. W., \& Saunders, R. C. (2005). Access and service use by children with autism spectrum disorders in Medicaid managed care. Journal of Autism and Developmental Disorders, 35(1), $3-13$.

Schubart, J. R., Camacho, F., \& Leslie, D. (2014). Psychotropic medication trends among children and adolescents with autism spectrum disorder in the medicaid program. Autism: The International Journal of Research and Practice, 18(6), 631-637. doi:10.1177/1362361313497537 [doi]

Semansky, R. M., Xie, M., \& Mandell, D. S. (2011). Medicaid's increasing role in treating youths with autism spectrum disorders. Psychiatric Services (Washington, D.C.), 62(6), 588-588.

Simonoff, E., Pickles, A., Charman, T., Chandler, S., Loucas, T., \& Baird, G. (2008). Psychiatric disorders in children with autism spectrum disorders: Prevalence, comorbidity, and associated factors in a populationderived sample. Journal of the American Academy of Child and Adolescent Psychiatry, 47(8), 921-929. doi:10.1097/CHI.0b013e318179964f [doi]

Spencer, D., Marshall, J., Post, B., Kulakodlu, M., Newschaffer, C., Dennen, T., . . Jain, A. (2013). Psychotropic medication use and polypharmacy in children with autism spectrum disorders. Pediatrics, 132(5), 833-840. doi:10.1542/peds.2012-3774; 10.1542/peds.2012-3774

Starkstein, S., Gellar, S., Parlier, M., Payne, L., \& Piven, J. (2015). High rates of Parkinsonism in adults with autism. Journal of Neurodevelopmental Disorders, 7(1), 29-015-9125-6. Epub 2015 Aug 30. doi:10.1186/s11689-015-9125-6 [doi]

The Henry J Kaiser Family Foundation. (2013). State HMO penetration rate, state health facts. Retrieved from http://kff.org/other/state-indicator/hmo-penetration-rate/

van Steensel, F. J., Bogels, S. M., \& de Bruin, E. I. (2013). Psychiatric comorbidity in children with autism spectrum disorders: A comparison with children with ADHD. Journal of Child and Family Studies, 22(3), 368-376. doi:10.1007/s10826-012-9587-z [doi]

Volkmar, F., Cook, E. H.,Jr, Pomeroy, J., Realmuto, G., \& Tanguay, P. (1999). Practice parameters for the assessment and treatment of children, adolescents, and adults with autism and other pervasive developmental disorders. american academy of child and adolescent psychiatry working group on quality issues. Journal of the American Academy of Child and Adolescent Psychiatry, 38(12 Suppl), 32S-54S.

Warren, Z., Veenstra-VanderWeele, J., Stone, W., Bruzek, J. L., Nahmias, A. S., Foss-Feig, J. H., . . McPheeters, M. L. (April 2011). Therapies for children with autism spectrum disorders. (Comparative Effectiveness Reviews no. 26 No. 11-EHC029-EF). Rockville (MD): Agency for Healthcare Research and Quality. doi:http://effectivehealthcare.ahrq.gov/ehc/products/106/651/Autism_Disorder_exec-summ.pdf

West, L., Brunssen, S. H., \& Waldrop, J. (2009). Review of the evidence for treatment of children with autism with selective serotonin reuptake inhibitors. Journal for Specialists in Pediatric Nursing: JSPN, 14(3), 183-191. doi:10.1111/j.1744-6155.2009.00196.x [doi]

Williams, K., Wheeler, D. M., Silove, N., \& Hazell, P. (2010). Selective serotonin reuptake inhibitors (SSRIs) for autism spectrum disorders (ASD). The Cochrane Database of Systematic Reviews, (8):CD004677. doi(8), CD004677. doi:10.1002/14651858.CD004677.pub2; 10.1002/14651858.CD004677.pub2 


\section{PhD Dissertation Chapter 4}

Zito, J. M., Derivan, A. T., Kratochvil, C. J., Safer, D. J., Fegert, J. M., \& Greenhill, L. L. (2008). Off-label psychopharmacologic prescribing for children: History supports close clinical monitoring. Child and Adolescent Psychiatry and Mental Health, 2(1), 24. doi:10.1186/1753-2000-2-24 [doi] 
PhD Dissertation Chapter 4

Tables

Table 1. Description of type of prescription drugs and polypharmacy by ASD status

Adults with and without ASD matched on age, race, and gender $(\mathbf{n}=7,092)$

Medicaid Analytic Extract 2000-2008 (IL,NY, and TX)

\begin{tabular}{|c|c|c|c|c|c|}
\hline \multirow[b]{2}{*}{ Rx type } & \multicolumn{2}{|c|}{ ASD } & \multicolumn{2}{|c|}{ No ASD } & \multirow[b]{2}{*}{ Sig. } \\
\hline & $\mathbf{N}$ & $\mathrm{Col} \%$ & $\mathbf{N}$ & Col \% & \\
\hline & 1,772 & 100.0 & 5,320 & 100.0 & \\
\hline Any prescription drug & & & & & *** \\
\hline$>25 \mathrm{Rx}$ claims & 1,230 & 69.4 & 1,486 & 27.9 & \\
\hline$<=25 \mathrm{Rx}$ claims & 448 & 25.3 & 3,137 & 59.0 & \\
\hline no Rx claim & 94 & 5.3 & 697 & 13.1 & \\
\hline Dermatologic agents & 850 & 48.0 & 1,711 & 32.2 & $* * *$ \\
\hline Respiratory agents & 679 & 38.3 & 1,821 & 34.2 & $* * *$ \\
\hline Gastrointestinal agents & 560 & 31.6 & 1,235 & 23.2 & $* * *$ \\
\hline Alternative medicine/nutritional supplements & 437 & 24.7 & 964 & 18.1 & $* * *$ \\
\hline Cardiovascular agents & 411 & 23.2 & 1,026 & 19.3 & $* * *$ \\
\hline Antiparkinsonian agents & 401 & 22.6 & 523 & 9.8 & $* * *$ \\
\hline Statins/other antihyperlipidemics & 129 & 7.3 & 266 & 5.0 & $* * *$ \\
\hline Immunologics & 35 & 2.0 & 37 & 0.7 & $* * *$ \\
\hline Other metabolic agents & 25 & 1.4 & 57 & 1.1 & $* * *$ \\
\hline Antimicrobials & 822 & 46.4 & 2,913 & 54.8 & $* * *$ \\
\hline Analgesics & 503 & 28.4 & 2,615 & 49.2 & $* * *$ \\
\hline Hormone modifiers/steroids & 281 & 15.9 & 909 & 17.1 & $* * *$ \\
\hline CNS agents (excluding stimulants) & 74 & 4.2 & 540 & 10.2 & $* * *$ \\
\hline Antidiabetics & 62 & 3.5 & 262 & 4.9 & $* * *$ \\
\hline Blood modifiers & 44 & 2.5 & 219 & 4.1 & $* * *$ \\
\hline Anticancer ${ }^{\S}$ & 13 & 0.7 & 121 & 2.3 & \\
\hline Psychotropic medications & 1,510 & 85.2 & 2,254 & 42.4 & **** \\
\hline Antipsychotics & 1,170 & 66.0 & 1,072 & 20.2 & **** \\
\hline Anticonvulsants & 1,048 & 59.1 & 1,042 & 19.6 & $* * *$ \\
\hline Antidepressants & 652 & 36.8 & 1,361 & 25.6 & $* * *$ \\
\hline Anxiolytics/Hypnotics/Sedatives & 380 & 21.4 & 587 & 11.0 & $* * *$ \\
\hline CNS stimulants & 33 & 1.9 & 54 & 1.0 & $* * *$ \\
\hline \multicolumn{6}{|l|}{ Polypharmacy } \\
\hline General polypharmacy ( $>=6$ classes $)$ & 856 & 48.3 & 1,727 & 32.5 & *** \\
\hline Psychotropic polypharmacy ( $>=3$ classes) & 337 & 19.0 & 312 & 5.9 & $* * *$ \\
\hline ASD vs. no ASD & OR & $5 \% \mathrm{CI})$ & AOR & $95 \% \mathrm{CI})$ & \\
\hline$>=6$ classes vs. $<6$ classes & $1.74 * * *$ & $(1.56,1.95)$ & $1.17^{*}$ & $(1.03,1.33)$ & \\
\hline$>=3$ vs. $<3$ psychotropic classes & $1.65^{* * * *}$ & $(1.39,1.96)$ & $1.40 * * *$ & $(1.17,1.68)$ & \\
\hline
\end{tabular}

Note: Based on chi-square estimates for fee for service enrolled individuals aged 22-64 years with no Medicare coverage and alive in IL, NY, and TX Medicaid from 2000-2008. ASD: Autism Spectrum Disorders; Rx:

Prescription Drug; Col \%: Column percentages; Fischer exact tests were conducted for variables with expected cell count $<5$. ${ }^{\S}$ Chi-square test was not conducted due to very low cell sizes. OR: Odds ratio; represents estimates from 


\section{PhD Dissertation Chapter 4}

multinomial logistic regression analyses using ASD status. AOR: Adjusted odds ratio; represents estimates from multinomial logistic regression analyses respectively after adjusting for cohort (before/after 2004), state, eligibility, county characteristics (metro status, median household income, above high school education, PCP shortage area, mental health specialist shortage area, and psychiatrist density), and number of comorbidities. Sig.: *** $\mathrm{P}<0.001$; $* * 0.001 \leq \mathrm{P}<0.01 ; * 0.01 \leq \mathrm{P}<0.05$. 
Table 2a. Mean number of claims for specific Rx classes per year Adults with and without ASD matched on age, race, and gender $(\mathbf{n}=7,092)$ Medicaid Analytic Extract 2000-2008 (IL,NY, and TX)

\begin{tabular}{|c|c|c|c|c|}
\hline & ASD & No ASD & & \\
\hline & Mean (SD) & Mean (SD) & $\begin{array}{l}\text { Ratio of Means } \\
\text { ASD vs. No ASD }\end{array}$ & Sig. \\
\hline Any prescription drug & $48.15(37)$ & $21.36(29)$ & 2.25 & $* * *$ \\
\hline Respiratory agents & $2.50(6)$ & $1.85(6)$ & 1.35 & $* *$ \\
\hline Antimicrobials & $1.34(3)$ & $2.79(8)$ & 0.48 & $* * *$ \\
\hline Anticancer & $0.07(2)$ & $0.03(1)$ & 0.00 & \\
\hline Immunologics & $0.02(0)$ & $0.03(1)$ & 0.00 & \\
\hline Cardiovascular agents & $2.77(7)$ & $1.77(6)$ & 1.56 & $* * *$ \\
\hline Analgesics & $0.85(2)$ & $2.11(5)$ & 0.40 & $* * *$ \\
\hline Blood modifiers & $0.20(1)$ & $0.23(2)$ & 0.00 & \\
\hline Hormone modifiers/steroids & $1.44(4)$ & $0.78(3)$ & 1.85 & $* * *$ \\
\hline Statins/other antihyperlipidemics & $0.74(3)$ & $0.27(2)$ & 2.74 & $* * *$ \\
\hline Antidiabetics & $0.40(3)$ & $0.50(3)$ & 0.80 & \\
\hline Dermatologic agents & $2.83(6)$ & $1.16(3)$ & 2.44 & $* * *$ \\
\hline $\begin{array}{l}\text { Alternative medicine/nutritional } \\
\text { supplements }\end{array}$ & $2.38(6)$ & $0.87(3)$ & 2.74 & $* * *$ \\
\hline Gastrointestinal agents & $3.52(8)$ & $1.16(4)$ & 3.03 & $* * *$ \\
\hline CNS agents (excluding stimulants) & $0.37(2)$ & $0.35(2)$ & 1.00 & \\
\hline Antiparkinsonian agents & $1.86(4)$ & $0.48(2)$ & 3.88 & $* * *$ \\
\hline Other metabolic agents & $0.15(1)$ & $0.05(1)$ & 3.00 & $* * *$ \\
\hline Psychotropic medications & $25.51(21)$ & $6.26(12)$ & 4.08 & $* * *$ \\
\hline Antidepressants & $3.93(6)$ & $1.79(4)$ & 2.20 & $* * *$ \\
\hline Antipsychotics & $9.90(10)$ & $2.04(6)$ & 4.85 & $* * *$ \\
\hline Anticonvulsants & $10.28(14)$ & $1.84(6)$ & 5.59 & $* * *$ \\
\hline CNS stimulants & $0.14(1)$ & $0.05(1)$ & 2.80 & $* * *$ \\
\hline Anxiolytics/Hypnotics/Sedatives & $1.25(3)$ & $0.54(2)$ & 2.31 & $* * *$ \\
\hline
\end{tabular}

Based on t-test estimates for fee for service enrolled individuals aged 22-64 years with no Medicare coverage and alive in IL, NY, and TX Medicaid from 2000-2008. Sig.: ***P $<0.001 ; * * 0.001 \leq \mathrm{P}<$ $0.01 ; * 0.01 \leq \mathrm{P}<0.05$. 
Table 2b. Mean number of claims for specific Rx classes per year for users $\epsilon$ Adults with and without ASD matched on age ,race, and gender $(n=7,092)$

Medicaid Analytic Extract 2000-2008 (IL,NY, and TX)

\begin{tabular}{|c|c|c|c|c|}
\hline & ASD & No ASD & & \\
\hline & Mean (SD) & Mean (SD) & $\begin{array}{l}\text { Ratio of Means ASD } \\
\text { vs. No ASD }\end{array}$ & Sig. \\
\hline Any prescription drug & $50(36)$ & $25(30)$ & 2.00 & $* * *$ \\
\hline Respiratory agents & $7(8)$ & $5(8)$ & 1.40 & $* *$ \\
\hline Antimicrobials & $3(3)$ & $5(10)$ & 0.60 & $* * *$ \\
\hline Anticancer & $10(17)$ & $6(7)$ & 1.66 & \\
\hline Immunologics & $1(0)$ & $4(6)$ & 0.25 & $* * *$ \\
\hline Cardiovascular agents & $12(9)$ & $9(10)$ & 1.33 & $* * *$ \\
\hline Analgesics & $3(3)$ & $4(6)$ & 0.75 & $* * *$ \\
\hline Blood modifiers & $8(6)$ & $6(6)$ & 1.33 & \\
\hline Hormone modifiers/steroids & $9(6)$ & $5(5)$ & 1.80 & $* * *$ \\
\hline Statins/other antihyperlipidemics & $10(5)$ & $5(4)$ & 2.00 & $* * *$ \\
\hline Antidiabetics & $12(8)$ & $10(9)$ & 1.20 & \\
\hline Dermatologic agents & $6(7)$ & $4(5)$ & 1.50 & $* * *$ \\
\hline $\begin{array}{l}\text { Alternative medicine/nutritional } \\
\text { supplements }\end{array}$ & $10(8)$ & $5(6)$ & 2.00 & $* * *$ \\
\hline Gastrointestinal agents & $11(10)$ & $5(6)$ & 2.20 & $* * *$ \\
\hline CNS agents (excluding stimulants) & $9(7)$ & $3(4)$ & 3.00 & $* * *$ \\
\hline Antiparkinsonian agents & $8(6)$ & $5(5)$ & 1.60 & $* * *$ \\
\hline Other metabolic agents & $10(5)$ & $5(4)$ & 2.00 & $* * *$ \\
\hline Psychotropic medications & $30(20)$ & $15(16)$ & 2.00 & $* * *$ \\
\hline Antidepressants & $11(6)$ & $7(6)$ & 1.57 & $* * *$ \\
\hline Antipsychotics & $15(9)$ & $10(10)$ & 1.50 & $* * *$ \\
\hline Anticonvulsants & $17(14)$ & $9(9)$ & 1.88 & $* * *$ \\
\hline CNS stimulants & $7(6)$ & $5(4)$ & 1.40 & $* *$ \\
\hline Anxiolytics/Hypnotics/Sedatives & $6(5)$ & $5(5)$ & 1.20 & $* *$ \\
\hline
\end{tabular}

Based on t-test estimates for fee for service enrolled individuals aged 22-64 years with no Medicare coverage and alive in IL, NY, and TX Medicaid from 2000-2008.

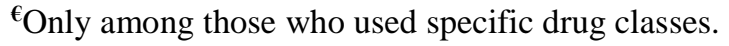

Sig.: ***P $<0.001 ; * * 0.001 \leq \mathrm{P}<0.01 ; * 0.01 \leq \mathrm{P}<0.05$. 
PhD Dissertation Chapter 4

Table 3. Description of Sample by General and Psychotropic Polypharmacy Among Adults with ASD $(n=1,772)$

Medicaid Analytic Extract 2000-2008 (IL,NY, and TX)

\begin{tabular}{|c|c|c|c|c|c|c|c|}
\hline & & \multicolumn{2}{|c|}{$\begin{array}{c}\text { General } \\
\text { Polypharmacy } \\
\text { (6 or more) } \\
\end{array}$} & \multicolumn{4}{|c|}{$\begin{array}{c}\text { Psychotropic } \\
\begin{array}{c}\text { Polypharmacy ( } 3 \text { or } \\
\text { more) }\end{array} \\
\end{array}$} \\
\hline & & $\mathbf{N}$ & Row\% & Sig. & $\mathbf{N}$ & Row\% & \\
\hline ALL & & 856 & $48 \%$ & $* * *$ & 337 & $19 \%$ & Sig. \\
\hline \multirow[t]{9}{*}{ Identification year } & & & & $* * *$ & & & \\
\hline & 2000 & 260 & 42.1 & & 110 & 17.8 & \\
\hline & 2001 & 113 & 45.2 & & 48 & 19.2 & \\
\hline & 2002 & 82 & 44.3 & & 31 & 16.8 & \\
\hline & 2003 & 81 & 50.0 & & 27 & 16.7 & \\
\hline & 2004 & 86 & 52.8 & & 27 & 16.6 & \\
\hline & 2005 & 68 & 52.7 & & 30 & 23.3 & \\
\hline & 2006 & 54 & 58.7 & & 24 & 26.1 & \\
\hline & 2007 & 112 & 64.4 & & 40 & 23 & \\
\hline \multirow[t]{3}{*}{ Sex } & & & & $* * *$ & & & \\
\hline & female & 281 & 55.5 & & 101 & 20 & \\
\hline & male & 575 & 45.4 & & 236 & 18.6 & \\
\hline \multirow[t]{3}{*}{ Age } & & & & $* * *$ & & & \\
\hline & $22-40$ & 700 & 46.1 & & 282 & 18.6 & \\
\hline & $41-64$ & 156 & 61.2 & & 55 & 21.6 & \\
\hline \multirow[t]{4}{*}{ Race } & & & & $* * *$ & & & $* * *$ \\
\hline & White & 358 & 54.4 & & 166 & 25.2 & \\
\hline & African American & 172 & 47.3 & & 50 & 13.7 & \\
\hline & Other & 326 & 43.5 & & 121 & 16.1 & \\
\hline \multirow[t]{6}{*}{ Eligibility } & & & & $* *$ & & & $* *$ \\
\hline & Cash & 681 & 46.8 & & 259 & 17.8 & \\
\hline & No cash & 175 & 55.4 & & 78 & 24.7 & \\
\hline & & & & $* *$ & & & \\
\hline & Medically needy & 50 & 64.9 & & 19 & 24.7 & \\
\hline & $\begin{array}{l}\text { Not medically } \\
\text { needy }\end{array}$ & 806 & 47.6 & & 318 & 18.8 & \\
\hline \multicolumn{8}{|l|}{$\begin{array}{l}\text { County } \\
\text { Characteristics }\end{array}$} \\
\hline \multicolumn{8}{|l|}{ Metro } \\
\hline & yes & 686 & 47.3 & & 264 & 18.2 & \\
\hline & no & 170 & 52.8 & & 73 & 22.7 & \\
\hline \multicolumn{8}{|l|}{ PCP shortage area } \\
\hline & yes & 753 & 47.8 & & 304 & 19.3 & \\
\hline & no & 103 & 52.3 & & 33 & 16.8 & \\
\hline \multicolumn{8}{|c|}{ Mental health specialist shortage area } \\
\hline & yes & 682 & 47.6 & & 268 & 18.7 & \\
\hline
\end{tabular}


PhD Dissertation Chapter 4

Table 3. Description of Sample by General and Psychotropic Polypharmacy Among Adults with ASD $(n=1,772)$

Medicaid Analytic Extract 2000-2008 (IL,NY, and TX)

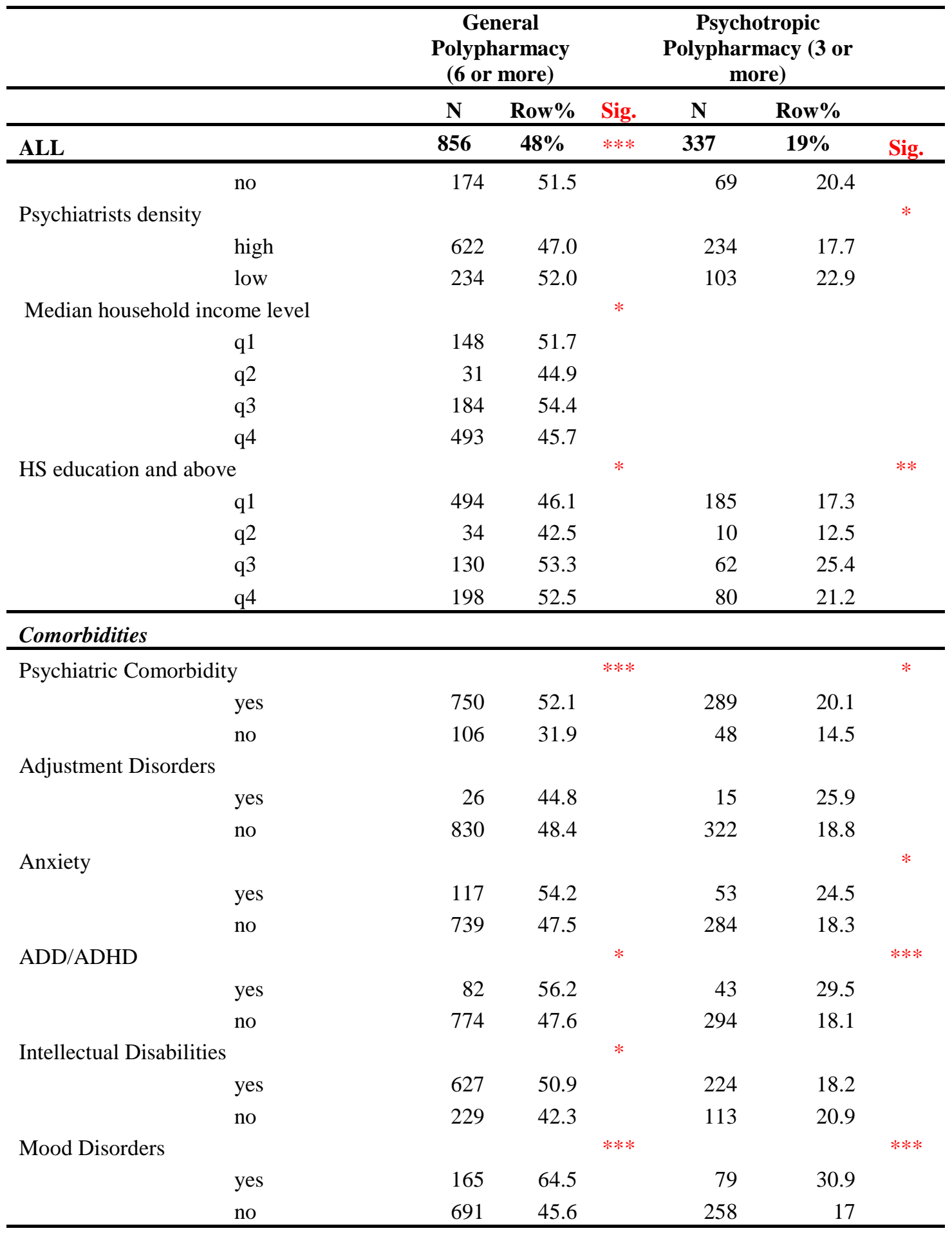


PhD Dissertation Chapter 4

Table 3. Description of Sample by General and Psychotropic Polypharmacy Among Adults with ASD $(\mathbf{n}=1,772)$

Medicaid Analytic Extract 2000-2008 (IL,NY, and TX)

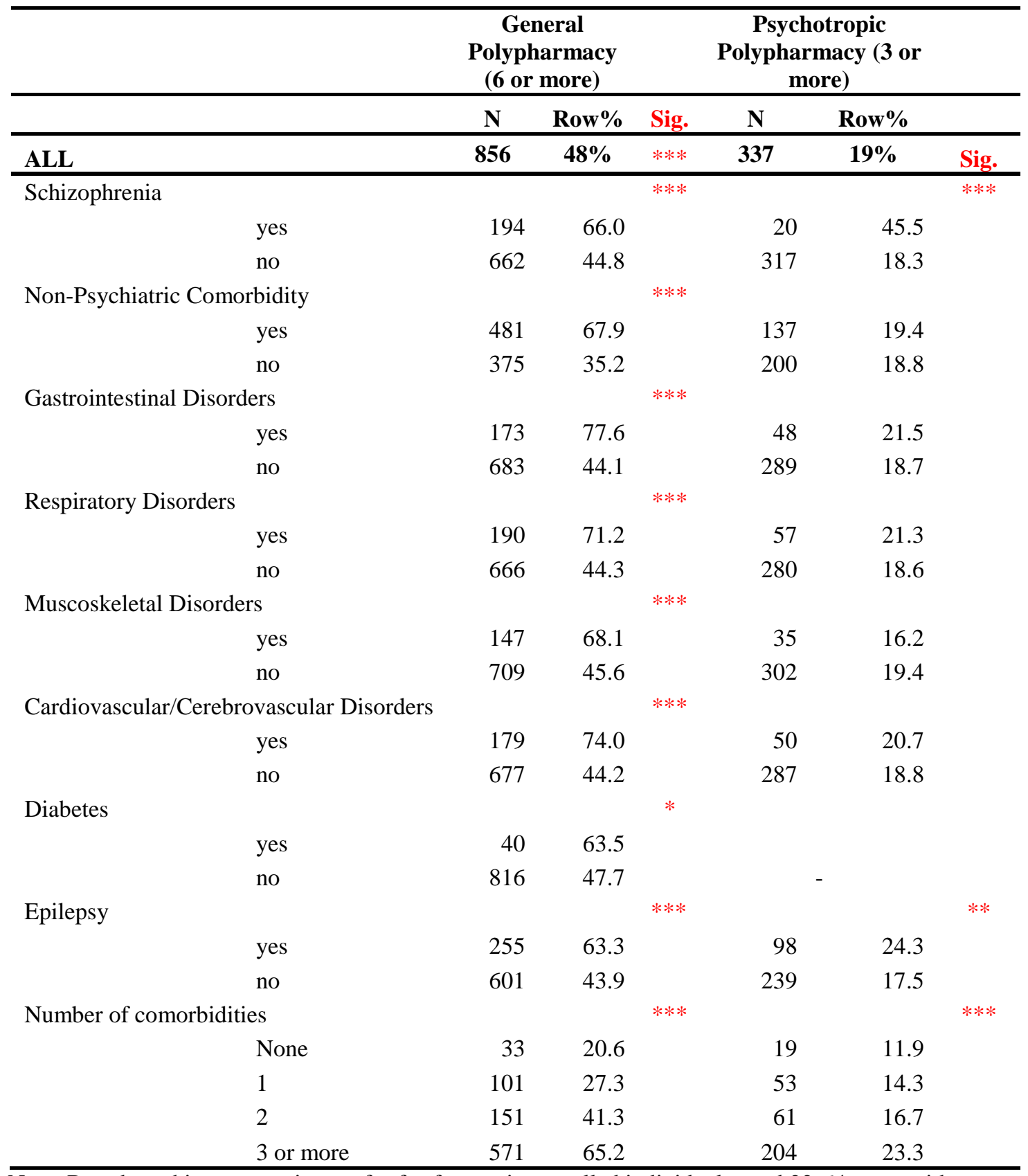

Note: Based on chi-square estimates for fee for service enrolled individuals aged 22-64 years with no Medicare coverage and alive in IL, NY, and TX Medicaid from 2000-2008.ASD: Autism Spectrum Disorders; Rx: Prescription Drug; PCP: Primary Care Provider; HS: High School. Fischer exact tests were conducted for variables with expected cell count $<5$.

Chi-square tests were not conducted and findings are not presented for cell sizes $\leq 11$ Sig.: ***P $<0.001 ; * * 0.001 \leq \mathrm{P}<0.01 ; * 0.01 \leq \mathrm{P}<0.05$. 
PhD Dissertation Chapter 5

\section{Executive Summary}

Prepared by Rini Vohra

Submitted to Committee Members

Dr. Suresh Madhavan

Dr. Usha Sambamoorthi

Dr. Nilanjana Dwibedi

Dr. Claire StPeter

Dr. Susannah Poe 


\section{PhD Dissertation Chapter 5}

Table of Contents

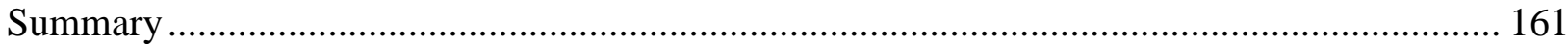

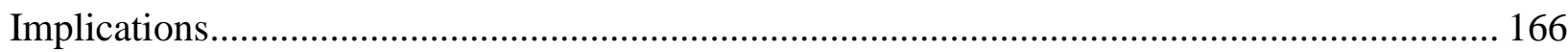

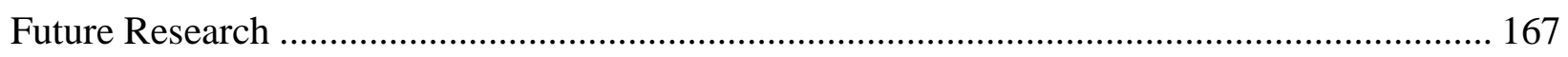

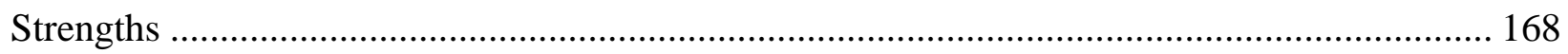

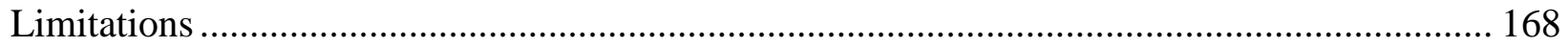

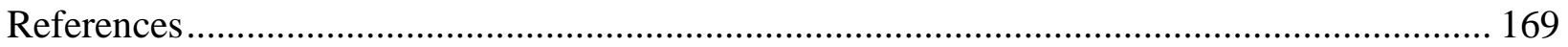




\section{PhD Dissertation Chapter 5}

\section{Summary}

In the past decade, awareness about autism spectrum disorders (ASD) has increased

(Autism Speaks, 2015) and more efforts are being put towards providing quality care to individuals affected by this condition. Even though vast number of studies have been conducted on health services utilization, health economics, and outcomes about children with ASD, there is extant literature on healthcare utilization and expenditures about adults with ASD. In addition, most existing studies on adults with ASD are restricted to small sample sizes, clinical or specialty settings which doesn't allow generalizability. The current dissertation research aimed to examine the burden of healthcare needs, healthcare utilization, and expenditures among adults with ASD as compared to a matched sample of adults without ASD. Considering the increasing prevalence of ASD in children and as large numbers of children with ASD transition to adults (Brugha et al., 2011; Developmental Disabilities Monitoring Network Surveillance Year 2010 Principal Investigators \& Centers for Disease Control and Prevention (CDC), 2014), our study is timely and critical for understanding the scope and nature of healthcare utilization and costs related to adults with ASD. In general, all three studies conducted as part of this research highlighted that adults with ASD are an at-risk population with high healthcare needs which transforms into substantial use of healthcare services and expenditures.

Our first study examined the prevalence of psychiatric, non-psychiatric comorbidities, other health conditions, healthcare utilization, and expenditures among adults with ASD as compared to adults without ASD enrolled in a fee for service Medicaid programs from 20002008. The study findings included: 1) Psychiatric comorbidities such as depression, anxiety, schizophrenia, and attention deficit disorders are higher among adults with ASD as compared to adults without ASD, an observation similar to children with ASD, which has been shown in few small sample studies (Lugnegard et al., 2011a; Lunsky et al., 2009; Unenge Hallerback et al., 


\section{PhD Dissertation Chapter 5}

2012); 2) Adults with ASD have lower yet modestly similar rates of non-psychiatric comorbidities as adults without ASD, except for gastrointestinal conditions which were significantly greater in the ASD group; 3) Adults with ASD also exhibit a significantly higher prevalence of other co-occurring conditions such as epilepsy, thyroid disease, lipid metabolism disorders, genito-urinary disorders, skin disorders, and infections as compared to adults without ASD, similar to findings of recent study by (Croen et al., 2015); 4) The burden of healthcare use such as average number of outpatient visits and prescription drug claims per year for adults with ASD were significantly greater as compared to adults without ASD; 5) Average number of emergency room visits per year were not significantly different and hospitalizations per year were lower among adults with and without ASD; 6) Adults with ASD have a higher total and specific expenditures for outpatient office visits, emergency room visits, and prescription drugs; and 7) The presence of a psychiatric and/or a non-psychiatric comorbidity may not contribute highly to the differences in healthcare utilization and expenditures between an age, gender, and race matched cohort of adults with and without ASD, but have a significant contribution to higher healthcare utilization and expenditures among adults with ASD.

Our second study examined the trends and rates of all and specific emergency department (ED) visits among adults with and without ASD. The main study findings were: 1) The number of adults with ASD using ED over time increased as shown in the 6 year trend; 3) Public payers including Medicaid/Medicare account for $80 \%$ of insurance coverage for ED use among adults with ASD; 3) For one-third of adults with ASD using the ED resulted in an inpatient hospital admission as compared to one-tenth of adults without ASD; 4) Both psychiatric and nonpsychiatric ED visits are more common among adults with ASD as compared to adults without ASD; 5) Overall, any injury visits were much less prevalent in adults with ASD as compared to 


\section{PhD Dissertation Chapter 5}

adults without ASD; 6) The most commonly associated reasons for ED visits among adults with ASD included: epilepsy, falls, schizophrenia, respiratory disorders, bipolar disorders, and depression; 7) A significantly greater proportion of adults with ASD reported self-harm, poisoning, and suicidal ideation during the ED visits as compared to adults without ASD; 8) Adults with ASD had 2.3 times the mean total ED costs for adults without ASD; and 9) The mean ED costs for psychiatric, non-psychiatric, as well as an injury visit was significantly higher for adults with ASD as compared to adults without ASD.

The third study of this three part research assessed the medication burden among adults with and without ASD. This study focused on examining the extent of prescription drug use and polypharmacy use among adults with and without ASD and the findings included: 1) Almost 95\% of adults with ASD had at least one prescription drug claim with a mean of 48 unique drug claims per year; 2) Psychotropic drug use among adults with ASD was extremely high (85\%), with the highest use of antipsychotics, followed by anticonvulsants, antidepressants, and anxiolytics/sedatives/hypnotics; 3) Use of prescription drug classes among adults with ASD were not restricted to psychiatric needs and a high proportion of adults with ASD also had drug claims for respiratory agents, immunologics, cardiovascular agents, hormone modifiers/steroids, statins/antihyperlipidemics, dermatologic agents, alternative medicines/supplements, gastrointestinal agents, and antiparkinson agents; 4) The mean number of prescription drug claims for such drug classes were higher among adults with ASD as compared to adults without ASD; 5) Rates of general (48\%) and psychotropic polypharmacy (19\%) among adults with ASD were high and they were more likely to use at least six or more prescription drug classes in a year and 3 or more psychotropic drug classes in a 90 day period as compared to adults with ASD; 6) From 2000 to 2008, the proportion of adults with ASD who used general polypharmacy showed 


\section{PhD Dissertation Chapter 5}

a sustained and significant rising trend; 7) Female adults with ASD were found to be more likely to have general polypharmacy as compared to male adults with ASD; 8) Number of comorbid conditions was the most critical and significant determinant of general and psychotropic polypharmacy in a specified period of time.

There are several themes that emerge from the findings of our three part research:

1. Unlike most previous studies which have reported high prevalence of psychiatric comorbidities among individuals with ASD, our study showed that adults with ASD have similar rates of non-psychiatric needs such as gastrointestinal disorders ,cardiovascular disorders, diabetes, lipid disorders, respiratory disorders, and many others as compared to adults without ASD. This finding was consistent in all three studies where adults with ASD showed high prevalence of these comorbidities, visited ED for such reasons, and also showed a higher prescription drug use for similar drug classes as compared to adults without ASD. A recent study by (Croen et al., 2015) indicated a similar pattern where adults with ASD were not only afflicted by psychiatric comorbid conditions but also had a high occurrence of other health conditions.

2. Injurious behaviors have long been reported among individuals with ASD (Adler et al., 2014; Matson \& Rivet, 2008), but very few studies have examined the actual implication of such behaviors. Our second study on ED use among adults with ASD showed that adults with ASD have a high degree of self-harm behaviors and suicidal ideation which leads to ED visits and possibly higher hospitalization rates.

3. Healthcare services use and costs among children with ASD have been established to be extremely high to the society as well as individuals with ASD (Croen et al., 2006; Lokhandwala et al., 2012; Wang \& Leslie, 2010a; Wang et al., 2012). However, there is 


\section{PhD Dissertation Chapter 5}

no current literature about the healthcare utilization and costs among adults with and without ASD. Our three part research is the first of its kind to report a high healthcare utilization and expenditures in a large sample of adults with ASD. The total mean Medicaid expenditures for adults with ASD were 1.6 times higher than adults without ASD. There have been previous studies that have shown increasing Medicaid expenditures for individuals with ASD (Mandell et al., 2006; Mandell, 2008a; Rosenberg et al., 2010a; Semansky et al., 2011a). With the increasing concern of rising Medicaid expenditures, our study provides substantial evidence about the overall and specific cost burden on Medicaid of providing healthcare services to adults with ASD.

4. Multiple medication use or polypharmacy is a critical issue among individuals with ASD due to lack of gold standard treatments and use of off-label prescription drugs (Dalsgaard et al., 2013; Frazier et al., 2011a; Warren et al., April 2011; Zito et al., 2008). Our study on medication burden among adults with ASD showed that there might not only be multiple classes of drugs used in a year, which many a time is a necessity, but psychotropic medication use among adults with ASD is very high which is of concern, since other than risperidone and aripiprazole (new generation antipsychotics), most of the psychotropic drugs are used off-label. This was evident in our study when individuals with ASD but no psychiatric or non-psychiatric comorbidity used three or more psychotropic drugs concurrently. Other than off-label use, side effects of psychotropic drugs such as risperidone which can cause serious adverse events is also a concern for adults with ASD. The effectiveness of these drugs have also received only medium to low level rating of evidence (Warren et al., April 2011). This highlights a great need for appropriate pharmacological guidelines for the treatment of adults with ASD. 


\section{PhD Dissertation Chapter 5}

\section{Implications}

Adults with ASD have high healthcare needs attributed to their core ASD symptoms as well as high prevalence of comorbid conditions. The implications of such needs are well reflected in our study findings where adults with ASD were shown to have higher healthcare utilization and expenditures as compared to adults without ASD. As noted in many previous studies (Heidgerken et al., 2005; Imran et al., 2011; Liptak et al., 2006; Miller, 2015; Piven et al., 2011), ASD knowledge and training among physicians and other specialty care providers have been reported to be low, and there is a lack in self-reported confidence among providers in being able to correctly identify ASD, understand their symptoms and pharmacological needs, and provide quality care that improves disease prognosis and reduce morbidity. In a scenario where there is no standard cure and two approved medications have only medium to low level effectiveness (Warren et al., April 2011), greater focus is needed to provide easy access to early behavioral intervention services, which have been shown to significantly improve prognosis of this condition (Autism Speaks, 2013; Bailey et al., 2004; Reichow, 2012). In addition, further studies are needed to address the poor rating of patient provider communication and greater unmet healthcare needs that have been reported by adults with ASD in a few studies (Magiati et al., 2014; Nicolaidis et al., 2013).

Medicaid coverage of healthcare services for adults with ASD is substantial and state programs bear the high costs of such services. Care should be directed towards managing both psychiatric and non-psychiatric needs of adults with ASD in the primary as well as specialty care settings. Future interventions must focus on better ASD training and education of healthcare providers as well as guidelines for treatment that assure quality and well-coordinated care for adults with ASD. (Miller, 2015) examined the extent of ASD knowledge among nurses working in an ED and found that more than half of ED nurses surveyed reported having accurate 


\section{PhD Dissertation Chapter 5}

knowledge, correctly identified causes of visit and comorbidities, and chose appropriate interventions for ASD cases. However, nurses still reported having limited knowledge and resources available to them and felt a need for ASD education early on in their training. This perceived need to overcome gaps in ASD related care among nurses may also be true for physicians and other healthcare providers. Future studies should examine the association of quality and consistency of care received by adults with ASD in the primary care setting with a patient's subsequent ED use, frequency of ED use, hospital admissions, and healthcare costs.

\section{Future Research}

The area of health outcomes for adults with ASD requires further investiagtion that will help establish the varying healthcare needs of this population and their health related quality of life. Currently, there are no epidemiological studies on prevalence of ASD in adults in the US, leaving little information on the exact burden of this condition in the older population. In addition, bigger clinical trials and more advanced research is needed to develop interventions that work on addressing the ASD core symptoms and improve daily functioning and productivity. As a follow up to our findings on psychotropic drug use among adults with ASD, future studies should examine the long term association of psychotropic drug use among adults with ASD on development of new conditions such as obesity, diabetes, and psychiatric conditions which are caused by high use of antipsychotics. More studies are needed to identify the employment opportunities for adults with ASD and state and federal policies that support such plans. There is also very little information on the distribution of patients with ASD across rehabilitation hospitals, residential facilities and intermediate care facilities that undermine their reported healthcare utilization, overall expenditures, and lifetime costs when analyzing a single data source such as state Medicaid database. 


\section{PhD Dissertation Chapter 5}

\section{Strengths}

Our three part research have provided very useful insights on healthcare needs, utilization, and expenditures among adults with ASD. The current dissertation study utilized secondary databases such as the Nationwide Emergency Database Sample (NEDS) by Agency of Healthcare and Research Quality (AHRQ) and three-state Medicaid databases, both of which have significant policy implications. Our study not only provided an exploratory analysis of the prevalence of health conditions among adults with ASD, but also tested the association between comorbidities and healthcare utilization and expenditures. The three studies have filled some of the gaps in the literature and provide information about the healthcare needs of adults with ASD.

\section{Limitations}

The current research work did not provide a patient reported needs, and thus may not include needs of home based care, daily functioning, and access to services other than healthcare. In addition, we did not include patients who were using home and community based waivers, were part of residential facilities, and/or intermediate care facilities, who may be much sicker and have worse outcomes than adults with ASD who are able to use Medicaid covered services through caretakers or on their own. This research was primarily cross sectional and we could not assess the longitudinal healthcare utilization and expenditures. The current research also did not assess costs including indirect costs and costs due to lost productivity which contribute highly to the substantial lifetime costs of individuals with ASD. 


\section{PhD Dissertation Chapter 5}

\section{References}

Adler, B. A., Wink, L. K., Early, M., Shaffer, R., Minshawi, N., McDougle, C. J., \& Erickson, C. A. (2014). Drugrefractory aggression, self-injurious behavior, and severe tantrums in autism spectrum disorders: A chart review study. Autism: The International Journal of Research and Practice, doi:1362361314524641 [pii]

Autism Speaks. (2013). High-quality early intervention for autism more than pays for itself. Retrieved from http://www.autismspeaks.org/science/science-news/high-quality-early-intervention-autism-more-pays-itself

Autism Speaks. (2015). 10 year timeline. Retrieved from https://www.autismspeaks.org/10year/timeline

Bailey, D. B.,Jr, Hebbeler, K., Scarborough, A., Spiker, D., \& Mallik, S. (2004). First experiences with early intervention: A national perspective. Pediatrics, 113(4), 887-896.

Brugha, T. S., McManus, S., Bankart, J., Scott, F., Purdon, S., Smith, J., . . Meltzer, H. (2011). Epidemiology of autism spectrum disorders in adults in the community in england. Archives of General Psychiatry, 68(5), 459465. doi:10.1001/archgenpsychiatry.2011.38; 10.1001/archgenpsychiatry.2011.38

Croen, L. A., Najjar, D. V., Ray, G. T., Lotspeich, L., \& Bernal, P. (2006). A comparison of health care utilization and costs of children with and without autism spectrum disorders in a large group-model health plan. Pediatrics, 118(4), e1203-11. doi:118/4/e1203 [pii]

Croen, L. A., Zerbo, O., Qian, Y., Massolo, M. L., Rich, S., Sidney, S., \& Kripke, C. (2015). The health status of adults on the autism spectrum. Autism: The International Journal of Research and Practice, doi:1362361315577517 [pii]

Dalsgaard, S., Nielsen, H. S., \& Simonsen, M. (2013). Five-fold increase in national prevalence rates of attentiondeficit/hyperactivity disorder medications for children and adolescents with autism spectrum disorder, attention-deficit/hyperactivity disorder, and other psychiatric disorders: A Danish register-based study. Journal of Child and Adolescent Psychopharmacology, 23(7), 432-439. doi:10.1089/cap.2012.0111 [doi]

Developmental Disabilities Monitoring Network Surveillance Year 2010 Principal Investigators, \& Centers for Disease Control and Prevention (CDC). (2014). Prevalence of autism spectrum disorder among children aged 8 years - autism and developmental disabilities monitoring network, 11 sites, united states, 2010. Morbidity and Mortality Weekly Report. Surveillance Summaries (Washington, D.C.: 2002), 63(2), 1-21. doi:ss6302a1 [pii]

Frazier, T. W., Shattuck, P. T., Narendorf, S. C., Cooper, B. P., Wagner, M., \& Spitznagel, E. L. (2011). Prevalence and correlates of psychotropic medication use in adolescents with an autism spectrum disorder with and without caregiver-reported attention-deficit/hyperactivity disorder. Journal of Child and Adolescent Psychopharmacology, 21(6), 571-579. doi:10.1089/cap.2011.0057 [doi]

Heidgerken, A. D., Geffken, G., Modi, A., \& Frakey, L. (2005). A survey of autism knowledge in a health care setting. Journal of Autism and Developmental Disorders, 35(3), 323-330.

Imran, N., Chaudry, M. R., Azeem, M. W., Bhatti, M. R., Choudhary, Z. I., \& Cheema, M. A. (2011). A survey of autism knowledge and attitudes among the healthcare professionals in lahore, pakistan. BMC Pediatrics, 11 , 107-2431-11-107. doi:10.1186/1471-2431-11-107 [doi]

Liptak, G. S., Orlando, M., Yingling, J. T., Theurer-Kaufman, K. L., Malay, D. P., Tompkins, L. A., \& Flynn, J. R. (2006). Satisfaction with primary health care received by families of children with developmental disabilities. Journal of Pediatric Health Care: Official Publication of National Association of Pediatric Nurse Associates \& Practitioners, 20(4), 245-252. doi:10.1016/j.pedhc.2005.12.008 


\section{PhD Dissertation Chapter 5}

Lokhandwala, T., Khanna, R., \& West-Strum, D. (2012). Hospitalization burden among individuals with autism. Journal of Autism and Developmental Disorders, 42(1), 95-104. doi:10.1007/s10803-011-1217-x [doi]

Lugnegard, T., Hallerback, M. U., \& Gillberg, C. (2011). Psychiatric comorbidity in young adults with a clinical diagnosis of Asperger syndrome. Research in Developmental Disabilities, 32(5), 1910-1917. doi:10.1016/j.ridd.2011.03.025 [doi]

Lunsky, Y., Gracey, C., \& Bradley, E. (2009). Adults with autism spectrum disorders using psychiatric hospitals in Ontario: Clinical profile and service needs. Research in Autism Spectrum Disorders, 3(4), 1006-1013.

Magiati, I., Tay, X. W., \& Howlin, P. (2014). Cognitive, language, social and behavioral outcomes in adults with autism spectrum disorders: A systematic review of longitudinal follow-up studies in adulthood. Clinical Psychology Review, 34(1), 73-86. doi:10.1016/j.cpr.2013.11.002 [doi]

Mandell, D. S. (2008). Psychiatric hospitalization among children with autism spectrum disorders. Journal of Autism and Developmental Disorders, 38(6), 1059-1065. doi:10.1007/s10803-007-0481-2 [doi]

Mandell, D. S., Cao, J., Ittenbach, R., \& Pinto-Martin, J. (2006). Medicaid expenditures for children with autistic spectrum disorders: 1994 to 1999. Journal of Autism and Developmental Disorders, 36(4), 475-485. doi:10.1007/s10803-006-0088-z

Matson, J. L., \& Rivet, T. T. (2008). Characteristics of challenging behaviors in adults with autistic disorder, PDDNOS, and intellectual disability. Journal of Intellectual \& Developmental Disability, 33(4), 323-329. doi:10.1080/13668250802492600; 10.1080/13668250802492600

Miller, S. M. (2015). Nurses caring for adults with autism in an emergency department: A survey of knowledge. Doctoral Projects, (7)

National Conference of State Legislatures. (2014). Recent medicaid prescription drug laws and strategies. Retrieved from http://www.ncsl.org/research/health/medicaid-pharmaceutical-laws-and-policies.aspx

Nicolaidis, C., Raymaker, D., McDonald, K., Dern, S., Boisclair, W. C., Ashkenazy, E., \& Baggs, A. (2013). Comparison of healthcare experiences in autistic and non-autistic adults: A cross-sectional online survey facilitated by an academic-community partnership. Journal of General Internal Medicine, 28(6), 761-769. doi:10.1007/s11606-012-2262-7 [doi]

Piven, J., Rabins, P., \& Autism-in-Older Adults Working Group. (2011). Autism spectrum disorders in older adults: Toward defining a research agenda. Journal of the American Geriatrics Society, 59(11), 2151-2155. doi:10.1111/j.1532-5415.2011.03632.x [doi]

Reichow, B. (2012). Overview of meta-analyses on early intensive behavioral intervention for young children with autism spectrum disorders. Journal of Autism and Developmental Disorders, 42(4), 512-520. doi:10.1007/s10803-011-1218-9; 10.1007/s10803-011-1218-9

Rosenberg, R. E., Mandell, D. S., Farmer, J. E., Law, J. K., Marvin, A. R., \& Law, P. A. (2010). Psychotropic medication use among children with autism spectrum disorders enrolled in a national registry, 2007-2008. Journal of Autism and Developmental Disorders, 40(3), 342-351. doi:10.1007/s10803-009-0878-1; 10.1007/s10803-009-0878-1

Semansky, R. M., Xie, M., \& Mandell, D. S. (2011). Medicaid's increasing role in treating youths with autism spectrum disorders. Psychiatric Services (Washington, D.C.), 62(6), 588. doi:10.1176/appi.ps.62.6.588; 10.1176/appi.ps.62.6.588 


\section{PhD Dissertation Chapter 5}

Unenge Hallerback, M., Lugnegard, T., \& Gillberg, C. (2012). Is autism spectrum disorder common in schizophrenia? Psychiatry Research, 198(1), 12-17. doi:10.1016/j.psychres.2012.01.016; 10.1016/j.psychres.2012.01.016

Wang, L., \& Leslie, D. L. (2010). Health care expenditures for children with autism spectrum disorders in medicaid. Journal of the American Academy of Child and Adolescent Psychiatry, 49(11), 1165-1171. doi:10.1016/j.jaac.2010.08.003; 10.1016/j.jaac.2010.08.003

Wang, L., Mandell, D. S., Lawer, L., Cidav, Z., \& Leslie, D. L. (2012). Healthcare service use and costs for autism spectrum disorder: A comparison between medicaid and private insurance. Journal of Autism and Developmental Disorders, doi:10.1007/s10803-012-1649-y

Warren, Z., Veenstra-VanderWeele, J., Stone, W., Bruzek, J. L., Nahmias, A. S., Foss-Feig, J. H., . . McPheeters, M. L. (April 2011). Therapies for children with autism spectrum disorders.. (Comparative Effectiveness Reviews no. 26 No. 11-EHC029-EF). Rockville (MD): Agency for Healthcare Research and Quality. doi:http://effectivehealthcare.ahrq.gov/ehc/products/106/651/Autism_Disorder_exec-summ.pdf

Zito, J. M., Derivan, A. T., Kratochvil, C. J., Safer, D. J., Fegert, J. M., \& Greenhill, L. L. (2008). Off-label psychopharmacologic prescribing for children: History supports close clinical monitoring. Child and Adolescent Psychiatry and Mental Health, 2(1), 24. doi:10.1186/1753-2000-2-24 [doi] 